\title{
EFFECTIVE HYDRAULIC CONDUCTIVITY OF BOUNDED, STRONGLY HETEROGENEOUS \\ POROUS MEDIA
}

\author{
by \\ Evangelos Konstantinos Paleologos
}

\author{
A Dissertation Submitted to the Faculty of the \\ DEPARTMENT OF HYDROLOGY AND WATER RESOURCES \\ In Partial Fulfillment of the Requirements \\ For the Degree of \\ DOCTOR OF PHILOSOPHY \\ WITH A MAJOR IN HYDROLOGY \\ In the Graduate College \\ THE UNIVERSITY OF ARIZONA
}




\title{
EFFECTIVE HYDRAULIC CONDUCTIVITY OF \\ BOUNDED, STRONGLY HETEROGENEOUS
}

POROUS MEDIA

\author{
Evangelos Konstantinos Paleologos, Ph.D. \\ The University of Arizona, 1994
}

Director: Dr. Shlomo P. Neuman, Regents' Professor

This dissertation develops analytical expressions for the effective hydraulic conductivity $K_{e}$ of a three-dimensional porous medium bounded by two parallel planes of infinite extent separated by a distance $2 a$. Head varies randomly along each boundary about a uniform mean value. The log hydraulic conductivity $Y$ forms a homogeneous, statistically anisotropic random field having a variance $\sigma_{Y}^{2}$ and principal integral scales $\lambda_{1}, \lambda_{2}, \lambda_{3}$. Flow is uniform in the mean parallel to the principal coordinate $x_{1}$. A solution is first derived for mildly nonuniform media with $\sigma_{Y}^{2} \ll 1$ via an approximate form of the 1993 residual flux theory by Neuman and Orr. It is then extended to strongly nonuniform media with arbitrarily large $\sigma_{Y}^{2}$ by invoking the Landau-Lifshitz conjecture as $K_{e}=K_{G} \exp \left\{\sigma_{Y}^{2}[1 / 2-(\mathcal{D}+\mathcal{S})]\right\}$. Here, $K_{G}$ is the geometric mean of hydraulic conductivities and $\mathcal{D}$ and $\mathcal{S}$ are domain and surface integrals, respectively. Based on a rigorous limiting analysis we show that when the length scale ratio $\rho=a / \lambda_{1} \rightarrow 0, K_{e}$ is equal to the arithmetic mean hydraulic conductivity $K_{A}$. This supports the theoretical finding of Neuman and Orr and the numerical result by Desbarats. When $\rho \rightarrow \infty$ we obtain expressions for $K_{e}$ that 
have been previously derived in the stochastic literature for infinite flow domains. For strongly anisotropic media with integral scale ratios $\varepsilon_{2}=\lambda_{2} / \lambda_{1}$ and $\varepsilon_{3}=\lambda_{3} / \lambda_{1}$ equal to each other and tending to zero or infinity $\left(\lambda_{1} \neq 0\right)$ we obtain the closed form solution $K_{e}=K_{G} \exp \left\{\sigma_{Y}^{2}[\exp (-\rho)-0.5]\right\}$. The latter reduces to $K_{A}$ when $\rho \rightarrow 0$ and tends to the harmonic mean $K_{H}$ as $\rho \rightarrow \infty$. One can think of the case $\varepsilon_{2}=\varepsilon_{3}=0$ as mean flow along parallel channels having mutually uncorrelated hydraulic conductivities, and of the case $\varepsilon_{2}=\varepsilon_{3} \rightarrow \infty$ as mean flow normal to layers having uniform hydraulic conductivities. For statistically isotropic media we show numerically that $K_{e}$ equals $K_{A}$ when $\rho=0.01$; when $\rho \geq 4, K_{e}=K_{G} \exp \left(\sigma_{Y}^{2} / 6\right)$ the three-dimensional infinite domain solution. Our results support the analytical finding of Rubin and Dagan, and predict and explain all related bounded domain numerical results. Finally, contrary to Dagan's assertion, we show that for small $\rho$ boundary effects are extremely important; the absolute value of the surface integral $\mathcal{S}$ equals the value of the domain integral $\mathcal{D}$. 


\title{
EFFECTIVE HYDRAULIC CONDUCTIVITY OF BOUNDED, STRONGLY HETEROGENEOUS \\ POROUS MEDIA
}

\author{
by \\ Evangelos Konstantinos Paleologos
}

A Dissertation Submitted to the Faculty of the

DEPARTMENT OF HYDROLOGY AND WATER RESOURCES

In Partial Fulfillment of the Requirements

For the Degree of

DOCTOR OF PHILOSOPHY

WITH A MAJOR IN HYDROLOGY

In the Graduate College

THE UNIVERSITY OF ARIZONA 
THE UNIVERSITY OF ARIZONA

GRADUATE COLLEGE

As members of the Final Examination Committee, we certify that we have

read the dissertation prepared by Evangelos Konstantinos Paleologos

entitled Effective hydraulic conductivity of bounded, strongly

heterogeneous porous media

and recommend that it be accepted as fulfilling the dissertation

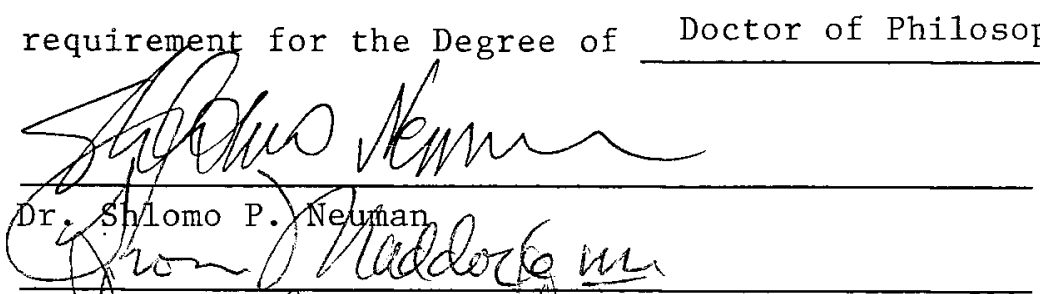

Dr. Thomas Maddolck III

Nolletel

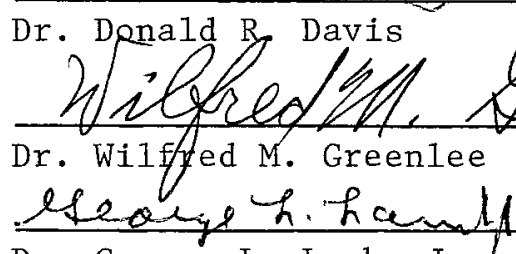
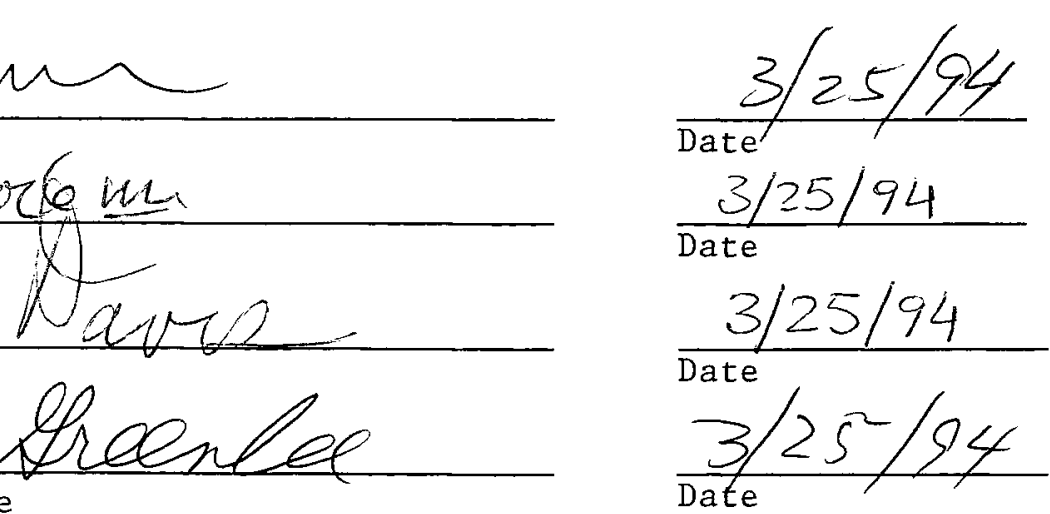

Dr. George L. Lamb, Jr.

Marih 25,1994

Final approval and acceptance of this dissertation is contingent upon the candidate's submission of the final copy of the dissertation to the Graduate College.

I hereby certify that I have read this dissertation prepared under my direction and recommend that it be accepted as fulfilling the dissertation requirement.
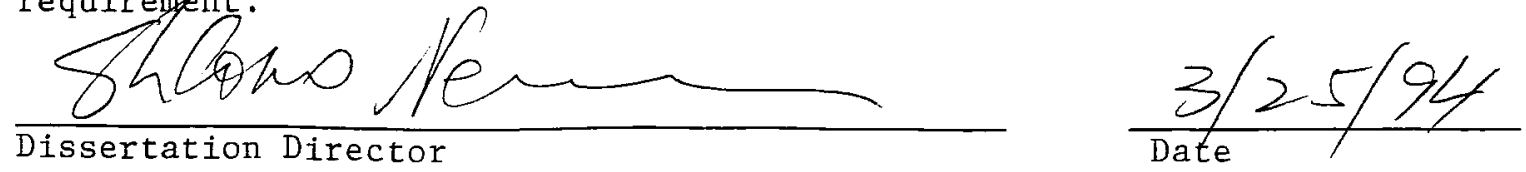

Dr. Shlomo P. Neuman 


\section{STATEMENT BY AUTHOR}

This dissertation has been submitted in partial fulfillment of requirements for an advanced degree at The University of Arizona and is deposited in the University Library to be made available to borrowers under rules of the Library.

Brief quotations from this dissertation are allowable without special permission, provided that accurate acknowledgement of source is made. Requests for permission for extended quotation from or reproduction of this manuscript in whole or in part may be granted by the head of the major department or the Dean of the Graduate College when in his or her judgement the proposed use of the material is in the interests of scholarship. In all other instances, however, permission must be obtained from the author.

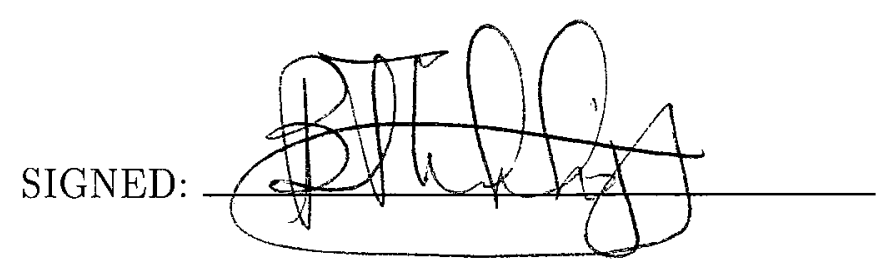

\section{APPROVAL BY DISSERTATION DIRECTOR}

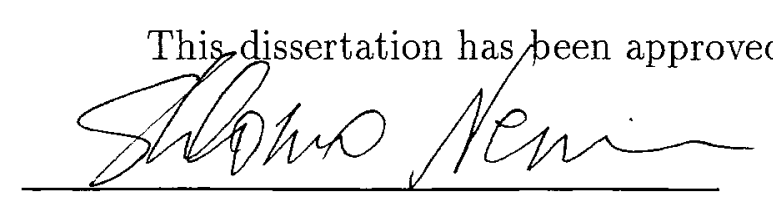

Shlomo P. Neuman on the date shown below:

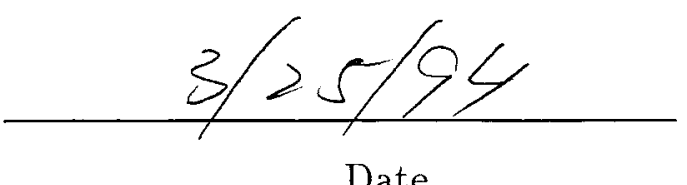

Date

Professor of Hydrology 
In memory of my father, Konstantinos Paleologos,

whose scientific excellence and encouragement provided

the inspiration for this dissertation. 


\section{ACKNOWLEDGEMENTS}

This dissertation was supported jointly by the U.S. Nuclear Regulatory Commission under contract NRC-04-90-51, and the U.S. Geological Survey under Water Resources Research Grant 14-08-0001-G2092. I am grateful to Dr. Shlomo P. Neuman, the principal investigator of these projects, for providing me with a research associateship during my graduate studies.

I would further like to thank my advisor Dr. Shlomo P. Neuman for his guidance, constructive criticism, and encouragement during the development of this dissertation. Above all, with his standards of excellence in scientific research he has truly been a teacher to me, by guiding, demanding, and inspiring me to become a better scientist.

I would also like to thank Dr. Thomas Balsa, Dr. Wilfred Greenlee, and Dr. Thomas Maddock III for their valuable assistance. I would especially like to thank my friend George Sochos for the mathematical insight and suggestions at different stages of the development of this dissertation.

I would like to thank my mother Aikaterini, my sister Angela, and my cousin Dr. Vlasios K. Koumousis for their love and support. At last but not least, I would like to thank my dear wife Cleo for the sacrifices she had to endure during my studies, her love and patience, and for giving me our lovely daughter Katrina. 


\section{TABLE OF CONTENTS}

1 INTRODUCTION 14

1.1 Introduction and Scope . . . . . . . . . . . . . . 14

1.2 Dissertation Preview . . . . . . . . . . . . . . . . . . . 19

2 LITERATURE REVIEW 21

2.1 Infinite Domain Solutions . . . . . . . . . . . . . . . . 21

2.1.1 Small Perturbation and Spectral Approach . . . . . . . . . 22

2.1.2 Asymptotic Expansion and Green's Function Approach . . . . 31

2.1.3 Method of Embedding Matrix . . . . . . . . . . . 35

2.1.4 Method of Moments for Periodic Media . . . . . . . . . . . . 36

2.1.5 Ad Hoc Power-Averaging Method . . . . . . . . . . . . . 42

2.2 Bounded Domain Solutions . . . . . . . . . . . . . . . . 45

2.2.1 Small Perturbation and Green's Function Approach . . . . . . 45

2.2.2 Numerical Studies via Monte Carlo Simulation . . . . . . . . . 46

3 RESIDUAL FLUX THEORY 52

3.1 Basic Equation for the Residual Flux . . . . . . . . . . . . 52

3.2 Comparison of Residual Flux Theory to other Theories . . . . . . . 57

3.2.1 Non-Stationarity of Head Fluctuations in Bounded Domains . 58

3.2.2 Asymptotic Expansion and Green's Function Approach . . . . 60

3.3 Effective Conductivity for Infinite Domains . . . . . . . . . . . . . 61 
4 EFFECTIVE CONDUCTIVITY FOR 3-D BOUNDED DOMAINS 69

4.1 Basic Equation for the Effective Hydraulic Conductivity . . . . . . 69

4.2 Two-Dimensional FT of the Covariance Function . . . . . . . . . 76

4.3 Two-Dimensional FT of the Derivatives of $G_{F} \ldots \ldots . . . . . .79$

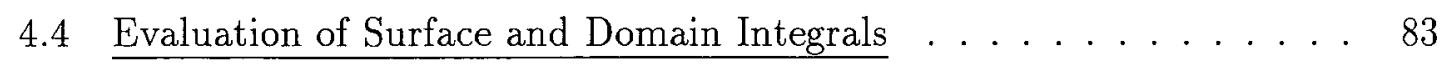

4.4.1 General Expression for the Surface Integral . . . . . . . . . 85

4.4.2 General Expression for the Domain Integral . . . . . . . . . 90

4.5 General Expression for Effective Conductivity . . . . . . . . . . . . 92

5 ASYMPTOTIC ANALYSIS AND NUMERICAL RESULTS 94

5.1 Boundaries Come Arbitrarily Close Together . . . . . . . . . . . . 94

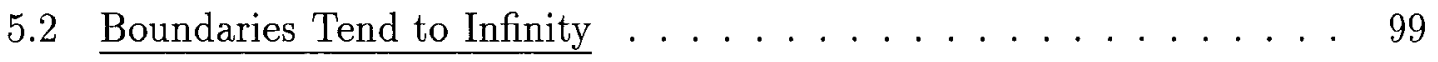

5.3 Analytical Results for Anisotropic Media . . . . . . . . . . . . . . . 104

5.4 Numerical Results for Isotropic Media . . . . . . . . . . . . . . . . 107

6 CONCLUSIONS $\quad 130$

APPENDICES:

A DERIVATION OF EXPRESSION FOR THE COVARIANCE OF THE HYDRAULIC CONDUCTIVITY

B DERIVATION OF PARSEVAL'S IDENTITIES

C FOURIER TRANSFORM OF MIXED DERIVATIVES OF 3- AND 2-DIMENSIONAL INFINITE GREEN'S FUNCTION 138

D EVALUATION OF THE LEGENDRE POLYNOMIAL $P_{1}(x) \quad 140$ 
E EVALUATION OF THE INTEGRAL $\mathcal{E}$

F DERIVATION OF EQUATION 3.35 144

G 3-DIMENSIONAL FOURIER TRANSFORM OF EXPONENTIAL $\begin{array}{ll}\text { COVARIANCE } & 148\end{array}$

H EVALUATION OF INTEGRAL TERM OF EQUATION F.10 151

I DERIVATION OF EQUATION F.17 153

J EFFECTIVE CONDUCTIVITY IN TWO-DIMENSIONAL INFI$\begin{array}{ll}\text { NITE DOMAINS } & \mathbf{1 5 5}\end{array}$

K NUMERICAL EVALUATION USING GAUSSIAN QUADRATURE

L DERIVATION OF EQUATION 4.43

M SOLUTION OF EQUATION 4.53

N ONE-DIMENSIONAL FT OF EQUATION 4.67

O DERIVATION OF THE SUM OF THE SERIES IN 4.66

P SOLUTION FOR $\chi_{1} \neq x_{1}$, EQUATION 4.65 


\section{LIST OF FIGURES}

2.1 Dykaar and Kitanidis [1992b]: Numerical evaluation of (2.67) for isotropic exponential and Gaussian input covariances. . . . . . . . . . 43

2.2 Dykaar and Kitanidis [1992b]: Comparison between (2.26), (2.27), (2.53), and numerical $K_{e} \ldots \ldots \ldots \ldots 4$

2.3 Fenton and Griffiths [1993]: Block conductivity in rectangular domain. 51

3.1 Comparison between $(2.26),(2.27)$ and numerical $K_{e} \ldots \ldots$. . . 68

4.1 The flow domain $\Omega \ldots \ldots \ldots \ldots$. . . . . . . . . . 70

4.2 Green's function's infinite series of images. . . . . . . . . . . . . . 73

4.3 Dirac delta sequence $\delta_{a}\left(\eta_{1}\right) \ldots \ldots \ldots$. . . . . . . . . . 87

5.1 Normalized $K_{e}$, equation 5.62, as a function of $\sigma_{Y}^{2}$ and $2 \rho . \ldots . . .106$

5.2 Surface and domain integrands, (4.110) and (4.113), $\rho=50$ and $j=0.116$

5.3 Surface integrand, (4.110), $\rho=4, j=0$, and $j=10 \ldots \ldots 117$

5.4 Domain integrand, $(4.113), \rho=4, j=0$ (top), and $j= \pm 2 \ldots \ldots$

5.5 Surface integrand, $(4.110), \rho=2, j=0$, and $j=10 \ldots \ldots$. . . . 119

5.6 Domain integrand, $(4.113), \rho=2, j=0$ (top), and $j= \pm 4 \ldots \ldots 120$

5.7 Surface integrand, $(4.110), \rho=1, j=0$, and $j=20 \ldots \ldots 121$

5.8 Domain integrand, $(4.113), \rho=1, j=0$ (top), and $j= \pm 4 \ldots \ldots 122$

5.9 Surface integrand, $(4.110), \rho=0.01, j=0$, and $j=100 . \ldots 123$

5.10 Domain integrand, (4.113), $\rho=0.01, j=0$ (top), and $j= \pm 100 . . .124$ 
5.11 Normalized $K_{e}$, equation 4.115 , as a function of $\sigma_{Y}^{2}$ for various $2 \rho \ldots 125$

5.12 Normalized $K_{e}$, equation 4.115, as a function of $2 \rho$ for various $\sigma_{Y}^{2} \ldots 126$

5.13 Normalized $K_{e}^{L}$, equation 4.114 , as a function of $\sigma_{Y}^{2}$ and $2 \rho . \ldots 127$

5.14 Domain integral, equation 4.113 , as a function of $2 \rho . \ldots 128$

5.15 Surface integral, equation 4.110 , as a function of $2 \rho . \ldots . . . . \quad 129$ 


\section{LIST OF TABLES}

5.1 Effective Hydraulic Conductivity in Bounded Domains. . . . . . . . . 110

$5.2 K_{e}$ values in bounded domains: method of moments and residual flux theory. . . . . . . . . . . . . . . . 115 


\begin{abstract}
This dissertation develops analytical expressions for the effective hydraulic conductivity $K_{e}$ of a three-dimensional porous medium bounded by two parallel planes of infinite extent separated by a distance $2 a$. Head varies randomly along each boundary about a uniform mean value. The log hydraulic conductivity $Y$ forms a homogeneous, statistically anisotropic random field having a variance $\sigma_{Y}^{2}$ and principal integral scales $\lambda_{1}, \lambda_{2}, \lambda_{3}$. Flow is uniform in the mean parallel to the principal coordinate $x_{1}$. A solution is first derived for mildly nonuniform media with $\sigma_{Y}^{2} \ll 1$ via an approximate form of the 1993 residual flux theory by Neuman and Orr. It is then extended to strongly nonuniform media with arbitrarily large $\sigma_{Y}^{2}$ by invoking the Landau-Lifshitz conjecture as $K_{e}=K_{G} \exp \left\{\sigma_{Y}^{2}[1 / 2-(\mathcal{D}+\mathcal{S})]\right\}$. Here, $K_{G}$ is the geometric mean of hydraulic conductivities and $\mathcal{D}$ and $\mathcal{S}$ are domain and surface integrals, respectively. Based on a rigorous limiting analysis we show that when the length scale ratio $\rho=a / \lambda_{1} \rightarrow 0, K_{e}$ is equal to the arithmetic mean hydraulic conductivity $K_{A}$. This supports the theoretical finding of Neuman and Orr and the numerical result by Desbarats. When $\rho \rightarrow \infty$ we obtain expressions for $K_{e}$ that have been previously derived in the stochastic literature for infinite flow domains. For strongly anisotropic media with integral scale ratios $\varepsilon_{2}=\lambda_{2} / \lambda_{1}$ and $\varepsilon_{3}=\lambda_{3} / \lambda_{1}$ equal to each other and tending to zero or infinity $\left(\lambda_{1} \neq 0\right)$ we obtain the closed form solution $K_{e}=K_{G} \exp \left\{\sigma_{Y}^{2}[\exp (-\rho)-0.5]\right\}$. The latter reduces to $K_{A}$ when $\rho \rightarrow 0$ and tends to the harmonic mean $K_{H}$ as $\rho \rightarrow \infty$. One can think of the case $\varepsilon_{2}=\varepsilon_{3}=0$ as mean flow along parallel channels having mutually uncorrelated hydraulic conductivities, and of the case $\varepsilon_{2}=\varepsilon_{3} \rightarrow \infty$ as mean flow normal to layers having uniform hydraulic conductivities. For statistically isotropic media we show
\end{abstract}


numerically that $K_{e}$ equals $K_{A}$ when $\rho=0.01 ;$ when $\rho \geq 4, K_{e}=K_{G} \exp \left(\sigma_{Y}^{2} / 6\right)$ the three-dimensional infinite domain solution. Our results support the analytical finding of Rubin and Dagan, and predict and explain all related bounded domain numerical results. Finally, contrary to Dagan's assertion, we show that for small $\rho$ boundary effects are extremely important; the absolute value of the surface integral $\mathcal{S}$ equals the value of the domain integral $\mathcal{D}$. 


\section{CHAPTER 1}

\section{INTRODUCTION}

\subsection{Introduction and Scope}

During the last two decades an increasing number of scientists in the field of subsurface hydrology have adopted a statistical (over a deterministic) framework in the study of flow and transport through porous media [Dagan, 1986]. Such a conceptual approach came about from the recognition of (i) the spatial variability of parameters and variables of interest [Freeze, 1975; Smith and Freeze, 1979a, b; Neuman, 1982; Hoeksema and Kitanidis, 1985; Dagan, 1986; Gelhar, 1986; Sudicky, 1986], (ii) the uncertainty arising from the incomplete characterization of this variability and/or measurement and interpretive errors [Delhomme, 1979; Clifton and Neuman, 1982; Dagan, 1982a; Gómez-Hernández and Gorelick, 1989; Desbarats and Dimitrakopoulos, 1990; Neuman and Orr, 1993], (iii) the uncertainty in boundary and/or initial conditions and source terms [Gelhar, 1986; Gómez-Hernández and Gorelick, 1989; Neuman and Orr, 1993], and (iv) the versatility of the statistical approach to

address relationships at different scales [Cushman, 1983b, 1984, 1986, 1987, 1993; Baveye and Sposito, 1984, 1985; Dagan, 1986; Neuman, 1990, 1991, 1994].

In this thesis, randomness is primarily associated with the hydraulic conductivity $K(\boldsymbol{x})$ of soils and rocks, found to vary erratically in space over several orders of magnitude [Bakr, 1976; Clifton and Neuman, 1982; Sudicky, 1986; Neuman and 
Depner, 1988; Holmes et al., 1990; Winberg, 1991]. In line with existing stochastic subsurface flow theories, $K(\boldsymbol{x})$ is depicted here as a random function [Matheron, 1971; Dagan, 1989], viewed locally as a scalar [Bakr et al., 1978; Gelhar and Axness, 1983a; Neuman and Depner, 1988; Dagan, 1989]. We address also uncertainty introduced from the scarcity of data on aquifer boundaries and source terms [Gelhar, 1986; Neuman and Orr, 1993], by treating the prescribed source, head and flux in a similar manner.

The most general description of steady state saturated flow, on a local scale $\omega$ which is small compared to the flow domain $\Omega$, is given by

$$
-\nabla \cdot \boldsymbol{q}(\boldsymbol{x})+f(\boldsymbol{x})=0 \quad \text { for } \boldsymbol{x} \in \Omega,
$$

subject to the boundary conditions

$$
\begin{array}{ll}
h(\boldsymbol{x})=H(\boldsymbol{x}) & \text { for } \boldsymbol{x} \in \Gamma_{D}, \\
-\boldsymbol{q}(\boldsymbol{x}) \cdot \boldsymbol{n}(\boldsymbol{x})=Q(\boldsymbol{x}) & \text { for } \boldsymbol{x} \in \Gamma_{N},
\end{array}
$$

and where, according to Darcy's law,

$$
\boldsymbol{q}(\boldsymbol{x})=-K(\boldsymbol{x}) \nabla h(\boldsymbol{x}) .
$$

Here the flux $\boldsymbol{q}(\boldsymbol{x}), K(\boldsymbol{x})$, the gradient of head $h(\boldsymbol{x})$, and the randomly prescribed source function $f(\boldsymbol{x})$ are defined on this volume $\omega$ (centered about the point $\boldsymbol{x}$ ) on which Darcy's law (1.4) is taken to apply. $H(\boldsymbol{x})$ and $Q(\boldsymbol{x})$ are randomly prescribed head and flux functions on the Dirichlet and Neumann boundary segments $\Gamma_{D}$ and $\Gamma_{N}$, respectively, and $\boldsymbol{n}(\boldsymbol{x})$ is a unit outward normal on the total boundary $\Gamma=\Gamma_{D} \cup \Gamma_{N}$. Equations (1.1)-(1.4) are the starting point of our analysis, with the stipulation of statistical independence for the prescribed head, flux and source term. 
The above, general, description of steady state saturated flow is usually reduced in the stochastic literature to

$$
-\nabla \cdot \boldsymbol{q}(\boldsymbol{x})=0 \quad ; \quad \boldsymbol{q}(\boldsymbol{x})=-K(\boldsymbol{x}) \nabla h(\boldsymbol{x}),
$$

subject to deterministic boundary conditions in (1.2) and (1.3). One typically prescribes $H$ deterministically, sometimes $Q$ (most often $Q=0$ ). Equation 1.5 is often analyzed in the form of

$$
\nabla^{2} h(\boldsymbol{x})+\nabla Y(\boldsymbol{x}) \cdot \nabla h(\boldsymbol{x})=0,
$$

where the random function $Y(\boldsymbol{x})=\ln K(\boldsymbol{x})$ is characterized by smaller fluctuations around its mean than the original $K$-field, thus making possible the use of small perturbations theory in the study of (1.6).

In order to solve, either analytically or numerically, the stochastic flow equation 1.6 for moments of head and/or flux, the joint probability density function of the random function $Y(\boldsymbol{x})$ needs to be specified over the entire flow field. The difficulty of such a task for real-life situations makes necessary the introduction of simplifying assumptions. The hypothesis of weak stationarity (or statistical homogeneity), usually invoked for the log hydraulic conductivity field, implies that the expected value of $Y$ is the same at all points, and that the autocovariance $C_{Y}(\boldsymbol{r})$ is a function of the separation vector $\boldsymbol{r}=\boldsymbol{x}_{i}-\boldsymbol{x}_{j}$ but not of the specific locations $\boldsymbol{x}_{i}$ and $\boldsymbol{x}_{j}$. When the spatial correlation is independent of direction, i.e. $C_{Y}$ is a function of $r=|\boldsymbol{r}|$, the medium is called statistically isotropic, otherwise it is anisotropic. Hydraulic conductivity data often seem to fit a lognormal distribution [Freeze, 1975; Delhomme, 1979; Neuman, 1982; Hoeksema and Kitanidis, 1985; Gelhar, 1986; Sudicky, 1986], though other distributions may also fit such data [Smith, 1981; Jones et al., 1985; 
Woodbury and Sudicky, 1991]. This has commonly been interpreted to mean that $Y(\boldsymbol{x})$ is a multivariate Gaussian field (which the former does not necessarily imply). This makes the analysis of (1.6) much easier, allowing one to make use of existing theory on the properties of Gaussian fields. The assumptions of weak stationarity and multivariate normality, together with a functional form for the autocovariance of $Y(\boldsymbol{x})$, provide a complete statistical description of spatial heterogeneity [Dagan, 1989, Chapters 1.2 and 3.2]. Underlying all of this is the concept of an ensemble of many, equally possible aquifers, the real aquifer being a single realization of this ensemble. Since in reality one always deals with a specific geologic formation rather than an ensemble of formations, the sample moments of measurable data $(Y, h)$ cannot be taken to represent ensemble moments without invoking the hypothesis of ergodicity. Ergodicity means that the probability law (or moments) governing a random function can be derived either from repeated sampling of an ensemble of statistically equivalent media at given points in space, or from samples collected at different points within a single medium (that is, from a single realization of the stochastic process) [Neuman, 1982; Papoulis, 1984; Priestley, 1989].

The development of expressions for the effective hydraulic conductivity $\boldsymbol{K}_{e}(\boldsymbol{x})$, which comprises the subject of our study, constitutes an area of important effort in the stochastic literature. The traditional concept of effective conductivity is limited to a relatively narrow class of flow problems [Neuman and Orr, 1993]. Where the concept applies, it allows replacing a random nonuniform medium by a fictitious "equivalent" uniform medium which preserves the original "average hydraulic behavior". In terms of ensemble moments, one can replace (1.5) by

$$
\nabla \cdot\langle q(\boldsymbol{x})\rangle=0 \quad ; \quad\langle q(\boldsymbol{x})\rangle=-\boldsymbol{K}_{e}(\boldsymbol{x}) \cdot \nabla\langle h(\boldsymbol{x})\rangle
$$


where $<\cdot>$ denotes ensemble mean. Although the first equation in (1.7) is merely the ensemble average of the continuity equation in (1.5), the second relationship does not follow directly from ensemble averaging of Darcy's law (see Chapter 3) and is not valid except under special circumstances [Dagan, 1989; Neuman and Orr, 1993].

The representation in (1.7) constitutes a definition of $\boldsymbol{K}_{e}$ as utilized in our study. It coincides with similar definitions by Matheron [1967], Freeze [1975], Gutjahr et al. [1978], Dagan [1979, 1981, 1982a, 1982b, 1986, 1989], Smith and Freeze [1979b], Gelhar and Axness [1981, 1983a], Gelhar [1986], Poley [1988], Naff [1991], and Neuman and Orr [1993]. In studies concerned with the problem of upscaling, i.e. defining block averaged conductivities from point variables, or relating measurements and properties on different scales, $\boldsymbol{K}_{e}^{i}$ is defined for each realization $i$ of the ensemble as the ratio between spatially averaged flux and head gradient [Desbarats 1987a, 1992a; Desbarats and Dimitrakopoulos, 1990; Rubin and Gómez-Hernández, 1990; Fenton and Griffiths, 1993]. The ensemble mean of these $\boldsymbol{K}_{e}^{i}$ corresponds to our definition of an effective hydraulic conductivity over the averaging volume.

Analytical expressions for $\boldsymbol{K}_{e}$ have been presented by numerous authors including Matheron [1967], Gutjahr et al. [1978], Dagan [1979, 1981, 1982a, 1982b, 1989], Gelhar and Axness [1981, 1983a], Gelhar [1986], Neuman and Depner [1988], Poley [1988], and Naff [1991]. The determination of $\boldsymbol{K}_{e}$ via Monte Carlo simulations under uniform mean flow has been pursued by Freeze [1975], Smith and Freeze [1979b], El-Kadi and Brutsaert [1985], Desbarats [1987a, 1987b, 1992a, 1992b], Gómez-Hernández and Gorelick [1989], Desbarats and Dimitrakopoulos [1990], Rubin and Gómez-Hernández [1990], Fenton and Griffiths [1993], and Neuman and Orr [1993]. 
The focus of all the previous analytical work has been on heterogeneous media of infinite extent. In this dissertation we offer, for the first time, analytical expressions for the effective conductivity of a bounded flow domain. These analytical solutions will be seen to be in excellent agreement with bounded domain results obtained independently by means of numerical methods. They also confirm a theoretical conclusion by Neuman and Orr [1993] regarding the behavior of $\boldsymbol{K}_{e}$ in the limit as $\Omega \rightarrow \omega$

\subsection{Dissertation Preview}

This dissertation is organized as follows. Chapter 2 reviews stochastic and deterministic theories by means of which analytical expressions for $\boldsymbol{K}_{e}$ in infinite flow domains have been derived in the past. We also present the numerical Monte Carlo studies of flow in bounded domains that are related to our topic, the results of which are later compared to our analytical expressions. Chapter 3 contains elements of the residual flux theory [Neuman and Orr, 1993] that this thesis relies on. The advantages of this approach over other existing theories, for flow in finite domains, are highlighted. The generality of the residual flux theory is shown by replicating some known results for domains of infinite extent. In Chapter 4, we develop expressions for the effective hydraulic conductivity in three-dimensional statistically anisotropic porous media which account for the presence of given mean head boundaries. Chapter 5 presents asymptotic analyses when these boundaries come arbitrarily close together and when they tend to infinity. For some anisotropic cases we obtain a simple closed form expression for the effective hydraulic conductivity. For isotropic media we show numerically that the infinite domain solution is a good approximation when the dis- 
tance between the two constant head planes exceeds 8 integral scales. Our results are shown here to be in excellent agreement with those from numerical Monte Carlo studies of flow in bounded domains. Finally, Chapter 6 presents the major conclusions from this study. 


\section{CHAPTER 2}

\section{LITERATURE REVIEW}

In the stochastic literature, analytical expressions for effective hydraulic conductivity have been reported mostly for steady state saturated groundwater flow under a uniform mean hydraulic gradient in an infinite, stationary, lognormal $K(\boldsymbol{x})$ field. In this chapter we review solutions for $\boldsymbol{K}_{e}$ for heterogeneous media subject to the above flow conditions in domains of both infinite and finite extent. Our discussion focuses on theory rather than results, with the aim of elucidating their assumptions and ranges of validity. For this, we sometimes include detailed derivations of the more significant solutions for $\boldsymbol{K}_{e}$ to expose the source of the relevant assumptions. Various solutions and intermediate expressions are compared to reveal similarities and differences between the underlying formalisms. This chapter also sets the stage for an in depth examination in Chapter 3 of the range of validity of existing theories in light of the residual flux theory developed by Neuman and Orr [1993] on which this dissertation relies.

\subsection{Infinite Domain Solutions}

Analytical solutions for $\boldsymbol{K}_{e}$ have been published only for infinite flow domains; bounded domains have been analyzed only numerically. We commence by reviewing some of these theories and results. 


\subsubsection{Small Perturbation and Spectral Approach}

The small perturbation and spectral approach [Bakr et al., 1978; Gutjahr et al., 1978; Gelhar and Axness, 1981, 1983a; Gelhar, 1986] has been historically the stochastic theory by means of which the majority of expressions for $\boldsymbol{K}_{e}$, for both statistically isotropic and anisotropic media, have been derived. The starting point is the following equation for steady state flow in a heterogeneous porous medium [Bakr et al., 1978, equation 14; Gelhar and Axness, 1983a, equation 11; Gelhar, 1986, equation $6]$,

$$
\frac{\partial}{\partial x_{i}}\left(K \frac{\partial h}{\partial x_{i}}\right)=0
$$

where the Einstein summation convention is utilized here and in the rest of this subsection. If $K \neq 0$ and we set $Y=\ln K$, then (2.1) can be rewritten as [Bakr at al., 1978, equation 15; Gelhar, 1986, equation 7]

$$
\frac{\partial^{2} h}{\partial x_{i}^{2}}+\frac{\partial Y}{\partial x_{i}} \frac{\partial h}{\partial x_{i}}=0
$$

Representing $h$ and $Y$ in terms of their mean values and fluctuations $h^{\prime}$ and $Y^{\prime}$ about these means according to [Bakr et al., 1978, equation 4; Gelhar and Axness, 1983a, equations 2 and 23; Gelhar, 1986, p. 137S]

$$
\begin{gathered}
h(\boldsymbol{x})=H(\boldsymbol{x})+h^{\prime}(\boldsymbol{x}) ; \quad H(\boldsymbol{x})=\langle h(\boldsymbol{x})\rangle, \quad\left\langle h^{\prime}(\boldsymbol{x})\right\rangle=0 \\
Y(\boldsymbol{x})=F(\boldsymbol{x})+Y^{\prime}(\boldsymbol{x}) ; \quad F(\boldsymbol{x})=\langle Y(\boldsymbol{x})\rangle, \quad\left\langle Y^{\prime}(\boldsymbol{x})\right\rangle=0,
\end{gathered}
$$

allows rewriting equation 2.2 as

$$
\frac{\partial^{2} H}{\partial x_{i}^{2}}+\frac{\partial^{2} h^{\prime}}{\partial x_{i}^{2}}+\frac{\partial F}{\partial x_{i}} \frac{\partial H}{\partial x_{i}}+\frac{\partial F}{\partial x_{i}} \frac{\partial h^{\prime}}{\partial x_{i}}+\frac{\partial Y^{\prime}}{\partial x_{i}} \frac{\partial H}{\partial x_{i}}+\frac{\partial Y^{\prime}}{\partial x_{i}} \frac{\partial h^{\prime}}{\partial x_{i}}=0
$$


The ensemble mean of $(2.5)$ is

$$
\left\langle\frac{\partial^{2} H}{\partial x_{i}^{2}}\right\rangle+\left\langle\frac{\partial F}{\partial x_{i}} \frac{\partial H}{\partial x_{i}}\right\rangle+\left\langle\frac{\partial F}{\partial x_{i}} \frac{\partial h^{\prime}}{\partial x_{i}}\right\rangle+\left\langle\frac{\partial Y^{\prime}}{\partial x_{i}} \frac{\partial H}{\partial x_{i}}\right\rangle+\left\langle\frac{\partial Y^{\prime}}{\partial x_{i}} \frac{\partial h^{\prime}}{\partial x_{i}}\right\rangle=0
$$

To simplify (2.5) and (2.6) it is assumed that the mean gradient $J_{i} \equiv-\partial H / \partial x_{i}, i=$ $1, \cdots, E$, where $E$ is the Euclidean space dimension, is constant (uniform) [Bakr et al., 1978, equation 16; Gelhar and Axness, 1983a, p. 163; Gelhar, 1986, equation 10]. This implies the absence of sinks or sources and the presence of constant mean head boundaries which maintain a constant mean hydraulic gradient vector $\boldsymbol{J}$. Equations 2.5 and 2.6 now simplify to

$$
\begin{gathered}
\frac{\partial^{2} h^{\prime}}{\partial x_{i}^{2}}+\frac{\partial F}{\partial x_{i}} \frac{\partial H}{\partial x_{i}}+\frac{\partial F}{\partial x_{i}} \frac{\partial h^{\prime}}{\partial x_{i}}+\frac{\partial Y^{\prime}}{\partial x_{i}} \frac{\partial H}{\partial x_{i}}+\frac{\partial Y^{\prime}}{\partial x_{i}} \frac{\partial h^{\prime}}{\partial x_{i}}=0 \\
\frac{\partial H}{\partial x_{i}}\left\langle\frac{\partial F}{\partial x_{i}}\right\rangle+\left\langle\frac{\partial F}{\partial x_{i}} \frac{\partial h^{\prime}}{\partial x_{i}}\right\rangle+\left\langle\frac{\partial Y^{\prime}}{\partial x_{i}} \frac{\partial h^{\prime}}{\partial x_{i}}\right\rangle=0 .
\end{gathered}
$$

Equation 2.8 can be further simplified by assuming that $\partial F / \partial x_{i}=$ constant $(i=$ $1, \cdots, E$; stationarity will later imply $\left.\partial F / \partial x_{i}=0\right)$,

$$
\frac{\partial H}{\partial x_{i}} \frac{\partial F}{\partial x_{i}}+\left\langle\frac{\partial Y^{\prime}}{\partial x_{i}} \frac{\partial h^{\prime}}{\partial x_{i}}\right\rangle=0
$$

Subtracting (2.9) from (2.7) we obtain [Gelhar, 1986, equation 8]

$$
\frac{\partial^{2} h^{\prime}}{\partial x_{i}^{2}}+\frac{\partial F}{\partial x_{i}} \frac{\partial h^{\prime}}{\partial x_{i}}+\frac{\partial H}{\partial x_{i}} \frac{\partial Y^{\prime}}{\partial x_{i}}=\left\langle\frac{\partial Y^{\prime}}{\partial x_{i}} \frac{\partial h^{\prime}}{\partial x_{i}}\right\rangle-\frac{\partial Y^{\prime}}{\partial x_{i}} \frac{\partial h^{\prime}}{\partial x_{i}}
$$

At this stage it is assumed that if the log hydraulic conductivity perturbations $Y^{\prime}$ are small (have a variance $\sigma_{Y}^{2} \ll 1$ ), then the products of their derivatives with those of the head perturbations $h^{\prime}$ would also be small and the right hand side of $(2.10)$ can be neglected [Bakr et al., 1978; Gelhar and Axness, 1983a; Gelhar, 1986]. Equation 2.10 then becomes [Gelhar, 1986, equation 9],

$$
\frac{\partial^{2} h^{\prime}}{\partial x_{i}^{2}}+\frac{\partial F}{\partial x_{i}} \frac{\partial h^{\prime}}{\partial x_{i}}+\frac{\partial H}{\partial x_{i}} \frac{\partial Y^{\prime}}{\partial x_{i}} \approx 0 .
$$


The supposition that smallness of the perturbed quantities $Y^{\prime}$ and $h^{\prime}$ implies smallness of the product of their derivatives has been questioned by Cushman [1983a] (see also reply by Gelhar and Axness [1983b]). The linearized expression for the effective hydraulic conductivity obtained by means of this approach was shown to be a good approximation to the exact nonlinear $\boldsymbol{K}_{e}$ only for $\sigma_{Y}^{2} \ll 1$ [Gutjahr and Gelhar, 1981; Dagan, 1982a; Gutjahr, 1984; Dagan, 1985]. Otherwise, as $\sigma_{Y}^{2}$ grows beyond 1, the exact $\boldsymbol{K}_{e}$ deviates from the linear approximation exponentially (Figures 2.2 and 3.1) [Dykaar and Kitanidis, 1992b; Neuman et al., 1992]. The governing equation for the perturbations of head and $Y$ is finally obtained from (2.11) by invoking stationarity of $\ln K$ [Bakr et al., 1978, equation 17; Gelhar and Axness, 1983a, equation 26; Naff and Vecchia, 1986, equation 1; Rubin and Dagan, 1988, equation 3],

$$
\frac{\partial^{2} h^{\prime}}{\partial x_{i}^{2}}=J_{i} \frac{\partial Y^{\prime}}{\partial x_{i}}
$$

To this point, the small perturbation approach has employed the assumptions of uniform mean hydraulic gradient, stationarity of $Y$, and mild heterogeneity which, in one form or another, are common to most stochastic theories. We proceed now to show that an additional assumption is imposed by the small perturbation and spectral approach, that of stationarity of the fluctuation of head and its gradient, which we will see later is not shared by (among others) the residual flux theory. As will be revealed in Chapter 3, it is precisely this assumption which renders the theory described in this subsection unsuitable for addressing problems of bounded domains.

Let us express Darcy's law in terms of the quantities in (2.3) and (2.4) [Gelhar and Axness, 1983a, equation 48] as

$$
q_{i}=-K \frac{\partial h}{\partial x_{i}}=-K_{G}\left(1+Y^{\prime}+\frac{Y^{\prime 2}}{2}+\cdots\right)\left(\frac{\partial H}{\partial x_{i}}+\frac{\partial h^{\prime}}{\partial x_{i}}\right)
$$




$$
\text { for } i=1, \cdots, E \text {, }
$$

where $K_{G}=\exp \langle Y\rangle$, the geometric mean of hydraulic conductivities. Taking the ensemble mean of (2.13) and dropping terms beyond second order (which holds at best for $\sigma_{Y}^{2}<1$ ) yields [Gutjahr et al., 1978, equation 33; Gelhar and Axness, 1983a, equation 49]

$$
\begin{array}{r}
\left\langle q_{i}\right\rangle=-K_{G}\left\{\frac{\partial H}{\partial x_{i}}\left(1+\frac{\sigma_{Y}^{2}}{2}\right)+\left\langle Y^{\prime} \frac{\partial h^{\prime}}{\partial x_{i}}\right\rangle\right\} \\
\text { for } i=1, \cdots, E
\end{array}
$$

One can cast (2.14) in the form

$$
\begin{array}{r}
\left\langle q_{i}\right\rangle=-K_{G}\left\{\left(1+\frac{\sigma_{Y}^{2}}{2}\right)+\left\langle Y^{\prime} \frac{\partial h^{\prime}}{\partial x_{i}}\right\rangle / \frac{\partial\langle h\rangle}{\partial x_{i}}\right\} \frac{\partial\langle h\rangle}{\partial x_{i}} \\
\text { for } i=1, \cdots, E
\end{array}
$$

reminiscent of (1.7). To evaluate (2.15), and consequently $\boldsymbol{K}_{e}$, the terms $\left\langle Y^{\prime} \partial h^{\prime} / \partial x_{i}\right\rangle$ $(i=1, \cdots, E)$ are evaluated from $(2.12)$ via a spectral approach that considers the head fluctuation to be stationary. Taking the Fourier Transform (FT) of (2.12), with the FT pair defined in (B.1) and (B.2), and utilizing relationships (C.7) and (C.10), one obtains

$$
d Z_{h^{\prime}}=\frac{i k_{i} J_{i}}{k^{2}} d Z_{Y^{\prime}}
$$

where $k^{2}=k_{i}^{2}$, the squared magnitude (Euclidean norm) of an E-dimensional wavenumber vector with components $k_{i}$, and $d Z_{h^{\prime}}=F T\left(h^{\prime}\right) d \boldsymbol{k}, d Z_{Y^{\prime}}=F T\left(Y^{\prime}\right) d \boldsymbol{k}$. Equation 2.16 has been obtained by Bakr et al. [1978, equation 19], Gelhar and Axness [1983a, equation 27] and Gelhar [1986, equation 10]. There is a difference in sign between (2.16) and their equations due to our definition of the FT pair [Bakr 
et al., 1978, equation 18] (opposite sign in the exponent of (B.1) which gives rise to a positive sign in (C.10)).

Let us now set $m_{i}=\partial h^{\prime} / \partial x_{i}(i=1, \cdots, E)$. The cross-covariance $C_{Y^{\prime} m_{i}}=$ $\left\langle Y^{\prime} \partial h^{\prime} / \partial x_{i}\right\rangle$ is given by

$$
\begin{array}{r}
C_{Y^{\prime} m_{i}}=\frac{1}{(2 \pi)^{E / 2}} \int_{\boldsymbol{k}} S_{Y^{\prime} m_{i}}(\boldsymbol{k}) e^{-i \boldsymbol{k} \cdot \boldsymbol{\chi}} d \boldsymbol{k} \\
i=1, \cdots, E,
\end{array}
$$

where $S_{Y^{\prime} m_{i}}(\boldsymbol{k})$, the cross-spectrum (the FT of the cross-covariance) of $Y^{\prime}$ and $\partial h^{\prime} / \partial x_{i}(i=1, \cdots, E)$. For $u(\boldsymbol{\chi})$ in (B.1) stationary, the "spectral representation theorem" ensures that the following relationships hold [Dagan, 1989, equations 1.6.14],

$$
\begin{array}{ll}
\left\langle d Z_{u}(\boldsymbol{k}) d Z_{u}^{*}\left(\boldsymbol{k}^{\prime}\right)\right\rangle=0 & \text { for } \boldsymbol{k} \neq \boldsymbol{k}^{\prime} \\
\left\langle d Z_{u}(\boldsymbol{k}) d Z_{u}^{*}(\boldsymbol{k})\right\rangle=S_{u u}(\boldsymbol{k}) d \boldsymbol{k} & \text { for } \boldsymbol{k}=\boldsymbol{k}^{\prime} .
\end{array}
$$

Here, $d Z_{u}=F T(u) d \boldsymbol{k}, d Z_{u}^{*}$ is its complex conjugate, and the spectral density (or spectrum) $S_{u u}(\boldsymbol{k})$ is the FT of the autocovariance of $u(\boldsymbol{\chi})$. At this point, the use of the representation theorem requires assuming that head perturbations and their gradients are stationary. With this, using (2.18)-(2.19) one can write

$$
\begin{aligned}
S_{Y^{\prime} m_{i}}(\boldsymbol{k}) d \boldsymbol{k} & =\left\langle d Z_{Y^{\prime}} d Z_{m_{i}}^{*}\right\rangle=\left\langle d Z_{Y^{\prime}} i k_{i} d Z_{h^{\prime}}^{*}\right\rangle \\
& =\left\langle d Z_{Y^{\prime}} i k_{i}\left(-\frac{i k_{j} J_{j}}{k^{2}}\right) d Z_{Y^{\prime}}^{*}\right\rangle \\
& =\frac{k_{i} k_{j} J_{j}}{k^{2}}\left\langle d Z_{Y^{\prime}} d Z_{Y^{\prime}}^{*}\right\rangle=\frac{k_{i} k_{j} J_{j}}{k^{2}} S_{Y Y}(\boldsymbol{k}) d \boldsymbol{k}
\end{aligned}
$$

where $i, j=1, \cdots, E$, and summation over the index $j$ is implied. The second and third equalities were obtained by taking the complex conjugate of equation $d Z_{m_{i}}=$ $-i k_{i} d Z_{h^{\prime}}(i=1, \cdots, E)$, obtained through (B.1), and of (2.16), respectively. Using 
(2.20), equation 2.17 becomes for $\boldsymbol{\chi}=0$ [Gutjahr et al., 1978, p. 956; Gelhar and Axness, 1983a, p. 166; Gelhar, 1986, equation 13]

$$
C_{Y^{\prime} m_{i}}=\left\langle Y^{\prime} \partial h^{\prime} / \partial x_{i}\right\rangle=\frac{J_{j}}{(2 \pi)^{E / 2}} \int_{\boldsymbol{k}} \frac{k_{i} k_{j}}{k^{2}} S_{Y Y}(\boldsymbol{k}) d \boldsymbol{k} .
$$

Expression 2.21 is an odd function in $k_{i}(i=1, \cdots, E)$ and, due to integration over a symmetric interval, can be simplified to

$$
\begin{array}{r}
C_{Y^{\prime} m_{i}}=\frac{J_{i}}{(2 \pi)^{E / 2}} \int_{\boldsymbol{k}} \frac{k_{i}^{2}}{k^{2}} S_{Y Y}(\boldsymbol{k}) d \boldsymbol{k} \\
i=1, \cdots, E .
\end{array}
$$

Substituting in (2.15) one finally obtains [Gutjahr et al., 1978, p. 956; Gelhar and Axness, 1983a, p. 166]

$$
\begin{array}{r}
\left\langle q_{i}\right\rangle=-K_{G}\left\{\left(1+\frac{\sigma_{Y}^{2}}{2}\right)-\frac{1}{(2 \pi)^{E / 2}} \int_{\boldsymbol{k}} \frac{k_{i}^{2}}{k^{2}} S_{Y Y}(\boldsymbol{k}) d \boldsymbol{k}\right\} \frac{\partial\langle h\rangle}{\partial x_{i}} \\
i=1, \cdots, E .
\end{array}
$$

For the one-dimensional case $(E=1)$ the second term within braces in $(2.23)$ equals, by definition, $\sigma_{Y}^{2}$ and hence [Gutjahr et al., 1978, equation 32],

$$
K_{e}^{L}=K_{H}^{L}=K_{G}\left[1-\sigma_{Y}^{2} / 2\right] \quad 1-\mathrm{D} .
$$

Here, $K_{H}^{L}$ is the linearized expression for the harmonic mean, the exact onedimensional infinite domain solution being $K_{e}=K_{H}=K_{G} e^{-\sigma_{Y}^{2} / 2}$ [Bear, 1972, p. 154; Gutjahr et a., 1978, equations 29, 30; Dagan, 1982a, equation 76]. For two- or three-dimensional flow ( $E=2$ or $E=3$ ) the sum of the integral terms in (2.23) yields $\sigma_{Y}^{2}$, and if in addition $Y^{\prime}$ is a statistically isotropic field, they equal each other. Thus, from (2.23), for statistically isotropic porous media, one easily obtains [Gutjahr et al., 1978; Gelhar and Axness, 1981, 1983a; Gelhar, 1986],

$$
K_{e}=K_{G} \quad 2-\mathrm{D},
$$




$$
K_{e}^{L}=K_{G}\left[1+\sigma_{Y}^{2} / 6\right] \quad 3-\mathrm{D}
$$

Matheron [1967] was the first to prove (2.25) rigorously for an infinite twodimensional, statistically isotropic log hydraulic conductivity field of any variance $\sigma_{Y}^{2}$. Matheron's method is presented in Neuman and Orr [1993] and will not be repeated here. He, as Shvidler [1962] before him, employed an asymptotic approximation and Green's function approach which is valid for infinite flow domains and resembles closely the subsequent analysis by Dagan [1989] which is discussed extensively in the next section. Shvidler and Matheron, like Landau and Lifshitz [1960] in the context of electrodynamics, conjectured that in an infinite isotropic field

$$
K_{e}=K_{G} \exp \left[\sigma_{Y}^{2}\left(\frac{1}{2}-\frac{1}{E}\right)\right]
$$

A rigorous proof for the three-dimensional $(E=3)$ result has recently been reported by Noetinger [1990]. Equation 2.27 for $E=3$ has been verified numerically by Dykaar and Kitanidis [1992b], and Neuman et al. [1992], for $\sigma_{Y}^{2}$ up to 6 and 7, respectively (Figures 2.2 and 3.1). Hence (2.27) is a generalization of (2.24)-(2.26) for arbitrary large $\sigma_{Y}^{2}$.

For statistically anisotropic media the general expression (2.23) for the effective hydraulic conductivity, initially derived by Gutjahr et al. [1978], was evaluated further by Gelhar and Axness [1981, 1983a] using an exponential, geometrically anisotropic covariance function. Gelhar and Axness [1981, 1983a] rewrote (2.23) in a system of coordinates oriented parallel to the principal directions of statistical anisotropy [Gelhar and Axness, 1983a, equation 52],

$$
K_{e_{i j}}=K_{G}\left[\left(1+\frac{\sigma_{Y}^{2}}{2}\right) \delta_{i j}-F_{i j}\right] \quad ; \quad F_{i j}=\sigma_{Y}^{2} \beta_{m i} \beta_{n j} g_{m n}
$$


Here $\beta_{i j}(i, j=1, \cdots, E)$ is the matrix of direction cosines between the two coordinate systems, and $g_{m n}$ are the integrals of (2.22) (divided by $\sigma_{Y}^{2}$ ) expressed in the new system. The integrals $g_{m n}$ were evaluated in spherical coordinates which reduced the triple integral to a single one [Gelhar and Axness, 1981, Appendix G], and subsequently by numerical integration. For a two-dimensional flow system the matrix $\boldsymbol{\beta}$ is given by

$$
\boldsymbol{\beta}=\left(\begin{array}{cc}
\cos \theta & -\sin \theta \\
\sin \theta & \cos \theta
\end{array}\right)
$$

where $\theta$ is the angle between the direction of mean flow and the first principal axis of the autocovariance of $Y$. The integrals $g_{m n}$ were expressed in closed form as [Gelhar and Axness, 1983, equation 55]

$$
g_{11}=\frac{\lambda_{2}}{\lambda_{1}+\lambda_{2}} \quad g_{22}=\frac{\lambda_{1}}{\lambda_{1}+\lambda_{2}}
$$

and $g_{m n}=0$ for $m \neq n$. Here $\lambda_{i}(i=1,2)$ are the integral scales in the principal coordinate directions. Substituting (2.29) and (2.30) in (2.28) one obtains

$$
K_{e_{i i}}=K_{G}\left[1+\frac{\sigma_{Y}^{2}}{2}-\sigma_{Y}^{2}\left(\frac{\lambda_{j} \cos ^{2} \theta+\lambda_{i} \sin ^{2} \theta}{\lambda_{1}+\lambda_{2}}\right)\right] \begin{array}{r}
i, j=1,2 \\
\text { and } i \neq j,
\end{array}
$$

and

$$
K_{e_{i j}}=K_{G} \sigma_{Y}^{2} \cos \theta \sin \theta \frac{\lambda_{2}-\lambda_{1}}{\lambda_{1}+\lambda_{2}} \quad \text { for } i \neq j .
$$

When the directions of the mean flow and the first principal axis of the covariance function coincide (or equivalently when $\boldsymbol{K}_{e}$ is expressed in the principal directions of the covariance function) $\theta=0$, then (2.31) reduces to

$$
\begin{aligned}
& K_{e_{i \mathfrak{i}}}=K_{G}\left[1+\frac{\sigma_{Y}^{2}}{2}-\frac{\lambda_{j} \sigma_{Y}^{2}}{\lambda_{1}+\lambda_{2}}\right] \quad i, j=1,2 \\
& \text { and } i \neq j \text {, }
\end{aligned}
$$


and (2.32) equals zero. This derivation of a closed form two-dimensional anisotropic result for $\boldsymbol{K}_{e}$ based on the work of Gelhar and Axness [1983a] was brought to our attention by Sanchez-Villa and Carrera [1993]; it has not been explicitly noted in the stochastic literature.

Gelhar and Axness [1983a, p. 167] proposed that in order to extend (2.28) to the domain of large $\sigma_{Y}^{2}$, it be considered as the first two terms in the Taylor series expansion of an exponential,

$$
\begin{array}{r}
K_{e_{i}}=K_{G} \exp \left[\sigma_{Y}^{2}\left(\frac{1}{2}-f_{i}\right)\right] \quad ; \quad f_{i}=\beta_{m i} \beta_{n i} g_{m n} \\
i=1,2,3 .
\end{array}
$$

A similar extension of the two-dimensional anisotropic result yields

$$
\begin{aligned}
K_{e_{i i}}=K_{G} \exp \left[\sigma_{Y}^{2}\left(\frac{1}{2}-\frac{\lambda_{j}}{\lambda_{1}+\lambda_{2}}\right)\right] \quad & i, j=1,2 \\
& \text { and } i \neq j .
\end{aligned}
$$

For a statistically isotropic medium both (2.33) and (2.35) reduce to the well known result $K_{e}=K_{G}$ [Matheron, 1967]. For perfectly stratified media with $\lambda_{i} \gg \lambda_{j}$ where $i, j=1,2$ and $i \neq j,(2.35)$ yields $K_{A}=K_{G} e^{\sigma_{Y}^{2} / 2}$, the arithmetic mean of hydraulic conductivities, and $K_{H}$, the harmonic mean, parallel and normal to the stratification, respectively.

Expression (2.35) has been attributed in the stochastic literature [Desbarats, 1992] to Ababou [1991] (see Section on power-averaging method). Desbarats [1992, Figure 7 and p. 264] found that, for a rectangular field with anisotropy ratio $\lambda_{x} / \lambda_{z}=$ 10, equation 2.35 does not agree well with numerical Monte Carlo results. We believe that this may be due to the small number of realizations (fewer than 25 according to Figure 7 of Desbarats [1992]) and, to a lesser degree, the narrow width of the flow 
field ( $L_{x}=30 \lambda_{z}$ and $L_{z}=3 \lambda_{z}$ ) which suggests that no-flow boundaries affect the computed effective hydraulic conductivity [Rubin and Dagan, 1989], or, equivalently, that this flow field does not quite approximate an infinite domain.

Neuman and Depner [1988, p. 487] and Dagan [1989, p. 192] have shown independently that (2.34) does not depend on the choice of covariance function but only on $\sigma_{Y}^{2}$ and the ratios between the integral scales, $\lambda_{i} / \lambda_{j}(i, j=1,2,3)$. The former authors have further verified (2.34) against field data from fractured crystalline rocks having $\sigma_{Y}^{2}$ in excess of 7 .

\subsubsection{Asymptotic Expansion and Green's Function Approach}

We return to the steady state flow equation (1.5) and its transformed form (1.6). One can formally expand $h(\boldsymbol{x})$ in an asymptotic sequence [Dagan, 1989, equation $3.3 .8]$

$$
h(\boldsymbol{x})=h^{(0)}(\boldsymbol{x})+h^{(1)}(\boldsymbol{x})+h^{(2)}(\boldsymbol{x})+\cdots
$$

such that the variance of each term forms an asymptotic sequence for $\sigma_{Y}^{2}$. Substituting (2.36) into (1.6) and solving it iteratively one obtains the sequence of equations [Dagan, 1989, equation 3.3.9]

$$
\begin{aligned}
\nabla^{2} h^{(0)}(\boldsymbol{x})= & 0 \quad ; \quad \nabla^{2} h^{(1)}(\boldsymbol{x})=-\nabla Y(\boldsymbol{x}) \cdot \nabla h^{(0)}(\boldsymbol{x}) \\
& \nabla^{2} h^{(2)}(\boldsymbol{x})=-\nabla Y(\boldsymbol{x}) \cdot \nabla h^{(1)}(\boldsymbol{x}), \cdots
\end{aligned}
$$

where $h^{(0)}(\boldsymbol{x})$ accounts for all boundary conditions. Hence all other $h^{(i)}(\boldsymbol{x}), i=$ $1,2, \cdots$, satisfy homogeneous conditions on the boundary $\Gamma$ of the flow domain $\Omega$. For deterministic (Dirichlet) boundary conditions it follows from $(2.37)$ that $h^{(0)}(\boldsymbol{x})$

is deterministic and hence $\left\langle h^{(0)}(\boldsymbol{x})\right\rangle=h^{(0)}(\boldsymbol{x})$. Furthermore, for $Y(\boldsymbol{x})$ stationary, 
one has

$$
\nabla^{2}\left\langle h^{(1)}(\boldsymbol{x})\right\rangle=-\nabla\langle Y(\boldsymbol{x})\rangle \cdot \nabla h^{(0)}(\boldsymbol{x})=0 .
$$

Since $\left\langle h^{(1)}(\boldsymbol{x})\right\rangle$ satisfies homogeneous boundary conditions, it follows [Dagan, 1989, equations 3.3.10, 3.4.8] that $\left\langle h^{(1)}(\boldsymbol{x})\right\rangle=0$.

Dagan [1989, equation 3.4.2] considers deterministic boundary conditions such that

$$
h(\boldsymbol{x})=-\boldsymbol{J} \cdot \boldsymbol{x} \quad \text { for } \boldsymbol{x} \in \Gamma,
$$

where $\boldsymbol{J}$ is a constant vector. Since $h^{(0)}(\boldsymbol{x})$ satisfies (2.37) and accounts for all nonhomogeneous boundary conditions, it follows that $h^{(0)}(\boldsymbol{x})=-\boldsymbol{J} \cdot \boldsymbol{x}$. Under this condition, the second equation in (2.37) becomes identical to (2.12). Taking the ensemble mean of (2.37) yields [Neuman, 1992, p. 227], for a stationary $Y(\boldsymbol{x})$,

$$
\begin{aligned}
\nabla^{2}\left\langle h^{(2)}(\boldsymbol{x})\right\rangle= & -\left\langle\nabla Y(\boldsymbol{x}) \cdot \nabla h^{(1)}(\boldsymbol{x})\right\rangle=-\left\langle\nabla\langle Y(\boldsymbol{x})\rangle \cdot \nabla h^{(1)}(\boldsymbol{x})\right\rangle \\
& -\left\langle\nabla Y^{\prime}(\boldsymbol{x}) \cdot \nabla h^{(1)}(\boldsymbol{x})\right\rangle \\
= & -\nabla \cdot\left\langle Y^{\prime}(\boldsymbol{x}) \nabla h^{(1)}(\boldsymbol{x})\right\rangle+\left\langle Y^{\prime}(\boldsymbol{x}) \nabla^{2} h^{(1)}(\boldsymbol{x})\right\rangle \\
= & -\nabla \cdot\left\langle Y^{\prime}(\boldsymbol{x}) \nabla h^{(1)}(\boldsymbol{x})\right\rangle-\left\langle Y^{\prime}(\boldsymbol{x}) \nabla Y^{\prime}(\boldsymbol{x}) \cdot \nabla h^{(0)}(\boldsymbol{x})\right\rangle \\
= & -\nabla \cdot\left\langle Y^{\prime}(\boldsymbol{x}) \nabla h^{(1)}(\boldsymbol{x})\right\rangle+\frac{1}{2} \nabla\left\langle Y^{\prime 2}(\boldsymbol{x})\right\rangle \cdot \boldsymbol{J} \\
= & -\nabla \cdot\left\langle Y^{\prime}(\boldsymbol{x}) \nabla h^{(1)}(\boldsymbol{x})\right\rangle+\nabla \frac{\sigma_{Y}^{2}}{2} \cdot \boldsymbol{J} \\
= & -\nabla \cdot\left\langle Y^{\prime}(\boldsymbol{x}) \nabla h^{(1)}(\boldsymbol{x})\right\rangle .
\end{aligned}
$$

Dagan [1989, equation 3.4 .8$]$ sets (2.40) equal to zero, namely

$$
\nabla \cdot\left\langle Y^{\prime}(\boldsymbol{x}) \nabla h^{(1)}(\boldsymbol{x})\right\rangle=\left\langle\nabla Y(\boldsymbol{x}) \cdot \nabla h^{(1)}(\boldsymbol{x})\right\rangle=0,
$$

so that (2.40), subject to homogeneous boundary conditions, gives

$$
\left\langle h^{(2)}(\boldsymbol{x})\right\rangle=0
$$


The right hand side of (2.41) implies that, on the average, the gradients of head and $\log$ hydraulic conductivity perturbations are orthogonal to each other. In flow domains of infinite extent $h^{(1)}$ is stationary and hence $\left\langle Y^{\prime}(\boldsymbol{x}) \nabla h^{(1)}(\boldsymbol{x})\right\rangle$ is constant, rendering (2.41) correct. However, in bounded domains $h^{(1)}$ is nonstationary and hence $\left\langle Y^{\prime}(\boldsymbol{x}) \nabla h^{(1)}(\boldsymbol{x})\right\rangle$ is not a constant (see Section 3.2.2), so that the divergence in (2.40) is generally not equal to zero and (2.42) is incorrect (contrary to an explicit assertion by Dagan).

From Darcy's law the flux is

$$
\begin{aligned}
\boldsymbol{q}(\boldsymbol{x}) & =-K(\boldsymbol{x}) \nabla h(\boldsymbol{x})=-K_{G} e^{Y^{\prime}} \nabla h(\boldsymbol{x}) \\
& =-K_{G}\left[1+Y^{\prime}+\frac{Y^{\prime 2}}{2}+\cdots\right]\left[\nabla h^{(0)}+\nabla h^{(1)}+\nabla h^{(2)}+\cdots\right],
\end{aligned}
$$

where $Y^{\prime}=Y-\langle Y\rangle$ are zero mean random fluctuations in $Y(\boldsymbol{x})$. If the flux is also expanded in asymptotic series

$$
\boldsymbol{q}=\boldsymbol{q}^{(0)}+\boldsymbol{q}^{(1)}+\boldsymbol{q}^{(2)}+\cdots
$$

then (2.43) can be written [Dagan, 1989, equation 3.4.7] as

$$
\begin{gathered}
\boldsymbol{q}^{(0)}=-K_{G} \nabla h^{(0)} \\
\boldsymbol{q}^{(1)}=-K_{G}\left\{Y^{\prime} \nabla h^{(0)}+\nabla h^{(1)}\right\} \\
\boldsymbol{q}^{(2)}=-K_{G}\left\{\frac{Y^{\prime 2}}{2} \nabla h^{(0)}+Y^{\prime} \nabla h^{(1)}+\nabla h^{(2)}\right\} \\
\vdots
\end{gathered}
$$

Taking ensemble means of (2.45) yields, to first order in $\sigma_{Y}^{2}$ [Dagan, 1989, equation 3.4.9],

$$
\left\langle\boldsymbol{q}^{(0)}\right\rangle=K_{G} \boldsymbol{J} ;\left\langle\boldsymbol{q}^{(1)}\right\rangle=0 ;\left\langle\boldsymbol{q}^{(2)}\right\rangle=K_{G}\left\{\frac{\sigma_{Y}^{2}}{2} \boldsymbol{J}-\left\langle Y^{\prime} \nabla h^{(1)}\right\rangle\right\},
$$


or

$$
\langle\boldsymbol{q}\rangle=K_{G}\left\{\left(1+\frac{\sigma_{Y}^{2}}{2}\right) \boldsymbol{J}-\boldsymbol{\alpha}\right\} ; \boldsymbol{\alpha}=\left\langle Y^{\prime} \nabla h^{(1)}\right\rangle .
$$

When equalities (2.41) and (2.42) are not valid, as in the case of a bounded domain, $\left\langle\boldsymbol{q}^{(2)}\right\rangle$ and $\langle\boldsymbol{q}\rangle$ include another term $-K_{G} \nabla\left\langle h^{(2)}\right\rangle$. We show in Chapter 3 that this term is indeed recovered by Neuman and Orr [1993].

To evaluate $\boldsymbol{\alpha}$, Dagan rewrites the second equation in (2.37) as

$$
\nabla^{2} h^{(1)}(\boldsymbol{\chi})-\nabla Y^{\prime}(\boldsymbol{\chi}) \cdot \boldsymbol{J}=0
$$

multiplies by the Green's function (fundamental solution) $G_{\infty}(\boldsymbol{x}, \boldsymbol{\chi})$ which satisfies

$$
\nabla^{2} G_{\infty}(\boldsymbol{x}, \boldsymbol{\chi})+\delta(\boldsymbol{\chi}-\boldsymbol{x})=0
$$

in an infinite domain, and integrates over $\Omega_{\infty}$ to obtain [Dagan, 1989, equation 3.4.11]

$$
\begin{aligned}
h^{(1)}(\boldsymbol{x}) & =-\boldsymbol{J} \cdot \int_{\Omega_{\infty}} \nabla_{\chi} Y^{\prime}(\boldsymbol{\chi}) G_{\infty}(\boldsymbol{\chi}, \boldsymbol{x}) d \boldsymbol{\chi} \\
& =\boldsymbol{J} \cdot \int_{\Omega_{\infty}} Y^{\prime}(\boldsymbol{\chi}) \nabla_{\chi} G_{\infty}(\boldsymbol{\chi}, \boldsymbol{x}) d \boldsymbol{\chi}
\end{aligned}
$$

Taking the derivative of $(2.50)$ with respect to $x_{i}(i=1, \cdots, E)$, multiplying by $Y^{\prime}(\boldsymbol{x})$ and taking ensemble mean gives

$$
\alpha_{i}=\boldsymbol{J} \cdot \int_{\Omega_{\infty}}\left\langle Y^{\prime}(\boldsymbol{x}) Y^{\prime}(\boldsymbol{\chi})\right\rangle \frac{\partial}{\partial x_{i}} \nabla_{\chi} G_{\infty}(\boldsymbol{\chi}, \boldsymbol{x}) d \boldsymbol{\chi}
$$

Equations 2.50 and 2.51 might seem to hold for a bounded domain $\Omega$ upon replacement of the fundamental solution $G_{\infty}$ by the corresponding Green's function. However, as already mentioned, the expression for $\boldsymbol{K}_{e}$ in (2.47) is incomplete for a bounded domain [Neuman, 1992, p. 229]. Using Parseval's identity (Appendix B) 
Dagan [1989, equation 3.4.13] obtains (for a detailed derivation refer to Section 3.3 and Appendix F)

$$
\begin{array}{r}
\alpha_{i}=\frac{\boldsymbol{J}}{(2 \pi)^{\frac{E}{2}}} \cdot \int_{\boldsymbol{k}} \frac{\boldsymbol{k} k_{i}}{k^{2}} S_{Y Y}(\boldsymbol{k}) d \boldsymbol{k} \\
i=1, \cdots, E .
\end{array}
$$

Equations 2.47 and 2.52 are identical to 2.14 and 2.21 derived via the small perturbation and spectral approach. Hence the procedure of subsection 2.1 .1 can be used for further evaluation of $\boldsymbol{K}_{e}$.

\subsubsection{Method of Embedding Matrix}

Dagan [1979, 1981] for statistically homogeneous and isotropic fields, and Poley [1988] and Dagan [1989] for anisotropic fields, applied the method of embedding matrix to derive expressions for $\boldsymbol{K}_{e}$ that are said by the authors to hold for arbitrarily large variances of $Y(\boldsymbol{x})$ and not to require a normality assumption. This method considers a uniform background medium with randomly embedded non overlapping inclusions having a different uniform hydraulic conductivity. The method assumes that any disturbance in the head field caused by an inclusion in this binary medium is not affected by similar disturbances due to other inclusions. For sand-shale formations characterized by a bimodal permeability distribution with a large variance, (2.28) gave aberrant results whereas Dagan's [1979] expression

$$
K_{e}=\frac{1}{E}\left[\int \frac{f(K) d K}{(E-1) K_{e}+K}\right]^{-1}
$$

was found to agree fairly well with numerical results [Desbarats, 1987a,b]. Here, $f(K)$ is the frequency distribution of $K(\boldsymbol{x})$. One should recall however that $(2.28)$ was developed for a unimodal, not a bimodal, distribution. Indeed, Dykaar and 
Kitanidis [1992b], Figure 2.2, found that, for lognormally distributed $K(\boldsymbol{x})$ fields (2.53) yields predictions farther from the exact result (2.27) than does the linearized approximation (2.26). They concluded that for such media the assumption of non interacting isolated inclusions is not appropriate; the interaction between spatial variations in $K(\boldsymbol{x})$ is significant.

\subsubsection{Method of Moments for Periodic Media}

The method of moments for periodic media [Kitanidis, 1990] is considered by some [Fenton and Griffiths, 1993] to provide $\boldsymbol{K}_{e}$ for a bounded domain. We present below details of this approach in order to show that it was developed assuming infinite flow domains.

Kitanidis [1990, p. 1197] states the following (the quotation is verbatim except for equation numbers): "Consider transient flow in a saturated porous medium. In the absence of sources or sinks, the governing equation is given by

$$
\nabla \cdot(\boldsymbol{K} \nabla \phi)=S \frac{\partial \phi}{\partial t}
$$

where $\phi$ is the piezometric head $[L] ; S$ is the specific storage coefficient $\left[L^{-1}\right]$, which in the analysis will be assumed constant; $\boldsymbol{K}$ is the hydraulic conductivity $[L / T]$, a symmetric and positive definite second-order tensor (positive definite meaning that the conductivity is positive in every direction); and $t$ denotes time $[T]$. To keep the analysis simple, assume that the boundary condition is that

$$
\phi(\boldsymbol{x}, t)=0 \quad \text { for very large } \boldsymbol{x}
$$

and the initial condition is that of a "slug injection"

$$
\phi(\boldsymbol{x}, 0)=\delta\left(\boldsymbol{x}-\boldsymbol{x}^{\prime}\right)
$$


where $\delta$ is a Dirac delta function. That is, a "unit volume" increase in the piezometric head was introduced at time 0 at location $\boldsymbol{x}^{\prime}$. A point of clarification: In these equations, $\phi$ should be interpreted as the head above a background level, $\phi_{b}$, which satisfies the governing equation (2.54) and is subject to prescribed steady boundary conditions. For example, $\phi_{b}\left(x_{1}, x_{2}, x_{3}, t\right)=J x_{1}+c$, where $J$ is slope and $c$ is a constant. However, the background head is of no importance in this analysis. (A reviewer has suggested that $\phi$ can be seen as the drawdown resulting from a unitvolume slug withdrawal)."

This description reveals that the above transient flow probem, (2.54)-(2.56), is driven solely by the initial condition (2.56), the boundary condition set at infinity signifying a flow domain of infinite extent. This is consistent with the subsequent presentation of an analytical solution for $\phi$, for $\boldsymbol{K}$ constant in space, representing a bell-shaped mound in an unbounded domain [ibid, equation 4, and Figures 1-4], and the use of the notation $V_{\infty}$ [ibid, equation 5], "where $V_{\infty}$ signifies the entire space". Thus, it appears that the analysis applies only to infinite domains and does not consider boundary effects, as concurred by Kitanidis himself [1994]. However, in Section 5.4, we show that the solution for $K_{e}$ derived through this method appears to capture boundary effects. As such, though a theoretical justification is missing, it may be considered to provide $K_{e}$ in a bounded flow domain.

The introduction of a time-independent background head $\phi_{b}$ gives the impression that the author views $\phi$ as a perturbation around $\phi_{b}$, namely that the total head $h$ can be decomposed as $h=\phi_{b}+\phi$. The total head satisfies

$$
\nabla \cdot(\boldsymbol{K} \nabla h)=S \frac{\partial h}{\partial t}
$$


which can also be written as

$$
\nabla \cdot\left(\boldsymbol{K} \nabla \phi_{b}\right)+\nabla \cdot(\boldsymbol{K} \nabla \phi)=S \frac{\partial \phi}{\partial t}
$$

Since $\phi$ satisfies (2.54), it follows from (2.58) that $\phi_{b}$ is given by

$$
\nabla \cdot\left(\boldsymbol{K} \nabla \phi_{b}\right)=0
$$

The solution $\phi_{b}=J x_{1}+c$, for $J \neq 0$, satisfies (2.59) only if $\partial \boldsymbol{K} / \partial x_{1}=0$, which is true if $\boldsymbol{K}$ is constant in the $x_{1}$ direction. Since $\boldsymbol{K}$ is considered later to be fully periodic in all directions, the corresponding result applies only to a uniform background head, $\phi_{b}=c$.

Kitanidis $[1990$, p. 1200$]$ considers $\boldsymbol{K}$ to vary periodically in all directions (and to be differentiable),

$$
\boldsymbol{K}\left(X_{1}, X_{2}, X_{3}\right)=\boldsymbol{K}\left(X_{1}+m_{1} l_{1}, X_{2}+m_{2} l_{2}, X_{3}+m_{3} l_{3}\right)
$$

where $l_{i}$ is the period in direction $i, i=1,2,3, \boldsymbol{X}$ are coordinates in a global Cartesian system, and $m_{1}, m_{2}$, and $m_{3}$ are integers (positive, negative, or zero). A grid of rectangular parallelepiped elements with lengths of sides equal to the periods $l_{i}(i=$ $1,2,3)$ is superimposed on the infinite domain. The origin of the global coordinate system $\boldsymbol{X}$ is taken (without loss of generality) to coincide with the center of an element where at time $t=0$ the excitation ocurrs. A system of local coordinates $\boldsymbol{x}$ is associated with each element. It is parallel to the global system, has its origin at the center of each element, and is related to $\boldsymbol{X}$ through

$$
X_{i}=n_{i} l_{i}+x_{i} \quad i=1,2,3 .
$$

Here, $\boldsymbol{n}=\left(n_{1}, n_{2}, n_{3}\right)$ (with $n_{i}$ integers) identifies the distance in periods between the centroid of an element and the global origin, and $-l_{i} / 2 \leq x_{i} \leq l_{i} / 2$. From the 
periodicity of $\boldsymbol{K}$, and the particular discretization of the domain, it follows that $\boldsymbol{K}$ at a given local coordinate $\boldsymbol{x}$ is the same in all elements. For the same reasons it follows that, in each element,

$$
\begin{aligned}
& \boldsymbol{K}\left(l_{1} / 2, x_{2}, x_{3}\right)=\boldsymbol{K}\left(-l_{1} / 2, x_{2}, x_{3}\right) \\
& \boldsymbol{K}\left(x_{1}, l_{2} / 2, x_{3}\right)=\boldsymbol{K}\left(x_{1},-l_{2} / 2, x_{3}\right) \\
& \boldsymbol{K}\left(x_{1}, x_{2}, l_{3} / 2\right)=\boldsymbol{K}\left(x_{1}, x_{2},-l_{3} / 2\right),
\end{aligned}
$$

and the same (periodic) conditions hold for the gradient of $\boldsymbol{K}$. In this way, the heterogeneity of an infinite periodic medium is completely described by that of a single element.

Within each element, $\phi$ is governed by equation (2.54) where the gradient operator $\nabla$ is considered with respect to the local coordinates. Local boundary conditions now result from the requirements of continuity of head and flux at the interface between any two adjacent elements (this does not imply periodic boundary conditions for $\phi)$. The boundary condition at infinity, (2.55), translates into

$$
\phi(\boldsymbol{n}, \boldsymbol{x}, t)=0 \quad \text { as } \boldsymbol{n} \rightarrow \pm \infty,
$$

i.e., for elements far away from the singularity $\phi$ is zero. The initial condition (2.56) becomes

$$
\phi(\boldsymbol{n}, \boldsymbol{x}, 0)=\delta\left(\boldsymbol{n} \boldsymbol{l}^{T}+d \boldsymbol{x}\right),
$$

where $\boldsymbol{l}=\left(l_{1}, l_{2}, l_{3}\right)$ and $d \boldsymbol{x}=\boldsymbol{x}-\boldsymbol{x}^{\prime}$. Equation 2.64 means that for all elements except the one in which the excitation occurs, $\phi(\boldsymbol{x}, 0)=0$. In this singular element the same relationship is true for all local points $\boldsymbol{x} \neq \boldsymbol{x}^{\prime}$, at $\boldsymbol{x}^{\prime}$ such that $\phi\left(\boldsymbol{x}^{\prime}, 0\right)=\delta(0)$. For large times the above problem reduces to solving, for any element, the 
boundary value problem

$$
\nabla \cdot\left[\boldsymbol{K}(\boldsymbol{x}) \nabla g^{i}(\boldsymbol{x})\right]=\nabla \cdot \boldsymbol{\kappa}_{i}(\boldsymbol{x}) \quad i=1, \cdots, E
$$

where, $\boldsymbol{\kappa}_{i}$ is the $\mathrm{i}$-th column of $\boldsymbol{K}$, and the solution $g^{i}$ and its derivatives satisfy periodic boundary conditions. The periodicity of the boundary conditions, for $g^{i}$ and its derivatives, arise from the assumption that the head and its derivatives vanish at large distances [Kitanidis, 1990, pp. 1202-1204]. By inserting the auxiliary functions $g^{i}$ in the integral equation

$$
K_{i j}^{e}=-\frac{1}{2 V} \int_{V}\left(\boldsymbol{\kappa}_{i} \cdot \nabla g^{j}+\boldsymbol{\kappa}_{j} \cdot \nabla g^{i}\right) d \boldsymbol{x}+\tilde{K}_{i j} \quad i, j=1, \cdots, E,
$$

one obtains the effective conductivity for periodic media in infinite flow domains. Here, $\tilde{K}_{i j}$ is the spatial average of $K_{i j}$ over the volume of a local element $V=l_{1} l_{2} l_{3}$. To extend (2.66) to random media Kitanidis [1990, p. 1204] states (the quotation is verbatim and is emphasized by us): "Consider that $\boldsymbol{D}=\boldsymbol{K} / S$ is not truly periodic but still varies about a mean value $\overline{\boldsymbol{D}}$ in a "stationary" fashion (The assumption of stationary variability underlies every attempt to define effective properties). Despite the fact that the medium is not truly periodic, one can still superimpose a regular grid and apply the methodology using the $D$ values over one element. One can then solve the associated boundary value problems and calculate the integral. If the size of the grid is large enough, it is quite possible that the value of the volume integral of (2.66) will tend to a constant value. This value will be practically independent of the spacing of the grid and of which element was used in the computation."

For locally isotropic ( $\boldsymbol{K}=K \boldsymbol{I}$, with $\boldsymbol{I}$ the identity matrix) lognormally distributed media with $\sigma_{Y}^{2}<1,(2.66)$ becomes [ibid, equation 80 ]

$$
K_{i j}^{e}=K_{G}\left(1+\frac{\sigma_{Y}^{2}}{2}\right) \delta_{i j}-K_{G} \sum_{\boldsymbol{k}} \frac{k_{i} k_{j}}{k^{2}} S_{Y Y}(\boldsymbol{k}) \quad i, j=1, \cdots, E,
$$


where $S_{Y Y}(\boldsymbol{k})$ is the power spectrum of $Y, \boldsymbol{k}$ is an $\mathrm{E}$-dimensional wave number vector, and $\delta_{i j}$ is the Kronecker delta taking values $\delta_{i j}=1$ for $i=j$, and $\delta_{i j}=0$ for $i \neq j$. When $V \rightarrow \infty$ (or equivalently the period tends to infinity) the multiple summation in (2.67) becomes a multiple integral and one obtains the expression for $K_{i j}^{e}$ in (2.23), developed via stochastic theories.

The Fourier Galerkin numerical method [Dykaar and Kitanidis, 1992a] was used to solve equations 2.65 and to evaluate $(2.66) . K(\boldsymbol{x})$ and $g^{i}(\boldsymbol{x})$ were expanded in truncated Fourier series, the Fourier coefficients of $K(\boldsymbol{x})$ were approximated by their discrete Fourier coefficients, and the discrete Fourier coefficients of $g^{i}(\boldsymbol{x})$ were obtained by inserting both series in (2.65), then setting the Fourier transform of the residual of this equation equal to zero.

Dykaar and Kitanidis [1992b], Figure 2.1, found that (2.67) converged numerically to the two- and three- dimensional infinite domain results, (2.25) and (2.26), when the period $l$ exceeded 20 and 10 integral scales, respectively. In accordance with the analysis of Matheron [1967], Gutjahr et al. [1978], and Gelhar [1986], they showed that the effect on $K^{e}$ from the use of an isotropic exponential and Gaussian input covariance is minimal (Neuman and Depner [1988] and Dagan [1989] have shown $\boldsymbol{K}_{e}$ to be independent of the autocovariance even for anisotropic media). The full solution, (2.66), for an isotropic Gaussian covariance function and higher variances $\left(\sigma_{Y}^{2}=2\right.$ and $\left.\sigma_{Y}^{2}=4\right)$ converged in two-dimensions to $K_{G}$ as the period approached about 80 integral scales (10 realizations) [ibid, Figures 4 and 5]. For the three-dimensional case, for an isotropic Gaussian covariance function and $\sigma_{Y}^{2}=4$ (3 realizations), approximately 30 integral scales were required for (2.66) to reach (2.27) [ibid, Figure 7]. Finally, the full solution (2.66) was shown, Figure 2.2, to be in excellent agreement 
with (2.27), Matheron's conjecture. The same figure also shows the self-consistent method giving worse predictions than the small perturbation approach. Neuman et al. [1992] found independently, Figure 3.1, (2.27) to be in excellent agreement with a numerical solution generated by an isotropic exponential covariance.

\subsubsection{Ad Hoc Power-Averaging Method}

Journel et al. [1986] suggested that the effective hydraulic conductivity can be described by the power averaging formula

$$
K_{e}=K_{\omega}=\left[\frac{1}{E} \sum_{i=1}^{E} K_{i}^{\omega}\right]^{1 / \omega}
$$

which corresponds to the $\omega$-norm of an $E$-dimensional vector with components $K_{i}$. Ababou and Wood [1990a, equation 6] developed a closed form expression for the $\omega$-norm for stationary Gaussian fields $Y(\boldsymbol{x})$. In an effort to provide a theoretical justification for the power averaging approach, Ababou [1991] cast it in the form

$$
K_{\omega}=K_{G} \exp \left[\sigma_{Y}^{2}(1 / 2-p)\right]
$$

which is reminiscent of (2.27) and (2.34). Whereas (2.27) and (2.34) are based on analyses of the flow equation, Ababou's (2.69) is strictly conjectural. He set $p=(1-\omega) / 2$ where $\omega$ is to be established by numerical experiments as done by Deutsch [1989], Gómez-Hernández and Gorelick [1989], and Desbarats [1992a]. For infinite domains, Ababou [1991] conjectured that

$$
\omega_{i}=1-\frac{2 \lambda_{H}}{E \lambda_{i}} \quad i=1, \ldots, E
$$

where

$$
\lambda_{H}=\left(\frac{1}{E} \sum_{i=1}^{E} \lambda_{i}^{-1}\right)^{-1}
$$




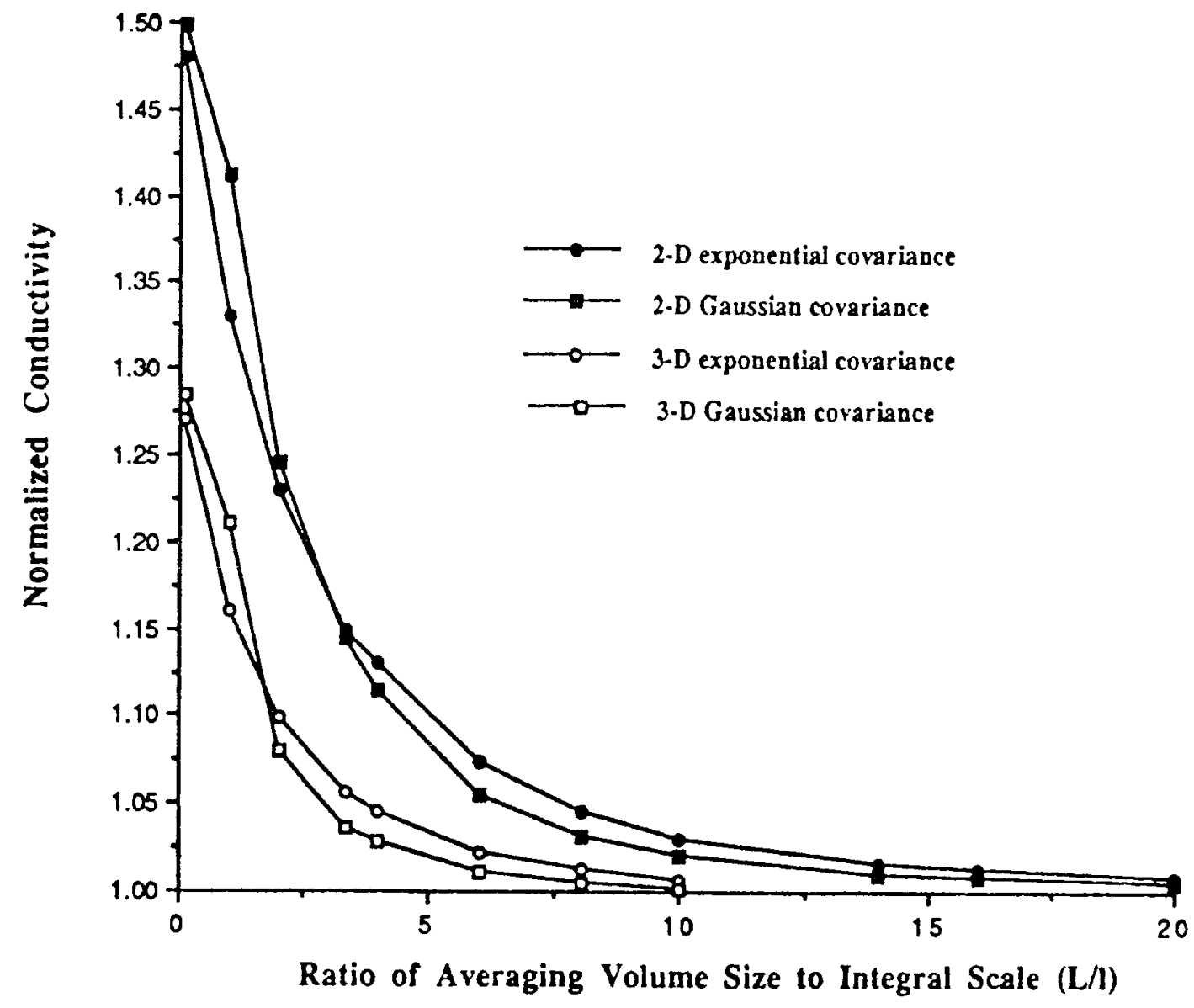

Figure 2.1: Dykaar and Kitanidis [1992b]: Numerical evaluation of (2.67) for isotropic exponential and Gaussian input covariances. 


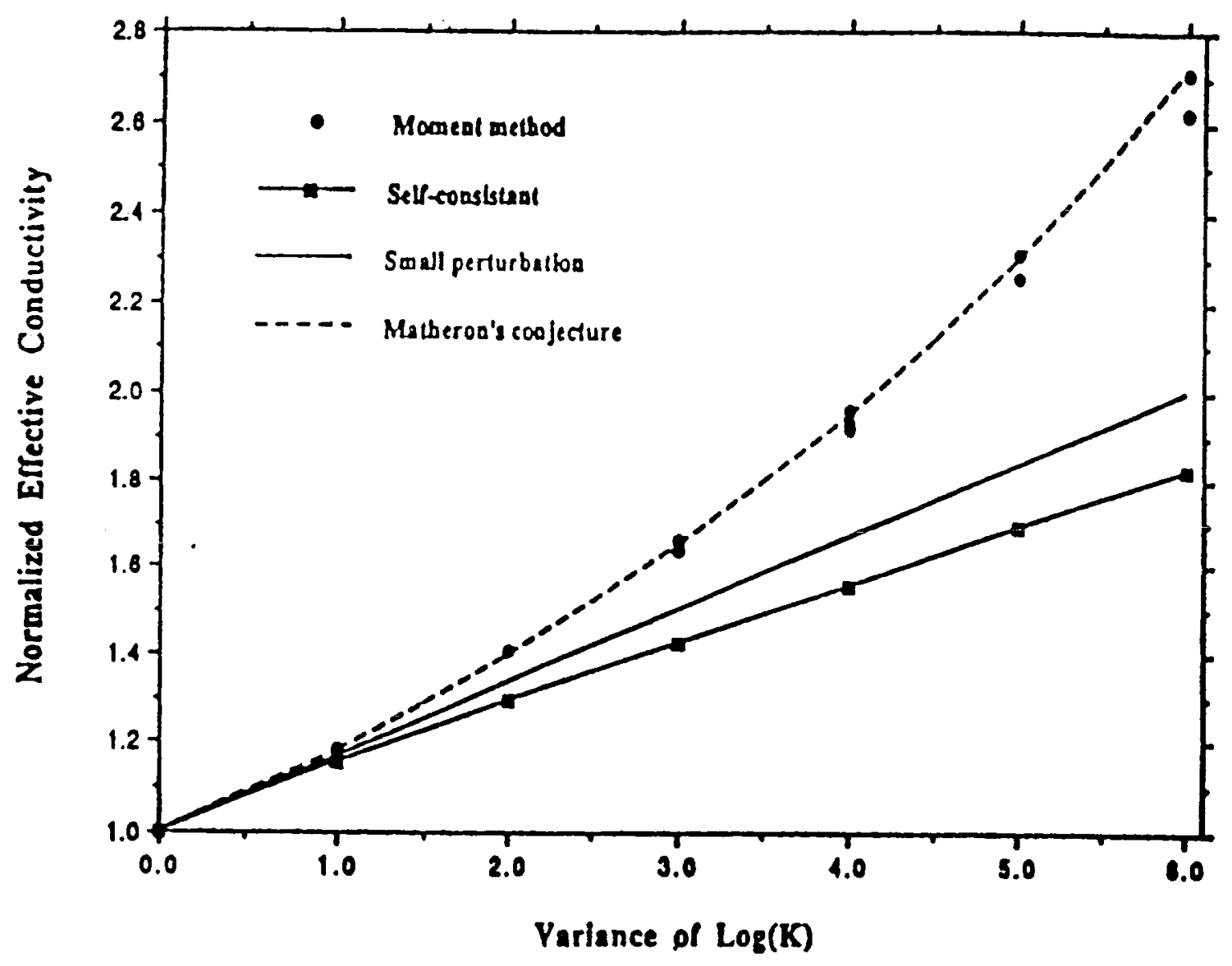

Figure 2.2: Dykaar and Kitanidis [1992b]: Comparison between (2.26), (2.27), (2.53), and numerical $K_{e}$. 
Substituting (2.70)-(2.71) into (2.69) yields the following form for $\boldsymbol{K}_{e}$ in anisotropic two-dimensional infinite domains,

$$
\begin{aligned}
K_{e_{i i}}=K_{G} \exp \left[\sigma_{Y}^{2}\left(\frac{1}{2}-\frac{\lambda_{j}}{\lambda_{1}+\lambda_{2}}\right)\right] \quad & i, j=1,2 \\
& \text { and } i \neq j
\end{aligned}
$$

which had previously been developed by Gelhar and Axness [1983a], equation 2.35, via a small perturbation and spectral analysis of the flow equation. Equation 2.72 will also be derived formally (Appendix J) via the residual flux theory, and the asymptotic expansion and Green's function approach of Dagan [1989] as applied by Sanchez-Villa and Carrera [1993].

\subsection{Bounded Domain Solutions}

In this section we review results for $\boldsymbol{K}_{e}$ in bounded domains obtained via numerical methods. Analytical studies concerned with the effects of boundaries on moments of head are also discussed to provide a perspective on the range of influence of these boundaries.

\subsubsection{Small Perturbation and Green's Function Approach}

Naff and Vecchia [1986] investigated the effects of no-flow boundaries on the head covariance function. They employed a small perturbation, equation 2.12, and Green's function approach, equation 2.50 (with appropriate Green's function), to study steady state three-dimensional flow domains bounded by two infinite impervious horizontal planes, and subject to a constant mean hydraulic gradient in the $x_{1}$ di-

rection. $Y(\boldsymbol{x})$ was assumed to be a second-order stationary random field, described 
by a covariance function exhibiting a negative exponential behavior in the vertical direction and a "hole effect" in the horizontal plane. This form of input covariance was required to keep the head variance finite. Naff and Vecchia [1986, Figures 2-5] found that both the head variance and the head covariance exhibit nonstationary effects resulting from the imposition of boundary conditions.

Rubin and Dagan [1988] addressed the effects of a constant head boundary on the semivariogram $\Gamma_{h}$ of head $h$, and the cross covariance $C_{Y h}$ of head and $Y(\boldsymbol{x})=\ln T(\boldsymbol{x})$. They studied steady state two-dimensional flow in a semi-infinite domain subject to a constant mean head gradient normal to the boundary via (2.12) and (2.50) (with appropriate Green's function). $Y(\boldsymbol{x})$ was assumed normally distributed, statistically homogeneous and isotropic, and was described by a separated exponential covariance function. Rubin and Dagan [1988, p. 1692] found that, at distances greater than 4 integral scales from the boundary, both $\Gamma_{h}$ and $C_{Y h}$ can be adequately described by their corresponding infinite domain expressions.

In a companion paper, Rubin and Dagan [1989] showed that the effects of an impervious boundary in a two-dimensional flow domain under constant mean head gradient parallel to the boundary become negligible at distances greater than 3 integral scales from the no-flow boundary.

\subsubsection{Numerical Studies via Monte Carlo Simulation}

Smith and Freeze [1979b] studied two-dimensional steady state flow in a rectangular aquifer subject to a uniform mean hydraulic head gradient. The hydraulic conductivity field was assumed to be lognormally distributed, statistically homogeneous and isotropic. Realizations of $Y(\boldsymbol{x})=\log K(\boldsymbol{x})$ were generated by a first-order nearest- 
neighbor model. The authors found both the total flux through the system and the flux across specified blocks on the inflow-outflow boundaries to be accurately predicted by $K_{G}$. Details of their numerical experiments regarding $K_{e}$ were not provided. However, the largest value of $\sigma_{Y}$ utilized in this study (which was primarily an analysis of uncertainty in hydraulic head) was 0.91 . Their results were calculated from 300 simulations, and the flow domain had dimensions $L_{x}=200$ and $L_{y}=100$ units [Smith and Freeze, 1979b, p. 1548]. The range of values of integral scales, throughout the study, was between 10 and 26 units. Thus, $a_{x}$, the ratio of the distance between constant head boundaries and integral scale, assumed values between 8 and 20 , whereas $a_{y}$, the distance between no-flow boundaries and integral scale, varied between 4 and 10 .

Several researchers [Desbarats and Dimitrakopoulos, 1990; Rubin and GómezHernández, 1990; Desbarats, 1992a; Fenton and Griffiths, 1993] have investigated the conditions under which an effective parameter can be approximated by a blockaveraged value. In these studies, an expression for the block-averaged conductivity (transmissivity) is often proposed a priori (without reference to the flow equation), and then its relationship to the effective parameter is studied via a Monte Carlo analysis. This conjectured expression is usually of the kind described by (2.68), selected in such a way as to provide correct limit behavior. In all cases, the effective conductivity is defined for one numerical simulation, and thus only its ensemble mean corresponds to our definition of effective parameter in (1.7). The authors took $Y(\boldsymbol{x})$ to be normally distributed, statistically homogeneous and isotropic, and possessing an exponential covariance function.

Desbarats and Dimitrakopoulos [1990] addressed the relationship between 
$T_{S}$, a block-averaged transmissivity, and $T_{e}$, the effective transmissivity, in a twodimensional steady state bounded flow domain for $\sigma_{Y}^{2}=1.324$. $T_{S}$ was defined as a spatial geometric average of the point transmissivities $T(\boldsymbol{x})$ over a block of area $S=L^{2}$

$$
T_{S}=\exp \left[\frac{1}{S} \int_{S} Y(\boldsymbol{x}) d \boldsymbol{x}\right]
$$

$T_{e}$ was calculated numerically by solving the steady state flow equation and determining the total flux through the system. $T_{S}$ was found to be a good approximation for $T_{e}$ in fields of size $1 \lambda \times 1 \lambda$ and $5 \lambda \times 5 \lambda$ where $\lambda$ is the integral scale of $Y$. The sample mean of $T_{S}$ (from less than 70 simulations) approached $K_{G}$ when $L / \lambda>20$ [Desbarats and Dimitrakopoulos, 1990, Figures 5-8].

Rubin and Gómez-Hernández [1990] studied the problem of upscaling, or defining block-averaged transmissivities from point variables. The (block) effective transmissivity $T_{S}$ over an area $S$ was defined as

$$
T_{S}\left(\boldsymbol{x}^{\prime}\right)=\frac{1}{S} \int_{S} Q(\boldsymbol{x}) d \boldsymbol{x}\left(\frac{1}{S} \int_{S} \bar{J}(\boldsymbol{x}) d \boldsymbol{x}\right)^{-1}
$$

where $Q$ and $\bar{J}$ are integrated (over the aquifer thickness $L$ ) flux and head gradient, respectively, $\boldsymbol{x}$ is a two-dimensional space vector, and $\boldsymbol{x}^{\prime}$ is the centroid of $S$. Expression (2.74) was calculated numerically for a rectangular aquifer with constant head gradient in the longitudinal direction, and impervious boundaries in the transverse direction, respectively. Its mean over 200 Monte Carlo realizations (corresponding approximately to an effective parameter) for different size blocks, was compared to the linearized (only terms of order smaller than 2 were retained in their analysis) expression [Rubin and Gómez-Hernández, 1990, equation 17]

$$
\left\langle T_{S}\right\rangle=T_{G}\left[1+\frac{1}{J_{1} S^{2}} C_{Y d H}(S, S)+\cdots\right] .
$$


Here $J_{1}$ is a constant unidirectional mean head gradient, and $C_{Y d H}(S, S)$ is the crosscovariance of $Y$ and the derivative of head in the direction of $J_{1}$, regularized over $S$. $Y(\boldsymbol{x})=\ln T(\boldsymbol{x})$ was assumed multivariate normal with constant ensemble mean. In (2.75) $C_{Y d H}$ was approximated by its infinite expression [ibid, p. 694]. The authors found that the applicability of (2.75) improved with an increase in the size of $S$ which in addition counterweighted any increase in $\sigma_{Y}^{2}$. At block sizes greater than 6 integral scales, for $\sigma_{Y}^{2}$ up to $2,(2.75)$ agreed very well with the ensemble mean of (2.74) [ibid, Figures 8-11].

Desbarats [1992a] studied the relationship between $K_{V}$, a block-averaged conductivity, and $K_{e}$, the effective conductivity in a cubic field of size $L$. Steady state flow was considered, with constant head and no-flow boundaries applied in the longitudinal and transverse directions of the field, respectively. $K_{V}$ was defined as a spatial "power average" of the point random function $K(\boldsymbol{x})$ over the volume of the block $V$,

$$
K_{V}=\left(\frac{1}{V} \int_{V} K(\boldsymbol{x})^{\omega} d V\right)^{\frac{1}{\omega}}
$$

with a constant $\omega=1 / 3$ utilized in the numerical experiments [Desbarats, 1992a, equation 21]. $K_{e}$ was calculated as the ratio of the longitudinal flux across a transverse section of the field over the applied (constant) gradient and the cross-sectional area. For 200 realizations and $\sigma_{Y}^{2}=1.0$, Desbarats [1992a, Figure 1, Table I, equations 5 and 14] found that the sample mean of $K_{V}$ approached the three-dimensional infinite domain solution (2.27) at $a=L / \lambda=10$. For $a=0.1$, the sample mean of $K_{V}$ approached $K_{A}=K_{G} \exp \left(\sigma_{Y}^{2} / 2\right)$, the arithmetic mean of hydraulic conductivities. A very good agreement (in less than 25 simulations) between $K_{V}$ and $K_{e}$ was seen to exist for fixed $a=3$ and $\sigma_{Y}^{2}$ up to 2.0 [Desbarats, 1992a, Figure 3A and B], as 
well as for different ratios $a$ at $\sigma_{Y}^{2}=1$ [Desbarats, 1992a, Figure 4A, B, and C]. However, this agreement deteriorated noticeably for higher values of $\sigma_{Y}^{2}$ [Desbarats, 1992a, Figure 3C].

Fenton and Griffiths [1993] studied two-dimensional steady state flow in a rectangular aquifer with a constant applied mean head gradient in the longitudinal direction, and impervious boundaries in the transverse direction, respectively. Three different isotropic exponential correlation functions $\rho_{Y}$ were employed. The block conductivity $\bar{K}$ of the whole domain, calculated for each realization by computing the total flow rate through the system, was found (from 2000 simulations) to be (similarly to the point conductivities) lognormally distributed. Its moments were calculated as functions of the statistics of the point variable $Y=\ln K$. The estimated mean, $m_{\ln \bar{K}}$, of $\ln \bar{K}$, normalized by the statistics $\mu_{\ln K}$ and $\sigma_{\ln K}^{2}$ of $\ln K$, is shown in Figure 2.3 for the three $\rho_{Y}$, for different aspect ratios $X_{L} / Y_{L}$ of horizontal to vertical length of the domain. The correlation scale $\theta$ was normalized by the length $D=\left(X_{L} Y_{L}\right)^{1 / 2}$. Figure 2.3 demonstrates the dependence of $m_{\ln \bar{K}}$ on the dimensions of the flow domain (both in terms of aspect ratio and relative to correlation scale) and the input correlation function. 

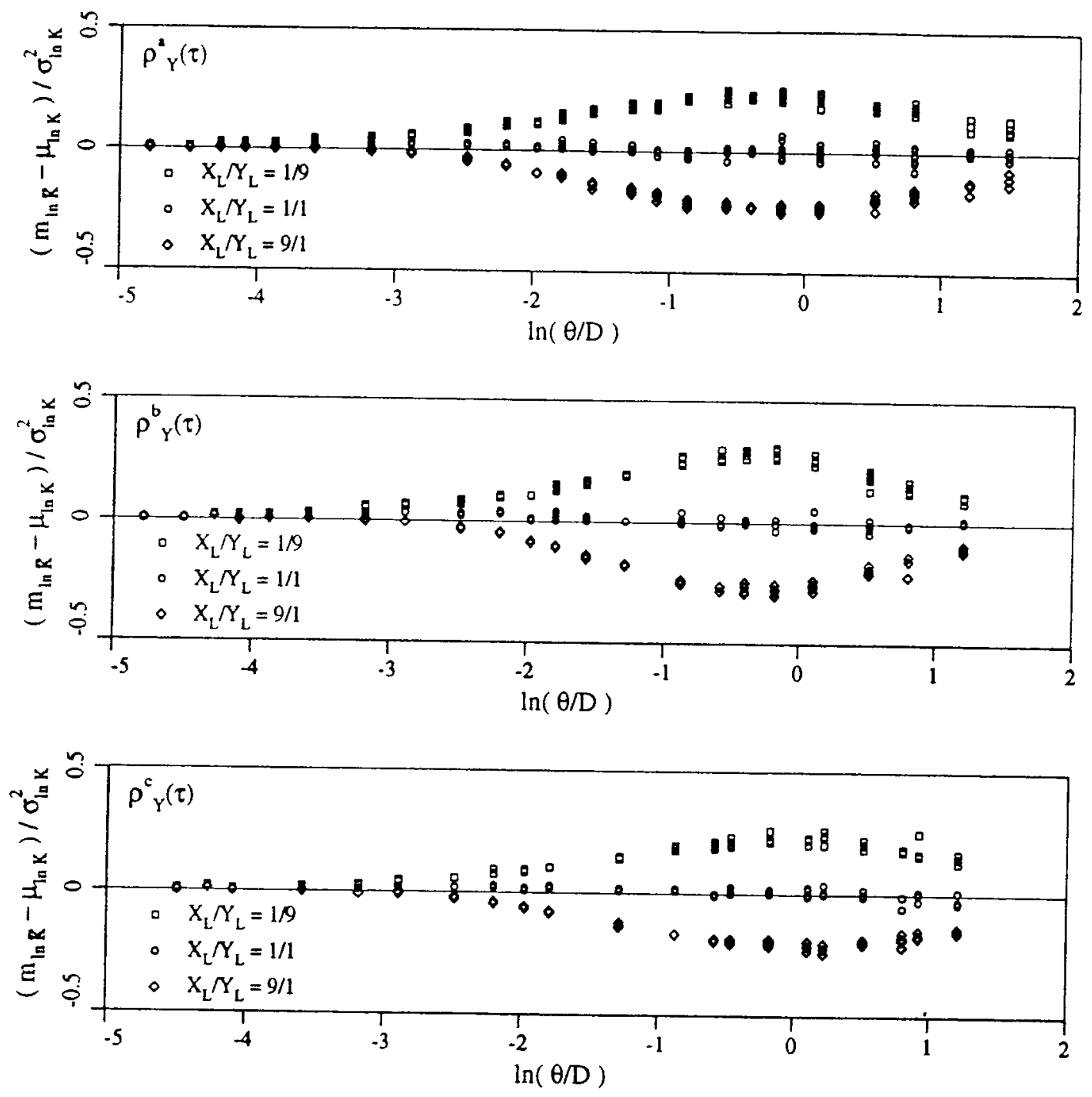

Figure 2.3: Fenton and Griffiths [1993]: Block conductivity in rectangular domain. 


\section{CHAPTER 3}

\section{RESIDUAL FLUX THEORY}

The basic elements of the residual flux theory of Neuman and Orr [1993] are described in this chapter to facilitate the presentation of subsequent analyses concerning effective hydraulic conductivity. This theory is compared in section 3.2 to other stochastic theories dealing with bounded domains. In the last section of this chapter we verify that the theory replicates known results for infinite 3 - and 2-dimensional flow domains under a uniform mean hydraulic gradient.

\subsection{Basic Equation for the Residual Flux}

The theory starts from the premise that if Darcy's law

$$
\boldsymbol{q}(\boldsymbol{x})=-K(\boldsymbol{x}) \nabla h(\boldsymbol{x})
$$

can be found to be experimentally valid on a support $\omega$ which is small compared to the flow domain $\Omega$, then this law can be regarded as a local constitutive relationship. Here $\boldsymbol{q}(\boldsymbol{x}), K(\boldsymbol{x})$, and $\nabla h(\boldsymbol{x})$ are representative of the support volume $\omega$ centered about some point $\boldsymbol{x}$, and $K(\boldsymbol{x})$ can be evaluated locally at points $\boldsymbol{x}$ with the aid of existing measurement and interpretive methods. Upon combination with the principle of mass conservation one obtains an operational theory of groundwater flow [Baveye and Sposito, 1984, 1985] that is unambiguously tied to the measurement methods 
used and the specific scale of heterogeneity detected by the measurement process, a need clearly pointed out by Cushman [1983b, 1984, 1986, 1987, 1993].

The steady state continuity equation

$$
-\nabla \cdot \boldsymbol{q}(\boldsymbol{x})+f(\boldsymbol{x})=0 \quad \text { for } \boldsymbol{x} \in \Omega
$$

is considered subject to the boundary conditions

$$
\begin{array}{ll}
h(\boldsymbol{x})=H(\boldsymbol{x}) & \text { for } \boldsymbol{x} \in \Gamma_{D}, \\
-\boldsymbol{q}(\boldsymbol{x}) \cdot \boldsymbol{n}(\boldsymbol{x})=Q(\boldsymbol{x}) & \text { for } \boldsymbol{x} \in \Gamma_{N} .
\end{array}
$$

Here $f(\boldsymbol{x})$ is a randomly prescribed source function, $H(\boldsymbol{x})$ and $Q(\boldsymbol{x})$ are randomly prescribed head and flux on the Dirichlet and Neumann boundary segments $\Gamma_{D}$ and $\Gamma_{N}$, respectively, and $\boldsymbol{n}(\boldsymbol{x})$ is a unit outward normal on the total boundary $\Gamma=\Gamma_{D} \cup \Gamma_{N}$. The prescribed source, head and flux are considered, for simplicity, to be statistically independent of each other. The treatment of boundary conditions as random reflects the common scarcity of data on aquifers boundaries [Gelhar, 1986].

The residual flux theory of Neuman and Orr [1993] is conditional in that it incorporates formally the effect of measuring $K(\boldsymbol{x})$ at specific points $\boldsymbol{x}$. The development in this thesis is unconditional (the locations of hydraulic conductivity data are not taken into account).

In the conditional theory, the hydraulic conductivity field is decomposed according to

$$
K(\boldsymbol{x})=\kappa(\boldsymbol{x})+K^{\prime}(\boldsymbol{x})
$$

where $\kappa(\boldsymbol{x})=\langle K(\boldsymbol{x})\rangle$, a deterministic smooth function, and $K^{\prime}(\boldsymbol{x})$, hydraulic conductivity perturbations about $\kappa(\boldsymbol{x})$ with ensemble mean $\left\langle K^{\prime}(\boldsymbol{x})\right\rangle \equiv 0$. Similarly, the 
hydraulic head $h(\boldsymbol{x})$ and flux $\boldsymbol{q}(\boldsymbol{x})$ are written as

$$
\begin{aligned}
& h(\boldsymbol{x})=\langle h(\boldsymbol{x})\rangle+h^{\prime}(\boldsymbol{x}), \\
& \boldsymbol{q}(\boldsymbol{x})=\langle\boldsymbol{q}(\boldsymbol{x})\rangle+\boldsymbol{q}^{\prime}(\boldsymbol{x}),
\end{aligned}
$$

with the perturbations $h^{\prime}(\boldsymbol{x})$ and $\boldsymbol{q}^{\prime}(\boldsymbol{x})$ defined to have zero ensemble means, $\left\langle h^{\prime}(\boldsymbol{x})\right\rangle \equiv 0$ and $\left\langle\boldsymbol{q}^{\prime}(\boldsymbol{x})\right\rangle \equiv 0$.

Taking ensemble means of (3.2)-(3.4) gives

$$
-\nabla \cdot\langle\boldsymbol{q}(\boldsymbol{x})\rangle+\langle f(\boldsymbol{x})\rangle=0 \quad \text { for } \boldsymbol{x} \in \Omega,
$$

subject to the boundary conditions

$$
\begin{array}{ll}
\langle h(\boldsymbol{x})\rangle=\langle H(\boldsymbol{x})\rangle & \text { for } \boldsymbol{x} \in \Gamma_{D}, \\
-\langle\boldsymbol{q}(\boldsymbol{x})\rangle \cdot \boldsymbol{n}(\boldsymbol{x})=\langle Q(\boldsymbol{x})\rangle & \text { for } \boldsymbol{x} \in \Gamma_{N},
\end{array}
$$

which shows that $\langle\boldsymbol{q}(\boldsymbol{x})\rangle$ obeys a standard continuity equation driven by ensemble mean source and boundary functions.

The ensemble mean of Darcy's law (3.1) is

$$
\begin{aligned}
\langle\boldsymbol{q}(\boldsymbol{x})\rangle & =-\langle K(\boldsymbol{x}) \nabla h(\boldsymbol{x})\rangle \\
& =-\left\langle\left(\kappa(\boldsymbol{x})+K^{\prime}(\boldsymbol{x})\right) \nabla\left(\langle h(\boldsymbol{x})\rangle+h^{\prime}(\boldsymbol{x})\right)\right\rangle \\
& =-\kappa(\boldsymbol{x}) \nabla\langle h(\boldsymbol{x})\rangle-\left\langle K^{\prime}(\boldsymbol{x}) \nabla h^{\prime}(\boldsymbol{x})\right\rangle .
\end{aligned}
$$

Equation 3.11 consists of two terms, the first Darcian, the second, $\boldsymbol{r}(\boldsymbol{x})=$ $-\left\langle K^{\prime}(\boldsymbol{x}) \nabla h^{\prime}(\boldsymbol{x})\right\rangle$, called residual flux. 
For $\boldsymbol{r}(\boldsymbol{x})$ (and hence $\langle\boldsymbol{q}(\boldsymbol{x})\rangle$ ) to be Darcian, it is necessary that there exists a symmetric, positive-semidefinite second-rank tensor $\tilde{\boldsymbol{\kappa}}(\boldsymbol{x})$ which is additionally independent of $\langle h(\boldsymbol{x})\rangle$. Then the residual flux can be written

$$
\boldsymbol{r}(\boldsymbol{x})=\tilde{\boldsymbol{\kappa}}(\boldsymbol{x}) \nabla\langle h(\boldsymbol{x})\rangle,
$$

which in turn leads to

$$
\langle\boldsymbol{q}(\boldsymbol{x})\rangle=-\boldsymbol{K}_{e}(\boldsymbol{x}) \nabla\langle h(\boldsymbol{x})\rangle .
$$

The effective hydraulic conductivity $\boldsymbol{K}_{e}(\boldsymbol{x})$, obtained by substituting (3.12) into (3.11), is a symmetric, positive-definite second-rank tensor given by

$$
\boldsymbol{K}_{e}(\boldsymbol{x})=\kappa(\boldsymbol{x}) \boldsymbol{I}-\tilde{\boldsymbol{\kappa}}(\boldsymbol{x}),
$$

where $\boldsymbol{I}$ is the identity tensor. As shown by Neuman and Orr [1993], conditions for the existence of $\boldsymbol{K}_{e}(\boldsymbol{x})$ exist formally only in special circumstances. The range of conditions under which $\boldsymbol{K}_{e}(\boldsymbol{x})$ may exist as an approximation appears to be much broader.

The residual flux is given by [Neuman and Orr, 1993, equations 12, 19a, 19b, and 19c]

$$
\begin{aligned}
\boldsymbol{r}(\boldsymbol{x})= & \int_{\Omega}\left\langle K^{\prime}(\boldsymbol{x}) K^{\prime}(\boldsymbol{\chi}) \nabla_{x} \nabla_{\chi}^{T} \mathcal{G}(\boldsymbol{\chi}, \boldsymbol{x})\right\rangle \nabla_{\chi} h_{\kappa}(\boldsymbol{\chi}) d \boldsymbol{\chi} \\
- & \int_{\Gamma_{D}}\left\langle K^{\prime}(\boldsymbol{x}) K(\boldsymbol{\chi}) \nabla_{x} \nabla_{\chi}^{T} \mathcal{G}(\boldsymbol{\chi}, \boldsymbol{x})\right\rangle \boldsymbol{n}(\boldsymbol{\chi})\langle H(\boldsymbol{\chi})\rangle d \boldsymbol{\chi} \\
- & \int_{\Gamma_{N}}\left[\left\langle K^{\prime}(\boldsymbol{x}) K^{\prime}(\boldsymbol{\chi}) \nabla_{x} \mathcal{G}(\boldsymbol{\chi}, \boldsymbol{x})\right\rangle \kappa(\boldsymbol{\chi})^{-1}-\left\langle K^{\prime}(\boldsymbol{x}) \nabla_{x} \mathcal{G}(\boldsymbol{\chi}, \boldsymbol{x})\right\rangle\right] \\
& \langle Q(\boldsymbol{\chi})\rangle d \boldsymbol{\chi} .
\end{aligned}
$$

Here $\mathcal{G}(\boldsymbol{\chi}, \boldsymbol{x})$ is a random Green's function satisfying the stochastic Poisson equation

$$
\nabla_{\chi} \cdot\left[K(\chi) \nabla_{\chi} \mathcal{G}(\boldsymbol{\chi}, \boldsymbol{x})\right]+\delta(\boldsymbol{\chi}-\boldsymbol{x})=0 \quad \text { for } \boldsymbol{\chi}, \boldsymbol{x} \in \Omega
$$


subject to the homogeneous boundary conditions

$$
\begin{array}{ll}
\mathcal{G}(\boldsymbol{\chi}, \boldsymbol{x})=0 & \text { for } \boldsymbol{\chi} \in \Gamma_{D} \\
K(\boldsymbol{\chi}) \nabla_{\chi} \mathcal{G}(\boldsymbol{\chi}, \boldsymbol{x}) \cdot \boldsymbol{n}(\boldsymbol{\chi})=0 & \text { for } \boldsymbol{\chi} \in \Gamma_{N} .
\end{array}
$$

The deterministic function $h_{\kappa}(\boldsymbol{x})$ is defined as the solution of

$$
\nabla \cdot\left[\kappa(\boldsymbol{x}) \nabla h_{\kappa}(\boldsymbol{x})\right]+\langle f(\boldsymbol{x})\rangle=0 \quad \text { for } \boldsymbol{x} \in \Omega,
$$

with boundary conditions

$$
\begin{array}{cc}
h_{\kappa}(\boldsymbol{x})=\langle H(\boldsymbol{x})\rangle & \text { for } \boldsymbol{x} \in \Gamma_{D} \\
\kappa(\boldsymbol{x}) \nabla h_{\kappa}(\boldsymbol{x}) \cdot \boldsymbol{n}(\boldsymbol{x})=\langle Q(\boldsymbol{x})\rangle & \text { for } \boldsymbol{x} \in \Gamma_{N} .
\end{array}
$$

Equation 3.15 clearly shows that $\boldsymbol{r}(\boldsymbol{x})$ is generally not proportional to the local hydraulic gradient but depends, additionally, on deterministic head gradients at other points $\boldsymbol{\chi}$ of the flow domain. Thus, $\boldsymbol{r}(\boldsymbol{x})$ is in general nonlocal and non-Darcian.

As properties of the stochastic Green's function $\mathcal{G}(\boldsymbol{\chi}, \boldsymbol{x})$ are unknown, equation 3.15 can be evaluated only by Monte Carlo simulation or approximation. We evaluate (3.15) analytically by treating $K(\boldsymbol{x})$ as a homogeneous field with a constant mean $\kappa=\langle K(\boldsymbol{x})\rangle=$ constant. Then, under uniform mean flow parallel to a principal direction of statistical anisotropy, the conditions for the existence of $\boldsymbol{K}_{e}$ exist, the latter being a constant [Neuman and Orr, 1993]. To evaluate it, we approximate $\mathcal{G}(\boldsymbol{\chi}, \boldsymbol{x})$, equations 3.16 to 3.18 , to zeroth order by the deterministic Green's function $G_{d}(\boldsymbol{\chi}, \boldsymbol{x})$ defined via

$$
\nabla_{\chi} \cdot\left[\kappa(\chi) \nabla_{\chi} G_{d}(\boldsymbol{\chi}, \boldsymbol{x})\right]+\delta(\boldsymbol{\chi}-\boldsymbol{x})=0 \quad \text { for } \boldsymbol{\chi}, \boldsymbol{x} \in \Omega
$$


subject to the homogeneous boundary conditions

$$
\begin{array}{ll}
G_{d}(\boldsymbol{\chi}, \boldsymbol{x})=0 & \text { for } \chi \in \Gamma_{D} \\
\kappa(\boldsymbol{\chi}) \nabla_{\chi} G_{d}(\boldsymbol{\chi}, \boldsymbol{x}) \cdot \boldsymbol{n}(\boldsymbol{\chi})=0 & \text { for } \chi \in \Gamma_{N} .
\end{array}
$$

The residual flux $\boldsymbol{r}(\boldsymbol{x})$ is then given approximately by

$$
\begin{aligned}
\boldsymbol{r}(\boldsymbol{x}) & \approx \int_{\Omega}\left\langle K^{\prime}(\boldsymbol{x}) K^{\prime}(\boldsymbol{\chi})\right\rangle \nabla_{x} \nabla_{\chi}^{T} G_{d}(\boldsymbol{\chi}, \boldsymbol{x}) \nabla_{\chi} h_{\kappa}(\boldsymbol{\chi}) d \boldsymbol{\chi} \\
& -\int_{\Gamma_{D}}\left\langle K^{\prime}(\boldsymbol{x}) K^{\prime}(\boldsymbol{\chi})\right\rangle \nabla_{x} \nabla_{\chi}^{T} G_{d}(\boldsymbol{\chi}, \boldsymbol{x}) \boldsymbol{n}(\boldsymbol{\chi})\langle H(\boldsymbol{\chi})\rangle d \boldsymbol{\chi} \\
& -\int_{\Gamma_{N}}\left\langle K^{\prime}(\boldsymbol{x}) K^{\prime}(\boldsymbol{\chi})\right\rangle \nabla_{x} G_{d}(\boldsymbol{\chi}, \boldsymbol{x}) \kappa(\boldsymbol{\chi})^{-1}\langle Q(\boldsymbol{\chi})\rangle d \boldsymbol{\chi} .
\end{aligned}
$$

Since we take $K(\boldsymbol{x})$ to be statistically homogeneous and $\kappa(\boldsymbol{x})=\kappa=$ constant, we have $\left\langle K^{\prime}(\boldsymbol{x}) K(\boldsymbol{\chi})\right\rangle=\left\langle K^{\prime}(\boldsymbol{x}) K^{\prime}(\boldsymbol{\chi})\right\rangle$. Henceforth, $\boldsymbol{\chi}$ will designate a coordinate system oriented in the principal directions of the log hydraulic conductivity (say parallel and normal to the directions of stratification), and $\boldsymbol{x}$ will designate a singularity point in this system. Equation 3.25 is now amenable to analytical evaluation and, as such, forms the starting point for our subsequent analyses.

\subsection{Comparison of Residual Flux Theory to other Theories}

The residual flux theory is compared here to other stochastic theories for the case of bounded flow domains. We consider the approach of Section 2.1.1 as consisting of two distinct methods for the evaluation of $\boldsymbol{K}_{e}$. The first, the small perturbation approach, is used to derive the governing equation for the perturbations of head and $Y$. The second, the spectral approach, is utilized to evaluate a term, $\left\langle Y^{\prime} \partial h^{\prime} / \partial x_{i}\right\rangle$, which is part of the expression for $\boldsymbol{K}_{e}$. The latter imposes stationarity in head fluctuations and their gradient by invoking the spectral representation theorem. Similarly, 
Dagan [1989] resorts to two methods for the evaluation of $\boldsymbol{K}_{e}$. The first method consists of expanding head and flux in an asymptotic sequence and developing the governing equations for the terms of this sequence. The second, used to evaluate

$\boldsymbol{\alpha}=\left\langle Y^{\prime} \nabla h^{(1)}\right\rangle$, is the known mathematical technique of solving an equation by means of the adjoint operator (Green's function approach). The former imposes stationarity in head fluctuations and their gradient by considering $\alpha$ to be constant. We show in Section 3.2.1 that head fluctuations in bounded flow domains are nonstationary. This renders both theories unsuitable for addressing this class of flow problems. In Section 3.2.2 we discuss the case of uniform mean flow parallel to an impervious boundary where $\boldsymbol{\alpha}$, as conceded by Dagan [1989] himself, is not a constant. For this flow field we show that an extra term of order $O\left(\sigma_{Y}^{2}\right)$ has been neglected by Dagan [1989] in his expression for $\boldsymbol{K}_{e}$.

\subsubsection{Non-Stationarity of Head Fluctuations in Bounded Domains}

We investigate now the appropriateness of the assumption of stationarity of head fluctuations for steady state saturated flow under uniform mean hydraulic gradient in bounded domains. For one-dimensional flow through layers in series, Freeze [1975, pp. 730-732], Smith and Freeze [1979a, pp. 524-526], and Dagan [1979, p. 56; 1982a, p. 821] have shown that in general $\sigma_{h}^{2}$ depends on $x$, the heterogeneity of the medium, the integral scale of the log hydraulic conductivity, the boundary conditions, and the extent of the flow domain. Stationary solutions for the head field will occur only for special forms of the input log hydraulic conductivity covariance function [Bakr et al., 1978]. For two-dimensional flow, $\sigma_{h}^{2}(\boldsymbol{x})$ grows indefinitely in infinite domains [Dagan, 1981, pp. 116-118], or with the distance from the constant head boundary in 
semi-infinite ones [Rubin and Dagan, 1988, p. 1691]. In a bounded domain with two opposing constant head boundaries and no-flow conditions on the remaining sides, Smith and Freeze [1979b, pp. 1548-1550] found, via Monte Carlo simulations, that $\sigma_{h}^{2}$ depends on the location $\boldsymbol{x}, \sigma_{Y}^{2}$, the ratios $\lambda_{i} / L_{i}(i=1,2)$ of the integral scales to the lengths of the domain, and the form of the autocovariance of $\ln K$. A similar conclusion was reached analytically for semi-infinite domains by Dagan [1982a, pp. 825-826] and Rubin and Dagan [1988, p. 1691]. For three-dimensional infinite flow domains, in contrast to the one- and two-dimensional cases, there exists (under very general conditions) a stationary head solution to (2.12) [Gutjahr and Gelhar, 1981]; in particular, $\sigma_{h}^{2}$ is constant [Bakr et al., 1978; Dagan, 1979, 1982a]. However, for a three-dimensional bounded domain, Naff and Vecchia [1986, Figures 2-5] found that both the variance and the covariance of head exhibit non-stationary effects resulting from the imposition of no-flow boundary conditions.

Gelhar [1986, p. 140S] argued, based on physical grounds, that aquifer boundaries, although creating non-stationary head fields, produce effects limited only to their neighborhood. Thus, he advocated the use of the small perturbation and spectral approach, and consequently the assumption of locally stationary head variance, even for this class of problems. However, the quantitative determination of the boundary effects, for three-and two-dimensional flow domains, was accomplished only via a Green's function solution of (2.12) (small perturbation and Green's function approach) [Naff and Vecchia, 1986; Rubin and Dagan, 1988, 1989].

We recall here that Dagan [1989] imposes stationarity of head fluctuations by neglecting the divergence of a non constant term in the governing equation 2.40 for $\left\langle h^{(2)}\right\rangle$ (asymptotic expansion approach). This results in an incomplete expres- 
sion for $\boldsymbol{K}_{e}$. Development of the solution $h^{(1)}$ in $(2.50)$ by means of the Green's function method does not impose any condition on the head field since the method can equally well be applied to bounded domains. The Green's function approach is particularly appropriate for problems where conditioning on field-measured values is desired [Delhomme, 1979; Clifton and Neuman, 1982]. Here, even if the unconditional probability field is stationary, the ensuing conditional probability field is, in general, nonhomogeneous, i.e., the moments of the flow variables at a point $\boldsymbol{x}$ of the domain depend on the location of $\boldsymbol{x}$ relative to the sampling points [Dagan, 1982a]. Thus, the use of the Green's function approach (as done also in this thesis), in contrast to the spectral approach, allows the study of non-stationary head fields, arising from bounded flow domains and/or conditioning on field data.

\subsubsection{Asymptotic Expansion and Green's Function Approach}

Dagan [1989] assumed in equation 2.41 that $\boldsymbol{\alpha}=\left\langle Y^{\prime} \nabla h^{(1)}\right\rangle$ is constant, which implies that $\left\langle h^{(2)}\right\rangle$ satisfies Laplace's equation subject to homogeneous boundary conditions, which renders it zero. Consequently, ensemble averaging of $\boldsymbol{q}^{(2)}$ in (2.45) leads to $(2.46)$, where the term $-K_{G} \nabla\left\langle h^{(2)}\right\rangle$ has been eliminated from the expression for $\left\langle\boldsymbol{q}^{(2)}\right\rangle$. This term is of order $O\left(\sigma_{Y}^{2}\right)$. Dagan [1989, p. 204] studied the case of a bounded domain $\Omega$, consisting of a half-space with an impervious lower boundary $z=0$. He considered uniform mean flow with the mean hydraulic gradient vector $\boldsymbol{J}$ parallel to the impervious boundary. The zero order approximation of head was given by $h^{(0)}(\boldsymbol{x})=-\boldsymbol{J} \cdot \boldsymbol{x}$. Equation 2.50, with an appropriate Green's function $G$ for this bounded domain holds for the first order approximation $h^{(1)}(\boldsymbol{x})$. Such a Green's function can easily be obtained with the method of images [Stakgold, 1979]. On the 
impervious boundary $z=0, G$ satisfies $\partial G / \partial z=0$, and the same is true for $h^{(1)}(\boldsymbol{x})$ (and hence, $\alpha_{z}=0$ for $z=0$ ). Dagan [1989, p. 204] stated: "...As a result $\boldsymbol{\alpha}$, as well as $\boldsymbol{K}_{\text {ef }}$, is a function of the coordinate $z$ only, i.e. of the distance to the boundary...". He proceeded [ibid, p. 205] to evaluate $\alpha_{x}$ and $\alpha_{y}$, and consequently $\boldsymbol{K}_{\text {ef }}$, for $z=0$. However, since $\boldsymbol{\alpha}$ is a function of $z$, its divergence in (2.40) is not equal to zero, and hence, for this problem the use of $(2.42)$ is incorrect. Instead, $\left\langle h^{(2)}\right\rangle$ obeys a Poisson equation with homogeneous boundary conditions and so $\left\langle h^{(2)}\right\rangle \neq 0$. This means that the expression for $\boldsymbol{K}_{e}$ in (2.47) is incomplete and the term $-K_{G} \nabla\left\langle h^{(2)}\right\rangle=O\left(\sigma_{Y}^{2}\right)$ needs to be taken into account.

\subsection{Effective Conductivity for Infinite Domains}

The objective of this section is to show the need for some further approximations toward the analytical evaluation of (3.25), and to replicate expressions for $\boldsymbol{K}_{e}$ corresponding to three- and two- dimensional infinite flow domains under uniform mean hydraulic gradient by means of the residual flux theory.

We consider steady state flow in a three- or two- dimensional unbounded domain $\Omega_{\infty}$

$$
\nabla \cdot(K(\boldsymbol{x}) \nabla h(\boldsymbol{x}))+f(\boldsymbol{x})=0 \quad \text { for } \boldsymbol{x} \in \Omega_{\infty}
$$

where for the randomly prescribed source function $f(\boldsymbol{x})$ we set $\langle f(\boldsymbol{x})\rangle \equiv 0$. Flow is controlled by random boundary conditions at infinity which result in a uniform mean hydraulic gradient $\nabla\langle h(\boldsymbol{x})\rangle \equiv \boldsymbol{J}=$ constant. The same is true for $\nabla h_{\kappa}(\boldsymbol{x})$ and thus (3.25) reduces to

$$
\boldsymbol{r}(\boldsymbol{x}) \approx \boldsymbol{J} \int_{\Omega_{\infty}}\left\langle K^{\prime}(\boldsymbol{x}) K^{\prime}(\boldsymbol{\chi})\right\rangle \nabla_{x} \nabla_{\chi}^{T} G_{d}(\boldsymbol{\chi}, \boldsymbol{x}) d \boldsymbol{\chi}
$$


where the deterministic Green's function $G_{d}(\boldsymbol{\chi}, \boldsymbol{x})$ is defined via

$$
\nabla_{\chi} \cdot\left[\kappa \nabla_{\chi} G_{d}(\boldsymbol{\chi}, \boldsymbol{x})\right]+\delta(\boldsymbol{\chi}-\boldsymbol{x})=0 \quad \text { for } \boldsymbol{\chi}, \boldsymbol{x} \in \Omega_{\infty}
$$

Surface integrals do not appear because $G_{d}(\boldsymbol{\chi}, \boldsymbol{x})$ (in the three-dimensional case) and its gradient vanish at infinity.

The constant effective hydraulic conductivity tensor $\boldsymbol{K}_{e}$ is defined through (3.13) and (3.14),

$$
\langle\boldsymbol{q}\rangle=-\boldsymbol{K}_{e} \boldsymbol{J} \quad \boldsymbol{K}_{e}=\kappa \boldsymbol{I}-\tilde{\boldsymbol{\kappa}} .
$$

For $Y(\boldsymbol{x})=\ln K(\boldsymbol{x})$ and $Y^{\prime}(\boldsymbol{x})=Y(\boldsymbol{x})-\langle Y(\boldsymbol{x})\rangle$ Gaussian and statistically homogeneous, we have that $\kappa=\langle K(\boldsymbol{x})\rangle=K_{G} \exp \left[\sigma_{Y}^{2} / 2\right]$, the arithmetic mean of the hydraulic conductivities (Appendix A). The constant tensor $\tilde{\boldsymbol{\kappa}}$, defined in (3.12), is given by the integral term of (3.27),

$$
\tilde{\boldsymbol{\kappa}} \approx \int_{\Omega_{\infty}}\left\langle K^{\prime}(\boldsymbol{x}) K^{\prime}(\boldsymbol{\chi})\right\rangle \nabla_{x} \nabla_{\chi}^{T} G_{d}(\boldsymbol{x}, \boldsymbol{\chi}) d \boldsymbol{\chi}
$$

For $Y^{\prime}(\boldsymbol{x})$ Gaussian and statistically homogeneous, the following relationship holds (Appendix A),

$$
\left\langle K^{\prime}(\boldsymbol{x}) K^{\prime}(\boldsymbol{\chi})\right\rangle=K_{G}^{2} e^{\sigma_{Y}^{2}}\left[e^{\langle Y(\boldsymbol{x}) Y(\boldsymbol{\chi})\rangle}-1\right]
$$

The autocovariance of $Y(\boldsymbol{x}), C_{Y}(\boldsymbol{x}, \boldsymbol{\chi})=\langle Y(\boldsymbol{x}) Y(\boldsymbol{\chi})\rangle$, is given by the anisotropic (three- or two- dimensional) exponential function

$$
C_{Y}(\boldsymbol{\eta})=\sigma_{Y}^{2} \exp \left[-\left(\eta_{i}^{2} / \lambda_{i}^{2}\right)^{\frac{1}{2}}\right]
$$

where $\boldsymbol{\eta}=\boldsymbol{\chi}-\boldsymbol{x}, \lambda_{i}$ are principal integral scales, and the Einstein summation convention is utilized. Hereafter, norms of vectors are represented by unaccentuated versions of their boldface symbols. 
Using (3.30)-(3.31), and the transformation $G_{F}(\boldsymbol{\chi}, \boldsymbol{x})=K_{G} e^{\sigma_{Y}^{2} / 2} G_{d}(\boldsymbol{\chi}, \boldsymbol{x})$, the effective hydraulic conductivity in (3.29) becomes

$$
\boldsymbol{K}_{e}=K_{G} e^{\sigma_{Y}^{2} / 2}\left\{\boldsymbol{I}-\int_{\Omega_{\infty}}\left[e^{C_{Y}(\boldsymbol{\chi}-\boldsymbol{x})}-1\right] \nabla_{x} \nabla_{\chi}^{T} G_{F}(\boldsymbol{\chi}, \boldsymbol{x}) d \boldsymbol{\chi}\right\} .
$$

Here, $G_{F}(\boldsymbol{\chi}, \boldsymbol{x})$ satisfies the Poisson equation 3.28 ,

$$
\nabla^{2} G_{F}(\boldsymbol{\chi}, \boldsymbol{x})+\delta(\boldsymbol{\chi}-\boldsymbol{x})=0 \quad \text { for } \boldsymbol{x}, \boldsymbol{\chi} \in \Omega_{\infty}
$$

and constitutes a fundamental solution given in the three- and two- dimensional cases by $G_{F}(\boldsymbol{\chi}, \boldsymbol{x})=1 /(4 \pi \eta)$ and $G_{F}(\boldsymbol{\chi}, \boldsymbol{x})=1 / 2 \pi \ln \eta$, respectively [Greenberg, 1971, 1978].

Expression 3.33 is evaluated for the three-dimensional case in Appendix $\mathrm{F}$ by expanding the term $e^{C_{Y}(\boldsymbol{\chi}-\boldsymbol{x})}-1$ in Taylor series and performing the integration in Fourier space following the methodology of Neuman et al. [1987] (see also Zhang [1990]). For the statistically isotropic case we obtain (Appendix F)

$$
K_{e}=\frac{K_{G}}{3} e^{\frac{\sigma_{Y}^{2}}{2}}\left(4-e^{\sigma_{Y}^{2}}\right),
$$

which results in negative $K_{e}$ for $\sigma_{Y}^{2} \geq 1.386$. This physically impossible situation results from the use of different order approximations for the stochastic Green's function (zeroth order approximation) and the autocovariance (exact evaluation).

We proceed instead, here and in the rest of this dissertation, to approximate all functions in (3.33) to first order in $\sigma_{Y}^{2}$. For infinite domains this amounts to writing (3.33) as

$$
\boldsymbol{K}_{e}=K_{G}\left\{\left(1+\frac{\sigma_{Y}^{2}}{2}\right) \boldsymbol{I}-\int_{\Omega_{\infty}} C_{Y}(\boldsymbol{\chi}-\boldsymbol{x}) \nabla_{x} \nabla_{\chi}^{T} G_{F}(\boldsymbol{\chi}, \boldsymbol{x}) d \boldsymbol{\chi}\right\} .
$$

Equation 3.36 is identical to the linearized expression for $\boldsymbol{K}_{e}$, equations 2.47 and 2.51, of Dagan [1989]. The analysis in Appendix F, for three-dimensional infinite 
domains, can still be utilized for the derivation of $\boldsymbol{K}_{e}$ as given in (3.36) by retaining now only the first term $(m=1)$ of the infinite series for the exponential, and first order approximation in $\sigma_{Y}^{2}$ of all expressions. Thus, equation F.10 becomes

$$
K_{e_{i j}}=K_{G}\left\{\left(1+\frac{\sigma_{Y}^{2}}{2}\right)-\frac{1}{(2 \pi)^{\frac{3}{2}}} \int_{\boldsymbol{k}} \frac{\boldsymbol{k} \boldsymbol{k}^{T}}{k^{2}} \Phi_{I}(\boldsymbol{k}) d \boldsymbol{k}\right\},
$$

where $i, j=1,2,3$, and $K_{e_{i j}}=0$ for $i \neq j$. The spectrum of the three-dimensional anisotropic exponential covariance function, equation F.9, is given now by

$$
\Phi_{I}(\boldsymbol{k})=\Phi_{I}(-\boldsymbol{k})=\sqrt{\frac{8}{\pi}} \frac{\sigma_{Y}^{2} \lambda_{1} \lambda_{2} \lambda_{3}}{\left[1+\left(\lambda_{i} k_{i}\right)^{2}\right]^{2}},
$$

where summation over the index $i=1,2,3$ is implied. We can write

$$
k^{2}=\left\|k^{2}\right\|=\boldsymbol{k}^{T} \boldsymbol{k} \quad ; \quad \boldsymbol{k} \boldsymbol{k}^{T}=k_{i} k_{j}
$$

where $i, j=1,2,3$, and $k_{i}$ are components of the wave-number vector $\boldsymbol{k}$. Equation 3.37 has appeared frequently in the stochastic literature. Thus, (3.37) corresponds exactly to the three-dimensional expression for $\boldsymbol{K}_{e}$ in (2.23) of Gutjahr et al. [1978] and Gelhar and Axness [1983a], equations 13 and 14 of Neuman and Depner [1988], (2.47) and (2.52) of Dagan [1989], and equation 81 of Kitanidis [1990]. Different constants in front of the integral term arise merely from different definitions of the Fourier transform (FT) pair [Bakr et al., 1978, equation 23; Gutjahr et al., 1978; Gelhar and Axness, 1981, equations (A3) and (A4); Gelhar and Axness, 1983a; Neuman and Depner, 1988].

Different methods have been used by the above researchers to evaluate the integral term in (3.36) or (3.37). For one-dimensional flow, Gutjahr et al. [1978] observed that the integral term equals, by definition, $\sigma_{Y}^{2}$; for statistically isotropic media in two- and three-dimensional flow domains each of these terms equals $\sigma_{Y}^{2} / E$ 
(page 27). Gelhar and Axness [1981, 1983a] for an exponential anisotropic covariance, Neuman and Depner [1988] and Dagan [1989] for an arbitrary anisotropic covariance, defined a coordinate system that renders the autocovariance isotropic and subsequently transformed (3.37) into spherical coordinates (a similar approach for the two-dimensional case is used in Appendix E). Gelhar and Axness [1981, Appendix G], and Dagan [1989, pp. 191, 192] succeeded in reducing the triple integral to a single and double integral, respectively. Neuman and Depner [1988, Appendix B] and Dagan [1989, p. 192] have shown that (3.37) is independent of the spectral density $\Phi_{I}(\boldsymbol{k})$ but depends only on $\sigma_{Y}^{2}$ and the ratios between the integral scales. Dykaar and Kitanidis [1992b] evaluated numerically the discrete form of (3.37), i.e., they represented the multiple integral in (3.37) by a multiple summation.

Equation F.19 becomes, to first order in $\sigma_{Y}^{2}$,

$$
K_{e_{i i}}=K_{G}\left\{\left(1+\frac{\sigma_{Y}^{2}}{2}\right)-\frac{\sigma_{Y}^{2}}{4 \pi \zeta_{1} \zeta_{2} \zeta_{3}} \int_{u=-1}^{+1} \int_{\psi=0}^{2 \pi} \frac{\boldsymbol{G}_{i i}}{B^{3}} d u d \psi\right\},
$$

where $G, B$, and $\zeta_{i}$ are defined in (F.14)-(F.16) and $i=1,2,3$. Equation 3.40 corresponds to equations 5.8, G8, G10, and G11 of Gelhar and Axness [1981], equation 52 of Gelhar and Axness [1983a], and equations 3.4.16 and 3.4.14 of Dagan [1989].

For the statistically isotropic case, substituting (F.21) into (3.40) gives

$$
K_{e}=K_{G}\left\{1+\frac{\sigma_{Y}^{2}}{6}\right\}
$$

which has previously been derived by Gutjahr et al. [1978], Gelhar and Axness [1981, 1983a], Dagan [1982a, 1989], and Kitanidis [1990].

To obtain expressions valid for large $\sigma_{Y}^{2}$, we adopt in the remaining of the dissertation a conjecture similar to that proposed for isotropic fields by Landau and Lifshitz [1960], Shvidler [1962], and Matheron [1967], and utilized later for anisotropic 
fields by Gelhar and Axness [1983a] and Neuman and Depner [1988], that the expressions within braces in (3.40) and (3.41) constitute the first two terms in a series expansion of an exponential.

The linearized expression 3.41, its exponential generalization, equation 2.27 $(E=3)$, and the numerical solution by Levin [1992], are compared in Figure 3.1 for various $\sigma_{Y}^{2}$ (also in Neuman et al. [1992]). The numerical solution was obtained via high-resolution Monte Carlo simulations of uniform mean flow across a finite element grid of $35 \times 35 \times 35$ trilinear cubes in a statistically homogeneous, isotropic, lognormal $K(\boldsymbol{x})$ field with an exponential covariance. Random realizations of $K(\boldsymbol{x})$ were generated with the turning band method of Tompson et al. [1989] so as to obtain an integral scale of five cube lengths. Point values of $K(\boldsymbol{x})$, generated at the centroid of the cubes, were assigned without change to the corresponding cubes. The finite element flow equations were then solved in residual form by preconditioned conjugate gradients (with incomplete LU factorization) using the ESSL-IBM package DSMGCG. Constant heads were imposed on two opposing sides of the cubic flow domain, and-no flow conditions were imposed on the remaining sides. The global mass balance error, averaged over 500 simulations, increased from $0.002 \%$ for $\sigma_{Y}^{2}=$ 0.25 to $0.5 \%$ for $\sigma_{Y}^{2}=7$. The largest global mass balance error during any simulation was less than $3 \%$.

Figure 3.1 shows an excellent agreement between the exponential solution and the numerical results up to at least $\sigma_{Y}^{2}=7$. A similar agreement with Monte Carlo results, generated with a different numerical method, has been reported recently by Dykaar and Kitanidis [1992b], Figure 2.2, for $\sigma_{Y}^{2}$ as large as 6 , and by Desbarats [1992a] for $\sigma_{Y}^{2} \leq 3$ (25 realizations); Ababou [1988] found earlier, on the 
basis of a single realization in a large domain, that this agreement holds at least up to $\sigma_{Y}^{2}=5.3$. This may explain why, when (3.37) was applied by Neuman and Depner [1988] to data obtained from relatively small-scale single-hole packer tests in fractured granites, according to which $\sigma_{Y}^{2}>7$ and $Y(\boldsymbol{x})$ is statistically anisotropic, it showed consistency with the results of much larger-scale cross-hole tests conducted and interpreted independently of the single-hole tests.

The residual flux theory, at the level of approximation performed in (3.25) and the consistent approximation of other terms, replicates (Appendix $\mathrm{J}$ ) the closed form expressions 2.33 and 2.35, derived in Chapter 2 for two-dimensional anisotropic media from expressions due to Gelhar and Axness [1983a]. A similar conclusion was reached by Sanchez-Villa and Carrera [1993], Appendix J, using the asymptotic expansion approach of Dagan [1989]. All three results yield (2.72) which corresponds to Ababou's conjecture [1991].

In conclusion, for infinite domains and anisotropic or isotropic media, the small perturbation and spectral approach, the asymptotic expansion and Green's function approach, and the zeroth order approximation of the residual flux theory produce identical results, which is a natural consequence of their common starting point, equation 3.36 or 3.37 . However, the residual flux theory can address bounded flow domains as demonstrated in the following chapters. 


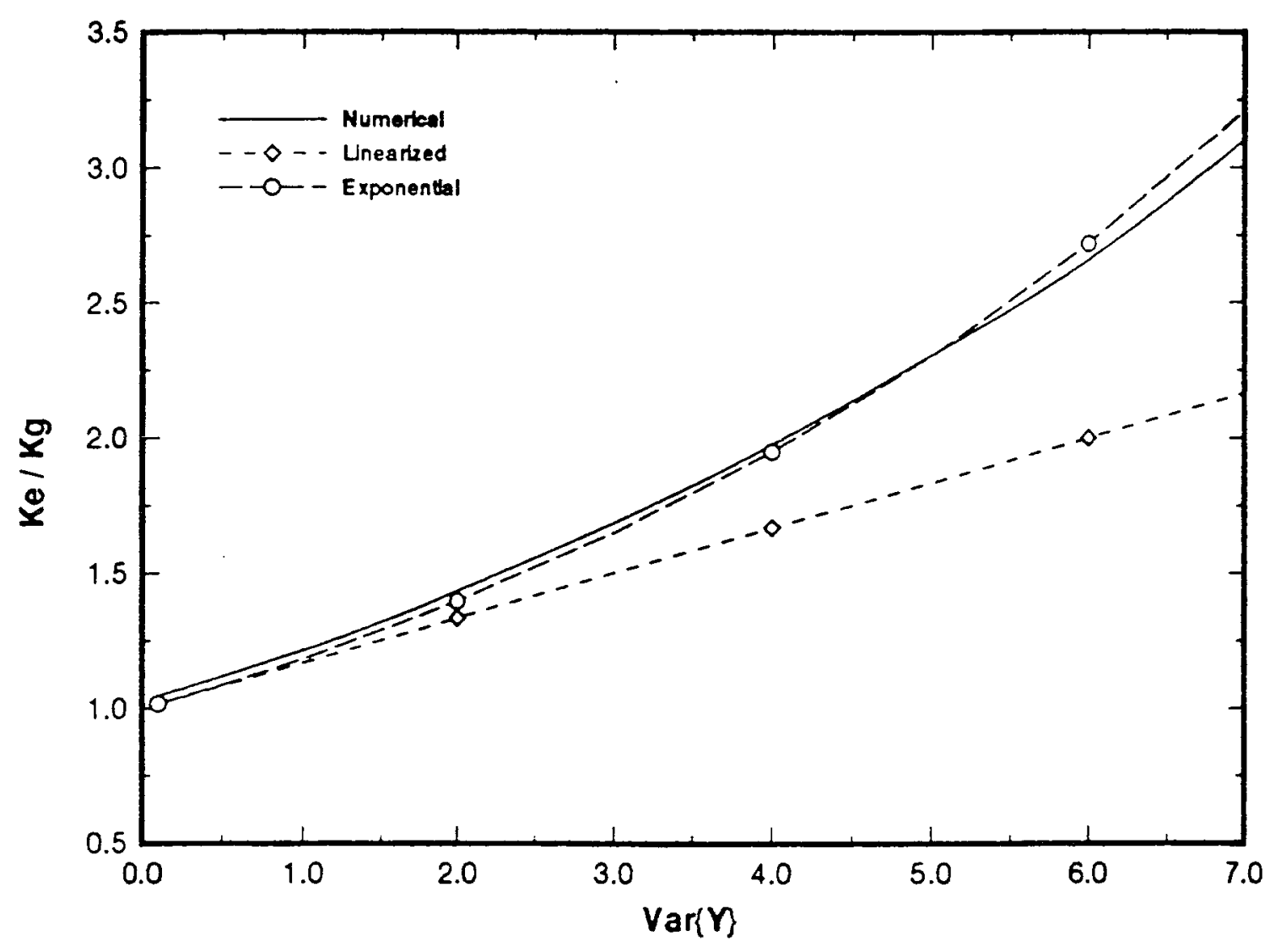

Figure 3.1: Comparison between $(2.26),(2.27)$ and numerical $K_{e}$. 


\section{CHAPTER 4}

\section{EFFECTIVE CONDUCTIVITY FOR 3-D BOUNDED DOMAINS}

\subsection{Basic Equation for the Effective Hydraulic Conductivity}

We consider a flow domain $\Omega$, Figure 4.1, consisting of two planes that extend infinitely in the $\chi_{2^{-}}$and $\chi_{3^{-}}$-directions, and are separated by a distance $2 a$ in the $\chi_{1}$-direction. On the boundary $\Gamma_{D 1}^{-}$traversed by the negative $\chi_{1}$-axis, $\langle H(\chi)\rangle=$ $H_{2}=$ constant , and on the opposite boundary $\Gamma_{D 1}^{+}$traversed by the positive $\chi_{1^{-}}$axis, $\langle H(\boldsymbol{\chi})\rangle=H_{1}=$ constant. At infinity $\langle Q(\boldsymbol{\chi})\rangle=0$, and in the interior of the domain, $\langle f(\boldsymbol{x})\rangle=0$.

Then, in (3.25) the integral on the $\Gamma_{N}$ boundary vanishes, and the integral on $\Gamma_{D}$ becomes

$$
\begin{aligned}
& \int_{\Gamma_{D}}\left\langle K^{\prime}(\boldsymbol{x}) K^{\prime}(\boldsymbol{\chi})\right\rangle \nabla_{x} \nabla_{\chi}^{T} G_{d}(\boldsymbol{x}, \boldsymbol{\chi}) \boldsymbol{n}(\boldsymbol{\chi})\langle H(\boldsymbol{\chi})\rangle d \boldsymbol{\chi} \\
= & \int_{\Gamma_{D 1}^{+}}\left\langle K^{\prime}(\boldsymbol{x}) K^{\prime}(\boldsymbol{\chi})\right\rangle \nabla_{x} \nabla_{\chi}^{T} G_{d}(\boldsymbol{x}, \boldsymbol{\chi}) \boldsymbol{n}^{*}(\boldsymbol{\chi}) H_{1} d \boldsymbol{\chi} \\
+ & \int_{\Gamma_{D 1}^{-}}\left\langle K^{\prime}(\boldsymbol{x}) K^{\prime}(\boldsymbol{\chi})\right\rangle \nabla_{x} \nabla_{\chi}^{T} G_{d}(\boldsymbol{x}, \boldsymbol{\chi})\left(-\boldsymbol{n}^{*}(\boldsymbol{\chi})\right) H_{2} d \boldsymbol{\chi} \\
= & \left(H_{1}-H_{2}\right) \int_{\Gamma_{D 1}^{+}}\left\langle K^{\prime}(\boldsymbol{x}) K^{\prime}(\boldsymbol{\chi})\right\rangle \nabla_{x} \nabla_{\chi}^{T} G_{d}(\boldsymbol{x}, \boldsymbol{\chi}) \boldsymbol{n}^{*}(\boldsymbol{\chi}) d \boldsymbol{\chi},
\end{aligned}
$$

where $G_{d}(\boldsymbol{x}, \boldsymbol{\chi})$ is the deterministic Green's function defined through (3.22)-(3.24), $K^{\prime}(\boldsymbol{x})$ is the perturbation of the hydraulic conductivity $K(\boldsymbol{x})$, and $\boldsymbol{n}^{*}(\boldsymbol{\chi})$ is a unit vector normal to the $\Gamma_{D 1}^{+}$boundary. 


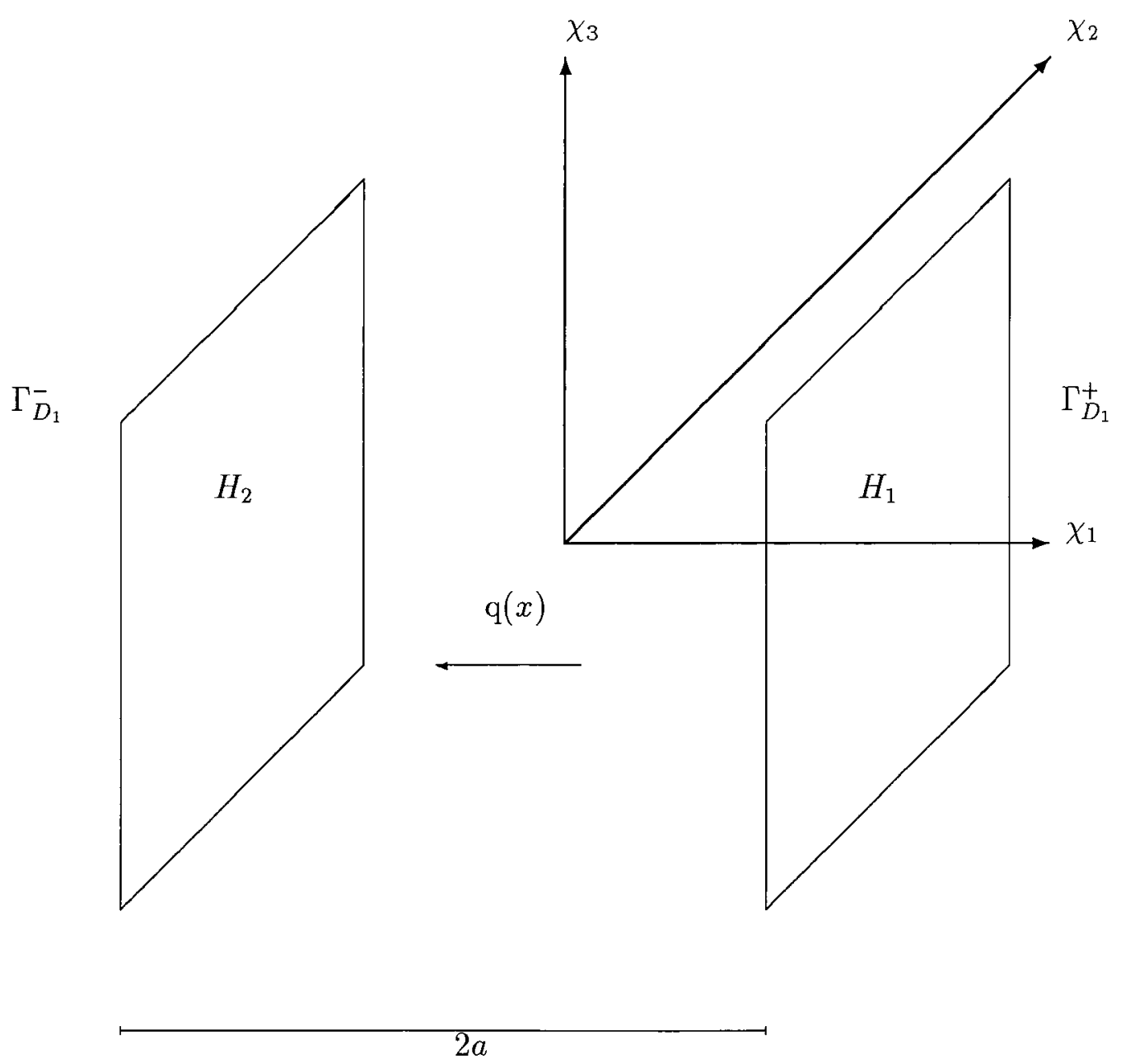

Figure 4.1: The flow domain $\Omega$. 
Substituting (4.1) into (3.25) the latter becomes

$$
\begin{aligned}
\boldsymbol{r}(\boldsymbol{x}) & \approx \int_{\Omega}\left\langle K^{\prime}(\boldsymbol{x}) K^{\prime}(\boldsymbol{\chi})\right\rangle \nabla_{x} \nabla_{\chi}^{T} G_{d}(\boldsymbol{x}, \boldsymbol{\chi}) \nabla_{\chi} h_{k}(\boldsymbol{\chi}) d \boldsymbol{\chi} \\
& +\left(H_{2}-H_{1}\right) \int_{\Gamma_{D 1}^{+}}\left\langle K^{\prime}(\boldsymbol{x}) K^{\prime}(\boldsymbol{\chi})\right\rangle \nabla_{x} \nabla_{\chi}^{T} G_{d}(\boldsymbol{x}, \boldsymbol{\chi}) \boldsymbol{n}^{*}(\boldsymbol{\chi}) d \boldsymbol{\chi}
\end{aligned}
$$

We designate the gradient of $h_{k}(\boldsymbol{\chi})$ by $\boldsymbol{J}$,

$$
\boldsymbol{J}=\left(\frac{H_{1}-H_{2}}{2 a}, 0,0\right)^{T},
$$

where $2 a$ is the distance between the Dirichlet boundaries. Substituting (4.3) into (4.2) gives

$$
\begin{aligned}
\boldsymbol{r}(\boldsymbol{x}) \approx & \left\{\int_{\Omega}\left\langle K^{\prime}(\boldsymbol{x}) K^{\prime}(\boldsymbol{\chi})\right\rangle \nabla_{x} \nabla_{\chi}^{T} G_{d}(\boldsymbol{x}, \boldsymbol{\chi}) d \boldsymbol{\chi}\right. \\
& \left.-2 a \int_{\Gamma_{D 1}^{+}}\left\langle K^{\prime}(\boldsymbol{x}) K^{\prime}(\boldsymbol{\chi})\right\rangle \nabla_{x} \nabla_{\chi}^{T} G_{d}(\boldsymbol{x}, \boldsymbol{\chi}) d \boldsymbol{\chi}\right\} \boldsymbol{J}
\end{aligned}
$$

where the term in braces is a symmetric second-rank tensor $\tilde{\boldsymbol{\kappa}}(\boldsymbol{x})$. Thus, the residual flux can be expressed as

$$
\boldsymbol{r}(\boldsymbol{x}) \approx \tilde{\boldsymbol{\kappa}}(\boldsymbol{x}) \boldsymbol{J}
$$

Equations (4.4) and (4.5) hold because the working coordinates $\boldsymbol{x}$ are oriented parallel to the principal directions of statistical anisotropy of $K^{\prime}$, and then symmetry implies that $\nabla\langle h(\boldsymbol{x})\rangle \equiv \nabla h_{k}(\boldsymbol{x}) \equiv \boldsymbol{J}$. Since stationarity prevails on planes parallel to the constant head boundaries with $\left\langle q_{x_{2}}\right\rangle=\left\langle q_{x_{3}}\right\rangle=0$ (from Figure 4.1 the mean hydraulic gradient exists only in the $x_{1}$-direction), and by virtue of the continuity equation (3.8) with $\langle f(\boldsymbol{x})\rangle=0$, we have $d\left\langle q_{x_{1}}\right\rangle / d x_{1}=0$. Hence $\left\langle q_{x_{1}}\right\rangle$ is constant in the $x_{1}$-direction which implies that $\boldsymbol{r}(\boldsymbol{x})$ remains constant in the direction of the mean flow. Then the residual flux has only one nonzero component parallel to $x_{1}$,

$$
r_{1}\left(x_{2}, x_{3}\right) \approx \tilde{\kappa}_{1}\left(x_{2}, x_{3}\right) J_{1},
$$


where

$$
\begin{aligned}
\tilde{\kappa}_{1}\left(x_{2}, x_{3}\right)= & \int_{\Omega}\left\langle K^{\prime}(\boldsymbol{x}) K^{\prime}(\boldsymbol{\chi})\right\rangle \frac{\partial^{2}}{\partial x_{1} \partial \chi_{1}} G_{d}(\boldsymbol{x}, \boldsymbol{\chi}) d \boldsymbol{\chi}- \\
& 2 a \int_{\Gamma_{D 1}^{+}}\left\langle K^{\prime}(\boldsymbol{x}) K^{\prime}(\boldsymbol{\chi})\right\rangle \frac{\partial^{2}}{\partial x_{1} \partial \chi_{1}} G_{d}(\boldsymbol{x}, \boldsymbol{\chi}) d \boldsymbol{\chi}
\end{aligned}
$$

Using (A.9),

$$
\left\langle K^{\prime}(\boldsymbol{x}) K^{\prime}(\boldsymbol{\chi})\right\rangle=K_{G}^{2} e^{\sigma_{Y}^{2}}\left[e^{\langle Y(\boldsymbol{x}) Y(\chi)\rangle}-1\right]
$$

and the transformation $G_{F}(\boldsymbol{x}, \boldsymbol{\chi})=K_{G} \exp \left(\sigma_{Y}^{2} / 2\right) G_{d}(\boldsymbol{x}, \boldsymbol{\chi})$, equation (4.7) becomes

$$
\begin{aligned}
\tilde{\kappa}_{1}\left(x_{2}, x_{3}\right)= & K_{G} e^{\sigma_{Y}^{2} / 2}\left\{\int_{\Omega}\left[e^{\langle Y(\boldsymbol{x}) Y(\boldsymbol{\chi})\rangle}-1\right] \frac{\partial^{2}}{\partial x_{1} \partial \chi_{1}} G_{F}(\boldsymbol{x}, \boldsymbol{\chi}) d \boldsymbol{\chi}-\right. \\
& \left.2 a \int_{\Gamma_{D 1}^{+}}\left[e^{\langle Y(\boldsymbol{x}) Y(\boldsymbol{\chi})\rangle}-1\right] \frac{\partial^{2}}{\partial x_{1} \partial \chi_{1}} G_{F}(\boldsymbol{x}, \boldsymbol{\chi}) d \boldsymbol{\chi}\right\}
\end{aligned}
$$

Here, $G_{F}(\boldsymbol{x}, \boldsymbol{\chi})$ satisfies (equations 3.22 to 3.24 )

$$
\nabla_{\chi}^{2} G_{F}(\boldsymbol{x}, \boldsymbol{\chi})+\delta(\boldsymbol{\chi}-\boldsymbol{x})=0 \quad \text { for } \boldsymbol{x}, \boldsymbol{\chi} \in \Omega
$$

with boundary conditions

$$
\begin{aligned}
& G_{F}\left(-a, \chi_{2}, \chi_{3}, \boldsymbol{x}\right)=0, \\
& G_{F}\left(+a, \chi_{2}, \chi_{3}, \boldsymbol{x}\right)=0 .
\end{aligned}
$$

The derivatives of $G_{F}$ with respect to $\chi_{2}$ and $\chi_{3}$ vanish far from the origin. Using the method of images [Stakgold, 1979] as in Figure 4.2 one obtains

$$
G_{F}(\boldsymbol{x}, \boldsymbol{\chi})=+\frac{1}{4 \pi} \sum_{j=-\infty}^{+\infty} \frac{(-1)^{j}}{\left[\left(\chi_{1}-2 j a-(-1)^{j} x_{1}\right)^{2}+\left(\chi_{2}-x_{2}\right)^{2}+\left(\chi_{3}-x_{3}\right)^{2}\right]^{1 / 2}}
$$

When $a \rightarrow \infty$, all terms except the one corresponding to $j=0$ vanish and one recovers the three-dimensional fundamental solution [Greenberg, 1971]. 


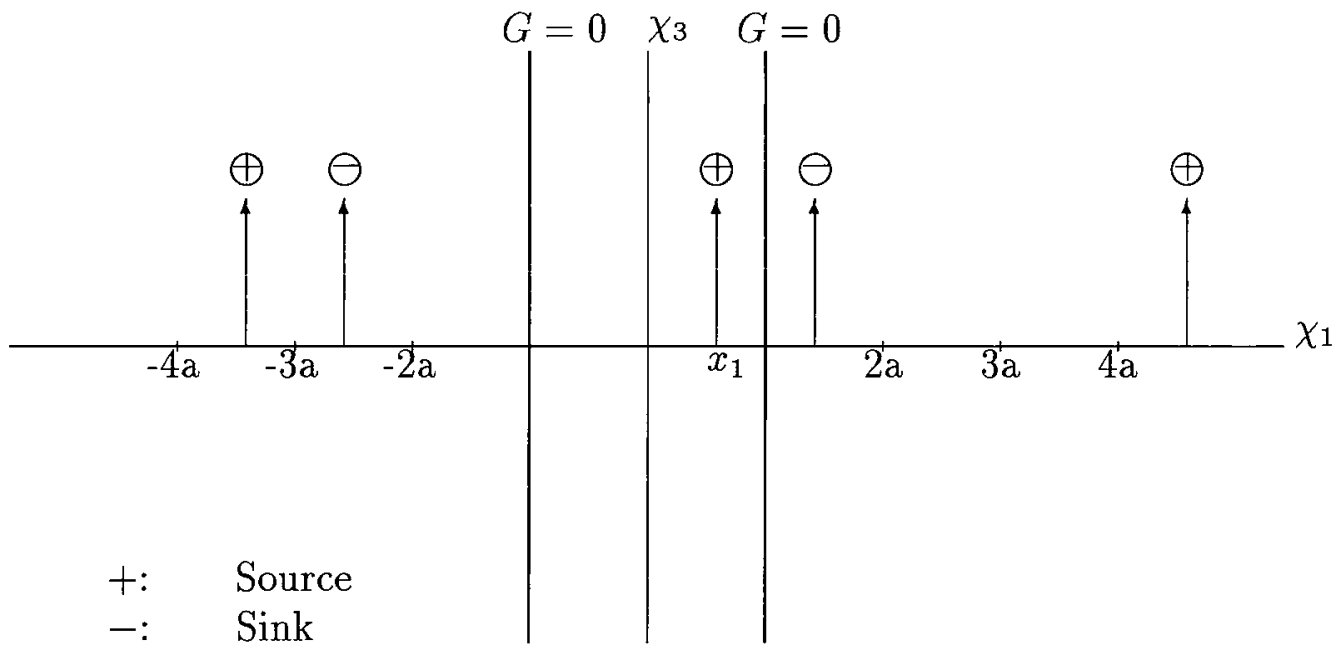

Figure 4.2: Green's function's infinite series of images. 
Approximating all functions in (4.9) to first order and substituting into (3.14) yields, for the effective hydraulic conductivity,

$$
\begin{aligned}
K_{e}= & K_{G}\left\{\left(1+\frac{\sigma_{Y}^{2}}{2}\right)-\int_{\Omega} C_{Y}(\boldsymbol{\chi}-\boldsymbol{x}) \frac{\partial^{2}}{\partial x_{1} \partial \chi_{1}} G_{F}(\boldsymbol{x}, \boldsymbol{\chi}) d \boldsymbol{\chi}\right. \\
& \left.+2 a \int_{\Gamma_{D 1}^{+}} C_{Y}(\boldsymbol{\chi}-\boldsymbol{x}) \frac{\partial^{2}}{\partial x_{1} \partial \chi_{1}} G_{F}(\boldsymbol{x}, \boldsymbol{\chi}) d \boldsymbol{\chi}\right\}
\end{aligned}
$$

Here, $C_{Y}(\boldsymbol{x}-\boldsymbol{x})$ is the autocovariance of $Y$, and $\kappa(\boldsymbol{x})=\kappa=K_{G} \exp \left[\sigma_{Y}^{2} / 2\right]$ in $(3.14)$ was linearized to first order in $\sigma_{Y}^{2}$. On the left hand side of (4.14) the dependence of $K_{e}$ on $x_{2}$ and $x_{3}$ has been dropped, for reasons to become apparent later in the analysis.

We set

$$
\mathcal{H}=\int_{\chi_{2}=-\infty}^{+\infty} \int_{\chi_{3}=-\infty}^{+\infty} C_{Y}(\boldsymbol{\eta}) \frac{\partial^{2}}{\partial x_{1} \partial \chi_{1}} G_{F}(\boldsymbol{x}, \boldsymbol{\chi}) d \chi_{2} d \chi_{3}
$$

with

$$
\boldsymbol{\eta}=\boldsymbol{\chi}-\boldsymbol{x} \quad ; \quad \boldsymbol{\chi}=\left(\chi_{1}, \chi_{2}, \chi_{3}\right)^{T} \quad, \quad \boldsymbol{x}=\left(x_{1}, x_{2}, x_{3}\right)^{T}
$$

Then the third term within braces in (4.14), which incorporates the surface effects, is

$$
S=-2 a \mathcal{H}
$$

where $S$ must be evaluated at $\chi_{1}=+a$. Likewise, the domain integral over $\Omega$ is

$$
D=\int_{\chi_{1}=-a}^{\chi_{1}=+a} \mathcal{H} d \chi_{1}
$$

so that $K_{e}$ in $(4.14)$ becomes

$$
K_{e}=K_{G}\left\{1+\frac{\sigma_{Y}^{2}}{2}-(D+S)\right\}
$$


Adopting the Landau-Lifshitz [1960] conjecture, an expression for $K_{e}$ valid for large $\sigma_{Y}^{2}$ is given by

$$
K_{e}=K_{G} \exp \left\{\frac{\sigma_{Y}^{2}}{2}-(D+S)\right\}
$$

We define

$$
\begin{gathered}
\Lambda\left(-k_{2},-k_{3} ; \chi_{1}, \boldsymbol{x}\right)=\frac{1}{2 \pi} \int_{-\infty}^{+\infty} \int_{-\infty}^{+\infty} C_{Y}\left(\boldsymbol{\chi}^{\prime} ; \chi_{1}, \boldsymbol{x}\right) e^{-i \boldsymbol{k}^{\prime} \cdot \boldsymbol{\chi}^{\prime}} d \boldsymbol{\chi}^{\prime} \\
F\left(k_{2}, k_{3} ; \chi_{1}, \boldsymbol{x}, a\right)=\frac{1}{2 \pi} \int_{-\infty}^{+\infty} \int_{-\infty}^{+\infty} \frac{\partial^{2}}{\partial x_{1} \partial \chi_{1}} G_{F}\left(\boldsymbol{\chi}^{\prime} ; \chi_{1}, \boldsymbol{x}, a\right) e^{i \boldsymbol{k}^{\prime} \cdot \boldsymbol{\chi}^{\prime}} d \boldsymbol{\chi}^{\prime}
\end{gathered}
$$

with

$$
\boldsymbol{k}^{\prime}=\left(k_{2}, k_{3}\right)^{T} \quad ; \quad \chi^{\prime}=\left(\chi_{2}, \chi_{3}\right)^{T}
$$

$\boldsymbol{k}=\left(k_{1}, k_{2}, k_{3}\right)^{T}$ denoting the three-dimensional wave number vector. Under the transformation

$$
\boldsymbol{\eta}^{\prime}=\boldsymbol{\chi}^{\prime}-\boldsymbol{x}^{\prime} \quad ; \quad \boldsymbol{x}^{\prime}=\left(x_{2}, x_{3}\right)^{T}
$$

(4.21) and (4.22) become

$$
\begin{gathered}
\Lambda\left(-k_{2},-k_{3} ; \chi_{1}, \boldsymbol{x}\right)= \\
\frac{1}{2 \pi} e^{-i \boldsymbol{k}^{\prime} \cdot \boldsymbol{x}^{\prime}} \int_{-\infty}^{+\infty} \int_{-\infty}^{+\infty} C_{Y}\left(\boldsymbol{\eta}^{\prime} ; \chi_{1}-x_{1}\right) e^{-i \boldsymbol{k}^{\prime} \cdot \boldsymbol{\eta}^{\prime}} d \boldsymbol{\eta}^{\prime}, \\
F\left(k_{2}, k_{3} ; \chi_{1}, \boldsymbol{x}, a\right)= \\
\frac{1}{2 \pi} e^{+i \boldsymbol{k}^{\prime} \cdot \boldsymbol{x}^{\prime}} \int_{-\infty}^{+\infty} \int_{-\infty}^{+\infty} \frac{\partial^{2}}{\partial x_{1} \partial \chi_{1}} G_{F}\left(\boldsymbol{\eta}^{\prime} ; \chi_{1}, x_{1}, a\right) e^{i \boldsymbol{k}^{\prime} \cdot \boldsymbol{\eta}^{\prime}} d \boldsymbol{\eta}^{\prime} .
\end{gathered}
$$

With the aid of Parseval's identity (B.7), the integral $\mathcal{H}$ is transformed via (4.25) and (4.26) into the Fourier space integral

$$
\mathcal{H}\left(\chi_{1}, x_{1}, a\right)=\int_{\boldsymbol{k}^{\prime}} \Lambda F d \boldsymbol{k}^{\prime}
$$


Since $S$ is given by evaluating $\mathcal{H}\left(\chi_{1}, x_{1}, a\right)$ at $\chi_{1}=a$ we have that $S=S\left(x_{1}, a\right)$. Similarly, $D=D\left(x_{1}, a\right)$, and hence $K_{e}$ in (4.19) or (4.20) is also a function of $x_{1}$ and $a$. However, we made clear earlier (page 71) that $\left\langle q_{x_{1}}\right\rangle$ is constant in the $x_{1}$-direction, hence the same is true for the effective conductivity, i.e., $K_{e}=K_{e}(a)$.

\subsection{Two-Dimensional FT of the Covariance Function}

To evaluate the effective hydraulic conductivity in (4.14) we need to assume a functional form for $C_{Y}(\boldsymbol{\chi}-\boldsymbol{x})$, the autocovariance of $Y$. For infinite flow domains, under a uniform mean gradient in normally distributed and statistically homogeneous $\ln K$ fields, $K_{e}$ has been shown to be independent of $C_{Y}(\boldsymbol{\chi}-\boldsymbol{x})$. Matheron [1967] has shown that, for statistically isotropic $\ln K$ fields (see also Gelhar [1986] for two-dimensional media, and Gutjahr et al. [1978] for one-, two-, and three-dimensional domains), $K_{e}$ depends only on the variance of $Y(\boldsymbol{x})$. This was verified numerically (Figure 2.1) for exponential and Gaussian isotropic functions by Dykaar and Kitanidis [1992b]. Neuman and Depner [1988] and Dagan [1989] found that, in the presence of ellipsoidal or geometric statistical anisotropy (examples include exponential, Gaussian, and spherical covariance functions), the solution depends additionally on the ratios between principal correlation scales, but not on their values.

However, for bounded flow domains, numerical studies have indicated that the form of the covariance function may have an effect on $K_{e}$. Dykaar and Kitanidis [1992b] found that, for statistically isotropic media with "small $\sigma_{Y}^{2}$ " (a value for the variance is not provided), the three- (two-) dimensional infinite domain solution is attained for a cube (square) with characteristic length of 10 (20) integral scales. Figure 2.1 [Dykaar and Kitanidis, 1992b] indicates that, for smaller flow domains the input 
covariance function influences $K_{e}$. Figure 2.3 [Fenton and Griffiths, 1993] shows that the values of the normalized sample means of block conductivity $\bar{K}$, for aspect ratios $X_{L} / Y_{L}=1 / 9,9 / 1$ of horizontal to vertical length of a rectangular domain, differ for three isotropic exponential correlation functions. In both studies the small difference in the solutions for different input covariances may be the result of numerical error. However, it seems reasonable in view of (4.14) to expect an influence on $K_{e}$ from the input covariance as different covariance functions are truncated differently by the boundaries.

In this study we will employ an anisotropic exponential function, (3.32), to describe the spatial correlation structure of $Y$. The exponential and the spherical covariances constitute the most popular models in the representation of field data [Delhomme, 1979; Clifton, 1981; Hoeksema and Kitanidis, 1985; Sudicky, 1986; Neuman and Depner, 1988; Gómez-Hernández and Gorelick, 1989; Desbarats and Dimitrakopoulos, 1990]. However, the exponential due to its simplicity is particularly advantageous for analytical studies [Bakr et al., 1978; Gelhar and Axness, 1981, 1983a; Dagan, 1982a, b; Rubin and Dagan, 1988, 1989; Rubin and Gómez-Hernández, 1990] and as such it will be used in this dissertation.

Define

$$
I_{1}=\frac{1}{2 \pi} \int_{-\infty}^{+\infty} \int_{-\infty}^{+\infty} C_{Y}\left(\boldsymbol{\eta}^{\prime} ; \chi_{1}-x_{1}\right) e^{-i \boldsymbol{k}^{\prime} \cdot \boldsymbol{\eta}^{\prime}} d \boldsymbol{\eta}^{\prime}
$$

and the transformations

$$
\begin{aligned}
& \chi_{2}^{*}=\frac{\eta_{2}}{\lambda_{2}}=\frac{\chi_{2}-x_{2}}{\lambda_{2}}, \\
& \chi_{3}^{*}=\frac{\eta_{3}}{\lambda_{3}}=\frac{\chi_{3}-x_{3}}{\lambda_{3}},
\end{aligned}
$$


where

$$
\chi^{*^{2}}=\left\|\chi^{*}\right\|^{2}=\frac{\eta_{2}^{2}}{\lambda_{2}^{2}}+\frac{\eta_{3}^{2}}{\lambda_{3}^{2}}
$$

Upon setting

$$
\gamma=\frac{\left|\eta_{1}\right|}{\lambda_{1}}=\frac{\left|\chi_{1}-x_{1}\right|}{\lambda_{1}}
$$

the exponential covariance function in (3.32) becomes isotropic,

$$
C_{Y}\left(\chi^{*} ; \gamma\right)=\sigma_{Y}^{2} e^{-\sqrt{\gamma^{2}+\chi^{*}}}
$$

Let us also define a vector

$$
\boldsymbol{k}^{*}=\boldsymbol{L}^{\prime} \boldsymbol{k}^{\prime}
$$

where $\boldsymbol{L}^{\prime}$ is a $2 \times 2$ diagonal matrix with elements $\lambda_{2}$ and $\lambda_{3}$. Based on (4.29)-(4.34) the integral $I_{1}$ in (4.28) becomes

$$
I_{1}=\frac{\lambda_{2} \lambda_{3}}{2 \pi} \sigma_{Y}^{2} \int_{-\infty}^{+\infty} \int_{-\infty}^{+\infty} e^{-\sqrt{\gamma^{2}+\chi^{*^{2}}}} e^{-i \boldsymbol{k}^{*} \cdot \chi^{*}} d \chi_{2}^{*} d \chi_{3}^{*} .
$$

Transforming (4.35) into polar coordinates so that the $\chi_{2}^{*}$-axis coincides with the direction of $\boldsymbol{k}^{*}$, we obtain

$$
\begin{aligned}
I_{1} & =\frac{\lambda_{2} \lambda_{3}}{2 \pi} \sigma_{Y}^{2} \int_{\chi^{*}=0}^{+\infty} \int_{\theta=0}^{2 \pi} e^{-\sqrt{\gamma^{2}+\chi^{*}}} e^{-i k^{*} \chi^{*} \cos \theta} \chi^{*} d \chi^{*} d \theta \\
& =\frac{\lambda_{2} \lambda_{3}}{\pi} \sigma_{Y}^{2} \int_{\chi^{*}=0}^{+\infty} \chi^{*} e^{-\sqrt{\gamma^{2}+\chi^{* 2}}}\left\{\int_{\theta=0}^{\pi} e^{-i k^{*} \chi^{*} \cos \theta} d \theta\right\} d \chi^{*} .
\end{aligned}
$$

The integral over $\theta$ is given by [Gradshteyn and Ryzhik, 1980, p. 482[5]]

$$
\int_{\theta=0}^{\pi} e^{-i k^{*} \chi^{*} \cos \theta} d \theta=\pi J_{0}\left(k^{*} \chi^{*}\right)
$$

where $J_{0}\left(k^{*} \chi^{*}\right)$ is Bessel function of order zero, and $J_{0}\left(-k^{*} \chi^{*}\right)=J_{0}\left(k^{*} \chi^{*}\right)$ [Gradshteyn and Ryzhik, 1980, p. 951[8.402]]. Substituting (4.37) into (4.36) yields

$$
I_{1}=\lambda_{2} \lambda_{3} \sigma_{Y}^{2} \int_{\chi^{*}=0}^{+\infty} \chi^{*} e^{-\sqrt{\gamma^{2}+\chi^{* 2}}} J_{0}\left(k^{*} \chi^{*}\right) d \chi^{*} .
$$


From Erdelyi et al. [1954, p. 9[23]], for $\chi_{1} \neq x_{1}($ or $\gamma \neq 0)$, we obtain

$$
\begin{aligned}
& \Lambda\left(-k_{2},-k_{3} ; \chi_{1}, \boldsymbol{x}\right)=e^{-i\left(k_{2} x_{2}+k_{3} x_{3}\right)} \\
& \quad \lambda_{2} \lambda_{3} \sigma_{Y}^{2}\left(1+k^{*^{2}}\right)^{-3 / 2}\left[1+\gamma \sqrt{1+k^{*^{2}}}\right] e^{-\gamma \sqrt{1+k^{* 2}}} .
\end{aligned}
$$

For $\chi_{1}=x_{1}($ or $\gamma=0)$ equation 4.38 becomes

$$
I_{1}=\lambda_{2} \lambda_{3} \sigma_{Y}^{2} \int_{\chi^{*}=0}^{+\infty} \chi^{*} e^{-\chi^{*}} J_{0}\left(k^{*} \chi^{*}\right) d \chi^{*} .
$$

From Erdelyi et al. [1954, p. 9[20]] one finds that

$$
I_{1}=\lambda_{2} \lambda_{3} \sigma_{Y}^{2} \frac{1}{\left(1+k^{*^{2}}\right)} P_{1}\left[\frac{1}{\left(1+k^{*^{2}}\right)^{1 / 2}}\right],
$$

where $P_{1}\left(k^{*}\right)$, the Legendre Polynomial of order one, is evaluated in Appendix D. Substituting (D.7) in (4.41) we obtain (for $\chi_{1}=x_{1}$ )

$$
\Lambda\left(-k_{2},-k_{3} ; \chi_{1}, \boldsymbol{x}\right)=e^{-i\left(k_{2} x_{2}+k_{3} x_{3}\right)} \lambda_{2} \lambda_{3} \sigma_{Y}^{2}\left(1+k^{*^{2}}\right)^{-3 / 2},
$$

and hence (4.39) represents the two-dimensional FT of the exponential covariance function for all $\chi_{1}$.

An independent check of (4.39) is provided in Appendix L by performing an additional FT with respect to $\chi_{1}$, and obtaining

$$
F T^{(3)}(-\boldsymbol{k})=\sqrt{\frac{8}{\pi}} \frac{\lambda_{1} \lambda_{2} \lambda_{3} \sigma_{Y}^{2} e^{-i \boldsymbol{k} \cdot \boldsymbol{x}}}{\left[1+\left(\lambda_{1} k_{1}\right)^{2}+\left(\lambda_{2} k_{2}\right)^{2}+\left(\lambda_{3} k_{3}\right)^{2}\right]^{2}} .
$$

This is the three-dimensional FT of the exponential covariance function (for $\boldsymbol{x}=0$ ) [Gelhar and Axness, 1983a; Gelhar, 1986; Zhang, 1990].

\subsection{Two-Dimensional FT of the Derivatives of $G_{F}$}

The governing equation of the Green's function $G_{F},(4.10)$, can be written as

$$
\nabla_{\chi}^{2} G_{F}(\boldsymbol{x}, \boldsymbol{\chi})=-\delta\left(\chi_{1}-x_{1}\right) \delta\left(\chi_{2}-x_{2}\right) \delta\left(\chi_{3}-x_{3}\right) .
$$


We designate

$$
F T^{(2)}\left[G_{F}\right]=\hat{g}\left(k_{2}, k_{3} ; \chi_{1}, \boldsymbol{x}, a\right),
$$

the two-dimensional FT of $G_{F}$ with respect to $\chi_{2}$ and $\chi_{3}$. From (4.44) we obtain [Haberman, 1987, p. 382]

$$
\frac{\partial^{2}}{\partial \chi_{1}^{2}} \hat{g}-k_{2}^{2} \hat{g}-k_{3}^{2} \hat{g}=-\frac{1}{2 \pi} \delta\left(\chi_{1}-x_{1}\right) e^{i\left(k_{2} x_{2}+k_{3} x_{3}\right)} .
$$

We then rewrite (4.46) as

$$
-\hat{g}^{\prime \prime}+\left(k_{2}^{2}+k_{3}^{2}\right) \hat{g}=c \delta\left(\chi_{1}-x_{1}\right),
$$

where $c$ is a constant,

$$
c=\frac{1}{2 \pi} e^{i\left(k_{2} x_{2}+k_{3} x_{3}\right)} .
$$

Taking the two-dimensional FT of the boundary conditions (4.11) and (4.12) we obtain

$$
\begin{aligned}
& \hat{g}\left(k_{2}, k_{3} ; \chi_{1}=-a, \boldsymbol{x}\right)=0 \\
& \hat{g}\left(k_{2}, k_{3} ; \chi_{1}=+a, \boldsymbol{x}\right)=0 .
\end{aligned}
$$

The solution of

$$
-\frac{d^{2} u}{d \chi^{2}}+\beta^{2} u=\delta(\chi-x),
$$

subject to the boundary conditions

$$
u(-a, x)=0 \quad, \quad u(+a, x)=0,
$$

is given in Appendix $\mathrm{M}$ as

$$
u(\chi, x)=\frac{1}{2 \beta} \sum_{j=-\infty}^{+\infty}(-1)^{j} e^{-\beta\left|\chi-2 j a-(-1)^{j} x\right|},
$$


where $\beta^{2}=k^{\prime 2}=k_{2}^{2}+k_{3}^{2}$ and $\boldsymbol{k}^{\prime}=\left(k_{2}, k_{3}\right)^{T}$ was defined in (4.23).

Based on (4.53) $\hat{g}$ is given by

$$
\hat{g}=\frac{c}{2 k^{\prime}} \sum_{j=-\infty}^{+\infty}(-1)^{j} e^{-k^{\prime}\left|\chi_{1}-2 j a-(-1)^{j} x_{1}\right|} .
$$

Taking the derivative of (4.54) with respect to $\chi_{1}$ gives

$$
\frac{\partial \hat{g}}{\partial \chi_{1}}=-\frac{c}{2} \sum_{j=-\infty}^{+\infty}(-1)^{j}\left[\operatorname{sgn}\left(\chi_{1}-2 j a-(-1)^{j} x_{1}\right)\right] e^{-k^{\prime}\left|\chi_{1}-2 j a-(-1)^{j} x_{1}\right|}
$$

and then

$$
\begin{aligned}
\hat{g}_{x_{1} \chi_{1}} & =\frac{\partial^{2} \hat{g}}{\partial x_{1} \partial \chi_{1}}=-\frac{c k^{\prime}}{2} \sum_{j=-\infty}^{+\infty} e^{-k^{\prime}\left|\chi_{1}-2 j a-(-1)^{j} x_{1}\right|} \\
& +c \sum_{j=-\infty}^{+\infty} \delta\left(\chi_{1}-2 j a-(-1)^{j} x_{1}\right) e^{-k^{\prime}\left|\chi_{1}-2 j a-(-1)^{j} x_{1}\right|}
\end{aligned}
$$

Here we have used the relationships

$$
\frac{\partial}{\partial x_{1}} \operatorname{sgn}\left(\chi_{1}-2 j a-(-1)^{j} x_{1}\right)=-2(-1)^{j} \delta\left(\chi_{1}-2 j a-(-1)^{j} x_{1}\right)
$$

and

$$
\operatorname{sgn}^{2}\left(\chi_{1}-2 j a-(-1)^{j} x_{1}\right)=1 .
$$

The second series in (4.56) is expanded as

$$
\begin{aligned}
& \sum_{j=-\infty}^{+\infty} \delta\left(\chi_{1}-2 j a-(-1)^{j} x_{1}\right) e^{-k^{\prime}\left|\chi_{1}-2 j a-(-1)^{j} x_{1}\right|}= \\
& =+\delta\left(\chi_{1}-x_{1}\right) e^{-k^{\prime}\left|\chi_{1}-x_{1}\right|} \\
& \quad+\delta\left(\chi_{1}-2 a+x_{1}\right) e^{-k^{\prime}\left|\chi_{1}-2 a+x_{1}\right|} \\
& \quad+\delta\left(\chi_{1}+2 a+x_{1}\right) e^{-k^{\prime}\left|\chi_{1}+2 a+x_{1}\right|} \\
& \quad+\sum_{j=-\infty}^{-2}(\cdots)+\sum_{j=+2}^{+\infty}(\cdots)
\end{aligned}
$$


However, for $j= \pm 2$, the delta function applies at $\chi_{1} \mp 4 a-x_{1} \neq 0$ because $-a \leq$ $\chi_{1}, x_{1} \leq+a$, hence the delta function is zero. Similarly, the delta functions are zero for all $j= \pm n, n \geq 2$, and thus the two series at the end of (4.59) drop out. For $-a<x_{1}<+a$, the second and third terms on the right hand side of (4.59) are equal to zero. This results from the fact that

$$
\begin{array}{lll}
\delta\left(\chi_{1}-2 a+x_{1}\right) \neq 0 & \text { only for } & \chi_{1}+x_{1}=+2 a \\
\delta\left(\chi_{1}+2 a+x_{1}\right) \neq 0 & \text { only for } & \chi_{1}+x_{1}=-2 a
\end{array}
$$

and hence the delta functions are zero for $-a<x_{1}<+a$.

We now have that

$$
\delta\left(\chi_{1}-x_{1}\right) \neq 0 \quad \text { only for } \quad \chi_{1}-x_{1}=0
$$

and thus, for $\chi_{1} \neq x_{1},(4.56)$ becomes

$$
\hat{g}_{x_{1} \chi_{1}}=-\frac{c k^{\prime}}{2} \sum_{j=-\infty}^{+\infty} e^{-k^{\prime}\left|\chi_{1}-2 j a-(-1)^{j} x_{1}\right|}
$$

For $\chi_{1}=x_{1},(4.56)$ is given by

$$
\hat{g}_{x_{1} \chi_{1}}=c \delta\left(\chi_{1}-x_{1}\right)-\frac{c k^{\prime}}{2} \sum_{j=-\infty}^{+\infty} e^{-k^{\prime}\left|\chi_{1}-2 j a-(-1)^{j} x_{1}\right|} .
$$

Combining (4.63) and (4.64) and substituting $c$ from (4.48) we obtain, for all $\chi_{1}$,

$$
\begin{aligned}
\hat{g}_{x_{1} \chi_{1}}= & \frac{1}{2 \pi} e^{i\left(k_{2} x_{2}+k_{3} x_{3}\right)}\left\{\delta\left(\chi_{1}-x_{1}\right)\right. \\
& \left.-\frac{k^{\prime}}{2} \sum_{j=-\infty}^{+\infty} e^{-k^{\prime}\left|\chi_{1}-2 j a-(-1)^{j} x_{1}\right|}\right\} .
\end{aligned}
$$

The result for $\chi_{1} \neq x_{1}$ in (4.63) was also derived by an alternate method presented in Appendix P. The approach in Appendix $\mathrm{P}$ has a disadvantage in that it requires the evaluation of integrals which, for $\chi_{1}=x_{1}$, do not appear in any available tables. 
The infinite series in (4.65) is evaluated in closed form in Appendix O. This allows rewriting (4.65) as

$$
\begin{aligned}
\hat{g}_{x_{1} \chi_{1}}= & \frac{1}{2 \pi} e^{i\left(k_{2} x_{2}+k_{3} x_{3}\right)}\left\{\delta\left(\chi_{1}-x_{1}\right)-\frac{k^{\prime}}{2} e^{-k^{\prime}\left|\chi_{1}-x_{1}\right|}\right. \\
& \left.-\frac{k^{\prime}}{2} \frac{\cosh \left[k^{\prime}\left(\chi_{1}+x_{1}\right)\right]}{\sinh \left[2 k^{\prime} a\right]}-\frac{k^{\prime}}{2} e^{-2 k^{\prime} a} \frac{\cosh \left[k^{\prime}\left(\chi_{1}-x_{1}\right)\right]}{\sinh \left[2 k^{\prime} a\right]}\right\} .
\end{aligned}
$$

However, this does not make the evaluation of integrals any easier, and we shall therefore use (4.65) in the subsequent analysis.

When the plates are far from each other, only the index $j=0$ in the infinite series of (4.65) applies, and (4.65) takes the simpler form

$$
\begin{aligned}
\hat{g}_{x_{1} \chi_{1}}= & \frac{1}{2 \pi} e^{i\left(k_{2} x_{2}+k_{3} x_{3}\right)}\left\{\delta\left(\chi_{1}-x_{1}\right)\right. \\
& \left.-\frac{\left(k_{2}^{2}+k_{3}^{2}\right)^{1 / 2}}{2} e^{-\left(k_{2}^{2}+k_{3}^{2}\right)^{1 / 2}\left|\chi_{1}-x_{1}\right|}\right\},
\end{aligned}
$$

where $k^{\prime}=\left(k_{2}^{2}+k_{3}^{2}\right)^{1 / 2}$. A check of (4.67) is provided by taking its one-dimensional FT with respect to $\chi_{\mathbf{1}}($ Appendix $\mathrm{N})$ to obtain the three-dimensional FT of the mixed derivatives of the infinite Green's function.

\subsection{Evaluation of Surface and Domain Integrals}

Upon expressing $\boldsymbol{k}^{\prime}$ in polar coordinates,

$$
\boldsymbol{k}^{\prime}=k^{\prime} \boldsymbol{\sigma}=k^{\prime}(\cos \theta, \sin \theta)^{T}
$$

the norm of $\boldsymbol{k}^{*},(4.34)$, becomes

$$
\begin{aligned}
k^{*^{2}} & =\left\|\boldsymbol{k}^{*^{2}}\right\|=k^{\prime 2}\left\|\boldsymbol{L}^{\prime} \boldsymbol{\sigma}\right\|^{2} \\
& =k^{\prime 2}\left(\boldsymbol{L}^{\prime} \boldsymbol{\sigma}\right)^{T}\left(\boldsymbol{L}^{\prime} \boldsymbol{\sigma}\right) \\
& =k^{\prime 2}\left(\boldsymbol{\sigma}^{T} \boldsymbol{L}^{\prime 2} \boldsymbol{\sigma}\right) .
\end{aligned}
$$


Setting

$$
\boldsymbol{\lambda}=\left(\begin{array}{cc}
\lambda_{1} / \lambda_{2} & 0 \\
0 & \lambda_{1} / \lambda_{3}
\end{array}\right)
$$

the above norm becomes

$$
k^{*^{2}}=k^{\prime 2} \lambda_{1}^{2} R^{2} .
$$

The scalar $R^{2}$ is given by

$$
R^{2}=\boldsymbol{\sigma}^{T} \boldsymbol{\lambda}^{-2} \boldsymbol{\sigma}
$$

or, explicitly, by

$$
R^{2}=\varepsilon_{2}^{2} \cos ^{2} \theta+\varepsilon_{3}^{2} \sin ^{2} \theta .
$$

Here we have set

$$
\varepsilon_{2}=\frac{\lambda_{2}}{\lambda_{1}} \quad ; \quad \varepsilon_{3}=\frac{\lambda_{3}}{\lambda_{1}} .
$$

It is clear that $R^{2}$ is strictly positive for integral scales different from zero. Matrix $\boldsymbol{\lambda}^{-2}$ is given by

$$
\boldsymbol{\lambda}^{-2}=\frac{1}{\lambda_{1}^{2}} \boldsymbol{L}^{\prime 2}
$$

We define the dimensionless parameters

$$
\begin{gathered}
\rho=\frac{a}{\lambda_{1}}>0, \\
-1 \leq \rho_{\chi}=\frac{\chi_{1}}{a} \leq+1, \\
-1<\rho_{x}=\frac{x_{1}}{a}<+1,
\end{gathered}
$$

where the singularity point is assumed not to occur on the Dirichlet boundaries. The dimensionless parameters $\rho, \varepsilon_{2}$, and $\varepsilon_{3}$ are recognized by Smith and Freeze [1979a, b] to be fundamental parameters describing flow within a bounded domain: "the output distributions of the prediction variables must be interpreted in light of these parameters". 
We rewrite (4.39) as

$$
\Lambda\left(-k_{2},-k_{3} ; \chi_{1}, \boldsymbol{x}\right)=e^{-i\left(k_{2} x_{2}+k_{3} x_{3}\right)} \Lambda^{\prime}\left(k_{2}, k_{3} ; \eta_{1}\right)
$$

where

$$
\begin{aligned}
\Lambda^{\prime}\left(k_{2}, k_{3} ; \eta_{1}\right)= & \lambda_{2} \lambda_{3} \sigma_{Y}^{2}\left(1+k^{*^{2}}\right)^{-3 / 2} \\
& {\left[1+\gamma \sqrt{\left.1+k^{*^{2}}\right]} e^{-\gamma \sqrt{1+k^{*^{2}}}} .\right.}
\end{aligned}
$$

We also rewrite (4.65) as

$$
F\left(k_{2}, k_{3} ; \chi_{1}, \boldsymbol{x}\right)=e^{i\left(k_{2} x_{2}+k_{3} x_{3}\right)} F^{\prime}\left(k_{2}, k_{3} ; \chi_{1}, x_{1}\right)
$$

where

$$
F^{\prime}\left(k_{2}, k_{3} ; \chi_{1}, x_{1}\right)=\frac{1}{2 \pi}\left\{\delta\left(\chi_{1}-x_{1}\right)-\frac{k^{\prime}}{2} \sum_{j=-\infty}^{+\infty} e^{-k^{\prime}\left|\chi_{1}-2 j a-(-1)^{j} x_{1}\right|}\right\}
$$

Based on (4.79)-(4.82) the integral $\mathcal{H}$ in (4.27) becomes

$$
\begin{aligned}
\mathcal{H}\left(\chi_{1}, x_{1}, a\right) & =\int_{\boldsymbol{k}^{\prime}} \Lambda F d \boldsymbol{k}^{\prime}=\int_{\boldsymbol{k}^{\prime}} \Lambda^{\prime} F^{\prime} d \boldsymbol{k}^{\prime} \\
& =\frac{1}{2 \pi} \delta\left(\eta_{1}\right) \int_{\boldsymbol{k}^{\prime}} \Lambda^{\prime}\left(\boldsymbol{k}^{\prime} ; \eta_{1}\right) d \boldsymbol{k}^{\prime} \\
& -\frac{1}{4 \pi} \sum_{j=-\infty}^{+\infty} \int_{\boldsymbol{k}^{\prime}} k^{\prime} e^{-k^{\prime}\left|\chi_{1}-2 j a-(-1)^{j} x_{1}\right|} \Lambda^{\prime}\left(\boldsymbol{k}^{\prime} ; \eta_{1}\right) d \boldsymbol{k}^{\prime}
\end{aligned}
$$

\subsubsection{General Expression for the Surface Integral}

The first term of (4.83) is given by

$$
\begin{aligned}
\Delta & =\delta\left(\eta_{1}\right)\left\{\frac{1}{2 \pi} \int_{\boldsymbol{k}^{\prime}} \Lambda^{\prime}\left(\boldsymbol{k}^{\prime} ; \eta_{1}\right) d \boldsymbol{k}^{\prime}\right\} \\
& =\delta\left(\chi_{1}-x_{1}\right) C_{Y}\left(\chi_{1}-x_{1}, 0,0\right)
\end{aligned}
$$


Here $\eta_{1}=\chi_{1}-x_{1}$ and the term within braces, from the definition of the inverse FT in (B.1), equals $C_{Y}\left(\chi_{1}-x_{1}, 0,0\right)$. Substituting (3.32) into (4.84) we obtain

$$
\begin{aligned}
\Delta & =\delta\left(\chi_{1}-x_{1}\right) C_{Y}\left(\chi_{1}-x_{1}, 0,0\right) \\
& =\delta\left(\eta_{1}\right) \sigma_{Y}^{2} \exp \left(-\frac{\chi_{1}-x_{1}}{\lambda_{1}}\right)
\end{aligned}
$$

Substituting (4.83) and (4.85) into the surface integral $S,(4.17)$, yields

$$
\begin{gathered}
S\left(x_{1}, a\right)=-2 a \delta\left(\eta_{1}\right) \sigma_{Y}^{2} \exp \left(-\frac{\chi_{1}-x_{1}}{\lambda_{1}}\right)+\frac{a}{2 \pi} \sum_{j=-\infty}^{+\infty} \\
\int_{\boldsymbol{k}^{\prime}} k^{\prime} e^{-k^{\prime}\left|\chi_{1}-2 j a-(-1)^{j_{1}} x_{1}\right|} \Lambda^{\prime}\left(\boldsymbol{k}^{\prime} ; \eta_{1}\right) d \boldsymbol{k}^{\prime},
\end{gathered}
$$

where the right hand side of (4.86) must be evaluated at $\chi_{1}=a$.

We need to account for the existence of the Dirac delta function $\delta\left(\eta_{1}\right)$ in our expression for the surface integral. To this end, we define a delta-sequence [Greenberg, 1971], $\delta_{a}\left(\eta_{1}\right)$,

$$
\delta_{a}\left(\eta_{1}\right)= \begin{cases}1 / 2 a & \text { for }\left|\eta_{1}\right|<a \\ 0 & \text { otherwise }\end{cases}
$$

This delta-sequence is depicted in Figure 4.3.

We borrow some terminology from Zauderer [1983, p. 365] to define the concept of a delta-sequence and its relationship to the Dirac delta. We begin by considering the collection of test functions $\phi(x)$, assumed to be infinitely differentiable and to vanish identically outside a bounded region. The Dirac delta, $\delta(x)$, belongs to a class of functions called generalized functions which are determined by specifying their effect on the test function $\phi(x)$ through the linear functional $(\delta, \phi)$. Strictly speaking, a generalized function is not a function of $x$ (i.e., it does not assume values for each $x$ ) but rather of each test function $\phi(x)$. Given a delta-sequence, $\delta_{a}(x)$, for 


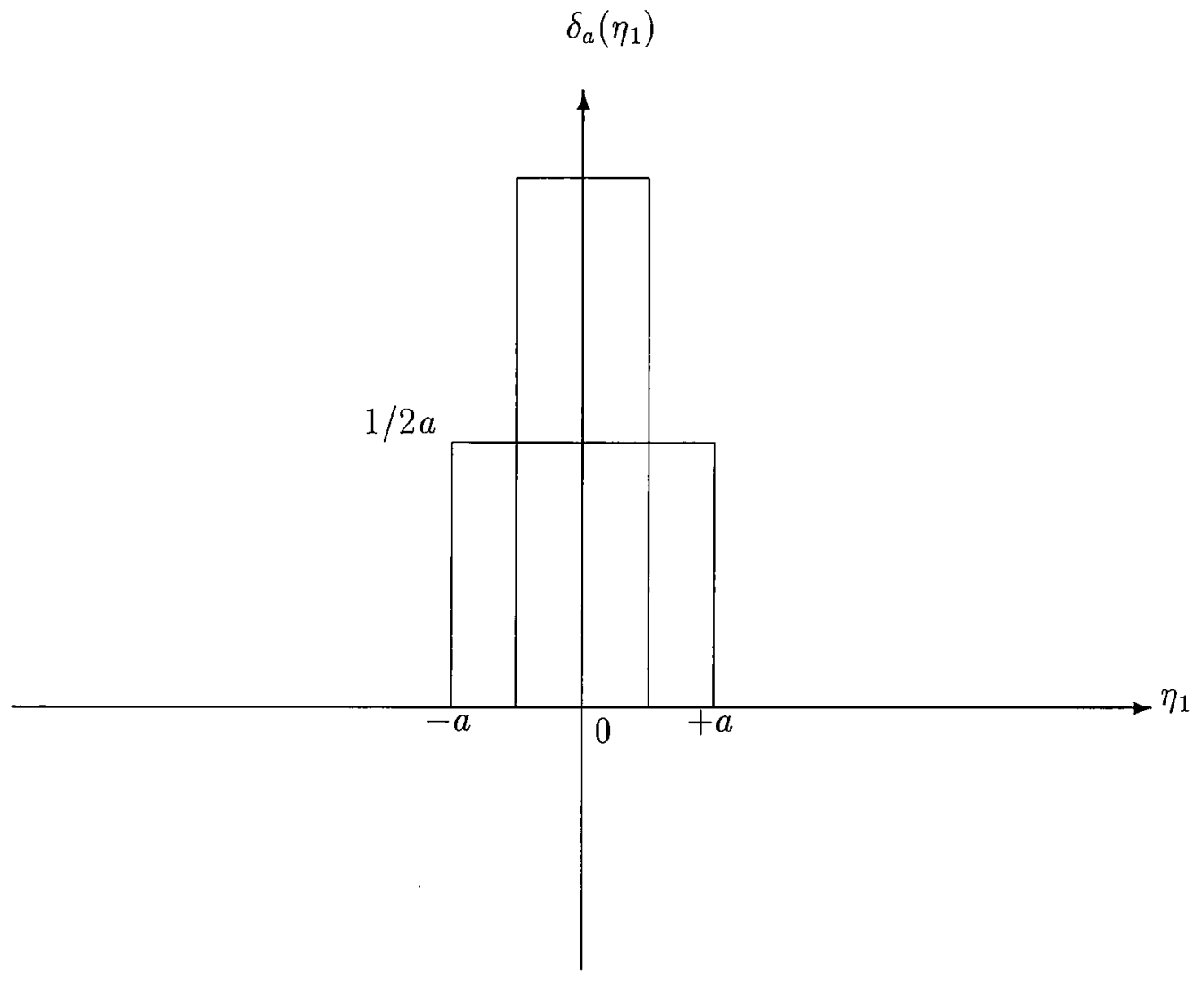

Figure 4.3: Dirac delta sequence $\delta_{a}\left(\eta_{1}\right)$. 
which

$$
\lim _{a \rightarrow 0}\left(\delta_{a}, \phi\right)=(\delta, \phi),
$$

we say that $\delta_{a}(x)$ converges to the generalized function $\delta(x)$ as $a \rightarrow 0$. Let us consider the operation of the delta-sequence (4.87) on the test function $\phi(x)$,

$$
\begin{aligned}
\left(\delta_{a}, \phi\right) & =\int_{-\infty}^{+\infty} \delta_{a}(x) \phi(x) d x \\
& =\frac{1}{2 a} \int_{-a}^{+a} \phi(x) d x=\frac{\phi(\hat{x})}{2 a} \int_{-a}^{+a} d x=\phi(\hat{x}),
\end{aligned}
$$

where $\hat{x} \in[-a, a]$ and the third equality arises from the mean value theorem [Gaughan, 1987].

Since $\phi(x)$ is continuous at $x=0$ we obtain

$$
\lim _{a \rightarrow 0}\left(\delta_{a}, \phi\right)=\lim _{a \rightarrow 0} \phi(\hat{x})=\phi(0)=(\delta, \phi) .
$$

Recalling the definition of the convergence of sequences of generalized functions in (4.88), we conclude that (4.87) converges to the delta function as $a \rightarrow 0$. We have shown earlier (pages 71 and 76 ) that the effective hydraulic conductivity $K_{e}$ does not depend on the coordinate $x_{1}$ but only on $a$. For any fixed point $x_{1}=\hat{x} \in[-a,+a]$ and for $\chi_{1}=+a$ the first term of the right hand side of (4.86) equals $c \delta\left(\eta_{1}\right)$. Here $c=-2 a \sigma_{Y}^{2} \exp \left\{(\hat{x}-a) / \lambda_{1}\right\}$ is a constant (for fixed $a$ and $\hat{x}$ ). For finite distances $2 a$ between the boundaries, by virtue of the relationship

$$
\int_{-\infty}^{+\infty} \delta\left(\eta_{1}\right) c d \eta_{1}=\int_{-a}^{+a} \frac{1}{2 a} c d \eta_{1}=c
$$

expression (4.87) is seen to operate on the constant term $c$ of the right hand side of (4.86) in the same manner as the delta function $\delta\left(\eta_{1}\right)$. When $a \rightarrow \infty$ expression (4.87) behaves the same as the delta function $\delta\left(\eta_{1}\right)$ and the first term of the right 
hand side of (4.86) equals zero. Hence, for any $a$, we can substitute the Dirac delta in (4.86) by the delta sequence of (4.87).

On a less rigorous note, we observe that (Figure 4.3) the delta-sequence $\delta_{a}\left(\eta_{1}\right)$ exhibits correct limit behavior. When $2 a$ tends to zero, $\delta_{a}\left(\eta_{1}\right)$ tends to infinity, and such behavior corresponds to the case where the two planes come arbitrarily close together, hence the influence of the boundaries becomes extremely large. When the two planes are at a large distance from each other ( $2 a$ tends to infinity), the influence of the delta-sequence is not felt. Finally, $\delta_{a}\left(\eta_{1}\right)$ satisfies

$$
\int_{-a}^{+a} \delta_{a}\left(\eta_{1}\right) d \eta_{1}=\int_{-a}^{+a} \frac{1}{2 a} d \eta_{1}=1
$$

and hence, behaves exactly like a Dirac delta function.

Upon substitution of (4.87) into (4.86), the surface integral $S$ becomes

$$
\begin{aligned}
S\left(x_{1}, a\right)= & -\sigma_{Y}^{2} e^{-\rho\left(1-\rho_{x}\right)}+\frac{a}{2 \pi} \sum_{j=-\infty}^{+\infty} \\
& \int_{\boldsymbol{k}^{\prime}} k^{\prime} e^{-k^{\prime}\left|a-2 j a-(-1)^{j} x_{1}\right|} \Lambda^{\prime}\left(\boldsymbol{k}^{\prime} ; \eta_{1}\right) d \boldsymbol{k}^{\prime} .
\end{aligned}
$$

Here equations 4.76 , and 4.78 were used, and $\chi_{1}$ was replaced by its value on $\Gamma_{D_{1}}^{+}$, i.e., $\chi_{1}=+a$. Transforming (4.93) into polar coordinates, and substituting (4.80), we obtain

$$
\begin{aligned}
& S\left(x_{1}, a\right)=-\sigma_{Y}^{2} e^{-\rho\left(1-\rho_{x}\right)}+\frac{a \lambda_{2} \lambda_{3}}{2 \pi} \sigma_{Y}^{2} \sum_{j=-\infty}^{+\infty} \int_{k^{\prime}=0}^{\infty} \int_{\theta=0}^{2 \pi} \\
& k^{\prime 2}\left(1+k^{*^{2}}\right)^{-3 / 2}\left[1+\gamma \sqrt{1+k^{*}}\right] e^{-\gamma \sqrt{1+k^{*}}} \\
& e^{-k^{\prime}\left|a-2 j a-(-1)^{j} x_{1}\right|} d k^{\prime} d \theta .
\end{aligned}
$$

Setting $\chi_{1}=+a$, and with the help of (4.76), and (4.78) we rewrite $\gamma$ as

$$
\gamma=\frac{\left|a-x_{1}\right|}{\lambda_{1}}=\frac{a}{\lambda_{1}}\left|1-\frac{x_{1}}{a}\right|=\rho\left|1-\rho_{x}\right| .
$$


With the use of (4.71) and (4.95), equation 4.94 becomes

$$
\begin{gathered}
S\left(x_{1}, a\right)=-\sigma_{Y}^{2} e^{-\rho\left(1-\rho_{x}\right)}+\frac{a \lambda_{2} \lambda_{3}}{2 \pi} \sigma_{Y}^{2} \sum_{j=-\infty}^{+\infty} \int_{\theta=0}^{2 \pi} \int_{k^{\prime}=0}^{\infty} \\
k^{\prime 2}\left[1+\left(k^{\prime} \lambda_{1} R\right)^{2}\right]^{-3 / 2}\left[1+\rho\left|1-\rho_{x}\right| \sqrt{1+\left(k^{\prime} \lambda_{1} R\right)^{2}}\right] \\
e^{-\rho\left|1-\rho_{x}\right| \sqrt{1+\left(k^{\prime} \lambda_{1} R\right)^{2}}} e^{-a k^{\prime}\left|1-2 j-(-1)^{j} \rho_{x}\right|} d k^{\prime} d \theta .
\end{gathered}
$$

Performing the transformation $x=\lambda_{1} k^{\prime}$, selecting, without loss of generality, $x_{1}=0$ (or $\rho_{x}=0$ ), and using the relationship

$$
\frac{a \lambda_{2} \lambda_{3}}{\lambda_{1}^{3}}=\rho \varepsilon_{2} \varepsilon_{3},
$$

a dimensionless expression for the surface integral $S$, for statistically anisotropic media, is finally given by

$$
\begin{gathered}
S(a)=-\sigma_{Y}^{2} e^{-\rho}+\frac{\sigma_{Y}^{2}}{2 \pi} \rho \varepsilon_{2} \varepsilon_{3} \sum_{j=-\infty}^{+\infty} \int_{\theta=0}^{2 \pi} \int_{x=0}^{\infty} \\
x^{2}\left[1+(x R)^{2}\right]^{-3 / 2}\left[1+\rho \sqrt{1+(x R)^{2}}\right] \\
e^{-\rho \sqrt{1+(x R)^{2}}} e^{-\rho|1-2 j| x} d x d \theta .
\end{gathered}
$$

For statistically isotropic media we have $\varepsilon_{2}=\varepsilon_{3}=1$ and $R=1$. Then the surface integral is given by

$$
\begin{gathered}
S(a)=-\sigma_{Y}^{2} e^{-\rho}+\rho \sigma_{Y}^{2} \sum_{j=-\infty}^{+\infty} \int_{x=0}^{\infty} \\
x^{2}\left[1+x^{2}\right]^{-3 / 2}\left[1+\rho \sqrt{1+x^{2}}\right] \\
e^{-\rho \sqrt{1+x^{2}}} e^{-\rho|1-2 j| x} d x
\end{gathered}
$$

\subsubsection{General Expression for the Domain Integral}

We recall that the domain integral $D,(4.18)$, is given by

$$
D=\int_{\chi_{1}=-a}^{\chi_{1}=+a} \mathcal{H} d \chi_{1} .
$$


Upon substituting $\mathcal{H}$ from (4.83), this becomes

$$
\begin{aligned}
D\left(x_{1}, a\right)= & \frac{1}{2 \pi} \int_{\boldsymbol{k}^{\prime}} \int_{\chi_{1}=-a}^{\chi_{1}=+a} \delta\left(\eta_{1}\right) \Lambda^{\prime}\left(\boldsymbol{k}^{\prime} ; \eta_{1}\right) d \boldsymbol{k}^{\prime} d \chi_{1}-\frac{1}{4 \pi} \sum_{j=-\infty}^{+\infty} \\
& \int_{\boldsymbol{k}^{\prime}} \int_{\chi_{1}=-a}^{\chi_{1}=+a} k^{\prime} e^{-k^{\prime}\left|\chi_{1}-2 j a-(-1)^{j} x_{1}\right|} \Lambda^{\prime}\left(\boldsymbol{k}^{\prime} ; \eta_{1}\right) d \boldsymbol{k}^{\prime} d \chi_{1} .
\end{aligned}
$$

From the property of the delta function we have that

$$
\int_{\chi_{1}=-a}^{\chi_{1}=+a} \delta\left(\eta_{1}\right) \Lambda^{\prime}\left(\boldsymbol{k}^{\prime} ; \eta_{1}\right) d \chi_{1}=\Lambda^{\prime}\left(\boldsymbol{k}^{\prime} ; 0\right)
$$

Then the first term of the right hand side of (4.101) becomes

$$
\frac{1}{2 \pi} \int_{\boldsymbol{k}^{\prime}} \Lambda^{\prime}\left(\boldsymbol{k}^{\prime} ; 0\right) d \boldsymbol{k}^{\prime}=C_{Y}(0,0,0)=\sigma_{Y}^{2}
$$

Here we recall that $\Lambda^{\prime}$ in $(4.80)$ is the two-dimensional FT of the exponential covariance function, hence (B.1) and (3.32) lead to (4.103). Considering (4.103) equation 4.101 simplifies to

$$
\begin{aligned}
D\left(x_{1}, a\right)= & \sigma_{Y}^{2}-\frac{1}{4 \pi} \sum_{j=-\infty}^{+\infty} \int_{\boldsymbol{k}^{\prime}} \int_{\chi_{1}=-a}^{\chi_{1}=+a} k^{\prime} \\
& e^{-k^{\prime}\left|\chi_{1}-2 j a-(-1)^{j} x_{1}\right|} \Lambda^{\prime}\left(\boldsymbol{k}^{\prime} ; \eta_{1}\right) d \boldsymbol{k}^{\prime} d \chi_{1} .
\end{aligned}
$$

Transforming into polar coordinates, and substituting (4.80), we obtain

$$
\begin{gathered}
D\left(x_{1}, a\right)=\sigma_{Y}^{2}-\frac{\lambda_{2} \lambda_{3}}{4 \pi} \sigma_{Y}^{2} \sum_{j=-\infty}^{+\infty} \int_{\chi_{1}=-a}^{\chi_{1}=+a} \int_{k^{\prime}=0}^{\infty} \int_{\theta=0}^{2 \pi} k^{\prime 2} \\
\left(1+k^{*^{2}}\right)^{-3 / 2}\left[1+\gamma \sqrt{1+k^{*^{2}}}\right] e^{-\gamma \sqrt{1+k^{*}}} \\
e^{-k^{\prime}\left|\chi_{1}-2 j a-(-1)^{j} x_{1}\right|} d k^{\prime} d \theta d \chi_{1} .
\end{gathered}
$$

Using the definitions in (4.76)-(4.78), expressions (4.71) and (4.97), the transformation $x=\lambda_{1} k^{\prime}$, and selecting $x_{1}=0$ (or $\rho_{x}=0$ ), the domain integral $D$ for statistically 
anisotropic media is finally given by

$$
\begin{gathered}
D(a)=\sigma_{Y}^{2}-\frac{\sigma_{Y}^{2}}{4 \pi} \rho \varepsilon_{2} \varepsilon_{3} \sum_{j=-\infty}^{+\infty} \int_{\rho_{\chi}=-1}^{\rho_{\chi}=+1} \int_{\theta=0}^{2 \pi} \int_{x=0}^{\infty} x^{2} \\
{\left[1+(x R)^{2}\right]^{-3 / 2}\left[1+\rho\left|\rho_{\chi}\right| \sqrt{1+(x R)^{2}}\right]} \\
e^{-\rho\left|\rho_{\chi}\right| \sqrt{1+(x R)^{2}}} e^{-\rho\left|\rho_{\chi}-2 j\right| x} d x d \theta d \rho_{\chi}
\end{gathered}
$$

For statistically isotropic media, $D$ takes the simpler form

$$
\begin{gathered}
D(a)=\sigma_{Y}^{2}-\rho \frac{\sigma_{Y}^{2}}{2} \sum_{j=-\infty}^{+\infty} \int_{\rho_{\chi}=-1}^{\rho_{\chi}=+1} \int_{x=0}^{\infty} x^{2} \\
{\left[1+x^{2}\right]^{-3 / 2}\left[1+\rho\left|\rho_{\chi}\right| \sqrt{1+x^{2}}\right]} \\
e^{-\rho\left|\rho_{\chi}\right| \sqrt{1+x^{2}}} e^{-\rho\left|\rho_{\chi}-2 j\right| x} d x d \rho_{\chi} .
\end{gathered}
$$

\subsection{General Expression for Effective Conductivity}

We made clear earlier (page 76) that the effective hydraulic conductivity $K_{e}$ in our case assumes a constant value. Hence we have set, without loss of generality, in (4.98), (4.99), (4.106) and (4.107) $\rho_{x}=0$. Summarizing now, our expression for the surface integral is given by

$$
S(a)=\sigma_{Y}^{2} \mathcal{S}(a)
$$

where for statistically anisotropic media

$$
\begin{gathered}
\mathcal{S}(a)=-e^{-\rho}+\frac{\rho \varepsilon_{2} \varepsilon_{3}}{2 \pi} \sum_{j=-\infty}^{+\infty} \int_{\theta=0}^{2 \pi} \int_{x=0}^{\infty} \\
x^{2}\left[1+(x R)^{2}\right]^{-3 / 2}\left[1+\rho \sqrt{1+(x R)^{2}}\right] \\
e^{-\rho \sqrt{1+(x R)^{2}}} e^{-\rho|1-2 j| x} d x d \theta
\end{gathered}
$$

and for statistically isotropic media

$$
\mathcal{S}(a)=-e^{-\rho}+\rho \sum_{j=-\infty}^{+\infty} \int_{x=0}^{\infty} x^{2}
$$




$$
\begin{aligned}
& {\left[1+x^{2}\right]^{-3 / 2}\left[1+\rho \sqrt{1+x^{2}}\right]} \\
& e^{-\rho \sqrt{1+x^{2}}} e^{-\rho|1-2 j| x} d x .
\end{aligned}
$$

Likewise, the domain integral is given by

$$
D(a)=\sigma_{Y}^{2} \mathcal{D}(a),
$$

where for statistically anisotropic media

$$
\begin{gathered}
\mathcal{D}(a)=1-\frac{\rho \varepsilon_{2} \varepsilon_{3}}{4 \pi} \sum_{j=-\infty}^{+\infty} \int_{\rho_{\chi}=-1}^{\rho_{\chi}=+1} \int_{\theta=0}^{2 \pi} \int_{x=0}^{\infty} x^{2} \\
{\left[1+(x R)^{2}\right]^{-3 / 2}\left[1+\rho\left|\rho_{\chi}\right| \sqrt{1+(x R)^{2}}\right]} \\
e^{-\rho\left|\rho_{\chi}\right| \sqrt{1+(x R)^{2}}} e^{-\rho\left|\rho_{\chi}-2 j\right| x} d x d \theta d \rho_{\chi}
\end{gathered}
$$

and for statistically isotropic media

$$
\begin{gathered}
\mathcal{D}(a)=1-\frac{\rho}{2} \sum_{j=-\infty}^{+\infty} \int_{\rho_{\chi}=-1}^{\rho_{\chi}=+1} \int_{x=0}^{\infty} x^{2} \\
{\left[1+x^{2}\right]^{-3 / 2}\left[1+\rho\left|\rho_{\chi}\right| \sqrt{1+x^{2}}\right]} \\
e^{-\rho\left|\rho_{\chi}\right| \sqrt{1+x^{2}}} e^{-\rho\left|\rho_{\chi}-2 j\right| x} d x d \rho_{\chi} .
\end{gathered}
$$

The linearized $K_{e}^{L}$ in (4.19) becomes

$$
K_{e}^{L}(a)=K_{G}\left\{1+\sigma_{Y}^{2}\left[\frac{1}{2}-(\mathcal{D}+\mathcal{S})\right]\right\},
$$

and for large $\sigma_{Y}^{2}, K_{e}$ in (4.20) is given by

$$
K_{e}(a)=K_{G} \exp \left\{\sigma_{Y}^{2}\left[\frac{1}{2}-(\mathcal{D}+\mathcal{S})\right]\right\} .
$$




\section{CHAPTER 5}

\section{ASYMPTOTIC ANALYSIS AND NUMERICAL RESULTS}

\subsection{Boundaries Come Arbitrarily Close Together}

In this section we obtain the limits of the effective hydraulic conductivity as $\rho \rightarrow 0$. We proceed by showing that the domain and surface integrals are bounded. Then by invoking the Dominated Convergence Theorem [Ray, 1988, p. 202] we interchange in (4.112) and (4.109) the order of limit and integration.

We consider (4.112),

$$
\mathcal{D}(a)=1-\sum_{j=-\infty}^{+\infty} I_{j}
$$

where

$$
\begin{aligned}
I_{j}= & \frac{\rho \varepsilon_{2} \varepsilon_{3}}{4 \pi} \int_{\rho_{\chi}=-1}^{\rho_{\chi}=+1} \int_{\theta=0}^{2 \pi} \int_{x=0}^{\infty} \frac{x^{2}}{\left[1+(x R)^{2}\right]^{3 / 2}} \\
& {\left[1+\rho\left|\rho_{\chi}\right| \sqrt{1+(x R)^{2}}\right] e^{-\rho\left|\rho_{\chi}\right| \sqrt{1+(x R)^{2}}} e^{-\rho\left|\rho_{\chi}-2 j\right| x} d x d \theta d \rho_{\chi} . }
\end{aligned}
$$

It can easily be seen that inequality $\left|\rho_{\chi}-2 j\right| \geq\left|\rho_{\chi}\right|$ holds for all $j$. From this it follows that $\exp \left(-\rho\left|\rho_{\chi}-2 j\right| x\right) \leq \exp \left(-\rho\left|\rho_{\chi}\right| x\right)(\rho, x \geq 0)$. Hence $I_{0}$ bounds from above all other integrals, or $I_{j} \leq I_{0}$ for all $j$.

Let us consider that $\lambda_{i} \neq 0, i=1,2,3$. We designate $\varepsilon=\min \left(\varepsilon_{2}, \varepsilon_{3}\right)$ and $\varepsilon^{\prime}=\max \left(\varepsilon_{2}, \varepsilon_{3}\right)$. The following inequalities are then satisfied:

$$
\varepsilon^{\prime 2} \geq R^{2}=\varepsilon_{2}^{2} \cos ^{2} \theta+\varepsilon_{3}^{2} \sin ^{2} \theta \geq \varepsilon^{2},
$$




$$
\begin{aligned}
{\left[1+\rho\left|\rho_{\chi}\right| \sqrt{1+\left(x \varepsilon^{\prime}\right)^{2}}\right] } & \geq\left[1+\rho\left|\rho_{\chi}\right| \sqrt{1+(x R)^{2}}\right], \\
{\left[1+(x R)^{2}\right]^{3 / 2} } & \geq\left[1+(x \varepsilon)^{2}\right]^{3 / 2}, \\
\frac{1}{\left[1+(x \varepsilon)^{2}\right]^{3 / 2}} & \geq \frac{1}{\left[1+(x R)^{2}\right]^{3 / 2}}, \\
\rho\left|\rho_{\chi}\right| \sqrt{1+(x R)^{2}} & \geq \rho\left|\rho_{\chi}\right| \sqrt{1+(x \varepsilon)^{2}}, \\
\exp \left(-\rho\left|\rho_{\chi}\right| \sqrt{1+(x \varepsilon)^{2}}\right) & \geq \exp \left(-\rho\left|\rho_{\chi}\right| \sqrt{1+(x R)^{2}}\right) .
\end{aligned}
$$

Substituting (5.4), (5.6), and (5.8) into $(5.2)(j=0)$ we obtain

$$
I_{0} \leq M
$$

where

$$
M=\frac{\varepsilon^{\prime 2}}{2} \int_{x=0}^{\infty} \frac{x^{2}}{\left[1+(x \varepsilon)^{2}\right]^{3 / 2}} K d x
$$

and

$$
\begin{aligned}
K= & \rho \int_{\rho_{\chi}=-1}^{\rho_{\chi}=+1}\left[1+\rho\left|\rho_{\chi}\right| \sqrt{1+\left(x \varepsilon^{\prime}\right)^{2}}\right] \\
& \exp \left\{-\rho\left|\rho_{\chi}\right|\left(x+\sqrt{1+(x \varepsilon)^{2}}\right)\right\} d \rho_{\chi} .
\end{aligned}
$$

Setting $A=\sqrt{1+\left(x \epsilon^{\prime}\right)^{2}}$ and $B=\sqrt{1+(x \varepsilon)^{2}}$, the integral $K$ is found to satisfy [Macsyma, 1992]

$$
\begin{aligned}
K & =\rho \int_{\rho_{\chi}=-1}^{\rho_{\chi}=+1}\left[1+\rho\left|\rho_{\chi}\right| A\right] \exp \left\{-\rho\left|\rho_{\chi}\right|(x+B)\right\} d \rho_{\chi} \\
& \leq 2 \frac{x+A+B}{(x+B)^{2}} .
\end{aligned}
$$

We have that

$$
A+B \leq 2 A
$$




$$
\begin{aligned}
1+(x \varepsilon)^{2} & \geq(x \varepsilon)^{2} \\
x+\sqrt{1+(x \varepsilon)^{2}} & \geq x \varepsilon \\
(x+B)^{2} & \geq(x \varepsilon)^{2} \\
\frac{1}{(x+B)^{2}} & \leq \frac{1}{(x \varepsilon)^{2}} .
\end{aligned}
$$

Upon substituting (5.12)-(5.14) into (5.10) we obtain

$$
\begin{aligned}
M & \leq \frac{\varepsilon^{\prime 2}}{\varepsilon^{2}} \int_{x=0}^{\infty} \frac{x+2 A}{\left[1+(x \varepsilon)^{2}\right]^{3 / 2}} d x \\
& =\frac{\varepsilon^{\prime 2}}{\varepsilon^{2}}\left\{\int_{x=0}^{\infty} \frac{x}{\left[1+(x \varepsilon)^{2}\right]^{3 / 2}} d x\right. \\
& \left.+2 \int_{x=0}^{\infty} \frac{A}{\left[1+(x \varepsilon)^{2}\right]^{3 / 2}} d x\right\} .
\end{aligned}
$$

Using the inequality

$$
\begin{aligned}
1+\left(x \varepsilon^{\prime}\right)^{2} & \leq\left(1+x \varepsilon^{\prime}\right)^{2} \\
\sqrt{1+\left(x \varepsilon^{\prime}\right)^{2}} & \leq 1+x \varepsilon^{\prime}
\end{aligned}
$$

equation 5.15 is written as

$$
\begin{aligned}
M & \leq \frac{\varepsilon^{\prime 2}}{\varepsilon^{2}}\left\{\int_{x=0}^{\infty} \frac{x}{\left[1+(x \varepsilon)^{2}\right]^{3 / 2}} d x\right. \\
& \left.+2 \int_{x=0}^{\infty} \frac{d x}{\left[1+(x \varepsilon)^{2}\right]^{3 / 2}}+2 \varepsilon^{\prime} \int_{x=0}^{\infty} \frac{x}{\left[1+(x \varepsilon)^{2}\right]^{3 / 2}} d x\right\} .
\end{aligned}
$$

By means of Macsyma [1992], the first integral of (5.17) was found equal to $1 / \varepsilon^{2}$, whereas the second was $1 / \varepsilon$. Substituting these values we obtain

$$
M \leq \frac{\varepsilon^{\prime 2}}{\varepsilon^{2}}\left\{\frac{1}{\varepsilon^{2}}+\frac{2}{\varepsilon}+\frac{2 \varepsilon^{\prime}}{\varepsilon^{2}}\right\} .
$$

Hence

$$
I_{j} \leq I_{0} \leq M \leq f\left(\varepsilon, \varepsilon^{\prime}\right),
$$


or, for non zero principal integral scales all the integrals $I_{j}$ are bounded above.

We proceed now to bound the surface integral $\mathcal{S}(a)$ and to that end (4.109) is written as

$$
\mathcal{S}(a)=-e^{-\rho}+\sum_{j=-\infty}^{+\infty} I_{j}^{\prime},
$$

where

$$
\begin{aligned}
I_{j}^{\prime}= & \frac{\rho \varepsilon_{2} \varepsilon_{3}}{2 \pi} \int_{\theta=0}^{2 \pi} \int_{x=0}^{\infty} \frac{x^{2}}{\left[1+(x R)^{2}\right]^{3 / 2}} \\
& {\left[1+\rho \sqrt{1+(x R)^{2}}\right] e^{-\rho \sqrt{1+(x R)^{2}}} e^{-\rho|1-2 j| x} d x d \theta . }
\end{aligned}
$$

From the inequality $|1-2 j| \geq 1$ it follows that, $I_{j}^{\prime} \leq I_{0}^{\prime}$ for all $j$. Using (5.4), (5.6), and (5.8) (for $\rho_{\chi}=1$ ) one finds that

$$
\begin{aligned}
I_{0}^{\prime \leq} & \rho \varepsilon^{\prime 2} \int_{x=0}^{\infty} \frac{x^{2}}{\left[1+(x \varepsilon)^{2}\right]^{3 / 2}} \\
& {\left[1+\rho \sqrt{1+\left(x \varepsilon^{\prime}\right)^{2}}\right] e^{-\rho \sqrt{1+(x \varepsilon)^{2}}} e^{-\rho x} d x . }
\end{aligned}
$$

Based on the inequality

$$
\begin{aligned}
1+(x \varepsilon)^{2} & \geq(x \varepsilon)^{2} \\
\left(1+(x \varepsilon)^{2}\right)^{3 / 2} & \geq(x \varepsilon)^{2} \\
\frac{1}{\left[1+(x \varepsilon)^{2}\right]^{3 / 2}} & \leq \frac{1}{(x \varepsilon)^{2}},
\end{aligned}
$$

and the fact that $0 \leq \exp \left[-\rho \sqrt{1+(x \varepsilon)^{2}}\right] \leq 1$, one obtains

$$
\begin{aligned}
\int_{x=0}^{\infty} \frac{x^{2}}{\left[1+(x \varepsilon)^{2}\right]^{3 / 2}} e^{-\rho \sqrt{1+(x \varepsilon)^{2}}} e^{-\rho x} d x & \leq \frac{1}{\varepsilon^{2}} \int_{x=0}^{\infty} e^{-\rho x} d x \\
& =\frac{1}{\varepsilon^{2}} \frac{1}{\rho}
\end{aligned}
$$

Using the inequalities

$$
\begin{aligned}
1+\left(x \varepsilon^{\prime}\right)^{2} & \leq\left(1+x \varepsilon^{\prime}\right)^{2} \\
\sqrt{1+\left(x \varepsilon^{\prime}\right)^{2}} & \leq 1+x \varepsilon^{\prime}
\end{aligned}
$$


and $0 \leq \exp \left[-\rho \sqrt{1+(x \varepsilon)^{2}}\right] \leq \exp (-\rho)$, one obtains

$$
\begin{aligned}
& \int_{x=0}^{\infty} \frac{x^{2}}{\left[1+(x \varepsilon)^{2}\right]^{3 / 2}}\left[\rho \sqrt{1+\left(x \varepsilon^{\prime}\right)^{2}}\right] e^{-\rho \sqrt{1+(x \varepsilon)^{2}}} e^{-\rho x} d x \\
\leq & \rho e^{-\rho}\left\{\int_{x=0}^{\infty} \frac{x^{2} e^{-\rho x}}{\left[1+(x \varepsilon)^{2}\right]^{3 / 2}} d x+\varepsilon^{\prime} \int_{x=0}^{\infty} \frac{x^{3} e^{-\rho x}}{\left[1+(x \varepsilon)^{2}\right]^{3 / 2}} d x\right\} .
\end{aligned}
$$

Based on (5.23) we have that

$$
\int_{x=0}^{\infty} \frac{x^{2} e^{-\rho x}}{\left[1+(x \varepsilon)^{2}\right]^{3 / 2}} d x \leq \frac{1}{\varepsilon^{2}} \int_{x=0}^{\infty} e^{-\rho x} d x=\frac{1}{\varepsilon^{2}} \frac{1}{\rho} .
$$

Using the inequality

$$
\begin{aligned}
1+(x \varepsilon)^{2} & \geq(x \varepsilon)^{2} \\
\left(1+(x \varepsilon)^{2}\right)^{3 / 2} & \geq(x \varepsilon)^{3} \\
\frac{1}{\left[1+(x \varepsilon)^{2}\right]^{3 / 2}} & \leq \frac{1}{(x \varepsilon)^{3}}
\end{aligned}
$$

one obtains

$$
\int_{x=0}^{\infty} \frac{x^{3} e^{-\rho x}}{\left[1+(x \varepsilon)^{2}\right]^{3 / 2}} d x \leq \frac{1}{\varepsilon^{3}} \int_{x=0}^{\infty} e^{-\rho x} d x=\frac{1}{\varepsilon^{3}} \frac{1}{\rho} .
$$

Substituting (5.24), (5.26), (5.27), and (5.29) into (5.22) we find that

$$
\begin{aligned}
I_{j}^{\prime} \leq I_{0}^{\prime} & \leq \rho \varepsilon^{\prime 2}\left\{\frac{1}{\varepsilon^{2}} \frac{1}{\rho}+\rho e^{-\rho}\left[\frac{1}{\rho \varepsilon^{2}}+\varepsilon^{\prime} \frac{1}{\rho \varepsilon^{3}}\right]\right\} \\
& =\frac{\varepsilon^{\prime 2}}{\varepsilon^{2}}+\rho e^{-\rho}\left\{\frac{\varepsilon^{\prime 2}}{\varepsilon^{2}}+\frac{\varepsilon^{\prime 3}}{\varepsilon^{3}}\right\} .
\end{aligned}
$$

One can see immediately that as $\rho \rightarrow 0$, or $\rho \rightarrow \infty$ the upper bound in $(5.30)$ becomes $\varepsilon^{\prime 2} / \varepsilon^{2}$.

For non zero principal integral scales we find that

$$
\begin{array}{r}
\lim _{\rho \rightarrow 0}\left\{\rho \varepsilon_{2} \varepsilon_{3} \frac{x^{2}}{\left[1+(x R)^{2}\right]^{3 / 2}}\left[1+\rho\left|\rho_{\chi}\right| \sqrt{1+(x R)^{2}}\right]\right. \\
\left.e^{-\rho\left|\rho_{\chi}\right| \sqrt{1+(x R)^{2}}} e^{-\rho\left|\rho_{\chi}-2 j\right| x}\right\}=0 .
\end{array}
$$


Invoking the Dominated Convergence Theorem we can interchange in (5.2) and (5.21) the order of limit and integration. Then (5.1) becomes

$$
\mathcal{D}(a)=1
$$

and (5.20) gives

$$
\mathcal{S}(a)=-e^{-\rho}
$$

When $\rho \rightarrow 0,(5.33)$ gives $\mathcal{S}(a)=-1$. Upon substitution into (4.115) we obtain

$$
K_{e}=K_{A}=K_{G} \exp \left(\sigma_{Y}^{2} / 2\right)
$$

the arithmetic mean of hydraulic conductivities, expected theoretically [Neuman and Orr, 1993].

For isotropic media we have $\varepsilon_{2}=\varepsilon_{3}=1, R^{2}=1$, and $\varepsilon=\varepsilon^{\prime}=1$. The upper bounds in (5.18) and (5.30) equal 5, and $1+2 \rho \exp (-\rho)$, respectively. For this case (5.31) holds and hence (5.32)-(5.34) apply also for statistically isotropic media.

\subsection{Boundaries Tend to Infinity}

We have shown in Section 5.1 that the domain and surface integrals are bounded above and hence one can interchange limit and integration. For statistically anisotropic media we could not derive a closed form solution (either analytically or via Macsyma [1992]) by taking the limit of the expression within braces in (5.31) as $\rho \rightarrow \infty$. However, when the planes tend to infinity, the surface integral can be seen directly from (4.2) to approach zero. Hence only the domain integral contributes, and the analysis for the effective hydraulic conductivity in infinite domains is provided in Section 3.3. 
We proceed now to evaluate this limit for statistically isotropic media. When $a \rightarrow \infty$ (or $\rho \rightarrow \infty$ ), the Green's function $G_{d}$ and its derivatives are evaluated in the second integral of (3.25) at a point $|\boldsymbol{\chi}| \rightarrow \infty$ and hence, they equal zero. Thus, in (4.114) and (4.115) the surface integral $\mathcal{S}$ vanishes and only $\mathcal{D}$ needs to be taken into account.

When the planes are far from each other, only the index $j=0$ in the infinite series of (4.113) applies (see page 72). Taking the limit of (4.113) as $\rho \rightarrow \infty\left(\lambda_{1} \neq 0\right)$ we obtain

$$
\mathcal{D}=1-\frac{1}{2} \int_{x=0}^{\infty} \frac{x^{2}}{\left(1+x^{2}\right)^{3 / 2}} \mathcal{K} d x
$$

where

$$
\begin{aligned}
\mathcal{K}= & \lim _{\rho \rightarrow \infty} \rho \int_{\rho_{\chi}=-1}^{\rho_{\chi}=+1}\left[1+\rho\left|\rho_{\chi}\right| \sqrt{1+x^{2}}\right] \\
& e^{-\rho\left|\rho_{\chi}\right| \sqrt{1+x^{2}}} e^{-\rho\left|\rho_{\chi}\right| x} d \rho_{\chi} .
\end{aligned}
$$

Upon setting

$$
\begin{gathered}
z=\rho \rho_{\chi}, \\
A=\sqrt{1+x^{2}}
\end{gathered}
$$

we obtain

$$
\mathcal{K}=\lim _{\rho \rightarrow \infty} \int_{z=-\rho}^{z=+\rho}(1+A|z|) e^{-A|z|} e^{-x|z|} d z .
$$

Since the integrand is absolutely convergent (due to the exponential terms) we can write (5.39) as

$$
\mathcal{K}=\int_{z=-\infty}^{z=+\infty}(1+A|z|) e^{-(A+x)|z|} d z .
$$

Upon substitution of the absolute value of $z$ with its appropriate expression, (5.40) becomes

$$
\mathcal{K}=\int_{z=0}^{z=+\infty}(1+A z) e^{-(A+x) z} d z
$$




$$
\begin{aligned}
& +\int_{z=-\infty}^{z=0}(1-A z) e^{(A+x) z} d z \\
& =2\left\{\frac{1}{A+x}+\frac{A}{(A+x)^{2}}\right\},
\end{aligned}
$$

where details of the simple integrations that produced the last result have been omitted. Substituting (5.41) into (5.35) we obtain

$$
\begin{aligned}
\mathcal{D} & =1-\int_{x=0}^{\infty} \frac{x^{2}}{\left(1+x^{2}\right)^{3 / 2}} \frac{1}{A+x} d x \\
& -\int_{x=0}^{\infty} \frac{x^{2}}{\left(1+x^{2}\right)^{3 / 2}} \frac{A}{(A+x)^{2}} d x .
\end{aligned}
$$

Upon replacing the value of $A$ the first integral of (5.42) becomes

$$
\begin{aligned}
I I_{1} & =\int_{x=0}^{\infty} \frac{x^{2}}{\left(1+x^{2}\right)^{3 / 2}} \frac{1}{x+\sqrt{1+x^{2}}} d x \\
& =-\int_{x=0}^{\infty} \frac{x^{2}}{\left(1+x^{2}\right)^{3 / 2}}\left(x-\sqrt{1+x^{2}}\right) d x \\
& =-\int_{x=0}^{\infty} \frac{x^{3}}{\left(1+x^{2}\right)^{3 / 2}} d x+\int_{x=0}^{\infty} \frac{x^{2}}{1+x^{2}} d x
\end{aligned}
$$

The second integral of (5.42) is given by

$$
\begin{aligned}
I_{2} & =\int_{x=0}^{\infty} \frac{x^{2}}{\left(1+x^{2}\right)^{3 / 2}} \frac{\sqrt{1+x^{2}}}{\left(x+\sqrt{1+x^{2}}\right)^{2}} d x \\
& =\int_{x=0}^{\infty} \frac{x^{2}}{1+x^{2}}\left(x-\sqrt{1+x^{2}}\right)^{2} d x \\
& =\int_{x=0}^{\infty} \frac{x^{4}}{1+x^{2}} d x+\int_{x=0}^{\infty} x^{2} d x-2 \int_{x=0}^{\infty} \frac{x^{3}}{\sqrt{1+x^{2}}} d x .
\end{aligned}
$$

Adding up (5.43) and (5.44) we obtain

$$
\begin{aligned}
I I_{1}+I I_{2} & =2 \int_{x=0}^{\infty} x^{2} d x-2 \int_{x=0}^{\infty} \frac{x^{3}}{\sqrt{1+x^{2}}} d x \\
& -\int_{x=0}^{\infty} \frac{x^{3}}{\left(1+x^{2}\right)^{3 / 2}} d x \\
& =\lim _{d \rightarrow \infty}\left\{2 \int_{x=0}^{d} x^{2} d x-2 \int_{x=0}^{d} \frac{x^{3}}{\sqrt{1+x^{2}}} d x\right. \\
& \left.-\int_{x=0}^{d} \frac{x^{3}}{\left(1+x^{2}\right)^{3 / 2}} d x\right\} .
\end{aligned}
$$


From Gradshteyn and Ryzhik [1980, p. 83[8]] we have that

$$
-\int_{x=0}^{d} \frac{x^{3}}{\left(1+x^{2}\right)^{3 / 2}} d x=2-\frac{d^{2}+2}{\sqrt{1+d^{2}}}
$$

whereas the second integral within braces in (5.45) is given by [Gradshteyn and Ryzhik, 1980, p. 83[4]]

$$
-2 \int_{x=0}^{d} \frac{x^{3}}{\sqrt{1+x^{2}}} d x=-\frac{2}{3}\left(d^{2}-2\right) \sqrt{1+d^{2}}-\frac{4}{3} .
$$

Substituting (5.46) and (5.47) into (5.45) we obtain

$$
\begin{aligned}
I I_{1}+I I_{2}= & \lim _{d \rightarrow \infty}\left\{\frac{2 d^{3}}{3}+2-\frac{d^{2}+2}{\sqrt{1+d^{2}}}\right. \\
& \left.-\frac{4}{3}-\frac{2}{3}\left(d^{2}-2\right) \sqrt{1+d^{2}}\right\} .
\end{aligned}
$$

After some algebraic manipulations (5.48) yields

$$
\begin{aligned}
I I_{1}+I I_{2} & =\frac{2}{3}+\lim _{d \rightarrow \infty}\left\{\frac{2 d^{3}}{3}-\frac{d^{2}+2}{\sqrt{1+d^{2}}}-\frac{2}{3}\left(d^{2}-2\right) \sqrt{1+d^{2}}\right\} \\
& =\frac{2}{3}+\lim _{d \rightarrow \infty} \frac{2 d^{3} \sqrt{1+d^{2}}-d^{2}-2 d^{4}-2}{3 \sqrt{1+d^{2}}} \\
& =\frac{2}{3}
\end{aligned}
$$

where the last result was obtained by means of Macsyma [1992]. Upon substitution of (5.49) into (5.42) we obtain

$$
\mathcal{D}=\frac{1}{3}
$$

Equations (4.114) and (4.115) by means of (5.50) become

$$
\begin{aligned}
& K_{e}^{L}=K_{G}\left(1+\frac{\sigma_{Y}^{2}}{6}\right), \\
& K_{e}=K_{G} \exp \left(\sigma_{Y}^{2} / 6\right),
\end{aligned}
$$


respectively. These are the linearized and generalized expressions for the effective hydraulic conductivity in infinite three-dimensional flow domains for statistically isotropic media [Matheron, 1967; Gutjahr et al., 1978; Gelhar and Axness, 1983a; Dagan, 1982a, 1989; Noetinger, 1990]. 


\subsection{Analytical Results for Anisotropic Media}

Let us consider the case where $\varepsilon_{2}=\varepsilon_{3}=\varepsilon=\varepsilon^{\prime} \rightarrow 0$. This corresponds to having mean flow along parallel channels with mutually uncorrelated hydraulic conductivities. For this case we observe that the upper bound in (5.18) tends to infinity and hence we proceed to bound the domain integral in a different way.

The following relationships hold,

$$
\frac{x^{2}}{\left[1+(x R)^{2}\right]^{3 / 2}} \leq x^{2},
$$

since $1+(x R)^{2} \geq 1$

$$
\begin{aligned}
1+\rho\left|\rho_{\chi}\right| \sqrt{1+(x R)^{2}} & \leq 1+\rho \sqrt{1+(x R)^{2}} \\
& \leq 1+\rho(1+x R)=1+\rho+\rho x R
\end{aligned}
$$

because $\left|\rho_{\chi}\right| \leq 1$ and $1+(x R)^{2} \leq(1+x R)^{2}$, and finally,

$$
\exp \left(-\rho\left|\rho_{\chi}\right| \sqrt{1+(x R)^{2}}\right) \leq \exp \left(-\rho\left|\rho_{\chi}\right|\right)
$$

since $\rho\left|\rho_{\chi}\right| \sqrt{1+(x R)^{2}} \geq \rho\left|\rho_{\chi}\right|$.

By virtue of (5.53)-(5.55) we have that the integral $I_{0}$ in (5.2) satisfies

$$
\begin{aligned}
I_{0} \leq & \frac{\rho \varepsilon_{2} \varepsilon_{3}}{4 \pi} \int_{\rho_{\chi}=-1}^{\rho_{\chi}=+1} \int_{\theta=0}^{2 \pi} \int_{x=0}^{\infty} x^{2}(1+\rho+\rho x R) \\
& e^{-\rho\left|\rho_{\chi}\right|(1+x)} d x d \theta d \rho_{\chi} \\
= & \frac{\rho(1+\rho) \varepsilon_{2} \varepsilon_{3}}{2} \int_{\rho_{\chi}=-1}^{\rho_{\chi}=+1} \int_{x=0}^{\infty} x^{2} e^{-\rho\left|\rho_{\chi}\right|(1+x)} d x d \rho_{\chi} \\
+ & \frac{\rho^{2} \varepsilon_{2} \varepsilon_{3}}{4 \pi} \int_{\rho_{\chi}=-1}^{\rho_{\chi}=+1} \int_{\theta=0}^{2 \pi} \int_{x=0}^{\infty} R x^{3} e^{-\rho\left|\rho_{\chi}\right|(1+x)} d x d \theta d \rho_{\chi} .
\end{aligned}
$$

The integral over $\rho_{\chi}$ was calculated with Macsyma [1992]

$$
\int_{\rho_{\chi}=-1}^{\rho_{\chi}=+1} e^{-\rho\left|\rho_{\chi}\right|(1+x)} d \rho_{\chi}=2\left\{\frac{1}{\rho(x+1)}-\frac{e^{-\rho x}}{\rho e^{\rho}(x+1)}\right\}
$$




$$
\leq \frac{2}{\rho(x+1)}
$$

Substituting (5.57) into (5.56) and taking into account that $x^{2} /(x+1) \leq x$ and $x^{3} /(x+1) \leq x^{2}$ we obtain

$$
\begin{aligned}
I_{0} \leq & (1+\rho) \varepsilon_{2} \varepsilon_{3} \int_{x=0}^{\infty} x d x \\
& +\frac{\rho \varepsilon_{2} \varepsilon_{3}}{2 \pi} \int_{\theta=0}^{2 \pi} R \int_{x=0}^{\infty} x^{2} d x d \theta
\end{aligned}
$$

Setting $R=\varepsilon$ the integral over $\theta$ equals $2 \pi \varepsilon$. Substituting the upper limit of the integral over $x$ with $1 / \varepsilon($ where $\varepsilon \rightarrow 0)$ we obtain

$$
I_{0} \leq(1+\rho) \varepsilon^{2} \frac{1}{2 \varepsilon^{2}}+\rho \varepsilon^{3} \frac{1}{3 \varepsilon^{3}}=\frac{5 \rho+3}{6} .
$$

Hence for finite $\rho$, integral $I_{0}$ and consequently $I_{j}$ (for all $j$ ) is bounded above by a constant. For the surface integral the bound in (5.30) equals $1+2 \rho \exp (-\rho)$.

Upon substitution of $\varepsilon_{2}=\varepsilon_{3}=\varepsilon$ the limit as $\varepsilon \rightarrow 0$ of the expression within braces in (5.31) is found to equal zero [Macsyma, 1992]. Invoking the Dominated Convergence Theorem we interchange the order of limit and integration to obtain

$$
\begin{gathered}
\mathcal{D}(a)=1, \\
\mathcal{S}(a)=-e^{-\rho} .
\end{gathered}
$$

Substituting (5.60) and (5.61) into (4.115) we finally obtain

$$
K_{e}(a)=K_{G} \exp \left\{\sigma_{Y}^{2}[\exp (-\rho)-0.5]\right\}
$$

which is shown in Figure 5.1. When $\rho \rightarrow 0$ and $\rho \rightarrow \infty$ we obtain the arithmetic and harmonic mean of hydraulic conductivities, respectively. When $\varepsilon_{2}=\varepsilon_{3}=\varepsilon=\varepsilon^{\prime} \rightarrow$ $\infty$ the bound in (5.18) tends to zero, and the bound of (5.30) equals $1+2 \rho \exp (-\rho)$. Hence, we obtain again (5.62). 


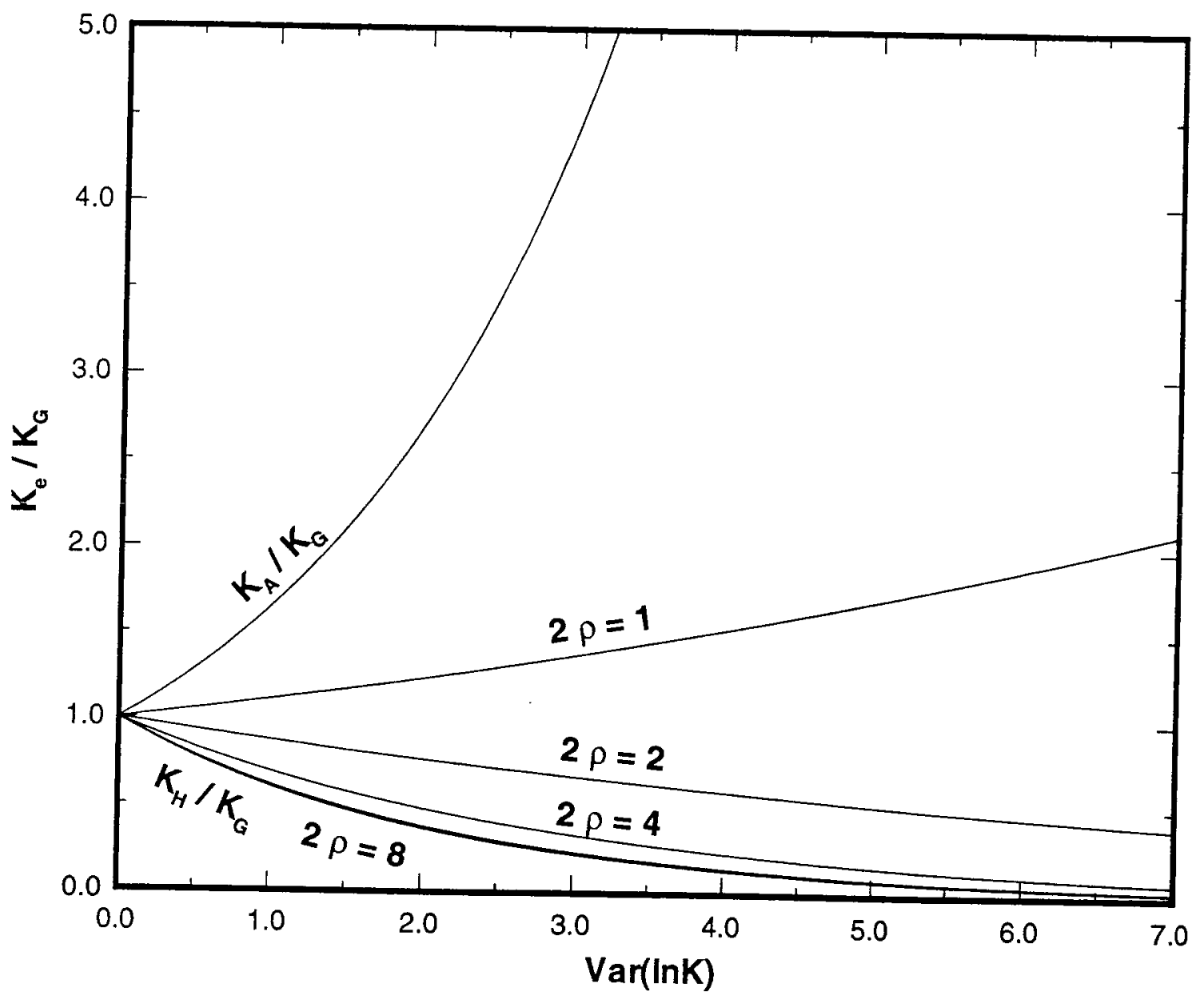

Figure 5.1: Normalized $K_{e}$, equation 5.62, as a function of $\sigma_{Y}^{2}$ and $2 \rho$. 


\subsection{Numerical Results for Isotropic Media}

We present below numerical results for the linearized form of $K_{e}$ in (4.114) and the generalized form in (4.115) in statistically isotropic media.

Our results were calculated with the program "Plates.For" (Appendix Q) which relies on Gaussian quadrature (Appendix K) for the evaluation of (4.110) and (4.113). Although such a numerical scheme is appropriate only for finite intervals (the integral on $x$ in (4.110) and (4.113) has an infinite upper limit), the negative exponentials in these expressions dominate at large values of $x$ so that both the surface and the domain integrands decay rapidly with $x$. Figures 5.2 to 5.10 show the behavior and magnitude of the integrands of (4.110) and (4.113) for different values of $\rho$ and $j$. In the plots of the domain integrands the dimensionless length $\rho_{\chi}$ is shown as $u$.

From these figures, for each $\rho$ and $j$, one can approximately evaluate the area and volume under the curves and hence, obtain an idea of the magnitude of the integrals of (4.110) and (4.113). Multiplying the area by $\rho$, and the volume by $\rho / 2$, one can approximately calculate $I_{j}^{\prime}$ in (5.21) and $I_{j}$ in (5.2), respectively (for $\varepsilon_{2}=\varepsilon_{3}=1$ ). For statistically isotropic media we have found that the upper bounds for $I_{j}$ and $I_{j}^{\prime}$ are 5 and $1+2 \rho \exp (-\rho)$, respectively (Section 5.1). Figures 5.2, 5.3, $5.5,5.7$, and 5.9 show that $I_{j}^{\prime} \leq I_{0}^{\prime} \ll 1 \leq 1+2 \rho \exp (-\rho)$. From the plots of the domain integrands one can easily see that $I_{j} \leq I_{0} \leq 5$. For example, for $\rho=0.01$ and $j=0$ (Figure 5.10 ), one can calculate an area of a triangle $0.3 \times 100 / 2$, multiply it by 2 , and obtain a volume of approximately 30 units. Multiplying by $0.01 / 2$ the value of $I_{0}$ is approximately $0.15 \ll 5$. Hence, by means of Figures 5.2 to 5.10 one can verify the bounds of Section 5.1. Indeed, our numerical results show that $I_{j}$ and 
$I_{j}^{\prime}$ decrease in magnitude with increasing $|j|$, and for all $\rho$ and $j$ the above bounds are satisfied.

The numerical procedure for the evaluation of (4.110) and (4.113) was as follows:

1. The number of Gauss points required for accurate evaluation of (4.110) and (4.113) was established via numerical experiments, initially for the case where the boundaries are far apart, and subsequently for the bounded cases. Thus, for the domain integral in (4.113) 1000 points were used within each interval in the $x$-direction, and 360 in the $u$ - (or $\rho_{\chi^{-}}$) direction. The higher number of Gauss points in the $x$ - than the $u$-direction was required in order to capture the sharp behavior of the domain integrand at low values of $x$. For the surface integral in (4.110) which has a much smoother behavior, we used 360 points in the $x$-direction.

2. The terms of the infinite series that have a significant contribution to (4.110) and (4.113) were established. It can be easily seen that inequality $\left|\rho_{\chi}-2 m\right| \geq$ $\left|\rho_{\chi}-2 n\right|$ holds for all $|m| \geq|n|$, where $m, n$ take values from $-\infty$ to $+\infty$. From this it follows that $\exp \left(-\rho\left|\rho_{\chi}-2 m\right| x\right) \leq \exp \left(-\rho\left|\rho_{\chi}-2 n\right| x\right)(\rho, x \geq 0)$. Hence the terms of the series decrease in magnitude as the index $j$ takes (absolutely) higher values. We initially used Figures 5.2 to 5.10 to get an estimate of the magnitude of the domain and surface integral for different values of $j$. This enabled us identify (for each $\rho$ ) an approximate upper value $k$ of the index $|j|$ and avoid excessive computational effort. Using $k$ as our guideline we retained for each $\rho$ these terms of the series that differ up to 6 orders of magnitude from the $j=0$ term. We give below the number of terms of the series calculated in 
each case:

- For $\rho=50$, Figure 5.2, only the $j=0$ term was evaluated.

- For $\rho=10$, we calculated (4.110) and (4.113) up to the $j= \pm 2$ terms.

- For $\rho=4$, and $\rho=2$, Figures 5.3, and 5.5 show that the surface area for $j=10$ is of the order of $10^{-6}$, and $10^{-5}$, respectively. The volume of the domain integral, Figures 5.4, and 5.6, for $j= \pm 2$, and $j= \pm 4$, respectively, is of the order of $10^{-3}$. The calculations in these cases, as well as for $\rho=1$, Figures 5.7, and 5.8, were performed up to $j= \pm 10$.

- For $\rho=0.01$, Figures 5.9, and 5.10, significant contribution is provided by a large number of terms. Based on the above criterion the evaluation proceeded up to $j= \pm 100$.

3. Finally, the number and length of the discretization intervals (over which the Gaussian quadrature was applied) was established based on the behavior of the domain and surface integrands. In general, a much finer discretization was required for small values of $x$, where the integrand in (4.110) and (4.113) rises sharply (Figures 5.2 to 5.10 ) than for larger values of $x$. In the $x$-direction for both domain and surface integral an interval of 0.2 was used up to $x=5,0.5$ up to $x=20,1$ up to $x=50,2$ up to $x=100$, and 100 up to $x=1000$. In

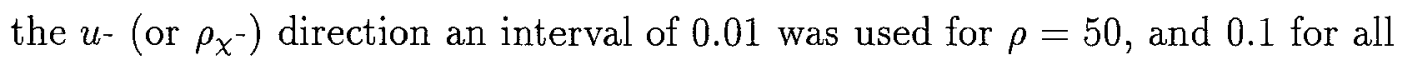
other values of $\rho$.

All calculations were performed on the University of Arizona Convex C240 with four vector processors, except for the computationally intensive case of $\rho=0.01$ which was run on the Connection machine at Los Alamos National Laboratories. Our 
results for the domain integral $\mathcal{D},(4.113)$, and the surface integral $\mathcal{S},(4.110)$, are shown in Table 5.1 for different ratios $2 \rho=2 a / \lambda$ of distance between the Dirichlet boundaries and integral scale. We also show in the same table the values of the normalized effective hydraulic conductivity, $K_{e} / K_{G}$ from (4.115), and the linearized expression $K_{e}^{L} / K_{G},(4.114)$, both for $\sigma_{Y}^{2}=1$.

\begin{tabular}{|l|l|l|l|l|}
\hline Ratio $2 \rho$ & $\mathcal{D}(\rho)$ & $\mathcal{S}(\rho)$ & $K_{e} / K_{G}$ (eq. 4.115) & $K_{e}^{L} / K_{G}$ (eq. 4.114) \\
\hline 0.02 & 0.127487 & -0.127055 & 1.6480 & 1.4996 \\
\hline 2 & 0.206813 & -0.076014 & 1.4466 & 1.3692 \\
\hline 4 & 0.272186 & -0.045962 & 1.3149 & 1.2738 \\
\hline 8 & 0.317110 & -0.009506 & 1.2121 & 1.1924 \\
\hline 20 & 0.320503 & $-0.289 * 10^{-4}$ & 1.1966 & 1.1795 \\
\hline 100 & 0.325204 & $-3.06^{*} 10^{-22}$ & 1.1910 & 1.1748 \\
\hline
\end{tabular}

Table 5.1: Effective Hydraulic Conductivity in Bounded Domains.

From Table 5.1 , for $2 \rho=0.02$, the $\operatorname{sum} \mathcal{D}+\mathcal{S}=4 * 10^{-4}$ is very close to the exact value of $\mathcal{D}+\mathcal{S}=0$ which corresponds to the arithmetic mean $K_{A}=$ $K_{G} \exp \left(\sigma_{Y}^{2} / 2\right)$ expected theoretically as $\rho \rightarrow 0$ (Section 5.1). The normalized $K_{e}^{L} / K_{G}$ takes the value of 1.4996 which differs only by $0.03 \%$ from the linearized value $K_{A}^{L} / K_{G}=1+\sigma_{Y}^{2} / 2$. We tried also to verify analytically the values of $\mathcal{D}$ and $\mathcal{S}$ as $\rho \rightarrow 0.01$. We have shown in Section 5.1 that the domain and surface integrals are bounded above. The limit of the left hand side of (5.31) as $\rho \rightarrow 0.01$ was evaluated by means of Macsyma [1992] and was found to be a function $f\left(x,\left|\rho_{\chi}\right|, j\right)$. Invoking the Dominated Convergence Theorem we interchanged in (4.113) the order of limit and integration. The integral over $\rho_{\chi}$ of $f\left(x,\left|\rho_{\chi}\right|, j\right)$ was calculated by Macsyma [1992]. However, the integral over $x$ of the resulting expression does not appear in any available tables (and could not be evaluated by Macsyma [1992]). 
Our analysis in Section 5.1 and the numerical result at $2 \rho=0.02$ supports the theoretical finding by Neuman and Orr [1993] that $K_{e}$ reduces to $K_{A}$ when the flow domain $\Omega$ shrinks to the support volume $\omega$. It also coincides with the numerical result by Desbarats [1992a] when the ratio of characteristic length of the domain to integral scale approaches the value of 0.1 . Desbarats [1992a] investigated numerically steady state flow in a cubic field with constant head and no-flow boundaries in the longitudinal and transverse directions, respectively. For isotropic media he found that, for the above ratio and $\sigma_{Y}^{2}=1.0$, the sample mean (from 200 realizations) of block-averaged hydraulic conductivities (defined via (2.76)) approaches $K_{A}$.

At $2 \rho=100, \mathcal{D}+\mathcal{S}=0.325204$ differs by only $2 \%$ from the exact theoretical value $\mathcal{D}+\mathcal{S}=1 / 3$ corresponding to the infinite domain solution $K_{\infty}=K_{G} \exp \left(\sigma_{Y}^{2} / 6\right)$ [Noetinger, 1990; Dykaar and Kitanidis, 1992b; Neuman et al. 1992]. The exact infinite domain solution was previously derived in Section 5.2 by formally taking the limit of our expressions as $\rho \rightarrow \infty$. Hence, this $2 \%$ difference need not be entirely due to a numerical error, as a length of 100 integral scales does not necessarily correspond to an infinite domain.

The normalized effective hydraulic conductivity, (4.115), is shown in Figure 5.11 as a function of $\sigma_{Y}^{2}$ for various $2 \rho$. Here $K_{A}=K_{G} \exp \left(\sigma_{Y}^{2} / 2\right)$ and $K_{\infty}=$ $K_{G} \exp \left(\sigma_{Y}^{2} / 6\right)$ designate the arithmetic mean of hydraulic conductivities and the infinite domain solution, respectively. Figure 5.12 presents $K_{e},(4.115)$, as a function of $2 \rho$ for various $\sigma_{Y}^{2}$. The value of $K_{e}$ (for each $\sigma_{Y}^{2}$ ) equals the arithmetic mean, $K_{A}$, at $2 \rho=0$, and approaches asymptotically the infinite domain solution, $K_{\infty}$, at large values of $2 \rho$. The linearized $K_{e}^{L},(4.114)$, is shown in Figure 5.13, with $K_{A}^{L}=K_{G}\left(1+\sigma_{Y}^{2} / 2\right)$ and $K_{\infty}^{L}=K_{G}\left(1+\sigma_{Y}^{2} / 6\right)$. 
The three figures show that, for statistically isotropic media and arbitrarily large $\sigma_{Y}^{2}$, the assumption of an infinite domain is accurate for distances between the two constant head planes exceeding 8 integral scales. At $2 \rho=8$ the sum $\mathcal{D}+$ $\mathcal{S}=0.307604$ differs $8 \%$ from the exact theoretical value $\mathcal{D}+\mathcal{S}=1 / 3$ which corresponds to $K_{\infty}$. Rubin and Dagan [1988] found that the effect of a constant head boundary in a two-dimensional, semi-infinite flow domain, becomes negligible at distances greater than 4 integral scales from this boundary. This coincides with our finding which concerns the effect of two such boundaries. The effect of an impervious boundary was found [Rubin and Dagan, 1989] to be felt within 3 integral scales from the boundary. Desbarats and Dimitrakopoulos [1990] showed numerically that the sample mean of block-averaged transmissivities in a two-dimensional bounded flow domain approaches the infinite domain $K_{e}$ value when the characteristic length of the domain exceeds 20 integral scales. Desbarats [1992a] showed numerically that the sample mean of block-averaged conductivities in a three-dimensional cubic field approaches the infinite domain $K_{e}$ value when the ratio of characteristic domain length to integral scale exceeds 10 . Dykaar and Kitanidis [1992b] found that for small $\sigma_{Y}^{2}$, and large $\sigma_{Y}^{2}$, respectively, 20 and 80 integral scales were needed for boundary effects in 2-D to decay. In 3-D, 10 and 30 integral scales were required.

Smith and Freeze [1979b] found that the infinite domain $K_{e}$ in a twodimensional bounded domain is a very good approximation to the true $K_{e}$. They used $\sigma_{Y}^{2}=0.83$ and ratios $a_{x}$ and $a_{y}$ of distance between the Dirichlet boundaries to integral scale and no-flow boundaries to integral scale, between 8 and 20 , and 4 and 10, respectively. For the range of values of $a_{y}$ of this study the impervious boundaries exert a minor influence on the solution of the effective conductivity [Rubin and 
Dagan, 1989]. In addition, based on our three-dimensional results we ascertain that for such values of $\sigma_{Y}^{2}$ and $a_{x}$ the solution of a bounded domain is practically indistinguishable from that of an infinite domain. Such an agreement would not, in general, hold for larger variances and smaller flow domains (Figures 5.11 and 5.13).

Rubin and Gómez-Hernández [1990] found that the applicability of their analytical approach to two-dimensional bounded domains improved with an increase in the size of the domain. We recall that their equation 2.75 was linearized and also that an infinite expression for the the cross covariance of $Y$ and gradient of head was used in (2.75). Hence a large bounded domain makes the applicability of the above more appropriate (Figures 5.11 and 5.13). Moreover, these authors stated: "this increase in $S$ counterweighted any increase in $\sigma_{Y}^{2} "$. Indeed, from Figures 5.11 and 5.13 one observes that by increasing $\sigma_{Y}^{2}$ the divergence of the bounded domain solution from the infinite one becomes more pronounced. For fixed $\sigma_{Y}^{2}$ an increase in the size of the domain brings the true solution closer to the infinite one. For domains with characteristic length of 6 integral scales and $\sigma_{Y}^{2}$ up to 2 [ibid, Figures 8 and 9] they found a good agreement between their analytical and the sample mean of a numerical solution (which corresponds approximately to an effective parameter). For these values the infinite domain solution constitutes a very good approximation to the true solution (Figures 5.11 and 5.13).

Finally, the behavior of the domain integral $\mathcal{D},(4.113)$, and the surface integral $\mathcal{S},(4.110)$, as a function of $2 \rho$, are shown in Figures 5.14 and 5.15. These figures and Table 5.1 show that, contrary to Dagan's assertion [1989], $\mathcal{S}$ is as important as $\mathcal{D}$ when $2 \rho$ becomes small. At $2 \rho=8,2 \rho=4$, and $2 \rho=2$, respectively, the value of $|\mathcal{S}|$ corresponds to $3 \%, 17 \%$, and $37 \%$ of that by $\mathcal{D}$. When $2 \rho=0.02,|\mathcal{S}|$ becomes 
approximately equal to $\mathcal{D}$.

Dykaar and Kitanidis [1992b], Figure 2.1, presented results for the effective hydraulic conductivity for statistically isotropic media with small $\sigma_{Y}^{2}$ (a value for $\sigma_{Y}^{2}$ is not provided) in three- and two-dimensional flow domains. These were obtained by numerical evaluation of the linearized expression (2.67) over the finite averaging volume of a local element of a global grid. The two- and three-dimensional $K_{e}^{L}$ values were normalized by $K_{G}$ and $K_{\infty}^{L}=K_{G}\left(1+\sigma_{Y}^{2} / 6\right)$, respectively.

Figure 2.1 shows that at a ratio of characteristic length of local element to integral scale, $r$, equal to zero, the $K_{e}^{L}$ values are much higher than the linearized infinite domain solutions. Neuman and Orr [1993] have shown that when the flow domain $\Omega$ shrinks to a point (practically the support volume $\omega$ ) then $K_{e}$ reduces to $K_{A}$. We inferred the value of $\sigma_{Y}^{2}=0.95$ as the value that, upon substitution in the three- and two-dimensional normalized expressions $K_{A}^{L} / K_{\infty}^{L}$ and $K_{A}^{L} / K_{G}$, would reproduce the values 1.27 and 1.475 , respectively, of the normalized $K_{e}^{L}$ of Figure 2.1 at $r=0$.

Values of the three-dimensional $K_{e}^{L} / K_{\infty}^{L}$ for different ratios $r$ for the isotropic exponential covariance function were obtained directly from Figure 2.1 and are listed in the second column of Table 5.2. The $K_{e}^{L} / K_{G}$ values of Dykaar and Kitanidis [1992] are listed in column 3 of Table 5.2. They were calculated by multiplying the normalized results of column 2 , Table 5.2 , by the constant $\left(1+\sigma_{Y}^{2} / 6\right)=1.1583$ corresponding to $\sigma_{Y}^{2}=0.95$. Column 4 gives our $K_{e}^{L} / K_{G}$ values, (4.114), that correspond to $\sigma_{Y}^{2}=0.95$. Column 5 compares the values in column 3 with those in column 4. 


\begin{tabular}{|l|l|l|l|l|l|}
\hline \multirow{2}{*}{ Ratio $r$} & \multicolumn{2}{l|}{ Method of Moments } & Residual Flux & \% Diff. & Value of $F$ \\
\cline { 2 - 5 } & $K_{e}^{L} / K_{\infty}^{L}$ & $K_{e}^{L} / K_{G}$ & $K_{e}^{L} / K_{G}$ & & \\
\hline 0.02 & 1.270 & 1.471 & 1.4746 & 0.2 & 0.004 \\
\hline 2 & 1.100 & 1.274 & 1.3507 & 5.7 & 0.212 \\
\hline 4 & 1.050 & 1.216 & 1.2601 & 3.5 & 0.273 \\
\hline 8 & 1.015 & 1.176 & 1.1828 & 0.6 & 0.315 \\
\hline
\end{tabular}

Table 5.2: $K_{e}$ values in bounded domains: method of moments and residual flux theory.

Equation (2.67) for the isotropic case can be rewritten as

$$
K^{e}=K_{G}\left\{1+\sigma_{Y}^{2}\left(\frac{1}{2}-F\right)\right\} \quad \text { with } \quad F=\sum_{\boldsymbol{m}} \frac{m_{i}^{2} \mathcal{Q} \boldsymbol{m}}{\boldsymbol{m}^{2}},
$$

where $\mathcal{Q m}_{\boldsymbol{m}}$ is the spectral density of $Y$. Equation 5.63 can be solved for $F$ using the values in column 3 of Table 5.2. Values of $F$ are listed in column 6 of Table 5.2, for various $r$. The values of 0.004 and 0.334 , corresponding to $r=0.02$ and $r=10$, respectively, reproduce $K_{A}^{L}$ and $K_{\infty}^{L}$. The fact that the solution for $K_{e}$, derived via the method of moments for periodic media, provides correct limit behavior at $r=0$ indicates that it may be capturing boundary effects. Comparing the values of $F$ in column 6 of Table 5.2 with those of $\mathcal{D}$ in column 2 of Table 5.1 for $2 \rho=r=2,4,8$, shows that $F=\mathcal{D}$ to a high degree of accuracy. 

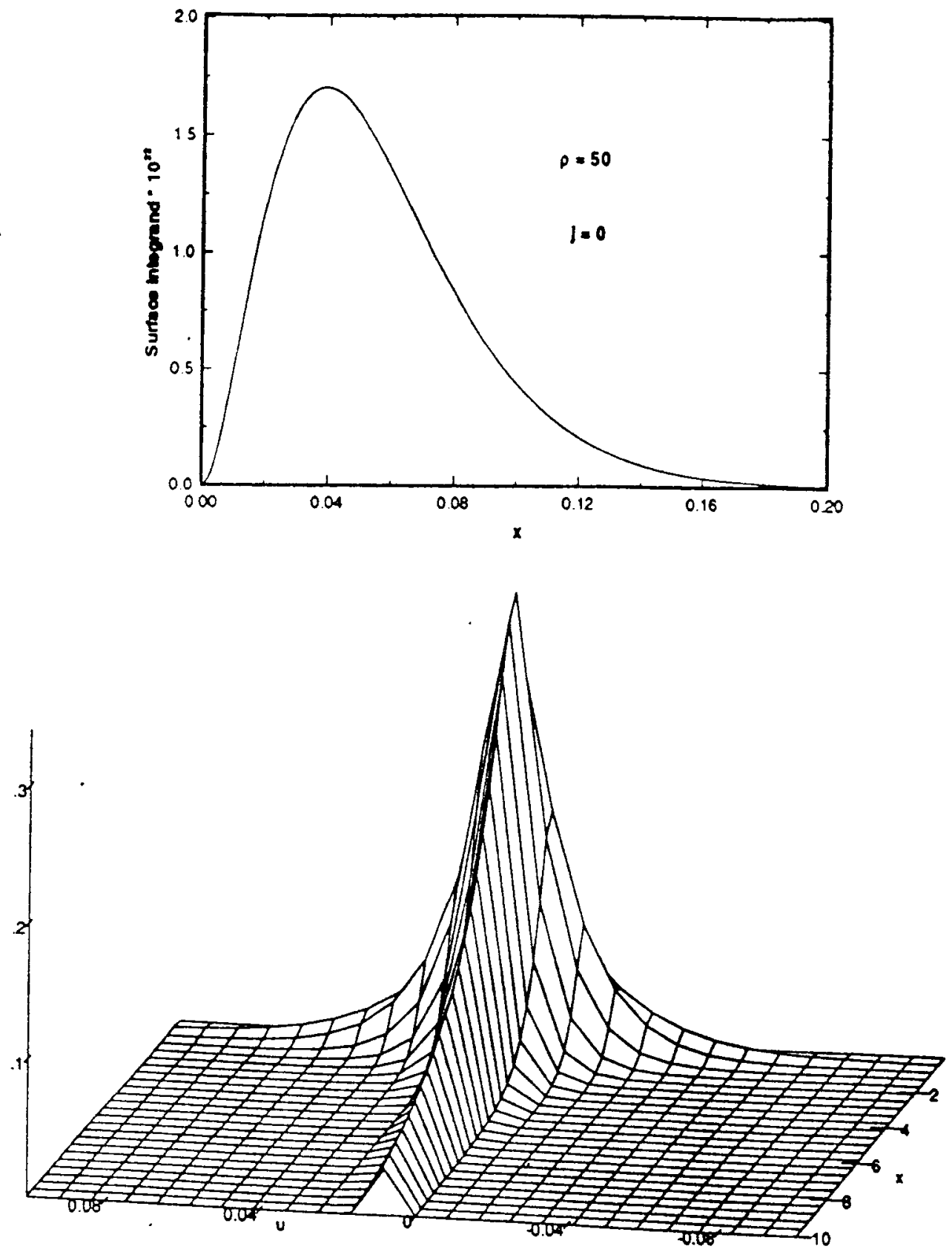

Figure 5.2: Surface and domain integrands, (4.110) and (4.113), $\rho=50$ and $j=0$. 

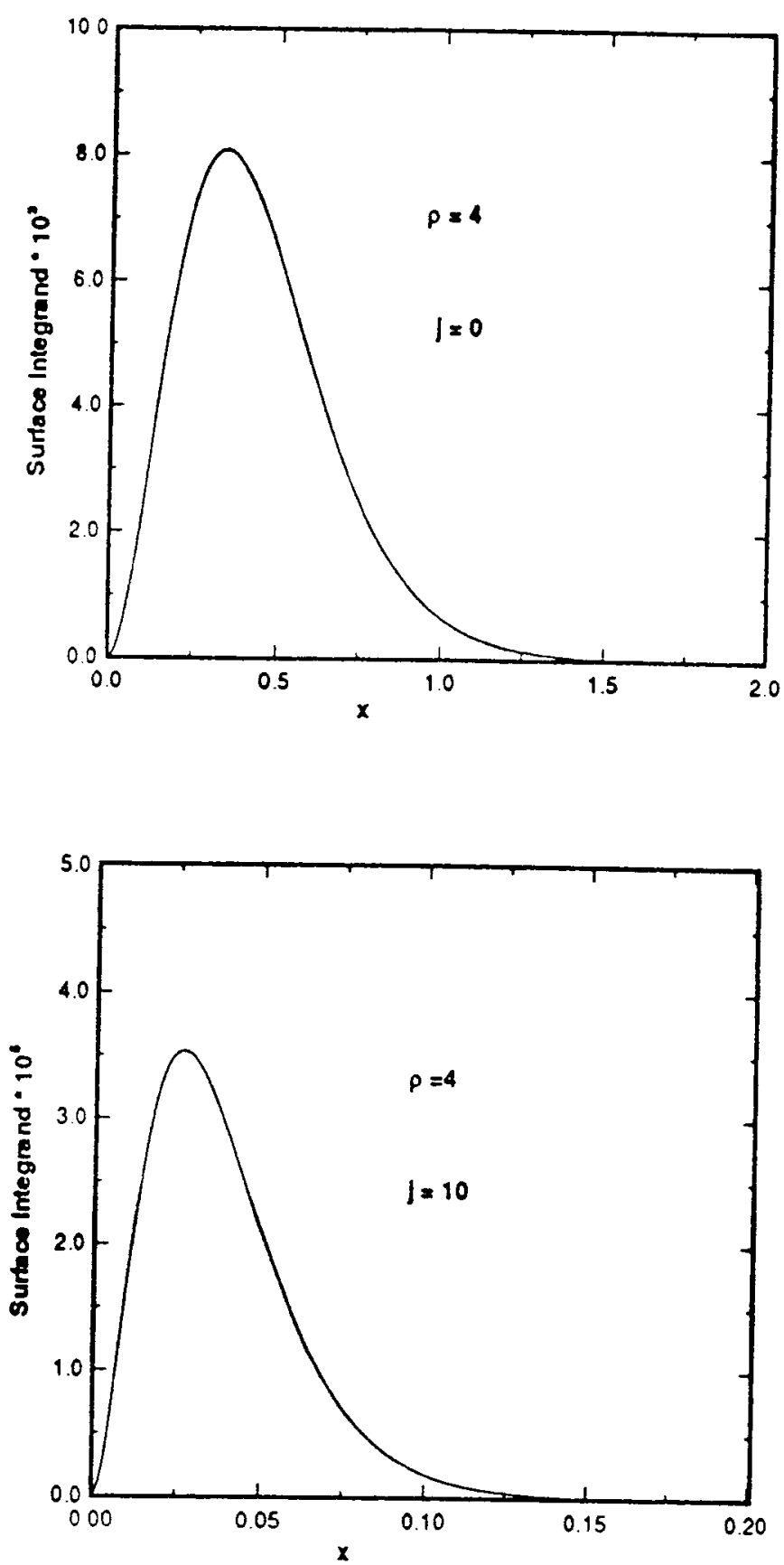

Figure 5.3: Surface integrand, (4.110), $\rho=4, j=0$, and $j=10$. 

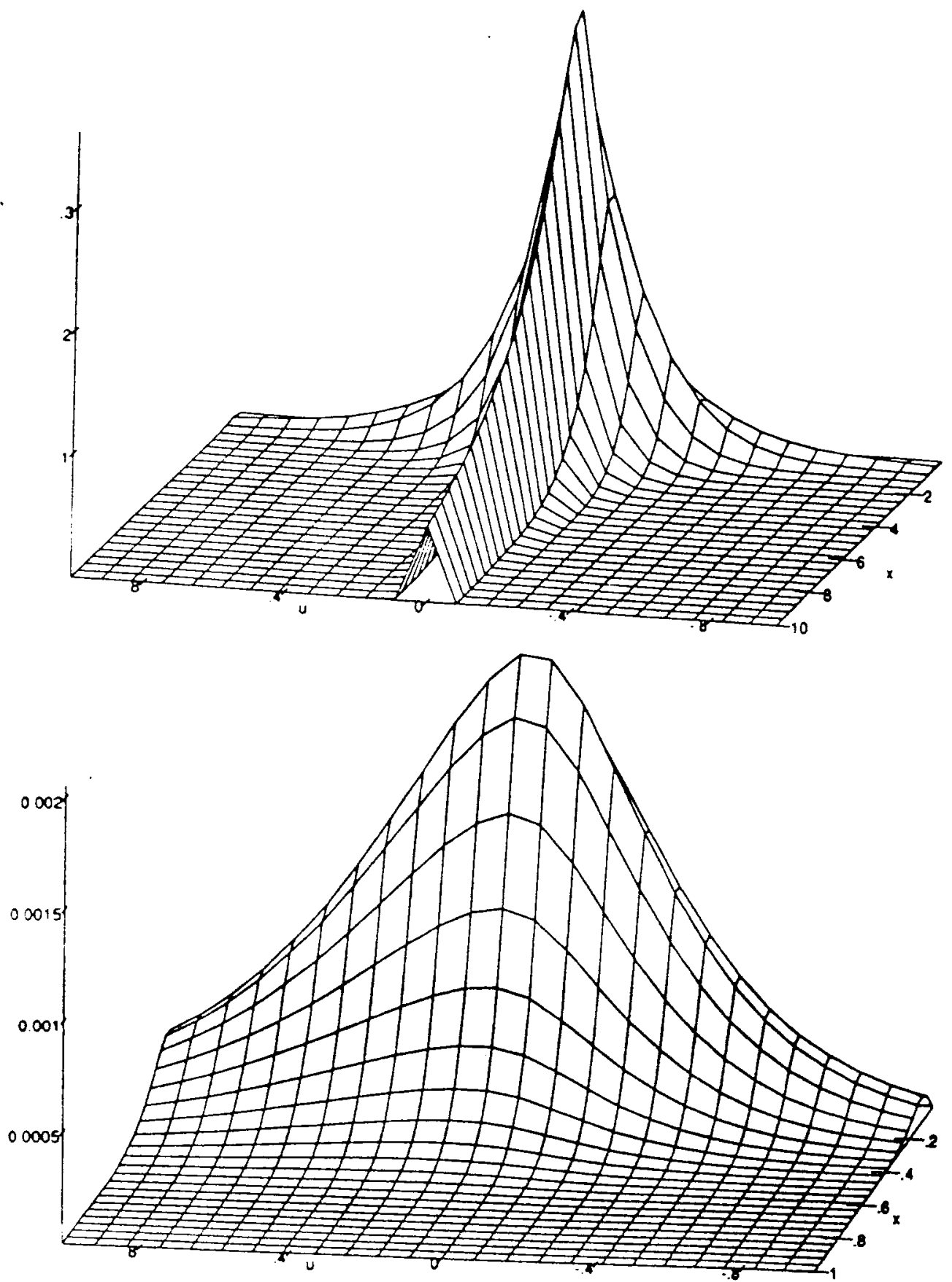

Figure 5.4: Domain integrand, (4.113), $\rho=4, j=0$ (top), and $j= \pm 2$. 

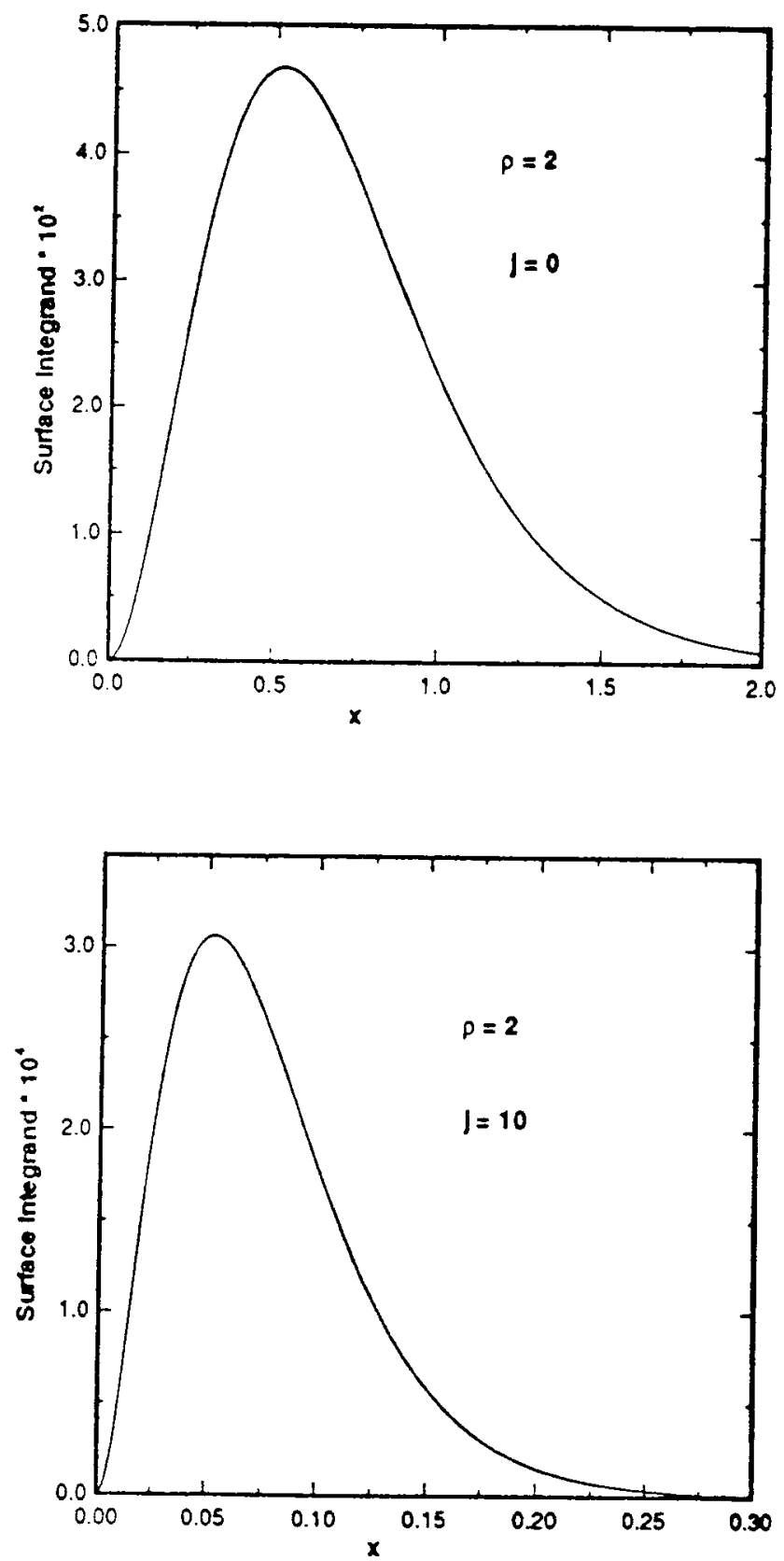

Figure 5.5: Surface integrand, (4.110), $\rho=2, j=0$, and $j=10$. 

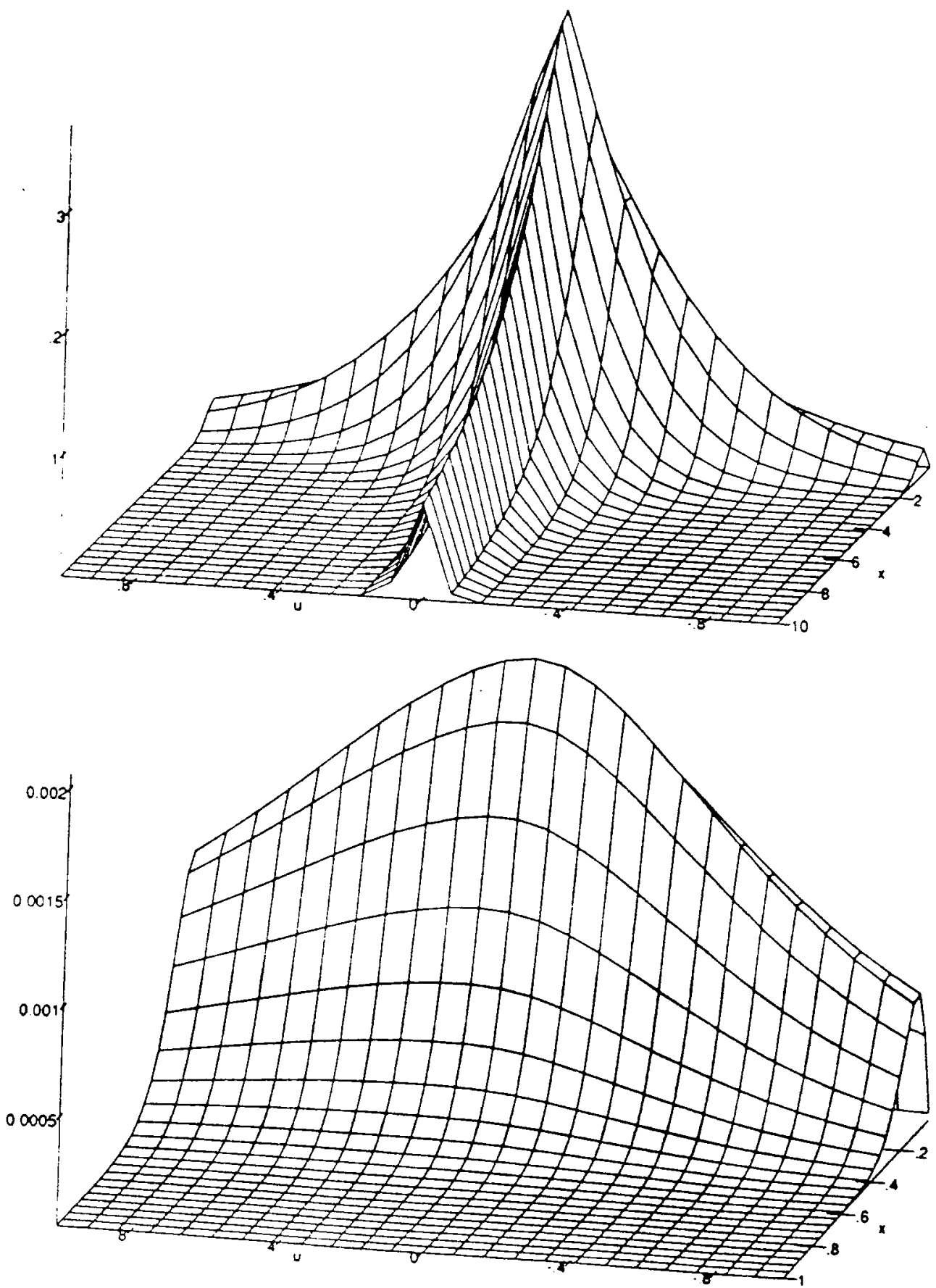

Figure 5.6: Domain integrand, (4.113), $\rho=2, j=0$ (top), and $j= \pm 4$. 

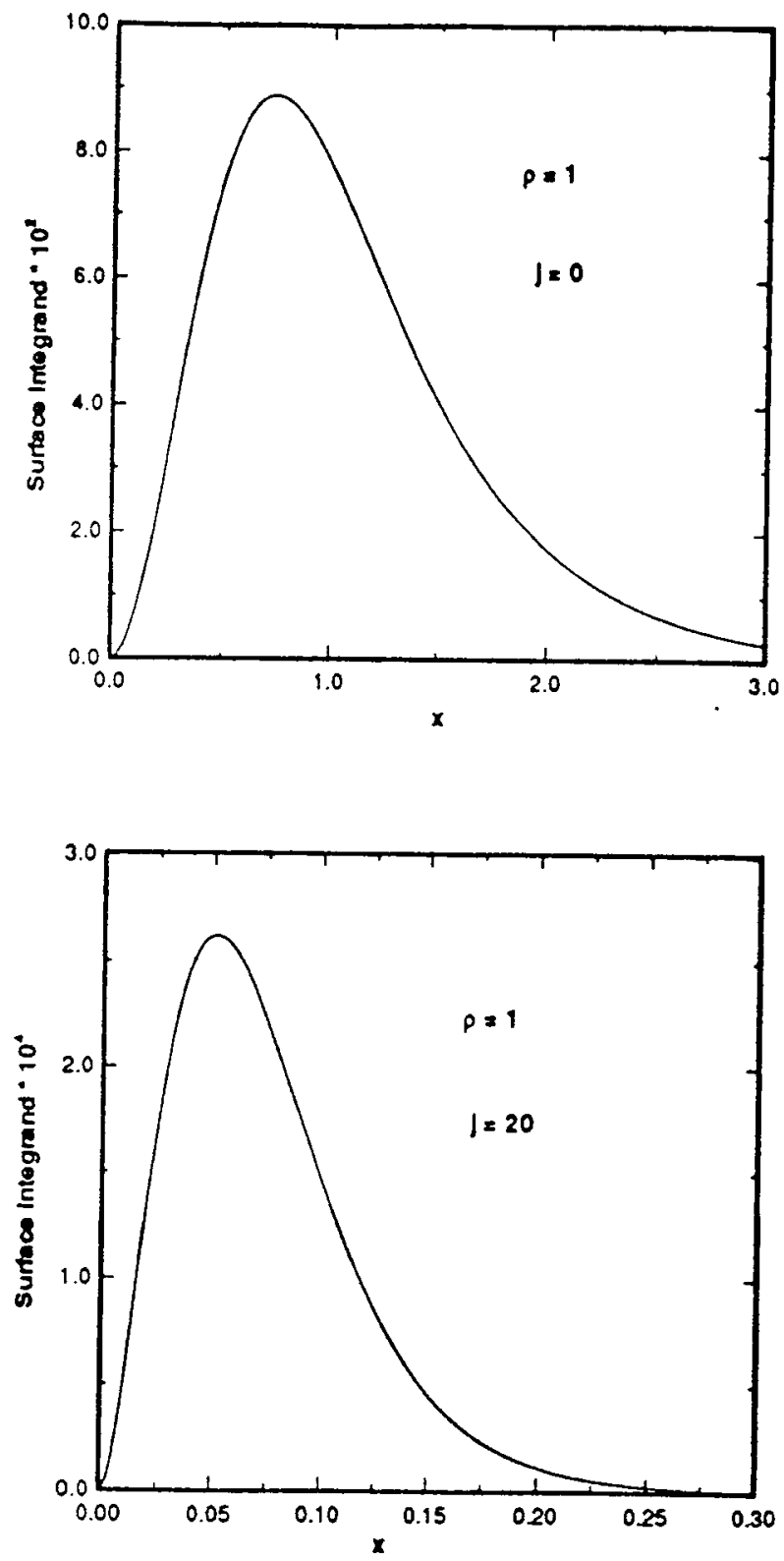

Figure 5.7: Surface integrand, (4.110), $\rho=1, j=0$, and $j=20$. 

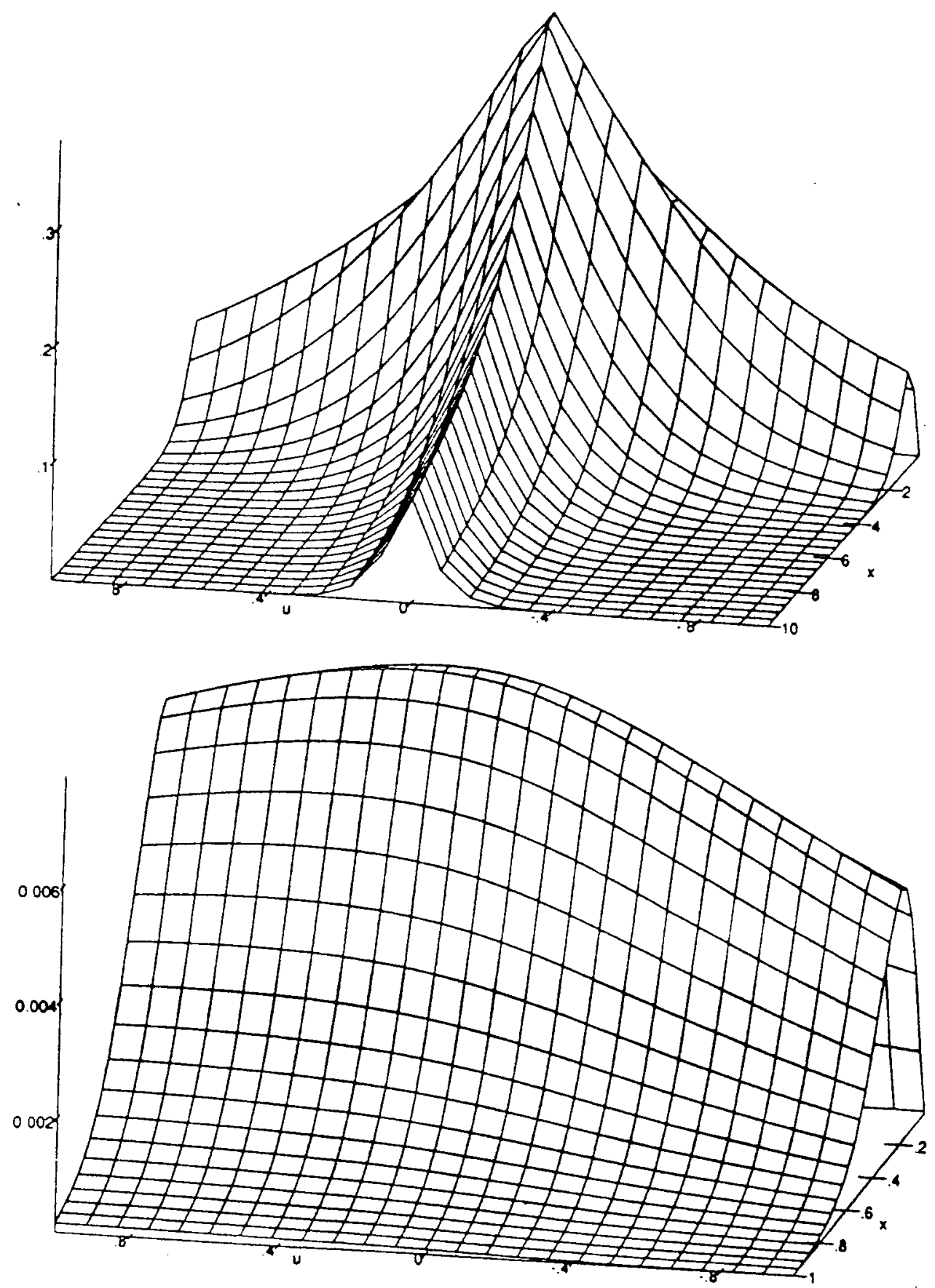

Figure 5.8: Domain integrand, (4.113), $\rho=1, j=0$ (top), and $j= \pm 4$. 

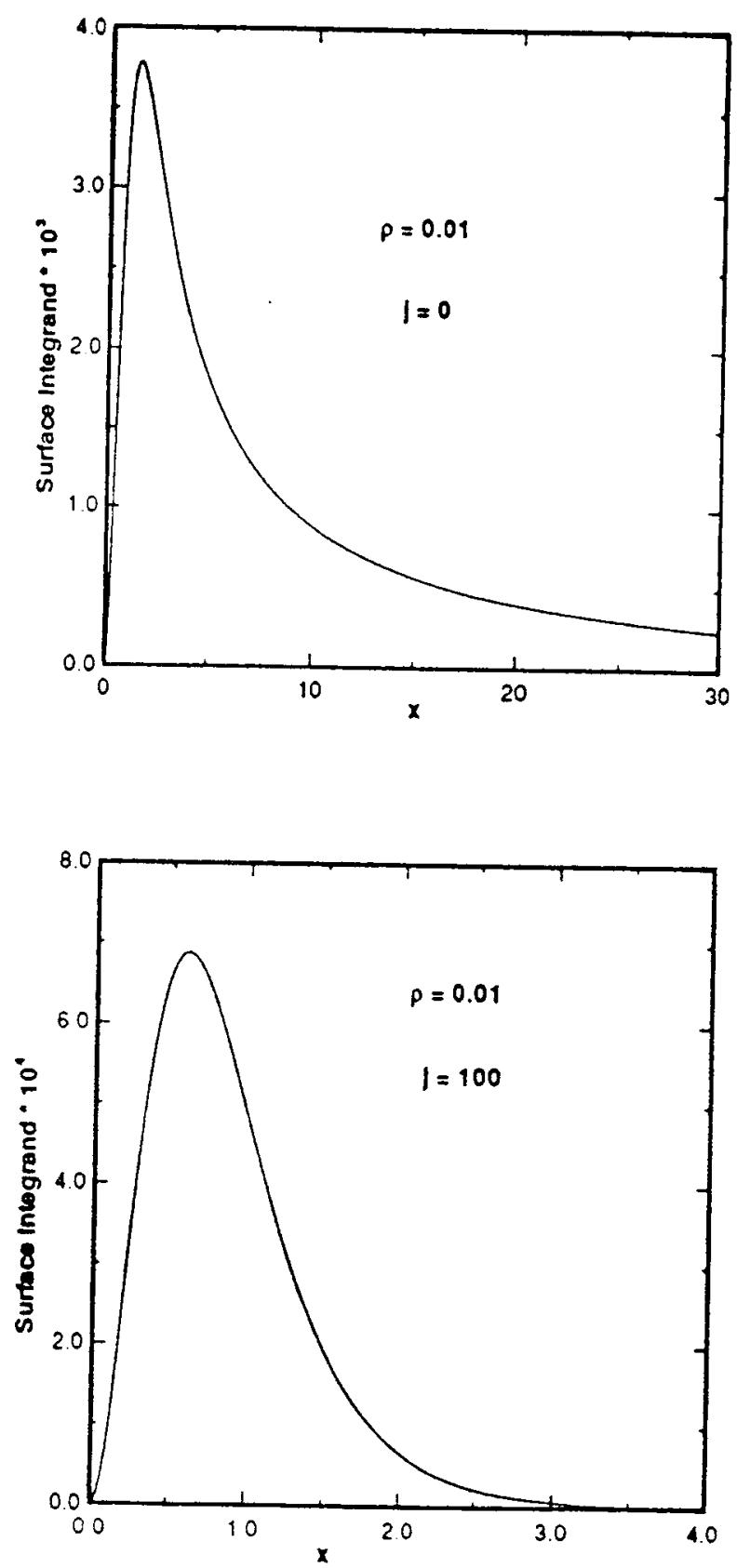

Figure 5.9: Surface integrand, (4.110), $\rho=0.01, j=0$, and $j=100$. 

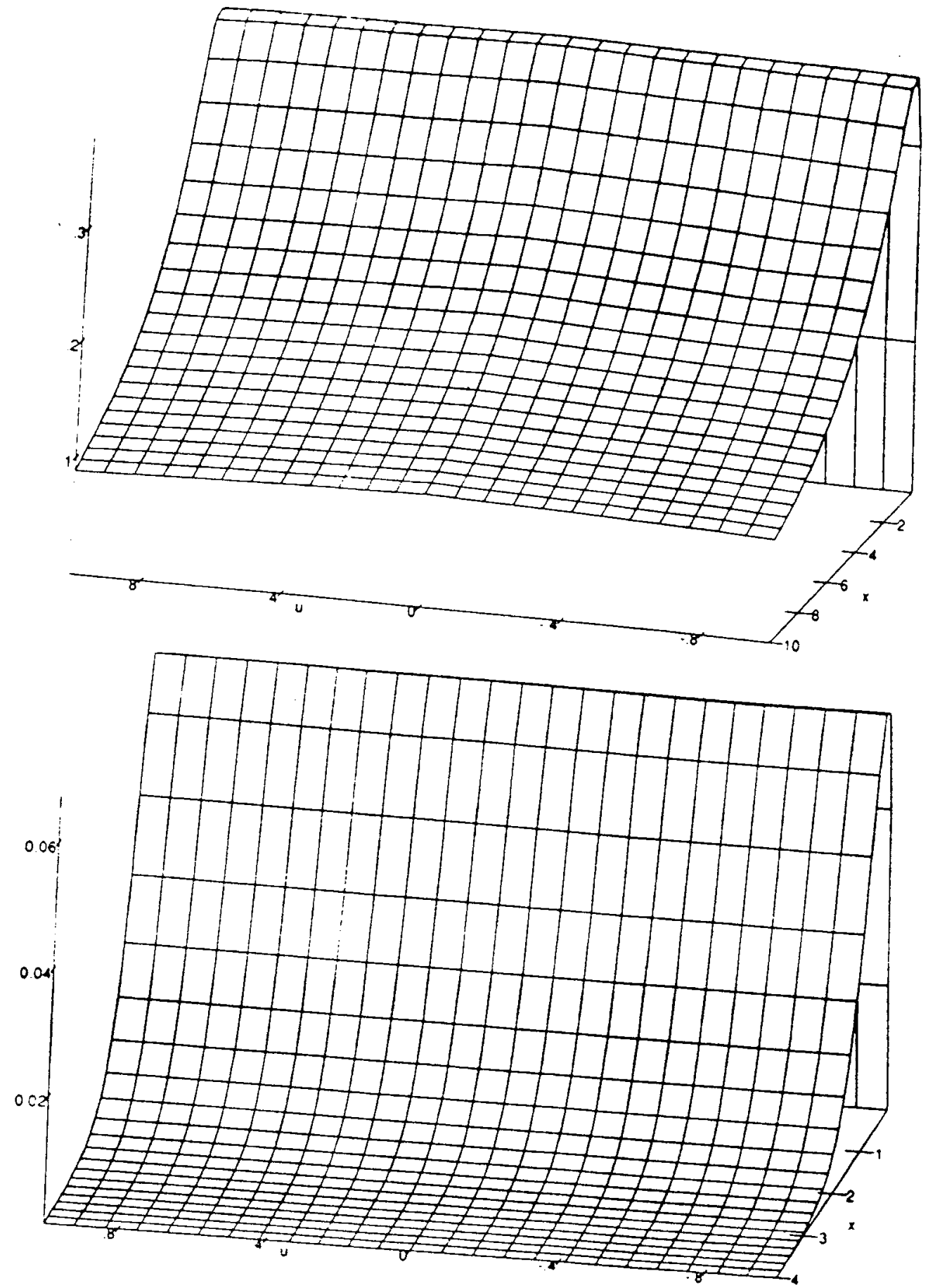

Figure 5.10: Domain integrand, (4.113), $\rho=0.01, j=0$ (top), and $j= \pm 100$. 


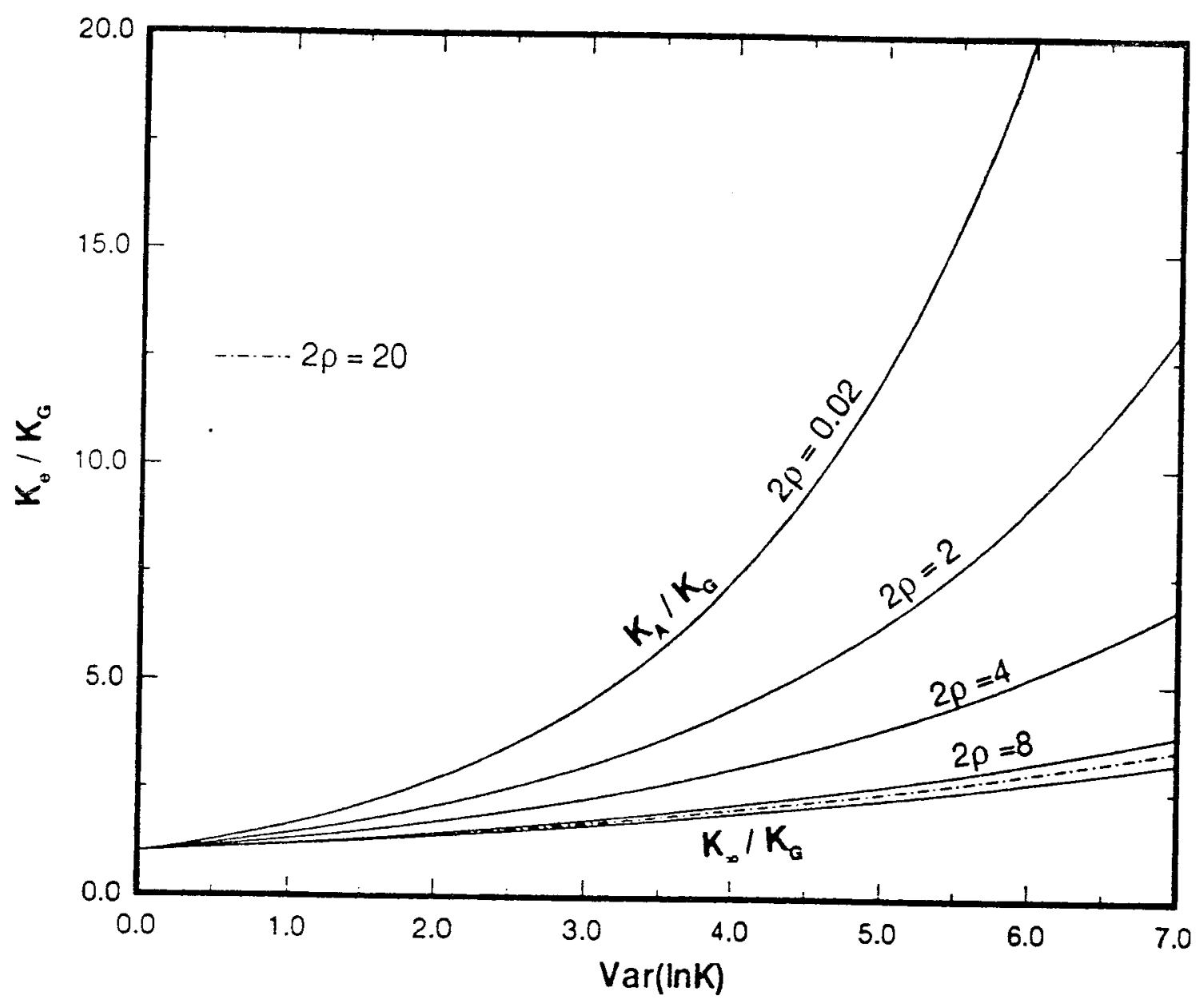

Figure 5.11: Normalized $K_{e}$, equation 4.115 , as a function of $\sigma_{Y}^{2}$ for various $2 \rho$. 


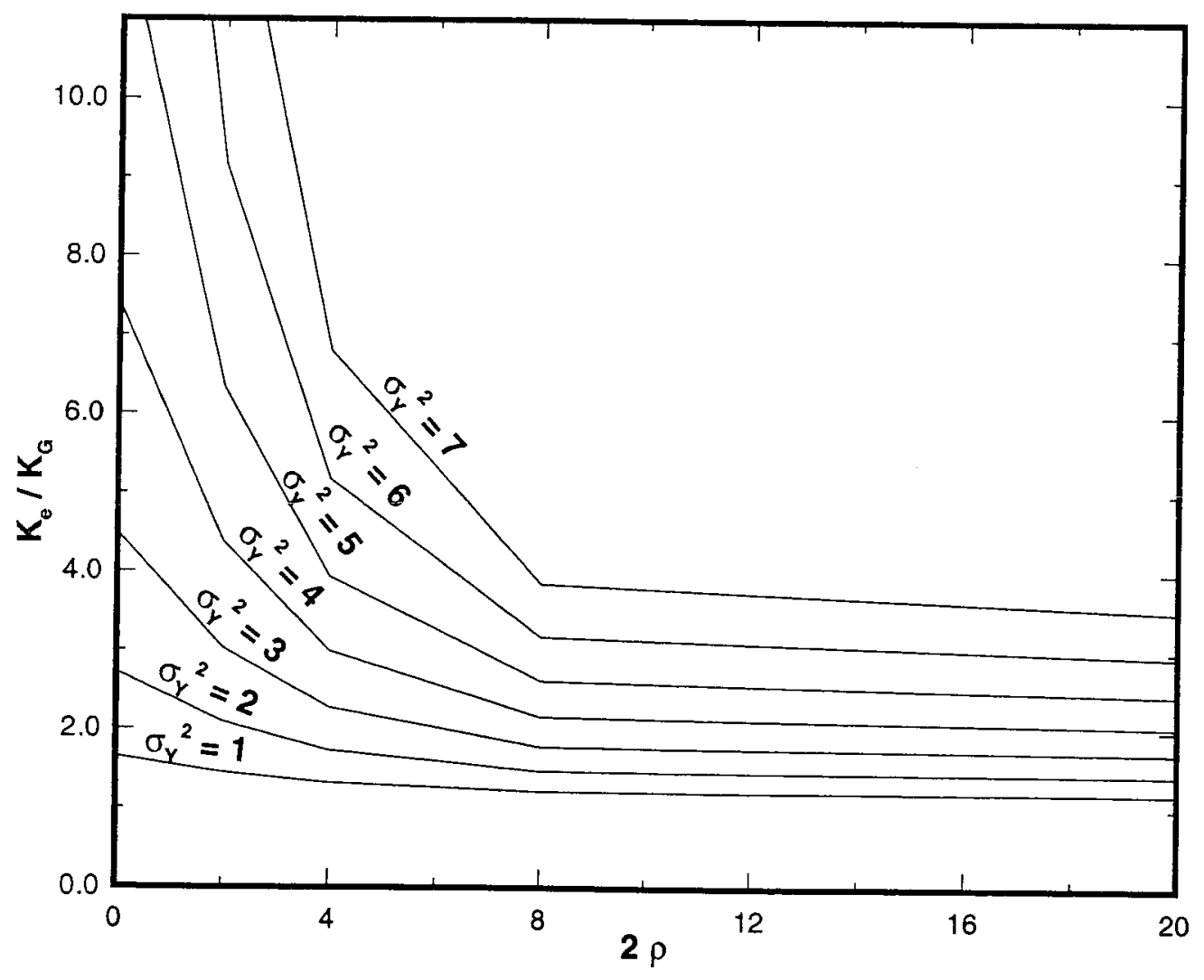

Figure 5.12: Normalized $K_{e}$, equation 4.115 , as a function of $2 \rho$ for various $\partial_{Y}^{2}$. 


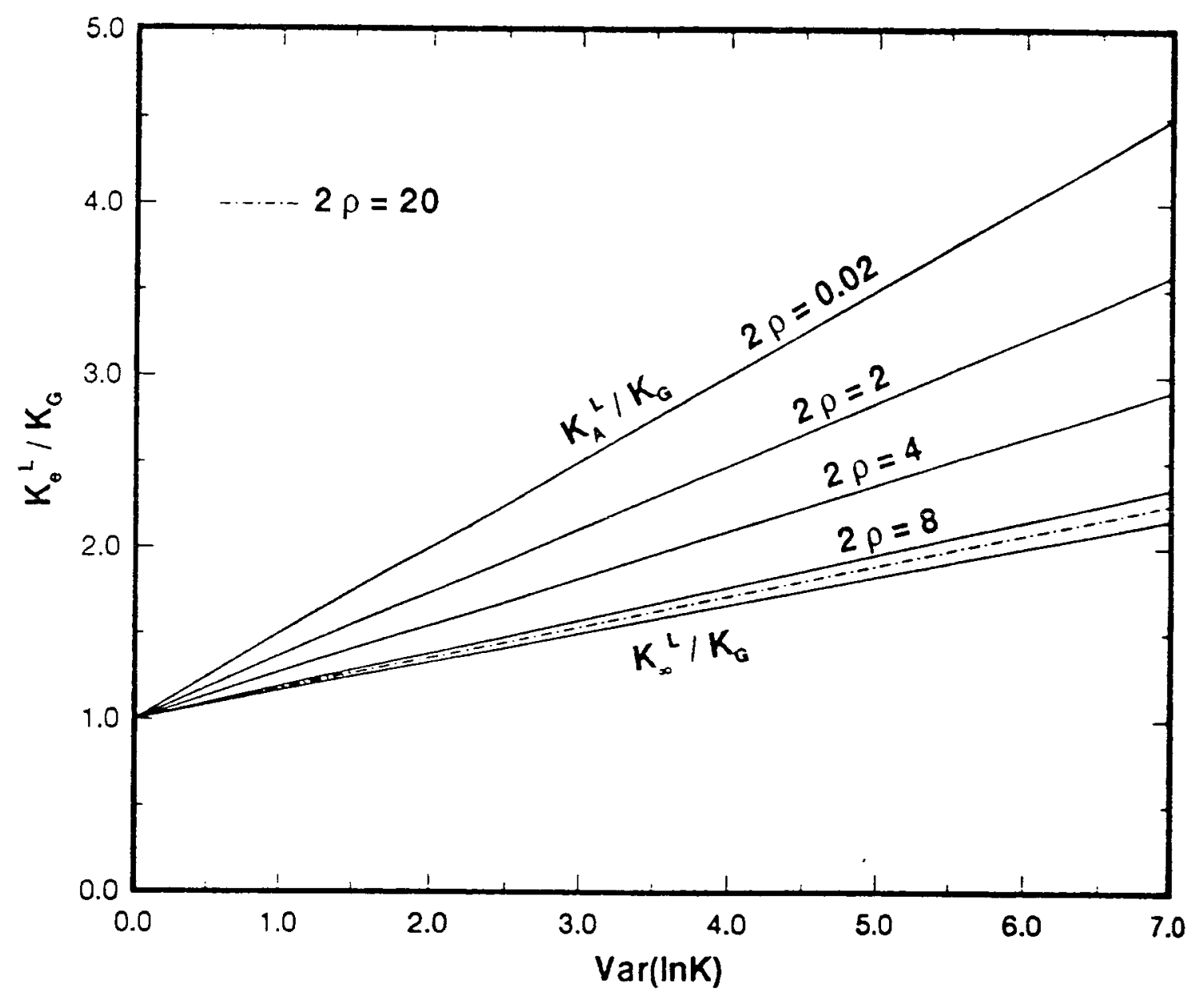

Figure 5.13: Normalized $K_{e}^{L}$, equation 4.114, as a function of $\delta_{Y}^{2}$ and $2 \rho$. 


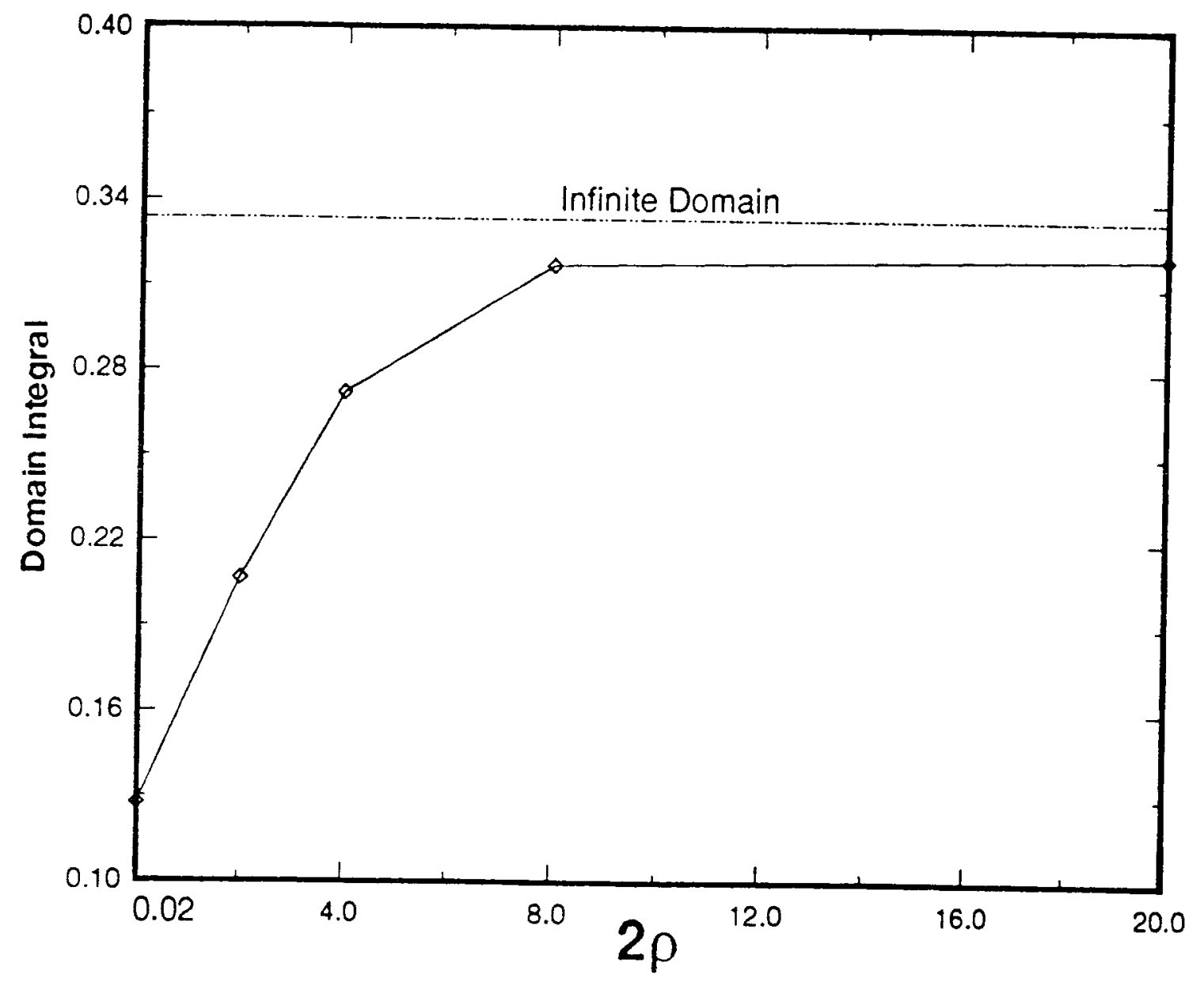

Figure 5.14: Domain integral, equation 4.113, as a function of $2 \rho$. 


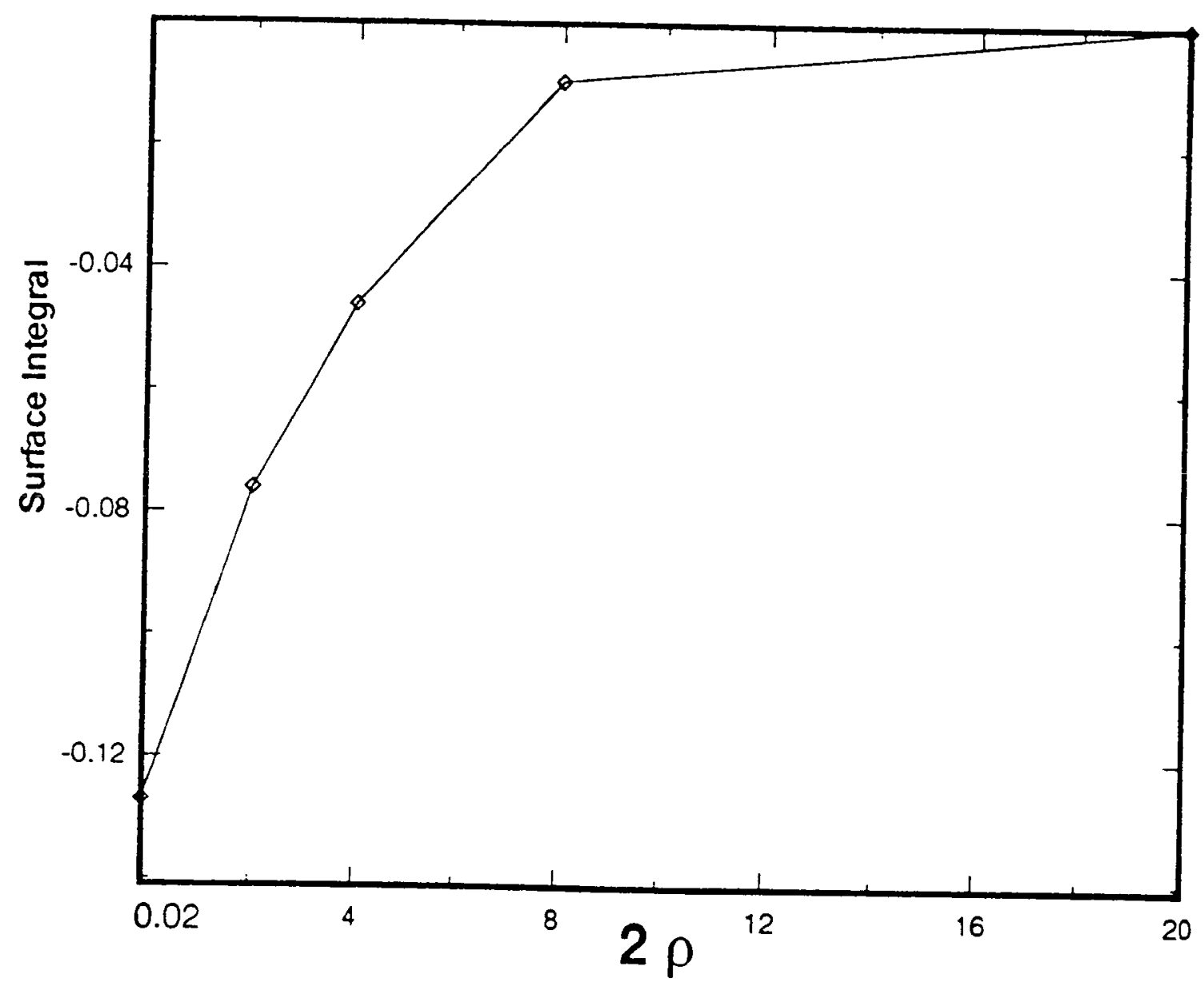

Figure 5.15: Surface integral, equation 4.110, as a function of $2 \rho$. 


\section{CHAPTER 6}

\section{CONCLUSIONS}

Our analysis leads to the following conclusions:

1. The small perturbation method for $\sigma_{Y}^{2} \ll 1$, when coupled with a spectral approach [Bakr et al., 1978; Gutjahr et al., 1978; Gelhar and Axness, 1981, 1983a], cannot address flow in bounded domains. Whereas the spectral approach must assume that head fluctuations are stationary, in bounded domains they are in fact non-stationary [Smith and Freeze, 1979b; Naff and Vecchia, 1986; Rubin and Dagan, 1988, 1989]. When the small perturbation method is coupled with a Green's function approach [Naff and Vecchia, 1986; Rubin and Dagan, 1988, 1989], this limitation disappears.

2. The asymptotic expansion and Green's function approach of Dagan [1989] assumes that $\boldsymbol{\alpha}=\left\langle Y^{\prime}(\boldsymbol{x}) \nabla h^{(1)}(\boldsymbol{x})\right\rangle$ is equal to a constant. This is generally true only if the first order approximation of head, $h^{(1)}$, is stationary, which is not the case near boundaries. Indeed, Dagan [1989, p. 205] provides an example of flow parallel to an impervious boundary where $\boldsymbol{\alpha}$ is not a constant. Furthermore, the above assumption leads him to ignore a first order term in $\sigma_{Y}^{2}$ from the effective hydraulic conductivity. This term, $-K_{G} \nabla h^{(2)}(\boldsymbol{x})$, is recovered by the residual flux theory of Neuman and Orr [1993]. 
3. We have developed expressions for the effective hydraulic conductivity of a three-dimensional, statistically anisotropic or isotropic, medium in the presence of two constant mean head boundaries. Our expressions hold for exponential $Y$ covariance with flow parallel to a principal direction of statistical anisotropy. $K_{e}$ is seen to be a function of $\sigma_{Y}^{2}$ and the ratios $\rho=a / \lambda_{1}, \varepsilon_{2}=\lambda_{2} / \lambda_{1}, \varepsilon_{3}=\lambda_{3} / \lambda_{1}$, where $a$ is half the distance between the boundaries and $\lambda_{i}$ are principal integral scales $(i=1,2,3)$.

4. We have formally shown that as $\rho \rightarrow 0, K_{e}$ reduces to the arithmetic mean, $K_{A}$. This agrees with the numerical result by Desbarats [1992a]. It supports the theoretical finding by Neuman and Orr [1993] that $K_{e}$ reduces to $K_{A}$ when the flow domain $\Omega$ shrinks to the support volume $\omega$. In isotropic media we formally obtain the asymptotic result $K_{e}=K_{G} \exp \left(\sigma_{Y}^{2} / 6\right)$ when $\rho \rightarrow \infty$. The former is the exact solution in infinite domains [Noetinger, 1990; Dykaar and Kitanidis, 1992b; Neuman et al., 1992].

5. When $\varepsilon_{2}=\varepsilon_{3} \rightarrow 0$ or $\varepsilon_{2}=\varepsilon_{3} \rightarrow \infty$ we obtain theoretically the simple expression $K_{e}=K_{G} \exp \left\{\sigma_{Y}^{2}[\exp (-\rho)-0.5]\right\}$. One can think of the former case as mean flow along parallel channels having mutually uncorrelated hydraulic conductivities, and of the latter as mean flow normal to layers having uniform hydraulic conductivities.

6. We have shown that, for small values of $2 \rho$, contrary to Dagan's assertion [1989], the surface integral $\mathcal{S}$ is as important as the domain integral $\mathcal{D}$. In the limit as $\rho \rightarrow 0, \mathcal{S}$ becomes absolutely equal to $\mathcal{D}$ (Sections 5.1 and 5.4). 
7. For statistically isotropic media with arbitrarily large $\sigma_{Y}^{2}$ in three-dimensional flow domains, our analysis indicates that the infinite domain solution becomes a very good approximation to the bounded domain solution when the distance between Dirichlet boundaries exceeds 100 integral scales. For practical purposes, the same happens after 8 integral scales (Figures 5.11 and 5.13). This supports the theoretical finding by Rubin and Dagan [1988], and the numerical results by Desbarats and Dimitrakopoulos [1990], Desbarats [1992a], and Dykaar and Kitanidis [1992b]; it also explains the numerical results by Smith and Freeze [1979b], and Rubin and Gómez-Hernández [1990]. 


\section{APPENDIX A}

\section{DERIVATION OF EXPRESSION FOR THE COVARIANCE OF THE HYDRAULIC CONDUCTIVITY}

The development of expressions from appendix D of Neuman and Orr [1993] is included here for the sake of completeness. Let us commence by performing the transformation $Y(\boldsymbol{x})=\ln K(\boldsymbol{x})$. The random function $Y(\boldsymbol{x})$ is decomposed according to

$$
Y(\boldsymbol{x})=\langle Y(\boldsymbol{x})\rangle+Y^{\prime}(\boldsymbol{x})
$$

with $\langle Y(\boldsymbol{x})\rangle$ the ensemble mean and $Y^{\prime}(\boldsymbol{x})$ a zero mean perturbation. Then

$$
K(\boldsymbol{x})=e^{Y(\boldsymbol{x})}=K_{G}(\boldsymbol{x}) e^{Y^{\prime}(\boldsymbol{x})},
$$

where $K_{G}(\boldsymbol{x})=e^{\langle Y(\boldsymbol{x})\rangle}$ is the geometric mean of $K(\boldsymbol{x})$. If $Y^{\prime}(\boldsymbol{x})$ is Gaussian it can be shown [Mood and Graybill, 1963] that $\left\langle e^{Y^{\prime}(\boldsymbol{x})}\right\rangle=e^{\frac{1}{2} \sigma_{Y}^{2}(\boldsymbol{x})}$ and then

$$
\kappa(\boldsymbol{x})=\langle K(\boldsymbol{x})\rangle=K_{G}(\boldsymbol{x}) e^{\frac{1}{2} \sigma_{Y}^{2}(\boldsymbol{x})},
$$

with

$$
K^{\prime}(\boldsymbol{x})=K(\boldsymbol{x})-\langle K(\boldsymbol{x})\rangle=K_{G}(\boldsymbol{x})\left[e^{Y^{\prime}(\boldsymbol{x})}-e^{\frac{1}{2} \sigma_{Y}^{2}(\boldsymbol{x})}\right]
$$

The autocovariance of $K^{\prime}(\boldsymbol{x})$ is given by

$$
\left\langle K^{\prime}(\boldsymbol{x}) K^{\prime}(\boldsymbol{\chi})\right\rangle=K_{G}(\boldsymbol{x}) K_{G}(\boldsymbol{\chi})\left\langle\left\{e^{Y^{\prime}(\boldsymbol{x})}-e^{\frac{1}{2} \sigma_{Y}^{2}(\boldsymbol{x})}\right\}\right.
$$




$$
\begin{aligned}
& \left.\left\{e^{Y^{\prime}(\boldsymbol{\chi})}-e^{\frac{1}{2} \sigma_{Y}^{2}(\boldsymbol{\chi})}\right\}\right\rangle \\
= & K_{G}(\boldsymbol{x}) K_{G}(\boldsymbol{\chi})\left\{\left\langle e^{Y^{\prime}(\boldsymbol{x})+Y^{\prime}(\boldsymbol{\chi})}\right\rangle\right. \\
& \left.-e^{\frac{1}{2}\left(\sigma_{Y}^{2}(\boldsymbol{x})+\sigma_{Y}^{2}(\boldsymbol{\chi})\right)}\right\}
\end{aligned}
$$

where some intermediate steps have been omitted. For normally distributed $Y^{\prime}(\boldsymbol{x})$ we have [Mood and Graybill, 1963] exactly

$$
\left\langle e^{Y^{\prime}(\boldsymbol{x})+Y^{\prime}(\boldsymbol{\chi})}\right\rangle=\exp \left[\frac{1}{2}\left(\sigma_{Y}^{2}(\boldsymbol{x})+\sigma_{Y}^{2}(\boldsymbol{\chi})+2\left\langle Y^{\prime}(\boldsymbol{x}) Y^{\prime}(\boldsymbol{\chi})\right\rangle\right)\right]
$$

Substituting (A.6) in (A.5) we obtain

$$
\begin{aligned}
\left\langle K^{\prime}(\boldsymbol{x}) K^{\prime}(\boldsymbol{\chi})\right\rangle= & K_{G}(\boldsymbol{x}) K_{G}(\boldsymbol{\chi}) e^{\frac{1}{2}\left(\sigma_{Y}^{2}(\boldsymbol{x})+\sigma_{Y}^{2}(\boldsymbol{\chi})\right)} \\
& {\left[e^{\left\langle Y^{\prime}(\boldsymbol{x}) Y^{\prime}(\boldsymbol{\chi})\right\rangle}-1\right] }
\end{aligned}
$$

For the statistically homogeneous case (A.3) and (A.7) become

$$
\begin{gathered}
\kappa=K_{G} e^{\frac{1}{2} \sigma_{Y}^{2}} \\
\left\langle K^{\prime}(\boldsymbol{x}) K^{\prime}(\boldsymbol{\chi})\right\rangle=K_{G}^{2} e^{\sigma_{Y}^{2}}\left[e^{\langle Y(\boldsymbol{x}) Y(\boldsymbol{\chi})\rangle}-1\right]
\end{gathered}
$$

respectively. 


\section{APPENDIX B}

\section{DERIVATION OF PARSEVAL'S IDENTITIES}

Let us define the Fourier Transform (FT) pair

$$
\begin{gathered}
f(\boldsymbol{\chi})=\frac{1}{(2 \pi)^{m / 2}} \int_{\boldsymbol{k}} F(\boldsymbol{k}) e^{-i \boldsymbol{k} \cdot \boldsymbol{\chi}} d \boldsymbol{k} \\
F^{(m)}(\boldsymbol{k})=\frac{1}{(2 \pi)^{m / 2}} \int_{\boldsymbol{\chi}} f(\boldsymbol{\chi}) e^{i \boldsymbol{k} \cdot \boldsymbol{\chi}} d \boldsymbol{\chi}
\end{gathered}
$$

where $m=1,2,3$ is the dimension of the space domain and $\boldsymbol{k}$ is the wave number vector in the corresponding Fourier space. We want to evaluate the integral

$$
H=\int_{x_{2}=-\infty}^{+\infty} \int_{x_{3}=-\infty}^{+\infty} f\left(x_{1}, x_{2}, x_{3}\right) g\left(x_{1}, x_{2}, x_{3}\right) d x_{2} d x_{3} .
$$

Let us define now the two-dimensional FT

$$
\begin{gathered}
G^{(2)}\left(-k_{2},-k_{3} ; \chi_{1}\right)=\frac{1}{2 \pi} \int_{\chi_{2}=-\infty}^{+\infty} \int_{\chi_{3}=-\infty}^{+\infty} e^{-i\left(k_{2} \chi_{2}+k_{3} \chi_{3}\right)} g\left(\chi_{1}, \chi_{2}, \chi_{3}\right) d \chi_{2} d \chi_{3} \\
F^{(2)}\left(k_{2}, k_{3} ; \chi_{1}\right)=\frac{1}{2 \pi} \int_{\chi_{2}=-\infty}^{+\infty} \int_{\chi_{3}=-\infty}^{+\infty} e^{i\left(k_{2} \chi_{2}+k_{3} \chi_{3}\right)} f\left(\chi_{1}, \chi_{2}, \chi_{3}\right) d \chi_{2} d \chi_{3}
\end{gathered}
$$

Now,

$$
\begin{aligned}
& \int_{-\infty}^{+\infty} \int_{-\infty}^{+\infty} F^{(2)}\left(k_{2}, k_{3} ; x_{1}\right) G^{(2)}\left(-k_{2},-k_{3} ; x_{1}\right) e^{-i\left(k_{2} \chi_{2}+k_{3} x_{3}\right)} d k_{2} d k_{3} \\
= & \int_{k_{2}=-\infty}^{+\infty} \int_{k_{3}=-\infty}^{+\infty} F^{(2)}\left(k_{2}, k_{3} ; x_{1}\right) e^{-i\left(k_{2} \chi_{2}+k_{3} \chi_{3}\right)}\left\{\frac{1}{2 \pi}\right. \\
& \left.\int_{x_{2}=-\infty}^{+\infty} \int_{x_{3}=-\infty}^{+\infty} e^{-i\left(k_{2} x_{2}+k_{3} x_{3}\right)} g\left(x_{1}, x_{2}, x_{3}\right) d x_{2} d x_{3}\right\} d k_{2} d k_{3} \\
= & \int_{x_{2}=-\infty}^{+\infty} \int_{x_{3}=-\infty}^{+\infty} g\left(x_{1}, x_{2}, x_{3}\right)\left\{\frac{1}{2 \pi} \int_{k_{2}=-\infty}^{+\infty} \int_{k_{3}=-\infty}^{+\infty}\right. \\
& \left.e^{-i k_{2}\left(\chi_{2}+x_{2}\right)} e^{-i k_{3}\left(\chi_{3}+x_{3}\right)} F^{(2)}\left(k_{2}, k_{3} ; x_{1}\right) d k_{2} d k_{3}\right\} d x_{2} d x_{3} \\
= & \int_{x_{2}=-\infty}^{+\infty} \int_{x_{3}=-\infty}^{+\infty} g\left(x_{1}, x_{2}, x_{3}\right) f\left(x_{1}, x_{2}+\chi_{2}, x_{3}+\chi_{3}\right) d x_{2} d x_{3} .
\end{aligned}
$$


Setting $\chi_{2}=\chi_{3}=0$, we obtain Parseval's identity

$$
\begin{aligned}
& \int_{k_{2}=-\infty}^{+\infty} \int_{k_{3}=-\infty}^{+\infty} F^{(2)}\left(k_{2}, k_{3} ; x_{1}\right) G^{(2)}\left(-k_{2},-k_{3} ; x_{1}\right) d k_{2} d k_{3} \\
= & \int_{x_{2}=-\infty}^{+\infty} \int_{x_{3}=-\infty}^{+\infty} g\left(x_{1}, x_{2}, x_{3}\right) f\left(x_{1}, x_{2}, x_{3}\right) d x_{2} d x_{3} .
\end{aligned}
$$

Now,

$$
\begin{aligned}
& \int_{\boldsymbol{k}} F^{(3)}\left(k_{1}, k_{2}, k_{3}\right) G^{(3)}\left(-k_{1},-k_{2},-k_{3}\right) e^{-i \boldsymbol{k} \cdot \boldsymbol{\chi}} d k_{1} d k_{2} d k_{3} \\
= & \int_{\boldsymbol{k}} F^{(3)}\left(k_{1}, k_{2}, k_{3}\right) e^{-i \boldsymbol{k} \cdot \boldsymbol{\chi}}\left\{\frac{1}{(2 \pi)^{3 / 2}} \int_{\boldsymbol{x}} g\left(x_{1}, x_{2}, x_{3}\right) e^{-i \boldsymbol{k} \cdot \boldsymbol{x}} d \boldsymbol{x}\right\} d \boldsymbol{k} \\
= & \int_{\boldsymbol{x}} g\left(x_{1}, x_{2}, x_{3}\right)\left\{\frac{1}{(2 \pi)^{3 / 2}} \int_{\boldsymbol{k}} F^{(3)}\left(k_{1}, k_{2}, k_{3}\right) e^{-i \boldsymbol{k} \cdot(\boldsymbol{x}+\boldsymbol{\chi})} d \boldsymbol{k}\right\} d \boldsymbol{x} \\
= & \int_{\boldsymbol{x}} g\left(x_{1}, x_{2}, x_{3}\right) f\left(x_{1}+\chi_{1}, x_{2}+\chi_{2}, x_{3}+\chi_{3}\right) d x_{1} d x_{2} d x_{3} .
\end{aligned}
$$

Setting $\chi_{1}=\chi_{2}=\chi_{3}=0$, we have for the three-dimensional case $(m=3)$

$$
\int_{\boldsymbol{k}} F^{(m)}(\boldsymbol{k}) G^{(m)}(-\boldsymbol{k}) d \boldsymbol{k}=\int_{\boldsymbol{x}} g(\boldsymbol{x}) f(\boldsymbol{x}) d \boldsymbol{x} .
$$

Parseval's identity in the form of (B.9) can be easily shown to hold in general for $m=1,2,3$. Equation B.9 is related to (B.7) as follows

$$
\begin{aligned}
& \int_{\boldsymbol{x}} g\left(x_{1}, x_{2}, x_{3}\right) f\left(\chi_{1}+x_{1}, \chi_{2}+x_{2}, \chi_{3}+x_{3}\right) d x_{1} d x_{2} d x_{3} \\
= & \int_{\boldsymbol{k}} F^{(3)}\left(k_{1}, k_{2}, k_{3}\right) G^{(3)}\left(-k_{1},-k_{2},-k_{3}\right) e^{-i \boldsymbol{k} \cdot \boldsymbol{\chi}} d \boldsymbol{k} \\
= & \int_{\boldsymbol{k}} F^{(3)}\left(k_{1}, k_{2}, k_{3}\right) e^{-i \boldsymbol{k} \cdot \boldsymbol{\chi}}\left\{\frac{1}{(2 \pi)^{3 / 2}} \int_{\boldsymbol{x}} g\left(x_{1}, x_{2}, x_{3}\right) e^{-i \boldsymbol{k} \cdot \boldsymbol{x}} d \boldsymbol{x}\right\} d \boldsymbol{k} \\
= & \int_{\boldsymbol{k}} F^{(3)}\left(k_{1}, k_{2}, k_{3}\right) e^{-i \boldsymbol{k} \cdot \boldsymbol{\chi}}\left\{\frac{1}{\sqrt{2 \pi}} \int_{x_{1}=-\infty}^{+\infty} e^{-i k_{1} x_{1}} G^{(2)}\left(-k_{2},-k_{3} ; x_{1}\right) d x_{1}\right\} d \boldsymbol{k} \\
= & \int_{x_{1}=-\infty}^{+\infty} G^{(2)}\left(-k_{2},-k_{3} ; x_{1}\right) \int_{k_{2}} \int_{k_{3}}\left\{\frac{1}{\sqrt{2 \pi}} \int_{k_{1}} F^{(3)}\left(k_{1}, k_{2}, k_{3}\right) e^{-i k_{1}\left(\chi_{1}+x_{1}\right)} d k_{1}\right\} \\
& e^{-i\left(k_{2} \chi_{2}+k_{3} \chi_{3}\right)} d k_{2} d k_{3} d x_{1} \\
= & \int_{x_{1}=-\infty}^{+\infty}\left\{\int_{k_{2}} \int_{k_{3}} G^{(2)}\left(-k_{2},-k_{3} ; x_{1}\right) F^{(2)}\left(k_{2}, k_{3} ; \chi_{1}+x_{1}\right)\right. \\
& \left.e^{-i\left(k_{2} \chi_{2}+k_{3} \chi_{3}\right)} d k_{2} d k_{3}\right\} d x_{1} .
\end{aligned}
$$


Setting again $\chi=0$,

$$
\begin{aligned}
& \int_{\boldsymbol{x}} g(\boldsymbol{x}) f(\boldsymbol{x}) d \boldsymbol{x}=\int_{x_{1}=-\infty}^{+\infty} \\
& \left\{\int_{k_{2}} \int_{k_{3}} G^{(2)}\left(-k_{2},-k_{3} ; x_{1}\right) F^{(2)}\left(k_{2}, k_{3} ; x_{1}\right) d k_{2} d k_{3}\right\} d x_{1} .
\end{aligned}
$$




\section{APPENDIX C}

\section{FOURIER TRANSFORM OF MIXED DERIVATIVES OF 3- AND 2-DIMENSIONAL INFINITE GREEN'S FUNCTION}

The 3-dimensional Fourier Transform (FT) of the infinite Green's function, ${\hat{G_{F}}}^{\left({ }^{(3)}\right.}$, is given by

$$
{\hat{G_{F}}}^{(3)}=\frac{1}{(2 \pi)^{3 / 2}} \int_{-\infty}^{+\infty} \int_{-\infty}^{+\infty} \int_{-\infty}^{+\infty} \frac{1}{4 \pi \eta} e^{i \boldsymbol{k} \cdot \boldsymbol{\chi}} d \chi_{1} d \chi_{2} d \chi_{3}
$$

where

$$
\begin{gathered}
\boldsymbol{\chi}=\left(\chi_{1}, \chi_{2}, \chi_{3}\right)^{T} \quad \boldsymbol{x}=\left(x_{1}, x_{2}, x_{3}\right)^{T}, \\
\boldsymbol{\eta}=\boldsymbol{\chi}-\boldsymbol{x}
\end{gathered}
$$

$\boldsymbol{k}$ is the 3 -dimensional wave number vector, and norms of vectors are again represented by unaccentuated versions of their boldface symbols. Then we can write

$$
{\hat{G_{F}}}^{(3)}=\frac{1}{(2 \pi)^{3 / 2}} e^{i \boldsymbol{k} \cdot \boldsymbol{x}} \int_{-\infty}^{+\infty} \int_{-\infty}^{+\infty} \int_{-\infty}^{+\infty} \frac{1}{4 \pi \eta} e^{i \boldsymbol{k} \cdot \boldsymbol{\eta}} d \eta_{1} d \eta_{2} d \eta_{3}
$$

which in spherical coordinates becomes

$$
\begin{aligned}
{\hat{G_{F}}}^{(3)} & =\frac{1}{4 \pi} \frac{1}{(2 \pi)^{3 / 2}} e^{i \boldsymbol{k} \cdot \boldsymbol{x}} \int_{\eta=0}^{+\infty} \int_{\theta=0}^{\pi} \int_{\psi=0}^{2 \pi} \\
& \frac{1}{\eta} e^{i k \eta \cos \theta} \eta^{2} \sin \theta d \theta d \eta d \psi \\
& \frac{1}{2} \frac{1}{(2 \pi)^{3 / 2}} e^{i \boldsymbol{k} \cdot \boldsymbol{x}} \int_{\eta=0}^{+\infty} \eta\left\{\int_{\theta=0}^{\pi} e^{i k \eta \cos \theta} \sin \theta d \theta\right\} d \eta \\
& =\frac{1}{(2 \pi)^{3 / 2}} e^{i \boldsymbol{k} \cdot \boldsymbol{x}} \int_{\eta=0}^{+\infty} \frac{\sin (k \eta)}{k} d \eta \\
& =\frac{1}{(2 \pi)^{3 / 2}} e^{i \boldsymbol{k} \cdot \boldsymbol{x}} \frac{1}{k^{2}},
\end{aligned}
$$


where the integrals over $\theta$ and $\eta$ were evaluated using Gradshteyn and Ryzhik [1980, p. 482[5] and 966[8.464.1]] and Haberman [1987, p. 365].

The FT of the 3-(or 2-) dimensional infinite Green's function $G_{F}$ can also be calculated by applying the FT directly on their governing equation 3.34 ,

$$
\begin{aligned}
F T^{(m)}\left[\nabla^{2} G_{F}\right] & =-F T^{(m)}[\delta(\boldsymbol{\chi}-\boldsymbol{x})] \\
& =-\frac{1}{(2 \pi)^{m / 2}} e^{i \boldsymbol{k} \cdot \boldsymbol{x}}
\end{aligned}
$$

and utilizing the relationship

$$
F T^{(m)}\left[\frac{\partial^{2}}{\partial \chi_{i}^{2}} G_{F}\right]=-k_{i}^{2} \hat{G}_{F}^{(m)} \quad i=1, \cdots, m
$$

we obtain

$$
{\hat{G_{F}}}^{(m)}=\frac{1}{(2 \pi)^{m / 2}} e^{i \boldsymbol{k} \cdot \boldsymbol{x}} \frac{1}{k^{2}}
$$

where $m$ is the Euclidean (and equivalent Fourier) space dimension and all vectors (and their norms) should be considered in the corresponding space. Based on the relationships,

$$
\begin{gathered}
\frac{\partial}{\partial x_{1}} G_{F}=-\frac{\partial}{\partial \chi_{1}} G_{F} \\
F T^{(m)}\left[\frac{\partial}{\partial \chi_{i}} G_{F}\right]=-i k_{i} \hat{G}_{F}{ }^{(m)} \quad i=1, \cdots, m
\end{gathered}
$$

we finally have

$$
\Psi(\boldsymbol{k})=F T^{(m)}\left[\nabla_{x} \nabla_{\chi}^{T} G_{F}(\boldsymbol{\eta})\right]=\frac{1}{(2 \pi)^{\frac{m}{2}}} \frac{\boldsymbol{k} \boldsymbol{k}^{T}}{k^{2}} e^{i \boldsymbol{k} \cdot \boldsymbol{x}}
$$




\section{APPENDIX D \\ EVALUATION OF THE LEGENDRE POLYNOMIAL $P_{1}(x)$}

The Legendre Polynomial of order one [Erdelyi et al., 1954, p. 424] is given by

$$
P_{1}(x)=\frac{1}{2} \frac{d}{d x}\left(x^{2}-1\right)
$$

where $x$, equation $\mathrm{J} .11$, is given by

$$
x=\frac{1}{\left(1+k^{*^{2}}\right)^{1 / 2}}=x\left(k^{*}\right) .
$$

Here $k^{*}$ is the norm of the vector $\boldsymbol{k}^{*}=\boldsymbol{L} \boldsymbol{k}$, where $\boldsymbol{L}$ is a $2 \times 2$ diagonal matrix with elements $\lambda_{1}$ and $\lambda_{2}$, and $\boldsymbol{k}=\left(k_{1}, k_{2}\right)^{T}$ is a two-dimensional wave number vector. From (D.1) and (D.2) we have that

$$
g\left(k^{*}\right)=x^{2}-1=-\frac{k^{*^{2}}}{1+k^{*^{2}}} .
$$

The derivative of $g\left(k^{*}\right)$ with respect to $x$ is expressed as

$$
\frac{d}{d x} g\left(k^{*}\right)=\frac{d g}{d k^{*}} \frac{d k^{*}}{d x}=\frac{d g}{d k^{*}} /\left(\frac{d x}{d k^{*}}\right) .
$$

The derivatives of $g$ and $x$ with respect to $k^{*}$ are evaluated as

$$
\frac{d g}{d k^{*}}=-\frac{2 k^{*}}{\left(1+k^{*^{2}}\right)^{2}}
$$

and

$$
\frac{d x}{d k^{*}}=-\frac{k^{*}}{\left(1+k^{*^{2}}\right)^{3 / 2}} .
$$


Upon substitution of (D.5) and (D.6) in (D.4) the Legendre Polynomial of order one becomes

$$
P_{1}\left(k^{*}\right)=\frac{1}{\left(1+k^{*^{2}}\right)^{1 / 2}}
$$




\section{APPENDIX E}

\section{EVALUATION OF THE INTEGRAL $\mathcal{E}$}

Sanchez-Villa and Carrera [1993] evaluated integral $\mathcal{E}$, given in (J.13), as

$$
\mathcal{E}=\frac{\lambda_{1} \lambda_{2} \sigma_{Y}^{2}}{2 \pi} \int_{\boldsymbol{k}} \frac{\boldsymbol{k} \boldsymbol{k}^{T}}{k^{2}} \frac{d \boldsymbol{k}}{\left[1+\left(\lambda_{1} k_{1}\right)^{2}+\left(\lambda_{2} k_{2}\right)^{2}\right]^{3 / 2}}
$$

by expressing $\boldsymbol{k}^{*}=\left(\lambda_{1} k_{1}, \lambda_{2} k_{2}\right)^{T}$ in polar coordinates $\boldsymbol{k}^{*}=k^{*}(\cos \phi, \sin \phi)^{T}$, thus transforming $\mathcal{E}_{i j}$ into

$$
\mathcal{E}_{i j}=\frac{\sigma_{Y}^{2}}{2 \pi} \int_{k^{*}=0}^{\infty} \int_{\phi=0}^{2 \pi} \frac{m_{i} m_{j} / \lambda_{i} \lambda_{j}}{\frac{\cos ^{2} \phi}{\lambda_{1}^{2}}+\frac{\sin ^{2} \phi}{\lambda_{2}^{2}}} \frac{k^{*}}{\left(1+k^{*^{2}}\right)^{3 / 2}} d k^{*} d \phi
$$

where $i, j=1,2, m_{1}=\cos \phi$ and $m_{2}=\sin \phi$. The integral with respect to $k^{*}$ equals one [Gradshteyn and Ryzhik, 1980, p. 83[6]], and thus the components of $\mathcal{E}_{i j}$ can be evaluated by performing the integration over $\phi$. Defining $e=\lambda_{2} / \lambda_{1}$ we obtain

$$
\begin{aligned}
\mathcal{E}_{11} & =\frac{\sigma_{Y}^{2}}{2 \pi} \int_{\phi=0}^{2 \pi} \frac{\cos ^{2} \phi}{\cos ^{2} \phi+\frac{\sin ^{2} \phi}{e^{2}}} d \phi=\frac{2 \sigma_{Y}^{2}}{\pi} \int_{t=0}^{\infty} \frac{1}{1+\frac{t^{2}}{e^{2}}} \frac{d t}{1+t^{2}} \\
& =\frac{2 \sigma_{Y}^{2}}{\pi} \frac{1}{1-e^{2}}\left[\int_{t=0}^{\infty} \frac{d t}{1+\frac{t^{2}}{e^{2}}}-e^{2} \int_{t=0}^{\infty} \frac{d t}{1+t^{2}}\right]=\frac{\lambda_{2} \sigma_{Y}^{2}}{\lambda_{1}+\lambda_{2}}
\end{aligned}
$$

where the transformation $t=t g \phi$ was employed and some intermediate steps were omitted. Similarly for the integral $\mathcal{E}_{22}$ we have

$$
\begin{aligned}
\mathcal{E}_{22} & =\frac{\sigma_{Y}^{2}}{2 \pi} \int_{\phi=0}^{2 \pi} \frac{\sin ^{2} \phi}{e^{2} \cos ^{2} \phi+\sin ^{2} \phi} d \phi=\frac{2 \sigma_{Y}^{2}}{\pi} \int_{t=0}^{\infty} \frac{1}{1+e^{2} t^{2}} \frac{d t}{1+t^{2}} \\
& =\frac{2 \sigma_{Y}^{2}}{\pi} \frac{1}{1-e^{2}}\left[\int_{t=0}^{\infty} \frac{-e^{2} d t}{1+e^{2} t^{2}}+\int_{t=0}^{\infty} \frac{d t}{1+t^{2}}\right]=\frac{\lambda_{1} \sigma_{Y}^{2}}{\lambda_{1}+\lambda_{2}}
\end{aligned}
$$


where in this case the transformation $t=\operatorname{cotg} \phi$ was used. Integrals $\mathcal{E}_{12}$ and $\mathcal{E}_{21}$ are immediately seen to equal zero because of the integration of an odd function over a symmetric interval. 


\section{APPENDIX F}

\section{DERIVATION OF EQUATION 3.35}

The three-dimensional $\boldsymbol{K}_{e}$, equation 3.33 , is given by

$$
\boldsymbol{K}_{e}=K_{G} e^{\sigma_{Y}^{2} / 2}\left\{\boldsymbol{I}-\int_{\Omega_{\infty}}\left[e^{C_{Y}(\boldsymbol{\chi}-\boldsymbol{x})}-1\right] \nabla_{x} \nabla_{\chi}^{T} G_{F}(\boldsymbol{\chi}, \boldsymbol{x}) d \boldsymbol{\chi}\right\} .
$$

Let us define the three-dimensional Fourier Transforms (FT),

$$
\begin{gathered}
\Phi^{(3)}(-\boldsymbol{k})=\frac{1}{(2 \pi)^{\frac{3}{2}}} \int_{-\infty}^{+\infty} \int_{-\infty}^{+\infty} \int_{-\infty}^{+\infty}\left[e^{C_{Y}(\boldsymbol{\eta})}-1\right] e^{-i \boldsymbol{k} \cdot \boldsymbol{\chi}} d \boldsymbol{\chi} \\
\Psi^{(3)}(\boldsymbol{k})=\frac{1}{(2 \pi)^{\frac{3}{2}}} \int_{-\infty}^{+\infty} \int_{-\infty}^{+\infty} \int_{-\infty}^{+\infty} \nabla_{x} \nabla_{\chi}^{T} G_{F}(\boldsymbol{x}, \boldsymbol{\chi}) e^{i \boldsymbol{k} \cdot \boldsymbol{\chi}} d \boldsymbol{\chi}
\end{gathered}
$$

where $\boldsymbol{k}=\left(k_{1}, k_{2}, k_{3}\right)^{T}$ is a three-dimensional wave number vector and $\boldsymbol{\eta}=\boldsymbol{\chi}-\boldsymbol{x}$. Then, using Parseval's identity (Appendix B), we can write (F.1) as

$$
\boldsymbol{K}_{e}=K_{G} e^{\frac{1}{2} \sigma_{Y}^{2}}\left\{\boldsymbol{I}-\int_{\boldsymbol{k}} \Phi^{(3)}(-\boldsymbol{k}) \Psi^{(3)}(\boldsymbol{k}) d \boldsymbol{k}\right\} .
$$

From Appendix C we have that

$$
\Psi^{(3)}(\boldsymbol{k})=\Psi_{I}(\boldsymbol{k}) e^{i \boldsymbol{k} \cdot \boldsymbol{x}} \quad ; \quad \Psi_{I}(\boldsymbol{k})=\frac{1}{(2 \pi)^{\frac{3}{2}}} \frac{\boldsymbol{k} \boldsymbol{k}^{T}}{k^{2}} .
$$

Expanding the term $e^{C_{Y}(\boldsymbol{\eta})}-1$ in Taylor series we obtain

$$
\Phi^{(3)}(-\boldsymbol{k})=\frac{1}{(2 \pi)^{\frac{3}{2}}} \sum_{m=1}^{\infty} \frac{1}{m !} \int_{\chi} C_{Y}^{m}(\boldsymbol{\eta}) e^{-i \boldsymbol{k} \cdot \boldsymbol{\chi}} d \boldsymbol{\chi}
$$


The FT of the m-power of the covariance function is given (Appendix G) as

$$
F T_{(m)}^{(3)}(-\boldsymbol{k})=\sqrt{\frac{8}{\pi}} \operatorname{det}(\boldsymbol{L}) \sigma_{Y}^{2 m} \frac{m e^{-i \boldsymbol{k} \cdot \boldsymbol{x}}}{\left[m^{2}+\left(\lambda_{1} k_{1}\right)^{2}+\left(\lambda_{2} k_{2}\right)^{2}+\left(\lambda_{3} k_{3}\right)^{2}\right]^{2}}
$$

where $m=1,2, \ldots$ Equation F.6 using (F.7) becomes

$$
\Phi^{(3)}(-\boldsymbol{k})=\Phi_{I}(-\boldsymbol{k}) e^{-i \boldsymbol{k} \cdot \boldsymbol{x}}
$$

with

$$
\Phi_{I}(-\boldsymbol{k})=\sqrt{\frac{8}{\pi}} \operatorname{det}(\boldsymbol{L}) \sum_{m=1}^{\infty} \frac{\sigma_{Y}^{2 m}}{(m-1) !} \frac{1}{\left[m^{2}+\left(\lambda_{i} k_{i}\right)^{2}\right]^{2}}
$$

where summation over the index $i=1,2,3$ is implied. From (F.8), (F.9) and (F.5) we observe that $\Phi^{(3)}$ is an even function, whereas $\Psi^{(3)}$ is an odd function in $k_{i}(i=1,2,3)$. Thus, for the off-diagonal terms of $\boldsymbol{K}_{e}$ the integral in (F.4) becomes zero (due to the integration of an odd function over a symmetric interval) and consequently $\boldsymbol{K}_{e_{i j}}=0$, $i, j=1,2,3$ and $i \neq j$. The expression for the principal components of the effective hydraulic conductivity using (F.5), (F.8) and (F.9) then becomes

$$
K_{e_{i i}}=K_{G} e^{\frac{1}{2} \sigma_{Y}^{2}}\left\{1-\frac{1}{(2 \pi)^{\frac{3}{2}}} \int_{\boldsymbol{k}} \frac{\boldsymbol{k}^{T}}{k^{2}} \Phi_{I}(-\boldsymbol{k}) d \boldsymbol{k}\right\} .
$$

Let us define now a coordinate system $\tilde{\boldsymbol{\chi}}$ that is aligned in the direction of the mean flow $\langle\boldsymbol{q}\rangle$ (with components $\left\langle q_{1}\right\rangle \neq 0,\left\langle q_{2}\right\rangle=\left\langle q_{3}\right\rangle=0$ ), and $\tilde{\boldsymbol{k}}$ is a wave-number vector in this system. Then, coordinates $\chi$ are related to $\tilde{\chi}$ through $\chi=\boldsymbol{\beta} \tilde{\chi}$, where $\boldsymbol{\beta}$ is the matrix of direction cosines $\beta_{i j}$ between $\chi_{i}$ and $\tilde{\chi}_{j}, i, j=1,2,3$ [Gelhar and Axness, 1981; Neuman et al., 1987]. The same relationship, $\boldsymbol{k}=\boldsymbol{\beta} \tilde{\boldsymbol{k}}$, holds for the wave-number vectors. Let us also designate with $\lambda_{\mu}$ the length of a radius vector parallel to $\langle\boldsymbol{q}\rangle$ corresponding to an ellipsoid having semi-axes $\lambda_{1}, \lambda_{2}$ and $\lambda_{3}$ oriented along $\chi_{1}, \chi_{2}$ and $\chi_{3}$, respectively. The latter is given by

$$
\lambda_{\mu}=1 / \sqrt{\left(\frac{\beta_{11}}{\lambda_{1}}\right)^{2}+\left(\frac{\beta_{21}}{\lambda_{2}}\right)^{2}+\left(\frac{\beta_{31}}{\lambda_{3}}\right)^{2}} .
$$


Switching to spherical coordinates

$$
\tilde{\boldsymbol{k}}=\tilde{k} \boldsymbol{\omega}=\tilde{k}(\cos \theta, \sin \theta \cos \psi, \sin \theta \sin \psi)^{T}
$$

the second term within braces of (F.10), evaluated in Appendix H, becomes

$$
\mathcal{E}=\frac{\operatorname{det} \boldsymbol{L}}{\pi^{2} \lambda_{\mu}^{3}} \sum_{m=1}^{\infty} \frac{\sigma_{Y}^{2 m}}{(m-1) !} \int_{z=0}^{\infty} \int_{\theta=0}^{\pi} \int_{\psi=0}^{2 \pi} \boldsymbol{G} \frac{z^{2}}{\left[m^{2}+(B z)^{2}\right]^{2}} \sin \theta d \theta d \psi d z
$$

Here,

$$
\begin{aligned}
& \boldsymbol{G}=\frac{\boldsymbol{Q} Q^{T}}{A^{2}} \quad ; \quad \boldsymbol{Q}=\boldsymbol{\beta} \boldsymbol{\omega} \\
& A^{2}=Q^{T} Q=\omega^{T} \omega \quad ; \quad B^{2}=Q^{T} \zeta^{-2} Q \\
& \boldsymbol{\zeta}^{-2}=\frac{\boldsymbol{L}^{2}}{\lambda_{\mu}^{2}} \quad ; \quad z=\tilde{k} \lambda_{\mu}
\end{aligned}
$$

Equation F.13 after contour integration (Appendix I) and using the transformation $u=\cos \theta$ becomes

$$
\mathcal{E}=\frac{\operatorname{det} \boldsymbol{L}}{4 \pi \lambda_{\mu}^{3}} \sum_{m=1}^{\infty} \frac{\sigma_{Y}^{2 m}}{m !} \int_{u=-1}^{+1} \int_{\psi=0}^{2 \pi} \frac{\boldsymbol{G}}{B^{3}} d u d \psi
$$

The infinite series in (F.17) is written in closed form as

$$
\sum_{m=1}^{\infty} \frac{\sigma_{Y}^{2 m}}{m !}=e^{\sigma_{Y}^{2}}-1
$$

Substituting (F.17)-(F.18) in (F.10) we obtain

$$
K_{e_{i i}}=K_{G} e^{\frac{1}{2} \sigma_{Y}^{2}}\left\{1-\frac{e^{\sigma_{Y}^{2}}-1}{4 \pi \zeta_{1} \zeta_{2} \zeta_{3}} \int_{u=-1}^{+1} \int_{\psi=0}^{2 \pi} \frac{G_{i i}}{B^{3}} d u d \psi\right\}
$$

where $i=1,2,3$.

When the medium is statistically isotropic $\lambda_{\mu}=\lambda_{1}=\lambda_{2}=\lambda_{3}$, regardless of how $\langle\boldsymbol{q}\rangle$ is oriented, and then $\boldsymbol{\beta}=\boldsymbol{\zeta}=\boldsymbol{I}, \boldsymbol{Q}=\boldsymbol{\omega}$ and $A=B=1$. The effective conductivity, equation F.19, now becomes

$$
K_{e_{i i}}=K_{G} e^{\frac{1}{2} \sigma_{Y}^{2}}\left\{1-\frac{e^{\sigma_{Y}^{2}}-1}{4 \pi} \int_{u=-1}^{+1} \int_{\psi=0}^{2 \pi} \boldsymbol{G}_{i i} d u d \psi\right\}
$$


where $\boldsymbol{G}=\boldsymbol{\omega} \boldsymbol{\omega}^{T}$. We can evaluate now the integrals of (F.20),

$$
\begin{aligned}
I_{11} & =\int_{u=-1}^{+1} \int_{\psi=0}^{2 \pi} G_{11} d u d \psi \\
& =\int_{u=-1}^{+1} \int_{\psi=0}^{2 \pi} u^{2} d u d \psi=\frac{4 \pi}{3} \\
I_{22} & =\int_{u=-1}^{+1} \int_{\psi=0}^{2 \pi} G_{22} d u d \psi \\
& =\int_{u=-1}^{+1}\left(1-u^{2}\right) d u \int_{\psi=0}^{2 \pi} \cos ^{2} \psi d \psi \\
& =\frac{4}{3} \int_{\psi=0}^{2 \pi} \cos ^{2} \psi d \psi=\frac{4 \pi}{3},
\end{aligned}
$$

and similarly $I_{33}=4 \pi / 3$. Substituting the value of $4 \pi / 3$ in (F.20) we obtain

$$
K_{e}=\frac{K_{G}}{3} e^{\frac{\sigma_{Y}^{2}}{2}}\left(4-e^{\sigma_{Y}^{2}}\right)
$$




\section{APPENDIX G}

\section{3-DIMENSIONAL FOURIER TRANSFORM OF EXPONENTIAL COVARIANCE}

Using the transformation $\boldsymbol{r}=\boldsymbol{L}^{-\mathbf{1}} \boldsymbol{\eta}$ the exponential covariance in (3.32) becomes isotropic, $C_{Y}(r)=\sigma_{Y}^{2} e^{-r}$. Here, $\boldsymbol{\eta}=\boldsymbol{\chi}-\boldsymbol{x}$, and $\boldsymbol{L}$ is a diagonal matrix with components $\lambda_{1}, \lambda_{2}, \lambda_{3}$, the integral scales in the $\chi_{1}, \chi_{2}, \chi_{3}$ directions, respectively. The three-dimensional FT of the covariance function with the use of the transformation $\boldsymbol{w}=\boldsymbol{L} \boldsymbol{k}$ becomes

$$
\begin{aligned}
F T^{(3)}(-\boldsymbol{w}) & =\frac{1}{(2 \pi)^{\frac{3}{2}}} \int_{\boldsymbol{\chi}} C_{Y}(\boldsymbol{\eta}) e^{-i \boldsymbol{k} \cdot \boldsymbol{\chi}} d \boldsymbol{\chi} \\
& =\frac{1}{(2 \pi)^{\frac{3}{2}}} e^{-i \boldsymbol{k} \cdot \boldsymbol{x}} \int_{\boldsymbol{\eta}} C_{Y}(\boldsymbol{\eta}) e^{-i \boldsymbol{k} \cdot \boldsymbol{\eta}} d \boldsymbol{\eta} \\
& =\operatorname{det}(\mathbf{L}) \frac{\sigma_{Y}^{2}}{(2 \pi)^{\frac{3}{2}}} e^{-i \boldsymbol{k} \cdot \boldsymbol{x}} \int_{\boldsymbol{r}} e^{-r} e^{-i \boldsymbol{w} \cdot \boldsymbol{r}} d \boldsymbol{r},
\end{aligned}
$$

where $\operatorname{det}(\boldsymbol{L})$ is the determinant of matrix $\boldsymbol{L}$. Switching to spherical coordinates

$$
\boldsymbol{r}=r \boldsymbol{\sigma}=r(\cos \theta, \sin \theta \cos \psi, \sin \theta \sin \psi)^{T},
$$

equation G.1 is written as

$$
\begin{array}{r}
F T^{(3)}(-\boldsymbol{w})=\operatorname{det}(\mathbf{L}) \frac{\sigma_{Y}^{2}}{(2 \pi)^{\frac{3}{2}}} e^{-i \boldsymbol{k} \cdot \boldsymbol{x}} \int_{r=0}^{+\infty} \int_{\theta=0}^{\pi} \int_{\psi=0}^{2 \pi} \\
e^{-r} e^{-i w r \cos \theta} r^{2} \sin \theta d \theta d r d \psi,
\end{array}
$$


with $\theta$ the angle between vectors $\boldsymbol{w}$ and $\boldsymbol{r}$. Here, without loss of generality, vector $\boldsymbol{w}$ was taken in the direction of the principal axis $r_{1}$ and the other two axes were rotated accordingly. From Gradshteyn and Ryzhik [1980, p. 482[5] and 966[8.464.1]] we have

$$
\int_{\theta=0}^{\pi} e^{-i w r \cos \theta} \sin \theta d \theta=\frac{2}{w r} \sin (w r),
$$

and then (G.3) becomes

$$
F T^{(3)}(-\boldsymbol{w})=\operatorname{det}(\mathbf{L}) \frac{\sigma_{Y}^{2}}{w} e^{-i \boldsymbol{k} \boldsymbol{x}} \sqrt{\frac{2}{\pi}} \int_{r=0}^{+\infty} r \sin (w r) e^{-r} d r .
$$

From Gradshteyn and Ryzhik [1980, p. 198[5]] we have the following relationship

$$
\begin{aligned}
\int x e^{a x} \sin b x d x=\frac{e^{a x}}{a^{2}+b^{2}} & \\
& {\left[\left(a x-\frac{a^{2}-b^{2}}{a^{2}+b^{2}}\right) \sin b x-\right.} \\
& \left.\left(b x-\frac{2 a b}{a^{2}+b^{2}}\right) \cos b x\right] .
\end{aligned}
$$

Evaluating the integral term in (G.5) with (G.6) $(a=-1$ and $b=w)$ gives us the spectrum of the covariance function

$$
F T^{(3)}(-\boldsymbol{k})=\sqrt{\frac{8}{\pi}} \operatorname{det}(\mathbf{L}) \sigma_{Y}^{2} e^{-i \boldsymbol{k} \boldsymbol{x}} \frac{1}{\left[1+\left(\lambda_{1} k_{1}\right)^{2}+\left(\lambda_{2} k_{2}\right)^{2}+\left(\lambda_{3} k_{3}\right)^{2}\right]^{2}} .
$$

The FT of the m-power of the covariance function is given by

$$
F T_{(m)}^{(3)}(-\boldsymbol{w})=\frac{1}{(2 \pi)^{\frac{3}{2}}} \int_{\boldsymbol{\chi}} C_{Y}^{m}(\boldsymbol{\eta}) e^{-i \boldsymbol{k} \cdot \boldsymbol{\chi}} d \boldsymbol{\chi}
$$

where $C_{Y}^{m}(r)=\sigma_{Y}^{2 m} e^{-m r}$. Equation G.8 in spherical coordinates is written as

$$
\begin{array}{r}
F T_{(m)}^{(3)}(-\boldsymbol{w})=\operatorname{det}(\mathbf{L}) \frac{\sigma_{Y}^{2 m}}{(2 \pi)^{\frac{3}{2}}} e^{-i \boldsymbol{k} \cdot \boldsymbol{x}} \int_{r=0}^{+\infty} \int_{\theta=0}^{\pi} \int_{\psi=0}^{2 \pi} \\
e^{-m r} e^{-i \omega r \cos \theta} r^{2} \sin \theta d \theta d r d \psi,
\end{array}
$$


and based on (G.4) and (G.6) ( $a=-m$ and $b=w)$ it becomes

$$
F T_{(m)}^{(3)}(-\boldsymbol{k})=\sqrt{\frac{8}{\pi}} \operatorname{det}(\mathbf{L}) \sigma_{Y}^{2 m} e^{-i \boldsymbol{k} \cdot \boldsymbol{x}} \frac{m}{\left[m^{2}+\left(\lambda_{1} k_{1}\right)^{2}+\left(\lambda_{2} k_{2}\right)^{2}+\left(\lambda_{3} k_{3}\right)^{2}\right]^{2}}
$$

where $m=1,2, \ldots$ 


\section{APPENDIX H}

\section{EVALUATION OF INTEGRAL TERM OF EQUATION F.10}

We consider the second term within braces of (F.10)

$$
\mathcal{E}=\frac{1}{(2 \pi)^{\frac{3}{2}}} \int_{\boldsymbol{k}} \frac{\boldsymbol{k} \boldsymbol{k}^{T}}{k^{2}} \Phi_{I}(-\boldsymbol{k}) d \boldsymbol{k}
$$

with $\Phi_{I}(-\boldsymbol{k})$ given by

$$
\Phi_{I}(-\boldsymbol{k})=\sqrt{\frac{8}{\pi}} \operatorname{det}(\boldsymbol{L}) \sum_{m=1}^{\infty} \frac{\sigma_{Y}^{2 m}}{(m-1) !} \frac{1}{\left[m^{2}+\left(\lambda_{i} k_{i}\right)^{2}\right]^{2}},
$$

and where summation over $i=1,2,3$ is implied. We transform the terms in (H.1) in the $\tilde{\boldsymbol{\chi}}$ coordinate system (defined in Appendix F). Thus, we have

$$
\frac{\boldsymbol{k}^{T}}{k^{2}}=\frac{\boldsymbol{\beta} \tilde{\boldsymbol{k}}(\boldsymbol{\beta} \tilde{\boldsymbol{k}})^{T}}{\tilde{\boldsymbol{k}}^{T} \tilde{\boldsymbol{k}}}
$$

where the relationship $\boldsymbol{\beta}^{T} \boldsymbol{\beta}=\boldsymbol{I}$ was used. Equation H.3, switching to spherical coordinates

$$
\tilde{\boldsymbol{k}}=\tilde{k} \boldsymbol{\omega}=\tilde{k}(\cos \theta, \sin \theta \cos \psi, \sin \theta \sin \psi)^{T},
$$

is written as

$$
\frac{\boldsymbol{k} \boldsymbol{k}^{T}}{k^{2}}=\frac{\boldsymbol{\beta} \boldsymbol{\omega}(\boldsymbol{\beta} \boldsymbol{\omega})^{T}}{\boldsymbol{\omega}^{T} \boldsymbol{\omega}}=\frac{\boldsymbol{Q} \boldsymbol{Q}^{T}}{A^{2}} .
$$

Here, matrix $\boldsymbol{Q}$ and scalar $A^{2}$ are given by

$$
\boldsymbol{Q}=\boldsymbol{\beta} \boldsymbol{\omega} ; A^{2}=\boldsymbol{Q}^{T} \boldsymbol{Q}=\boldsymbol{\omega}^{T} \boldsymbol{\omega}
$$


The norm of vector $\boldsymbol{w}$, defined in Appendix $G$, is now written as

$$
\begin{aligned}
w=\|\boldsymbol{w}\| & =\|\boldsymbol{L} \boldsymbol{k}\| \\
& =\|\boldsymbol{L} \boldsymbol{\beta} \tilde{\boldsymbol{k}}\|=\tilde{k}\|\boldsymbol{L} \boldsymbol{\beta} \boldsymbol{\omega}\| \\
& =\tilde{k}\left(\boldsymbol{Q}^{T} \boldsymbol{L}^{2} \boldsymbol{Q}\right)^{1 / 2} .
\end{aligned}
$$

Using the transformation

$$
\boldsymbol{\zeta}^{-2}=\frac{\boldsymbol{L}^{2}}{\lambda_{\mu}^{2}}
$$

where $\lambda_{\mu}$ was defined in (F.11), w becomes

$$
w=\tilde{k} \lambda_{\mu}\left(\boldsymbol{Q}^{T} \boldsymbol{\zeta}^{-2} \boldsymbol{Q}\right)^{1 / 2}=\tilde{k} \lambda_{\mu} B
$$

Here, we define scalar $B$ as

$$
B^{2}=\boldsymbol{Q}^{T} \boldsymbol{\zeta}^{-2} \boldsymbol{Q}
$$

The explicit forms of $Q, B^{2}$ and $A^{2}$, with the use of $u=\cos \theta$, are as follows

$$
\begin{gathered}
\boldsymbol{Q}=\left(\begin{array}{c}
\beta_{11} u+\beta_{12} \sqrt{1-u^{2}} \cos \psi+\beta_{13} \sqrt{1-u^{2}} \sin \psi \\
\beta_{21} u+\beta_{22} \sqrt{1-u^{2}} \cos \psi+\beta_{23} \sqrt{1-u^{2}} \sin \psi \\
\beta_{31} u+\beta_{32} \sqrt{1-u^{2}} \cos \psi+\beta_{33} \sqrt{1-u^{2}} \sin \psi
\end{array}\right) \\
B^{2}=Q_{1}^{2}\left(\frac{\lambda_{1}}{\lambda_{\mu}}\right)^{2}+Q_{2}^{2}\left(\frac{\lambda_{2}}{\lambda_{\mu}}\right)^{2}+Q_{3}^{2}\left(\frac{\lambda_{3}}{\lambda_{\mu}}\right)^{2} \\
A^{2}=Q_{1}^{2}+Q_{2}^{2}+Q_{3}^{2} .
\end{gathered}
$$

Substituting (H.2) in (H.1), and using (H.5) and (H.9) we obtain

$$
\mathcal{E}=\frac{\operatorname{det} \boldsymbol{L}}{\pi^{2}} \sum_{m=1}^{\infty} \frac{\sigma_{Y}^{2 m}}{(m-1) !} \int_{\tilde{k}=0}^{\infty} \int_{\theta=0}^{\pi} \int_{\psi=0}^{2 \pi} \boldsymbol{G} \frac{\tilde{k}^{2}}{\left[m^{2}+\left(\tilde{k} \lambda_{\mu} B\right)^{2}\right]^{2}} \sin \theta d \theta d \psi d \tilde{k}
$$

Here, the relationship

$$
G=\frac{Q Q^{T}}{A^{2}}
$$

and the fact that $d \boldsymbol{k}=d \tilde{\boldsymbol{k}}$ were used. 


\section{APPENDIX I}

\section{DERIVATION OF EQUATION F.17}

Integral

$$
I_{1}=\int_{x=0}^{\infty} \frac{x^{2} d x}{\left[m^{2}+(x B)^{2}\right]^{2}},
$$

using the transformation $\alpha=m / B$ becomes

$$
I_{1}=\frac{1}{B^{4}} \int_{x=0}^{\infty} \frac{x^{2} d x}{\left(x^{2}+\alpha^{2}\right)^{2}} .
$$

This integral is evaluated now with the use of residue theorem. Let us consider the integral

$$
I_{2}=\oint_{C} \frac{z^{2} d z}{\left(z^{2}+\alpha^{2}\right)^{2}},
$$

where $C$ is the closed contour consisting of the semicircle $\Gamma$, with radius $R$, and the line from $-R$ to $R$, and where $C$ is traversed in the positive (counterclockwise) sense. The only pole inside this area is the $z=i \alpha$ (of order 2) which has a residue of

$$
\lim _{z \rightarrow i \alpha} \frac{d}{d z}\left[(z-i \alpha)^{2} \frac{z^{2}}{(z+i \alpha)^{2}(z-i \alpha)^{2}}\right]=\frac{1}{4 i \alpha} .
$$

Thus,

$$
\oint_{C} \frac{z^{2} d z}{\left(z^{2}+\alpha^{2}\right)^{2}}=2 \pi i \frac{1}{4 i \alpha}=\frac{\pi}{2 \alpha}
$$

or explicitly

$$
\int_{-R}^{R} \frac{x^{2} d x}{\left(x^{2}+\alpha^{2}\right)^{2}}+\int_{\Gamma} \frac{z^{2} d z}{\left(z^{2}+\alpha^{2}\right)^{2}}=\frac{\pi}{2 \alpha} .
$$


Taking the limit $R \rightarrow \infty$ of (I.6) we obtain

$$
\lim _{R \rightarrow \infty} \int_{-R}^{R} \frac{x^{2} d x}{\left(x^{2}+\alpha^{2}\right)^{2}}=\int_{-\infty}^{+\infty} \frac{x^{2} d x}{\left(x^{2}+\alpha^{2}\right)^{2}}=\frac{\pi}{2 \alpha},
$$

and thus integral $I_{1}$ is given by

$$
I_{1}=\frac{\pi}{4 m B^{3}} .
$$

To proceed from (I.6) to (I.7) we need to show that

$$
\lim _{R \rightarrow \infty} \int_{\Gamma} \frac{z^{2} d z}{\left(z^{2}+\alpha^{2}\right)^{2}}=0 .
$$

Let $z=R e^{i \theta}$, and let us also represent the integrand of expression I.9 by $f(z)$. Then we have

$$
|f(z)|=\left|\frac{R^{2} e^{2 i \theta}}{\left(R^{2} e^{2 i \theta}+\alpha^{2}\right)^{2}}\right| \leq\left|\frac{1}{R^{2} e^{2 i \theta}}\right| \leq \frac{1}{R^{2}} .
$$

Using the property $\left|\oint_{C} f(z) d z\right| \leq M L$, where $M$ is an upper bound of the function $f(z)$ and $L$ is the length of the curve $C$, we have that

$$
\left|\int_{\Gamma} \frac{z^{2} d z}{\left(z^{2}+\alpha^{2}\right)^{2}}\right| \leq \frac{\pi}{R}
$$

Taking the limit $R \rightarrow \infty$ of (I.11) completes the proof of (I.9). 


\section{APPENDIX J}

\section{EFFECTIVE CONDUCTIVITY IN TWO-DIMENSIONAL INFINITE DOMAINS}

The linearized (to first order in $\sigma_{Y}^{2}$ ) $\boldsymbol{K}_{e}$, equation 3.36 , is given by

$$
\boldsymbol{K}_{e}=K_{G}\left\{\left(1+\frac{\sigma_{Y}^{2}}{2}\right) \boldsymbol{I}-\int_{\Omega_{\infty}} C_{Y}(\boldsymbol{\chi}-\boldsymbol{x}) \nabla_{x} \nabla_{\chi}^{T} G_{F}(\boldsymbol{\chi}, \boldsymbol{x}) d \boldsymbol{\chi}\right\} .
$$

Here, $G_{F}(\boldsymbol{\chi}, \boldsymbol{x})$ satisfies the two-dimensional Poisson equation 3.34 and is given by $G_{F}(\boldsymbol{\chi}, \boldsymbol{x})=1 / 2 \pi \ln \eta$ [Greenberg, 1978]. We take $C_{Y}(\boldsymbol{\chi}-\boldsymbol{x})$ to be a two-dimensional anisotropic exponential function

$$
C_{Y}(\boldsymbol{\eta})=\sigma_{Y}^{2} \exp \left[-\left\{\frac{\eta_{1}^{2}}{\lambda_{1}^{2}}+\frac{\eta_{2}^{2}}{\lambda_{2}^{2}}\right\}^{\frac{1}{2}}\right],
$$

where $\boldsymbol{\eta}=\boldsymbol{\chi}-\boldsymbol{x}$ and $\lambda_{1}, \lambda_{2}$ are principal integral scales in the $\chi_{1}, \chi_{2}$ directions, respectively.

Defining the two-dimensional functionals

$$
\begin{gathered}
\Phi^{(2)}(-\boldsymbol{k})=\frac{1}{2 \pi} \int_{-\infty}^{+\infty} \int_{-\infty}^{+\infty} C_{Y}(\boldsymbol{\eta}) e^{-i \boldsymbol{k} \cdot \boldsymbol{\chi}} d \boldsymbol{\chi} \\
\Psi^{(2)}(\boldsymbol{k})=\frac{1}{2 \pi} \int_{-\infty}^{+\infty} \int_{-\infty}^{+\infty} \nabla_{x} \nabla_{\chi}^{T} G_{F}(\boldsymbol{\chi}, \boldsymbol{x}) e^{i \boldsymbol{k} \cdot \boldsymbol{\chi}} d \boldsymbol{\chi}
\end{gathered}
$$

and using Parseval's identity (B.9), equation J.1 becomes

$$
\boldsymbol{K}_{e}=K_{G}\left\{\left(1+\frac{\sigma_{Y}^{2}}{2}\right) \boldsymbol{I}-\int_{\boldsymbol{k}} \Phi^{(2)}(-\boldsymbol{k}) \Psi^{(2)}(\boldsymbol{k}) d \boldsymbol{k}\right\}
$$


where $\boldsymbol{k}=\left(k_{1}, k_{2}\right)^{T}$ is a two-dimensional wave number vector in the Cartesian coordinates $\chi=\left(\chi_{1}, \chi_{2}\right)^{T}$ which are oriented parallel to the principal directions of $\log$ hydraulic conductivity.

Taking the Fourier transform (FT) of (3.34), Appendix C, allows expressing $(\mathrm{J} .4)$ as

$$
\Psi^{(2)}(\boldsymbol{k})=\Psi^{\prime}(\boldsymbol{k}) e^{i \boldsymbol{k} \cdot \boldsymbol{x}} \quad \text { where } \quad \Psi^{\prime}(\boldsymbol{k})=\frac{1}{2 \pi} \frac{\boldsymbol{k} \boldsymbol{k}^{T}}{k^{2}} .
$$

Defining the transformations $\boldsymbol{\chi}^{*}=\boldsymbol{L}^{-1} \boldsymbol{\eta}$ and $\boldsymbol{k}^{*}=\boldsymbol{L} \boldsymbol{k}$, where $\boldsymbol{L}$ is a $2 \times 2$ diagonal matrix with non-zero elements $\lambda_{1}$ and $\lambda_{2}$, renders the covariance in (J.2) isotropic. Its two-dimensional FT in (J.3) then becomes

$$
\begin{aligned}
\Phi^{(2)}\left(-\boldsymbol{k}^{*}\right) & =\frac{\sigma_{Y}^{2}}{2 \pi} e^{-i \boldsymbol{k} \cdot \boldsymbol{x}} \int_{\boldsymbol{\eta}} e^{-\chi^{*}} e^{-i \boldsymbol{k} \cdot \boldsymbol{\eta}} d \boldsymbol{\eta} \\
& =\frac{\lambda_{1} \lambda_{2} \sigma_{Y}^{2}}{2 \pi} e^{-i \boldsymbol{k} \cdot \boldsymbol{x}} \int_{\boldsymbol{\chi}^{*}} e^{-\chi^{*}} e^{-i \boldsymbol{k}^{*} \cdot \boldsymbol{\chi}^{*}} d \boldsymbol{\chi}^{*}
\end{aligned}
$$

Transforming into polar coordinates we obtain

$$
\Phi^{(2)}\left(-\boldsymbol{k}^{*}\right)=\frac{\lambda_{1} \lambda_{2} \sigma_{Y}^{2}}{2 \pi} e^{-i \boldsymbol{k} \cdot \boldsymbol{x}} \int_{\chi^{*}=0}^{+\infty} \int_{\theta=0}^{2 \pi} e^{-\chi^{*}} e^{-i k^{*} \chi^{*} \cos \theta} \chi^{*} d \chi^{*} d \theta
$$

which with the use of the relationship [Gradshteyn and Ryzhik, 1980, pp. 482[5] and $951[8.402]]$

$$
\int_{\theta=0}^{2 \pi} e^{-i k^{*} \chi^{*} \cos \theta} d \theta=2 \pi J_{0}\left(k^{*} \chi^{*}\right)
$$

becomes

$$
\Phi^{(2)}\left(-\boldsymbol{k}^{*}\right)=\lambda_{1} \lambda_{2} \sigma_{Y}^{2} e^{-i \boldsymbol{k} \cdot \boldsymbol{x}} \int_{\chi^{*}=0}^{+\infty} \chi^{*} e^{-\chi^{*}} J_{0}\left(k^{*} \chi^{*}\right) d \chi^{*}
$$

where $J_{0}\left(k^{*} \chi^{*}\right)$ is Bessel function of order zero. From Erdelyi et al. [1954, pp. $9[20]$ and 424] we find that

$$
\Phi^{(2)}\left(-\boldsymbol{k}^{*}\right)=\lambda_{1} \lambda_{2} \sigma_{Y}^{2} e^{-i \boldsymbol{k} \cdot \boldsymbol{x}} \frac{1}{\left(1+k^{*^{2}}\right)} P_{\mathbf{1}}\left[\frac{1}{\left(1+k^{*^{2}}\right)^{1 / 2}}\right]
$$


where $P_{1}\left(k^{*}\right)$ is the Legendre Polynomial of order 1 . Evaluating $P_{1}\left(k^{*}\right)$, Appendix $\mathrm{D}$, we obtain

$$
\Phi^{(2)}(-\boldsymbol{k})=\lambda_{\mathbf{1}} \lambda_{2} \sigma_{Y}^{2} \frac{1}{\left[1+\left(\lambda_{1} k_{1}\right)^{2}+\left(\lambda_{2} k_{2}\right)^{2}\right]^{3 / 2}} e^{-i \boldsymbol{k} \cdot \boldsymbol{x}}
$$

Substituting (J.6) and (J.12) in (J.5) we obtain

$$
\boldsymbol{K}_{e}=K_{G}\left\{\left(1+\frac{\sigma_{Y}^{2}}{2}\right) \boldsymbol{I}-\frac{\lambda_{1} \lambda_{2} \sigma_{Y}^{2}}{2 \pi} \int_{\boldsymbol{k}} \frac{\boldsymbol{k}^{T}}{k^{2}} \frac{d \boldsymbol{k}}{\left[1+\left(\lambda_{1} k_{1}\right)^{2}+\left(\lambda_{2} k_{2}\right)^{2}\right]^{3 / 2}}\right\}
$$

which is the two-dimensional version of the three-dimensional expression (3.37) of Gutjahr et al. [1978], Gelhar and Axness [1981, 1983a], Neuman and Depner [1988], Dagan [1989], and Kitanidis [1990]. Equation J.13 is identical to that by SanchezVilla and Carrera [1993] who developed it independently following the approach of Dagan [1989].

We consider now a system of coordinates $\tilde{\chi}=\left(\tilde{\chi}_{1}, \tilde{\chi}_{2}\right)^{T}$ where $\tilde{\chi}_{1}$ is parallel to the direction of mean flow. The two-dimensional matrix $\boldsymbol{\beta}$ of direction cosines between $\chi_{i}$ and $\tilde{\chi}_{j}(i, j=1,2)$ is

$$
\boldsymbol{\beta}=\left(\begin{array}{cc}
\cos \theta & -\sin \theta \\
\sin \theta & \cos \theta
\end{array}\right)
$$

where $\theta$ is the angle between $\chi_{1}$ and $\tilde{\chi}_{1}$, and $\lambda_{\mu}$ is the length of a radius vector parallel to $\langle\boldsymbol{q}\rangle$ corresponding to an ellipse having semi-axes $\lambda_{1}$ and $\lambda_{2}$ oriented along $\chi_{1}$ and $\chi_{2}$, respectively. The latter is given by

$$
\lambda_{\mu}=1 / \sqrt{\left(\frac{\cos \theta}{\lambda_{1}}\right)^{2}+\left(\frac{\sin \theta}{\lambda_{2}}\right)^{2}}
$$

We can then write in polar coordinates

$$
\tilde{\boldsymbol{k}}=\tilde{k} \boldsymbol{\omega}=\tilde{k}(\cos \theta, \sin \theta)^{T}
$$


where $\tilde{k}$ is the norm of the wave number vector $\tilde{\boldsymbol{k}}$ corresponding to the $\tilde{\chi}$ coordinates. Upon defining (similar to appendix $\mathrm{F}$ ) the transformations

$$
\begin{array}{r}
\boldsymbol{G}=\boldsymbol{Q} \boldsymbol{Q}^{T} \quad ; \quad \boldsymbol{Q}=\boldsymbol{\beta} \boldsymbol{\omega}=(\cos 2 \theta, \sin 2 \theta)^{T} \\
A^{2}=\boldsymbol{Q}^{T} \boldsymbol{Q}=1 \quad ; \quad B^{2}=\boldsymbol{Q}^{T} \boldsymbol{\zeta}^{-2} \boldsymbol{Q} \\
\boldsymbol{\zeta}^{-2}=\frac{\boldsymbol{L}^{2}}{\lambda_{\mu}^{2}} \quad ; \quad z=\tilde{k} \lambda_{\mu}
\end{array}
$$

the second term within braces in (J.13) becomes

$$
\mathcal{E}=\frac{(\operatorname{det} \mathbf{L}) \sigma_{Y}^{2}}{2 \pi \lambda_{\mu}^{2}} \int_{z=0}^{\infty} \int_{\theta=0}^{2 \pi} G \frac{z}{\left[1+(B z)^{2}\right]^{3 / 2}} d z d \theta
$$

where $\operatorname{det} \mathbf{L}=\lambda_{1} \lambda_{2}$. From Gradshteyn and Ryzhik [1980, p. 83[6]],

$$
\int_{z=0}^{\infty} \frac{z}{\left[1+(B z)^{2}\right]^{3 / 2}} d z=\frac{1}{B^{2}}
$$

so that (J.20) simplifies to

$$
\mathcal{E}=\frac{(\operatorname{det} \mathbf{L}) \sigma_{Y}^{2}}{2 \pi \lambda_{\mu}^{2}} \int_{\theta=0}^{2 \pi} \frac{\boldsymbol{G}}{B^{2}} d \theta
$$

Substituting $G_{11}=\cos ^{2} 2 \theta, B^{2}=\left(\lambda_{1} / \lambda_{\mu}\right)^{2} \cos ^{2} 2 \theta+\left(\lambda_{2} / \lambda_{\mu}\right)^{2} \sin ^{2} 2 \theta, c^{2}=\left(\lambda_{1} / \lambda_{\mu}\right)^{2}$ and $d^{2}=\left(\lambda_{2} / \lambda_{\mu}\right)^{2}$ in $(\mathrm{J} .22)$, we can write

$$
\mathcal{E}_{11}=\frac{(\operatorname{det} \mathbf{L}) \sigma_{Y}^{2}}{\pi \lambda_{\mu}^{2}} \int_{t=0}^{\pi} \frac{\cos ^{2} t}{c^{2} \cos ^{2} t+d^{2} \sin ^{2} t} d t=\frac{\lambda_{2} \sigma_{Y}^{2}}{\lambda_{1}+\lambda_{2}} .
$$

This result was obtained with the aid of Macsyma [1992] by making the change of variable $t=2 \theta$ and recognizing that the integrand is an even function. By the same token we find immediately that

$$
\mathcal{E}_{22}=\frac{\lambda_{1} \sigma_{Y}^{2}}{\lambda_{1}+\lambda_{2}}
$$


whereas the off-diagonal terms $\mathcal{E}_{12}$ and $\mathcal{E}_{21}$, by virtue of the integration of an odd function over a symmetric interval, equal zero. An alternative evaluation of the $\mathcal{E}$ term, due to Sanchez-Villa and Carrera [1993], is provided in Appendix E for reasons of completeness. This appendix applies, for the two-dimensional case, the approach of Gelhar and Axness [1981, Appendix G, 1983a], and Dagan [1989] for the evaluation of the three-dimensional $\boldsymbol{K}_{e}$ from (3.37).

Upon substitution of (J.23) and (J.24) in (J.13) the linearized form for the principal components of the effective conductivity becomes

$$
\begin{aligned}
K_{e_{i i}}=K_{G}\left[1+\frac{\sigma_{Y}^{2}}{2}-\frac{\lambda_{j} \sigma_{Y}^{2}}{\lambda_{1}+\lambda_{2}}\right] \quad & i, j=1,2 \\
& \text { and } i \neq j
\end{aligned}
$$

which is identical to (2.33), derived from the relationships of Gelhar and Axness [1983a], and coincides with the result of Sanchez-Villa and Carrera [1993]. Invoking the Landau-Lifshitz [1960] conjecture we finally obtain, equation 2.35,

$$
\begin{array}{r}
K_{e_{i i}}=K_{G} \exp \left[\sigma_{Y}^{2}\left(\frac{1}{2}-\frac{\lambda_{j}}{\lambda_{1}+\lambda_{2}}\right)\right] \quad \begin{array}{r}
i, j=1,2 \\
\text { and } i \neq j
\end{array}
\end{array}
$$

which is valid for large $\sigma_{Y}^{2}$. 


\section{APPENDIX K}

\section{NUMERICAL EVALUATION USING GAUSSIAN QUADRATURE}

Let us consider integral

$$
I I=\int_{\psi=0}^{d} \int_{u=-1}^{+1} f(u, \psi) d u d \psi
$$

The Gauss-Legendre formula for an integral evaluated at any finite interval $[a, b]$ is given by

$$
\int_{a}^{b} f(t) d t \approx \frac{b-a}{2} \sum_{k=1}^{n} w_{k} f\left(\frac{b-a}{2} x_{k}+\frac{a+b}{2}\right),
$$

where $w_{k}$ and $x_{k}$ are the Gauss weights and points respectively, and $\mathrm{n}$ is the total number of Gauss points. By rewriting (K.1) as

$$
I I=\int_{\psi=0}^{d}\left[\int_{u=-1}^{0} f(u, \psi) d u+\int_{u=0}^{+1} f(u, \psi) d u\right] d \psi,
$$

the integral over $u$ can be approximated, using the Gauss-Legendre formula, by

$$
I I \approx \frac{1}{2} \int_{\psi=0}^{d}\left[\sum_{k=1}^{n / 2} w_{k}\left[f\left(u_{k}^{1}, \psi\right)+f\left(-u_{k}^{2}, \psi\right)+f\left(u_{k}^{2}, \psi\right)+f\left(-u_{k}^{1}, \psi\right)\right]\right] d \psi .
$$

Here,

$$
\begin{aligned}
& u_{k}^{1}=\frac{1}{2}\left(x_{k}-1\right) \\
& u_{k}^{2}=\frac{1}{2}\left(x_{k}+1\right),
\end{aligned}
$$


and where the symmetricity over zero in the $[-1,+1]$ interval of the Gauss points was utilized. Applying the Gauss-Legendre formula to the integral over $\psi$ we obtain

$$
\begin{aligned}
I I=\frac{d}{4} \sum_{l=1}^{m / 2} \sum_{k=1}^{n / 2} \quad & w_{l} w_{k}\left[f\left(u_{k}^{1}, \psi_{l}^{1}\right)+f\left(-u_{k}^{2}, \psi_{l}^{1}\right)+f\left(u_{k}^{2}, \psi_{l}^{1}\right)+\right. \\
& f\left(-u_{k}^{1}, \psi_{l}^{1}\right)+f\left(u_{k}^{1}, \psi_{l}^{2}\right)+f\left(-u_{k}^{2}, \psi_{l}^{2}\right)+f\left(u_{k}^{2}, \psi_{l}^{2}\right)+ \\
& \left.f\left(-u_{k}^{1}, \psi_{l}^{2}\right)\right]
\end{aligned}
$$

where now,

$$
\begin{gathered}
\psi_{l}^{1}=\frac{d}{2}\left(x_{l}+1\right) \\
\psi_{l}^{2}=\frac{d}{2}\left(-x_{l}+1\right) .
\end{gathered}
$$




\section{APPENDIX L}

\section{DERIVATION OF EQUATION 4.43}

Let us define

$$
\begin{gathered}
k_{1}^{*}=\lambda_{1} k_{1}, \\
\chi_{1}^{*}=\frac{\chi_{1}-x_{1}}{\lambda_{1}},
\end{gathered}
$$

where $k_{1}$, and $\lambda_{1}$, are, the coordinate of the wave number vector $\boldsymbol{k}$, and the integral length in the $\chi_{1}$-direction, respectively. The three-dimensional FT of the exponential covariance function is given by

$$
\begin{aligned}
& F T^{(3)}\left(-k_{1},-k_{2},-k_{3}\right)= \\
& \quad=\frac{1}{\sqrt{2 \pi}} \int_{\chi_{1}=-\infty}^{+\infty} F T^{(2)}\left(-k_{2},-k_{3} ; \chi_{1}\right) e^{-i k_{1} \chi_{1}} d \chi_{1} \\
& \quad=\frac{\lambda_{1}}{\sqrt{2 \pi}} e^{-i k_{1} x_{1}} \int_{\chi_{1}^{*}=-\infty}^{+\infty} F T^{(2)}\left(-k_{2},-k_{3} ; \chi_{1}^{*}\right) e^{-i k_{1}^{*} \chi_{1}^{*}} d \chi_{1}^{*} .
\end{aligned}
$$

Substituting in (L.3) the two-dimensional FT of the exponential covariance function, (4.39), we obtain

$$
\begin{aligned}
F T^{(3)}(-\boldsymbol{k})= & e^{-i \boldsymbol{k} \cdot \boldsymbol{x}} \frac{\lambda_{1} \lambda_{2} \lambda_{3}}{\sqrt{2 \pi}} \sigma_{Y}^{2}\left(1+k^{*^{2}}\right)^{-3 / 2} \\
& \int_{-\infty}^{+\infty} e^{-i k_{1}^{*} \chi_{1}^{*}}\left[1+\left|\chi_{1}^{*}\right| \sqrt{1+k^{*^{2}}}\right] e^{-\left|\chi_{1}^{*}\right| \sqrt{1+k^{* 2}}} d \chi_{1}^{*},
\end{aligned}
$$

where

$$
k^{*^{2}}=\left(\lambda_{2} k_{2}\right)^{2}+\left(\lambda_{3} k_{3}\right)^{2} .
$$


Substituting in (L.5) the appropriate expressions for the absolute value $\left|\chi_{1}^{*}\right|$ we obtain

$$
\begin{aligned}
F T^{(3)}(-\boldsymbol{k})= & e^{-i \boldsymbol{k} \cdot \boldsymbol{x}} \frac{\lambda_{1} \lambda_{2} \lambda_{3}}{\sqrt{2 \pi}} \sigma_{Y}^{2}\left(1+k^{*^{2}}\right)^{-3 / 2} \\
& \left\{\int_{0}^{+\infty} e^{-i k_{1}^{*} \chi_{1}^{*}}\left[1+\chi_{1}^{*} \sqrt{1+k^{*^{2}}}\right] e^{-\chi_{1}^{*} \sqrt{1+k^{* 2}}} d \chi_{1}^{*}\right. \\
& \left.+\int_{0}^{+\infty} e^{+i k_{1}^{*} \chi_{1}^{*}}\left[1+\chi_{1}^{*} \sqrt{1+k^{*^{2}}}\right] e^{-\chi_{1}^{*} \sqrt{1+k^{*}}} d \chi_{1}^{*}\right\}
\end{aligned}
$$

With the use of the transformation

$$
\beta=\sqrt{1+k^{*^{2}}}
$$

equation L. 6 becomes

$$
\begin{aligned}
F T^{(3)}(-\boldsymbol{k})= & e^{-i \boldsymbol{k} \cdot \boldsymbol{x}} \frac{\lambda_{1} \lambda_{2} \lambda_{3}}{\sqrt{2 \pi}} \sigma_{Y}^{2}\left(1+k^{*^{2}}\right)^{-3 / 2}\left\{\int_{0}^{+\infty} e^{-\chi_{1}^{*}\left(\beta+i k_{1}^{*}\right)} d \chi_{1}^{*}\right. \\
& +\beta \int_{0}^{+\infty} \chi_{1}^{*} e^{-\chi_{1}^{*}\left(\beta+i k_{1}^{*}\right)} d \chi_{1}^{*}+\int_{0}^{+\infty} e^{-\chi_{1}^{*}\left(\beta-i k_{1}^{*}\right)} d \chi_{1}^{*} \\
& \left.+\beta \int_{0}^{+\infty} \chi_{1}^{*} e^{-\chi_{1}^{*}\left(\beta-i k_{1}^{*}\right)} d \chi_{1}^{*}\right\}
\end{aligned}
$$

The exponential integrals of (L.8) can be easily calculated to produce

$$
\begin{aligned}
F T^{(3)}(-\boldsymbol{k})= & e^{-i \boldsymbol{k} \cdot \boldsymbol{x} \frac{\lambda_{1} \lambda_{2} \lambda_{3}}{\sqrt{2 \pi}} \sigma_{Y}^{2}\left(1+k^{*^{2}}\right)^{-3 / 2}} \\
& \left\{\frac{1}{\beta+i k_{1}^{*}}+\frac{1}{\beta-i k_{1}^{*}}+\frac{\beta}{\left(\beta+i k_{1}^{*}\right)^{2}}+\frac{\beta}{\left(\beta-i k_{1}^{*}\right)^{2}}\right\}
\end{aligned}
$$

which after some algebraic manipulations of the terms within braces results to

$$
F T^{(3)}(-\boldsymbol{k})=\sqrt{\frac{8}{\pi}} \lambda_{1} \lambda_{2} \lambda_{3} \frac{\sigma_{Y}^{2}}{\left(\beta^{2}+k_{1}^{*^{2}}\right)^{2}} e^{-i \boldsymbol{k} \cdot \boldsymbol{x}},
$$

the three-dimensional FT of the exponential covariance function. 


\section{APPENDIX M}

\section{SOLUTION OF EQUATION 4.53}

Let us consider equation

$$
-\frac{d^{2} u}{d \chi^{2}}+\beta^{2} u=\delta(\chi-x),
$$

satisfying the boundary conditions

$$
u(-\infty, x)=0 \quad, \quad u(+\infty, x)=0,
$$

and where $\beta^{2}$ is a constant. The general solution of (M.1) is given by

$$
u(\chi, x)=\left\{\begin{array}{lll}
A e^{\beta \chi}+B e^{-\beta \chi} & \text { for } & -\infty<\chi<x \\
C e^{\beta \chi}+D e^{-\beta \chi} & \text { for } & x<\chi<+\infty
\end{array}\right.
$$

where $A, B, C$, and $D$, constant coefficients to be determined subsequently. From the boundary conditions (M.2) it follows that the constants $B=C=0$, and then $u(\chi, x)$ becomes

$$
u(\chi, x)=\left\{\begin{array}{lll}
A e^{\beta \chi} & \text { for } & -\infty<\chi<x \\
D e^{-\beta \chi} & \text { for } & x<\chi<+\infty
\end{array}\right.
$$

In order for the function $u(\chi, x)$ to be continuous, at $\chi=x$ we must have

$$
A e^{\beta x}=D e^{-\beta x},
$$

which is the first equation relating the unknown coefficients $A$, and $D$. The second relationship is obtained by integrating (M.1) across the singularity $x$, that is

$$
\begin{aligned}
-\int_{x-0}^{x+0}\left(u_{\chi \chi}-\beta^{2} u\right) d \chi=\int_{x-0}^{x+0} \delta(\chi-x) d \chi & =1 \\
-\left.\frac{d u}{d \chi}\right|_{x-0} ^{x+0}+\beta^{2} \int_{x-0}^{x+0} u d \chi & =1 \\
\beta D e^{-\beta x}+\beta A e^{\beta x} & =1
\end{aligned}
$$


and where the integral over $u(\chi, x)$ was set equal to zero from the requirement of continuity of $u(\chi, x)$ at $\chi=x$. Solving the system of equations (M.5) and (M.6) for $A$ and $D$, we obtain the solution of (M.1) subject to (M.2),

$$
u(\chi, x)=\left\{\begin{array}{lll}
\frac{1}{2 \beta} e^{-\beta(x-\chi)} & \text { for } & -\infty<\chi<x \\
\frac{1}{2 \beta} e^{-\beta(\chi-x)} & \text { for } & x<\chi<+\infty
\end{array}\right.
$$

or in a compact form

$$
u(\chi, x)=\frac{1}{2 \beta} e^{-\beta|\chi-x|}
$$

The solution of (M.1) subject to the boundary conditions

$$
u(-a, x)=0 \quad, \quad u(+a, x)=0
$$

is obtained (utilizing (M.8)) with the method of images [Stakgold, 1979], and is given by

$$
u(\chi, x)=\frac{1}{2 \beta} \sum_{j=-\infty}^{+\infty}(-1)^{j} e^{-\beta\left|\chi-2 j a-(-1)^{j} x\right|}
$$




\section{APPENDIX N \\ ONE-DIMENSIONAL FT OF EQUATION 4.67}

The two-dimensional FT, with respect to $\chi_{2}$ and $\chi_{3}$, of the second mixed derivative of the infinite Green's function is given from (4.67) as

$$
\begin{aligned}
\hat{g}_{x_{1} \chi_{1}}= & \frac{1}{2 \pi} e^{i\left(k_{2} x_{2}+k_{3} x_{3}\right)}\left\{\delta\left(\chi_{1}-x_{1}\right)\right. \\
& \left.-\frac{\left(k_{2}^{2}+k_{3}^{2}\right)^{1 / 2}}{2} e^{-\left(k_{2}^{2}+k_{3}^{2}\right)^{1 / 2}\left|\chi_{1}-x_{1}\right|}\right\} .
\end{aligned}
$$

We set

$$
\chi_{1}^{*}=\chi_{1}-x_{1},
$$

and then a three-dimensional FT is given by

$$
\begin{aligned}
F T^{(3)}(\boldsymbol{k}, \boldsymbol{x}) & =\frac{1}{\sqrt{2 \pi}} \int_{\chi_{1}=-\infty}^{+\infty} F T^{(2)}\left(k_{2}, k_{3} ; \chi_{1}, \boldsymbol{x}\right) e^{i k_{1} \chi_{1}} d \chi_{1} \\
& =\frac{e^{i k_{1} x_{1}}}{\sqrt{2 \pi}} \int_{\chi_{1}^{*}=-\infty}^{+\infty} F T^{(2)}\left(k_{2}, k_{3} ; \chi_{1}^{*}, x_{2}, x_{3}\right) e^{i k_{1} \chi_{1}^{*}} d \chi_{1}^{*} .
\end{aligned}
$$

Substituting (N.1) into (N.3) we obtain

$$
F T^{(3)}(\boldsymbol{k}, \boldsymbol{x})=\frac{e^{i \boldsymbol{k} \cdot \boldsymbol{x}}}{(2 \pi)^{3 / 2}}\left\{1-\frac{k^{\prime}}{2} \int_{-\infty}^{+\infty} e^{i k_{1} \chi_{1}^{*}} e^{-k^{\prime}\left|\chi_{1}^{*}\right|} d \chi_{1}^{*}\right\}
$$

where $k^{\prime 2}=k_{2}^{2}+k_{3}^{2}$. Substituting the appropriate values of $\left|\chi_{1}^{*}\right|$, (N.4) becomes

$$
\begin{aligned}
F T^{(3)}(\boldsymbol{k}, \boldsymbol{x})= & \frac{e^{i \boldsymbol{k} \cdot \boldsymbol{x}}}{(2 \pi)^{3 / 2}}\left\{1-\frac{k^{\prime}}{2} \int_{0}^{+\infty} e^{-\chi_{1}^{*}\left(k^{\prime}-i k_{1}\right)} d \chi_{1}^{*}\right. \\
& \left.-\frac{k^{\prime}}{2} \int_{0}^{+\infty} e^{-\chi_{1}^{*}\left(k^{\prime}+i k_{1}\right)} d \chi_{1}^{*}\right\} .
\end{aligned}
$$


Calculating the exponential integrals of (N.5) results in

$$
F T^{(3)}(\boldsymbol{k}, \boldsymbol{x})=\frac{e^{i \boldsymbol{k} \cdot \boldsymbol{x}}}{(2 \pi)^{3 / 2}}\left\{1-\frac{k^{\prime}}{2} \frac{2 k^{\prime}}{\left(k^{\prime 2}+k_{1}^{2}\right)}\right\},
$$

which, upon the substitution of $k^{\prime 2}=k_{2}^{2}+k_{3}^{2}$, gives

$$
F T^{(3)}(\boldsymbol{k}, \boldsymbol{x})=\frac{e^{i \boldsymbol{k} \cdot \boldsymbol{x}}}{(2 \pi)^{3 / 2}} \frac{k_{1}^{2}}{k_{1}^{2}+k_{2}^{2}+k_{3}^{2}}
$$

the three-dimensional FT of the mixed derivatives of the fundamental solution (Appendix C). 


\section{APPENDIX O}

\section{DERIVATION OF THE SUM OF THE SERIES IN 4.66}

Let us commence by expanding the series of (4.65),

$$
\begin{aligned}
& \sum_{j=-\infty}^{+\infty} e^{-k^{\prime}\left|\chi_{1}-2 j a-(-1)^{j} x_{1}\right|}=e^{-k^{\prime}\left|\chi_{1}-x_{1}\right|}+ \\
& \quad+\sum_{j=1}^{+\infty} e^{-k^{\prime}\left|\chi_{1}-2 j a-(-1)^{j} x_{1}\right|}+\sum_{j=1}^{+\infty} e^{-k^{\prime}\left|\chi_{1}+2 j a-(-1)^{j} x_{1}\right|} .
\end{aligned}
$$

Now, the following relationships are always satisfied

$$
\begin{aligned}
& \chi_{1}-2 j a-(-1)^{j} x_{1}<0 \\
& \chi_{1}+2 j a-(-1)^{j} x_{1}>0 .
\end{aligned}
$$

For $j=$ odd, say $j=2 \rho-1$, with $\rho=1,2, \cdots,+\infty$, and using (O.2) and (O.3) we can write

$$
\begin{aligned}
& e^{-k^{\prime}\left|\chi_{1}-2 j a-(-1)^{j} x_{1}\right|}+e^{-k^{\prime}\left|\chi_{1}+2 j a-(-1)^{j} x_{1}\right|}= \\
& =e^{+k^{\prime}\left(\chi_{1}-2 j a+x_{1}\right)}+e^{-k^{\prime}\left(\chi_{1}+2 j a+x_{1}\right)}= \\
& =e^{-2 k^{\prime} a j}\left[e^{k^{\prime}\left(\chi_{1}+x_{1}\right)}+e^{-k^{\prime}\left(\chi_{1}+x_{1}\right)}\right]= \\
& =2 \cosh \left[k^{\prime}\left(\chi_{1}+x_{1}\right)\right] e^{-2 k^{\prime} a j} .
\end{aligned}
$$

For $j=$ even, say $j=2 \rho, \rho=1,2, \cdots,+\infty$, we obtain

$$
e^{-k^{\prime}\left|x_{1}-2 j a-(-1)^{j} x_{1}\right|}+e^{-k^{\prime}\left|\chi_{1}+2 j a-(-1)^{j} x_{1}\right|}=
$$




$$
\begin{aligned}
& =e^{+k^{\prime}\left(\chi_{1}-2 j a-x_{1}\right)}+e^{-k^{\prime}\left(\chi_{1}+2 j a-x_{1}\right)}= \\
& =e^{-2 k^{\prime} a j}\left[e^{k^{\prime}\left(\chi_{1}-x_{1}\right)}+e^{-k^{\prime}\left(\chi_{1}-x_{1}\right)}\right]= \\
& =2 \cosh \left[k^{\prime}\left(\chi_{1}-x_{1}\right)\right] e^{-2 k^{\prime} a j} .
\end{aligned}
$$

For $j=2 \rho-1(j=$ odd $)$ we have that

$$
e^{-2 k^{\prime} a j}=e^{-2 k^{\prime} a(2 \rho-1)}=e^{-4 k^{\prime} a \rho} e^{2 k^{\prime} a},
$$

whereas for $j=2 \rho(j=$ even $)$ we have that

$$
e^{-2 k^{\prime} a j}=e^{-4 k^{\prime} a \rho}
$$

Substituting (0.4) - (O.7) into (O.1) we obtain

$$
\begin{aligned}
& \sum_{j=-\infty}^{+\infty} e^{-k^{\prime}\left|\chi_{1}-2 j a-(-1)^{j} x_{1}\right|}=e^{-k^{\prime}\left|\chi_{1}-x_{1}\right|}+ \\
& \quad+2\left\{e^{2 k^{\prime} a} \cosh \left[k^{\prime}\left(\chi_{1}+x_{1}\right)\right]+\cosh \left[k^{\prime}\left(\chi_{1}-x_{1}\right)\right]\right\} \sum_{\rho=1}^{+\infty} e^{-4 k^{\prime} a \rho} .
\end{aligned}
$$

The partial sum of the series $\sum_{\rho=0}^{+\infty} e^{-4 k^{\prime} a \rho}$ is

$$
S_{n}=1+e^{-4 k^{\prime} a}+\cdots+e^{-4 k^{\prime} a(n-1)},
$$

and multiplying $S_{n}$ by $\exp \left(-4 k^{\prime} a\right)$ we obtain

$$
e^{-4 k^{\prime} a} S_{n}=e^{-4 k^{\prime} a}+\cdots+e^{-4 k^{\prime} a(n-1)}+e^{-4 k^{\prime} a n}
$$

Subtracting (O.10) from (O.9), and taking the limit as $n \rightarrow \infty$ we obtain

$$
\sum_{\rho=0}^{+\infty} e^{-4 k^{\prime} a \rho}=\frac{1}{1-e^{-4 k^{\prime} a} .}
$$

We have that

$$
\sum_{\rho=1}^{+\infty} e^{-4 k^{\prime} a \rho}=-1+\sum_{\rho=0}^{+\infty} e^{-4 k^{\prime} a \rho}
$$


which after the substitution of $(0.11)$, and multiplication and division by $e^{2 k^{\prime} a}$ gives

$$
\sum_{\rho=1}^{+\infty} e^{-4 k^{\prime} a \rho}=\frac{e^{-2 k^{\prime} a}}{2 \sinh \left[2 k^{\prime} a\right]} .
$$

Finally, substituting (O.13) into (O.8) we obtain

$$
\begin{aligned}
& \sum_{j=-\infty}^{+\infty} e^{-k^{\prime}\left|x_{1}-2 j a-(-1)^{j} x_{1}\right|}=e^{-k^{\prime}\left|\chi_{1}-x_{1}\right|}+ \\
& \quad+\frac{\cosh \left[k^{\prime}\left(\chi_{1}+x_{1}\right)\right]}{\sinh \left[2 k^{\prime} a\right]}+e^{-2 k^{\prime} a} \frac{\cosh \left[k^{\prime}\left(\chi_{1}-x_{1}\right)\right]}{\sinh \left[2 k^{\prime} a\right]}
\end{aligned}
$$

which has also been presented in the stochastic literature (without any details of its derivation) by Naff and Vecchia [1986].

We investigate now the convergence behavior of (O.1). Defining the transformations $\rho_{1}=a / \lambda_{1}, \rho_{\chi}=\chi_{1} / a, \rho_{x}=x_{1} / a$, and $z=\lambda_{1} k^{\prime}$, the series of (O.1) is written as

$$
\begin{aligned}
\sum_{j=-\infty}^{+\infty} e^{-k^{\prime}\left|\chi_{1}-2 j a-(-1)^{j} x_{1}\right|} & =\sum_{j=-\infty}^{+\infty} e^{-\alpha k^{\prime}\left|\rho_{\chi}-2 j-(-1)^{j} \rho_{x}\right|} \\
& =\sum_{j=-\infty}^{+\infty} e^{-\rho_{1}\left|\rho_{\chi}-2 j-(-1)^{j} \rho_{x}\right| z} .
\end{aligned}
$$

Expanding the series of (O.15), and using (O.2) and (O.3), we obtain

$$
\begin{aligned}
& \sum_{j=-\infty}^{+\infty} e^{-\rho_{1}\left|\rho_{\chi}-2 j-(-1)^{j} \rho_{x}\right| z}=e^{-\rho_{1}\left|\rho_{\chi}-\rho_{x}\right| z} \\
& \quad+\sum_{j=1}^{+\infty} e^{\rho_{1}\left(\rho_{\chi}-2 j-(-1)^{j} \rho_{x}\right) z}+\sum_{j=1}^{+\infty} e^{-\rho_{1}\left(\rho_{\chi}+2 j-(-1)^{j} \rho_{x}\right) z} .
\end{aligned}
$$

The ratio test of absolute convergence, for the series $\sum_{j=1}^{+\infty} e^{\rho_{1}\left(\rho_{x}-2 j-(-1)^{j} \rho_{x}\right) z}$, gives

$$
\begin{aligned}
\left|\frac{u_{n+1}}{u_{n}}\right| & =\left|\frac{e^{\rho_{1}\left(\rho_{x}-2(n+1)+(-1)^{n} \rho_{x}\right) z}}{e^{\rho_{1}\left(\rho_{\chi}-2 n-(-1)^{n} \rho_{x}\right) z}}\right| \\
& =\left|e^{2 \rho_{1} z\left\{(-1)^{n} \rho_{x}-1\right\}}\right| .
\end{aligned}
$$


Since $\rho_{x}<1$, and $(-1)^{n} \rho_{x}= \pm \rho_{x}$, it follows that for either sign we have that

$$
\begin{aligned}
& (-1)^{n} \rho_{x}-1=-\rho_{x}-1<0 \\
& (-1)^{n} \rho_{x}-1=+\rho_{x}-1<0 .
\end{aligned}
$$

Then (O.17) gives

$$
L=e^{2 \rho_{1} z\left\{(-1)^{n} \rho_{x}-1\right\}}=e^{-2 \rho_{1} z\left|(-1)^{n} \rho_{x}-1\right|}<1
$$

for all $z$, except for $z=0$ where $L$ equals one. Based on (0.20), and the ratio test of absolute convergence,

$$
\lim _{n \rightarrow \infty}\left|\frac{u_{n+1}}{u_{n}}\right|=L
$$

the series $\sum_{j=1}^{+\infty} e^{\rho_{1}\left(\rho_{\chi}-2 j-(-1)^{j} \rho_{x}\right) z}$ converges absolutely (and therefore converges) for all $z$, except for $z=0$ where the test fails.

Similarly, for the series $\sum_{j=1}^{+\infty} e^{-\rho_{1}\left(\rho_{\chi}+2 j-(-1)^{j} \rho_{x}\right) z}$ we have that

$$
L^{\prime}=\left|\frac{u_{n+1}}{u_{n}}\right|=\left|e^{-2 \rho_{1} z\left\{(-1)^{n} \rho_{x}+1\right\}}\right|,
$$

and based on the relationships

$$
\begin{aligned}
& (-1)^{n} \rho_{x}+1=-\rho_{x}+1>0 \\
& (-1)^{n} \rho_{x}+1=+\rho_{x}+1>0
\end{aligned}
$$

we obtain

$$
L^{\prime}=e^{-2 \rho_{1} z\left\{(-1)^{n} \rho_{x}+1\right\}}=e^{-2 \rho_{1} z\left|(-1)^{n} \rho_{x}+1\right|}<1,
$$

for all $z$, except for $z=0$ where $L^{\prime}$ equals one. Hence, based on the ratio test, the series $\sum_{j=-\infty}^{+\infty} e^{-\rho_{1}\left|\rho_{X}-2 j-(-1)^{j} \rho_{x}\right| z}$ converges for all $z \neq 0$, whereas for $z=0$ we do not know if it is convergant or divergant. 


\section{APPENDIX P}

\section{SOLUTION FOR $\chi_{1} \neq x_{1}$, EQUATION 4.65}

The three-dimensional $G_{F}(\boldsymbol{\chi}, \boldsymbol{x})$ from (4.13) is given as

$$
G_{F}(\boldsymbol{\chi}, \boldsymbol{x})=+\frac{1}{4 \pi} \sum_{j=-\infty}^{+\infty}(-1)^{j} \mathcal{A}_{j}
$$

where

$$
\mathcal{A}_{j}=\frac{1}{\left[\left(\chi_{1}-2 j a-(-1)^{j} x_{1}\right)^{2}+\left(\chi_{2}-x_{2}\right)^{2}+\left(\chi_{3}-x_{3}\right)^{2}\right]^{1 / 2}} .
$$

The first derivative of $\mathcal{A}_{j}$ with respect to $\chi_{1}$ is

$$
\begin{aligned}
\frac{\partial}{\partial \chi_{1}} \mathcal{A}_{j}= & -\left|\chi_{1}-2 j a-(-1)^{j} x_{1}\right| \operatorname{sgn}\left(\chi_{1}-2 j a-(-1)^{j} x_{1}\right) \\
& {\left[\left(\chi_{1}-2 j a-(-1)^{j} x_{1}\right)^{2}+\left(\chi_{2}-x_{2}\right)^{2}+\left(\chi_{3}-x_{3}\right)^{2}\right]^{-3 / 2} }
\end{aligned}
$$

and then the second mixed derivative of $\mathcal{A}_{j}$, using (4.57) and (4.58), is given by

$$
\begin{aligned}
\frac{\partial^{2}}{\partial x_{1} \partial \chi_{1}} \mathcal{A}_{j}= & (-1)^{j}\left[c_{1}^{2}+\left(\chi_{2}-x_{2}\right)^{2}+\left(\chi_{3}-x_{3}\right)^{2}\right]^{-3 / 2} \\
& \times\left[1+2 c_{1} \delta\left(\chi_{1}-2 j a-(-1)^{j} x_{1}\right)\right] \\
- & 3(-1)^{j} c_{1}^{2}\left[c_{1}^{2}+\left(\chi_{2}-x_{2}\right)^{2}+\left(\chi_{3}-x_{3}\right)^{2}\right]^{-5 / 2}
\end{aligned}
$$

where

$$
c_{1}=\left|\chi_{1}-2 j a-(-1)^{j} x_{1}\right|
$$


Therefore we have that

$$
\begin{aligned}
\frac{\partial^{2}}{\partial x_{1} \partial \chi_{1}} G_{F} & =\frac{1}{4 \pi} \sum_{j=-\infty}^{+\infty}\left[c_{1}^{2}+\left(\chi_{2}-x_{2}\right)^{2}+\left(\chi_{3}-x_{3}\right)^{2}\right]^{-3 / 2} \\
& +\frac{1}{4 \pi} \sum_{j=-\infty}^{+\infty} 2 c_{1} \delta\left(\chi_{1}-2 j a-(-1)^{j} x_{1}\right) \times \\
& {\left[c_{1}^{2}+\left(\chi_{2}-x_{2}\right)^{2}+\left(\chi_{3}-x_{3}\right)^{2}\right]^{-3 / 2} } \\
& -\frac{3}{4 \pi} \sum_{j=-\infty}^{+\infty} c_{1}^{2}\left[c_{1}^{2}+\left(\chi_{2}-x_{2}\right)^{2}+\left(\chi_{3}-x_{3}\right)^{2}\right]^{-5 / 2}
\end{aligned}
$$

The series containing the delta function, based on the analysis in page 82 , simplifies to

$$
\begin{aligned}
& \sum_{j=-\infty}^{+\infty} c_{1} \delta\left(\chi_{1}-2 j a-(-1)^{j} x_{1}\right)\left[c_{1}^{2}+\eta^{\prime 2}\right]^{-3 / 2}= \\
& =+\left|\eta_{1}\right| \delta\left(\eta_{1}\right)\left[\eta_{1}^{2}+\eta^{2}\right]^{-3 / 2}
\end{aligned}
$$

where

$$
\eta^{\prime 2}=\left(\chi_{2}-x_{2}\right)^{2}+\left(\chi_{3}-x_{3}\right)^{2}
$$

and $\eta_{1}=\chi_{1}-x_{1}$. Substituting (P.7) into (P.6) we obtain

$$
\begin{aligned}
\frac{\partial^{2}}{\partial x_{1} \partial \chi_{1}} G_{F} & =\frac{1}{4 \pi} \sum_{j=-\infty}^{+\infty}\left[c_{1}^{2}+\eta^{2}\right]^{-3 / 2} \\
& +\frac{1}{4 \pi} 2\left|\eta_{1}\right| \delta\left(\eta_{1}\right)\left[\eta_{1}^{2}+\eta^{2}\right]^{-3 / 2} \\
& -\frac{3}{4 \pi} \sum_{j=-\infty}^{+\infty} c_{1}^{2}\left[c_{1}^{2}+\eta^{2}\right]^{-5 / 2}
\end{aligned}
$$

The two-dimensional FT of (P.9) is given by

$$
F T^{(2)}\left\{\frac{\partial^{2}}{\partial x_{1} \partial \chi_{1}} G_{F}\right\}=\frac{e^{i \boldsymbol{k}^{\prime} \cdot \boldsymbol{x}^{\prime}}}{4 \pi}\left\{I_{3}+\sum_{j=-\infty}^{+\infty} I_{2}\right\},
$$

where

$$
I_{3}=\frac{\left|\eta_{1}\right|}{\pi} \delta\left(\eta_{1}\right) \int_{-\infty}^{+\infty} \int_{-\infty}^{+\infty}\left(\eta_{1}^{2}+\eta^{2}\right)^{-3 / 2} e^{i \boldsymbol{k}^{\prime} \cdot \boldsymbol{\eta}^{\prime}} d \eta_{2} d \eta_{3}
$$


and

$$
\begin{aligned}
I_{2} & =\frac{1}{2 \pi} \int_{-\infty}^{+\infty} \int_{-\infty}^{+\infty}\left(c_{1}^{2}+\eta^{2}\right)^{-3 / 2} e^{i \boldsymbol{k}^{\prime} \cdot \boldsymbol{\eta}^{\prime}} d \eta_{2} d \eta_{3} \\
& -\frac{3}{2 \pi} c_{1}^{2} \int_{-\infty}^{+\infty} \int_{-\infty}^{+\infty}\left(c_{1}^{2}+\eta^{2}\right)^{-5 / 2} e^{i \boldsymbol{k}^{\prime} \cdot \boldsymbol{\eta}^{\prime}} d \eta_{2} d \eta_{3}
\end{aligned}
$$

Here, we have again $\boldsymbol{\eta}^{\prime}=\left(\eta_{2}, \eta_{3}\right)^{T}=\left(\chi_{2}-x_{2}, \chi_{3}-x_{3}\right)^{T}$, and $\boldsymbol{k}^{\prime}=\left(k_{2}, k_{3}\right)^{T}$. In polar coordinates $I_{2}$ is given by

$$
\begin{aligned}
I_{2} & =\frac{1}{2 \pi} \int_{\eta^{\prime}=0}^{+\infty} \int_{\theta=0}^{2 \pi} \eta^{\prime}\left(c_{1}^{2}+\eta^{2}\right)^{-3 / 2} e^{i k^{\prime} \eta^{\prime} \cos \theta} d \eta^{\prime} d \theta \\
& -\frac{3}{2 \pi} c_{1}^{2} \int_{\eta^{\prime}=0}^{+\infty} \int_{\theta=0}^{2 \pi} \eta^{\prime}\left(c_{1}^{2}+\eta^{2}\right)^{-5 / 2} e^{i k^{\prime} \eta^{\prime} \cos \theta} d \eta^{\prime} d \theta,
\end{aligned}
$$

and based on (4.37) it simplifies to

$$
\begin{aligned}
I_{2} & =\int_{\eta^{\prime}=0}^{+\infty} \eta^{\prime}\left(c_{1}^{2}+\eta^{2}\right)^{-3 / 2} J_{0}\left(k^{\prime} \eta^{\prime}\right) d \eta^{\prime} \\
& -3 c_{1}^{2} \int_{\eta^{\prime}=0}^{+\infty} \eta^{\prime}\left(c_{1}^{2}+\eta^{2}\right)^{-5 / 2} J_{0}\left(k^{\prime} \eta^{\prime}\right) d \eta^{\prime}
\end{aligned}
$$

or,

$$
I_{2}=I_{21}-3 c_{1}^{2} I_{22}
$$

Integral $I_{21}$, for $c_{1}=\left|\chi_{1}-2 j a-(-1)^{j} x_{1}\right| \neq 0$, is given by [Erdelyi et al., 1954, p. $7[7]]$

$$
I_{21}=\frac{1}{c_{1} e^{k^{\prime} c_{1}}} .
$$

Condition $c_{1} \neq 0$ is satisfied by all $j \neq 0$; for $j=0$ it applies only for $\chi_{1} \neq x_{1}$. Integral $I_{22}$ is given by [Gradshteyn and Ryzhik, 1980, p. 686[4]]

$$
I_{22}=\int_{\eta^{\prime}=0}^{+\infty} \frac{\eta^{\prime} J_{0}\left(k^{\prime} \eta^{\prime}\right)}{\left(c_{1}^{2}+\eta^{\prime 2}\right)^{\frac{5}{2}}} d \eta^{\prime}=\frac{c_{1}^{-3 / 2} k^{\prime 3 / 2}}{2^{3 / 2} \Gamma\left(\frac{5}{2}\right)} K_{-\frac{3}{2}}\left(k^{\prime} c_{1}\right)
$$

where $K_{-\frac{3}{2}}\left(k^{\prime} c_{1}\right)$, the modified Bessel function. From Abramowitz and Stegun [1972, p. 358, and 375], and Gradshteyn and Ryzhik [1980, p. 967[8.468]] we have that

$$
K_{-\frac{3}{2}}\left(k^{\prime} c_{1}\right)=K_{+\frac{3}{2}}\left(k^{\prime} c_{1}\right)=\frac{\sqrt{\pi}}{\sqrt{2 k^{\prime} c_{1}}} e^{-k^{\prime} c_{1}}\left(1+\frac{1}{k^{\prime} c_{1}}\right),
$$


and then $I_{22}$ becomes

$$
I_{22}=\frac{k^{\prime}}{3 c_{1}^{2}}\left(1+\frac{1}{k^{\prime} c_{1}}\right) e^{-k^{\prime} c_{1}}
$$

Substituting (P.16) and (P.19) in (P.15) we obtain

$$
I_{2}=-\frac{k^{\prime}}{e^{k^{\prime} c_{1}}}
$$

Integral $I_{3}$ is evaluated in polar coordinates as

$$
\begin{aligned}
I_{3} & =2\left|\eta_{1}\right| \delta\left(\eta_{1}\right) \int_{\eta^{\prime}=0}^{+\infty} \eta^{\prime}\left(\eta_{1}^{2}+\eta^{2}\right)^{-3 / 2} J_{0}\left(k^{\prime} \eta^{\prime}\right) d \eta^{\prime} \\
& =2 \delta\left(\eta_{1}\right) e^{-k^{\prime}\left|\eta_{1}\right|}=0 .
\end{aligned}
$$

Here, equation P.16 was used, and therefore the second equality applies for $\eta_{1} \neq 0$ (or $\chi_{1} \neq x_{1}$ ). However, for $\chi_{1} \neq x_{1}$, the delta function, $\delta\left(\eta_{1}\right)=\delta\left(\chi_{1}-x_{1}\right)=0$, and hence $I_{3}$ equals zero. Substituting (P.20) and (P.21) in (P.10) we finally obtain

$$
F T^{(2)}\left\{\frac{\partial^{2}}{\partial x_{1} \partial \chi_{1}} G_{F}\right\}=-\frac{k^{\prime}}{4 \pi} e^{i \boldsymbol{k}^{\prime} \cdot \boldsymbol{x}^{\prime}} \sum_{j=-\infty}^{+\infty} e^{-k^{\prime}\left|\chi_{1}-2 j a-(-1)^{j} x_{1}\right|}
$$

valid only for $\chi_{1} \neq x_{1}$. 


\section{APPENDIX Q}

\section{PROGRAM PLATES.FOR}

program plates

implicit real*8 $(\mathrm{a}-\mathrm{h}, \mathrm{o}-\mathrm{z})$

$c$ ux Gauss points in the direction of $u$

$c$ uw Gauss weights in the direction of $u$

dimension $\mathrm{ux}(2000), \mathrm{xx}(2000), \mathrm{uw}(1000), \mathrm{xw}(1000)$

dimension nptsu(101), nptsx(101), xx1(101), xx2(101), xu1(101)

dimension $x u 2(101), x 1000(1000), w 1000(1000), x 360(360), w 360(360)$

dimension domain(200), surface(400)

common /one/rho, aksi

c Read in data

$\operatorname{open}\left(10, \mathrm{file}=' \mathrm{~g} 1000^{\prime}\right)$

do $i=1,1000$

$$
\operatorname{read}(10, *) \times 1000(i)
$$

end do

do $i=1,1000$

$$
\operatorname{read}(10, *) \mathrm{w} 1000(\mathrm{i})
$$


end do

close (10)

open $(10, \mathrm{file}=$ 'g360')

do $i=1,360$

$\operatorname{read}(10, *) \times 360$ (i)

end do

do $i=1,360$

$\operatorname{read}(10, *)$ w360 (i)

end do

close (10)

do $i=1,101$

$\operatorname{domain}(i)=0.0$

$\operatorname{surface}(i)=0.0$

end do

do $i=102,202$

$\operatorname{surface}(i)=0.0$

end do

open(10,file='input.dat')

$\operatorname{read}(10, *)$ rho,aksi,nintervals

do $n n=1$, nintervals

print*,nn 


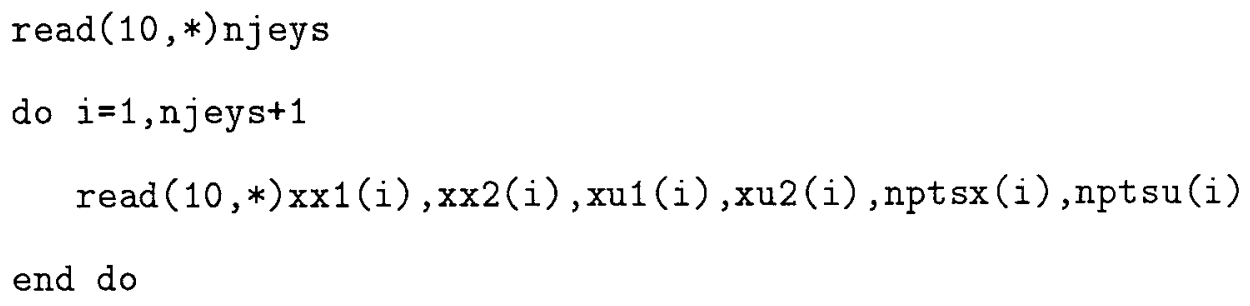

c For every $j$ calculate the appropriate gauss points

$c$ and Gauss weights

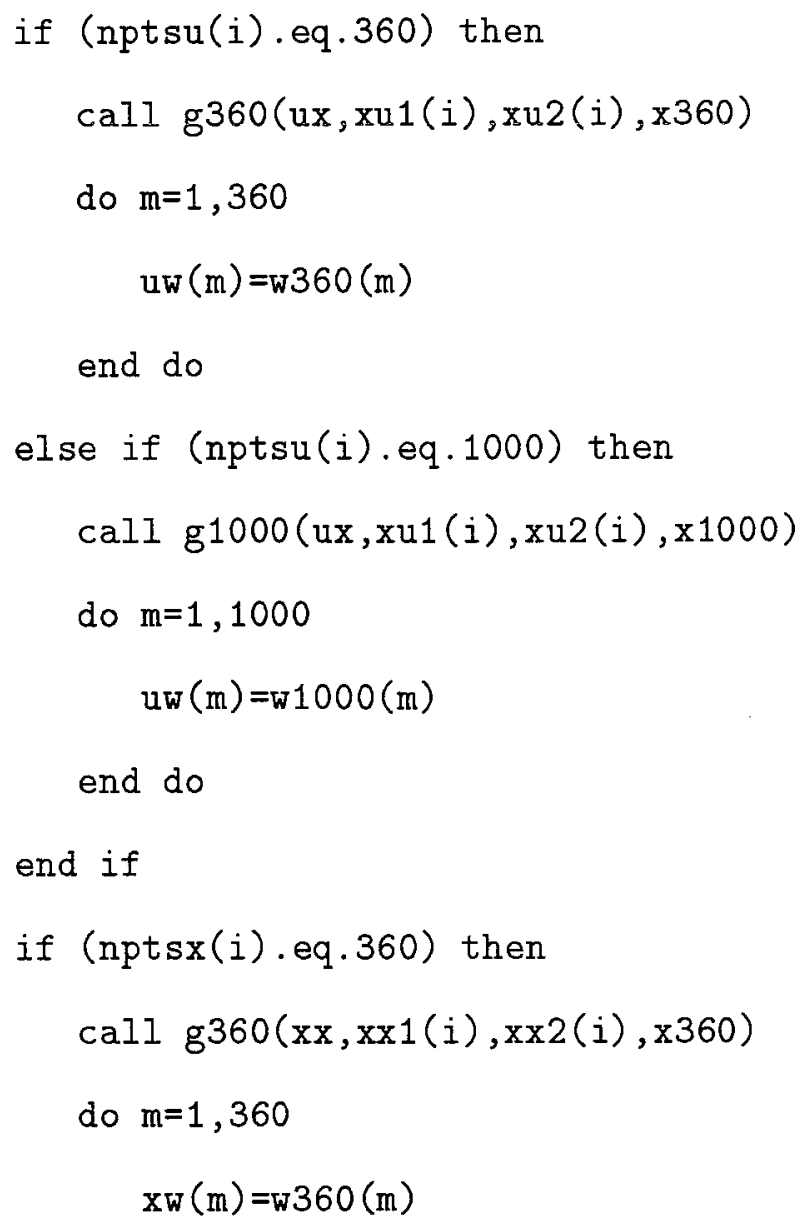


end do

else if (nptsx(i).eq.1000) then

call $\mathrm{g} 1000(\mathrm{xx}, \mathrm{xx} 1(\mathrm{i}), \mathrm{xx2}(\mathrm{i}), \mathrm{x} 1000)$

do $m=1,1000$

$\mathrm{xw}(\mathrm{m})=\mathrm{w} 1000(\mathrm{~m})$

end do

end if

$\operatorname{sum} 2=0.0$

$\operatorname{sum} 3=0.0$

c Domain integral

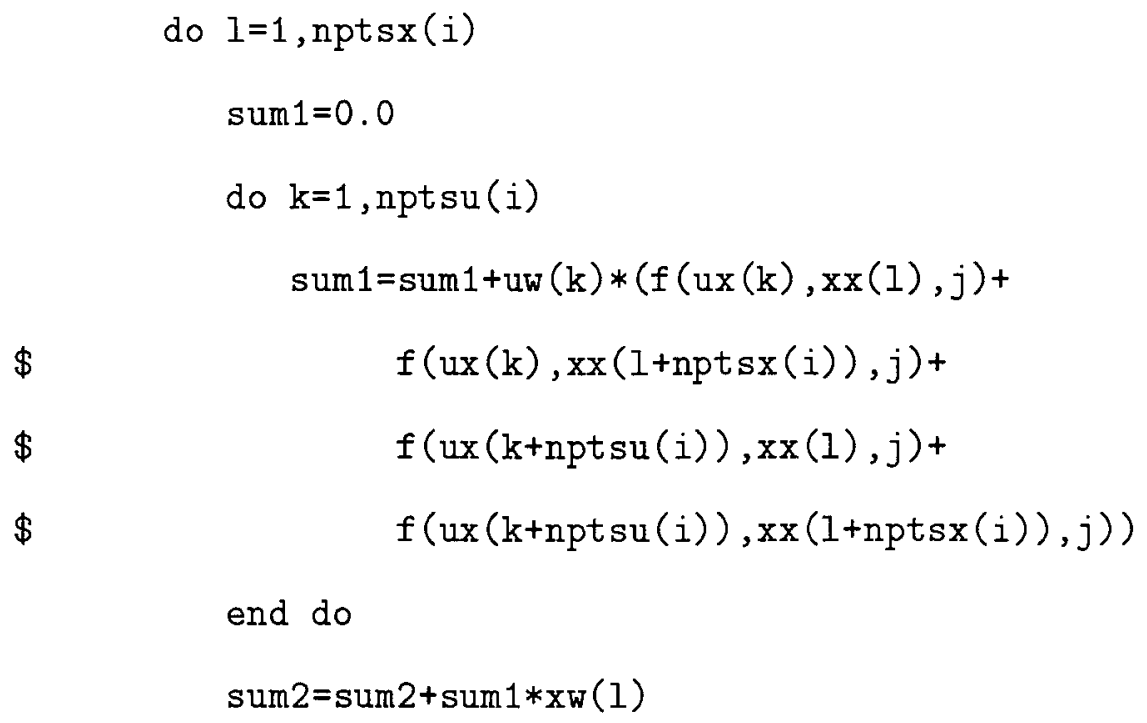




$$
\operatorname{domain}(i)=\operatorname{domain}(i)+\operatorname{sum} 2 *(\operatorname{xx} 2(i)-\operatorname{xx} 1(i)) *
$$$$
(\operatorname{xu} 2(i)-\operatorname{xu} 1(i)) / 4.0
$$

else

$$
\operatorname{domain}(i)=\operatorname{domain}(i)+\operatorname{sum} 2 *(x \times 2(i)-x \times 1(i)) *(x u 2(i)-
$$

$\$$

$$
\operatorname{xu} 1(i)) / 2.0
$$

end if

$\operatorname{surface}(i)=\operatorname{surface}(i)+\operatorname{sum} 3 *(x \times 2(i)-x \times 1(i)) / 2.0$

if $(j . n e .0)$ then

c Surface integral for $-j$

$\operatorname{sum} 3=0.0$

do $1=1, \operatorname{nptsx}(i)$

sum3 $=\operatorname{sum} 3+x w(1) *(g(x x(1),-j)+g(x x(1+n p t s x(i)),-j))$

end do

$\operatorname{surface}(101+j)=\operatorname{surface}(101+j)+\operatorname{sum} 3 *(\operatorname{xx} 2(i)-x \times 1(i)) / 2.0$

end if

end do

end do

close (10)

njeys $=100$

open $(10$, file='results' $)$

write(10,'(') Rho=' ',f6.3,' Aksi=' ',f5.3)')rho, aksi

do $i=1$, njeys +1 


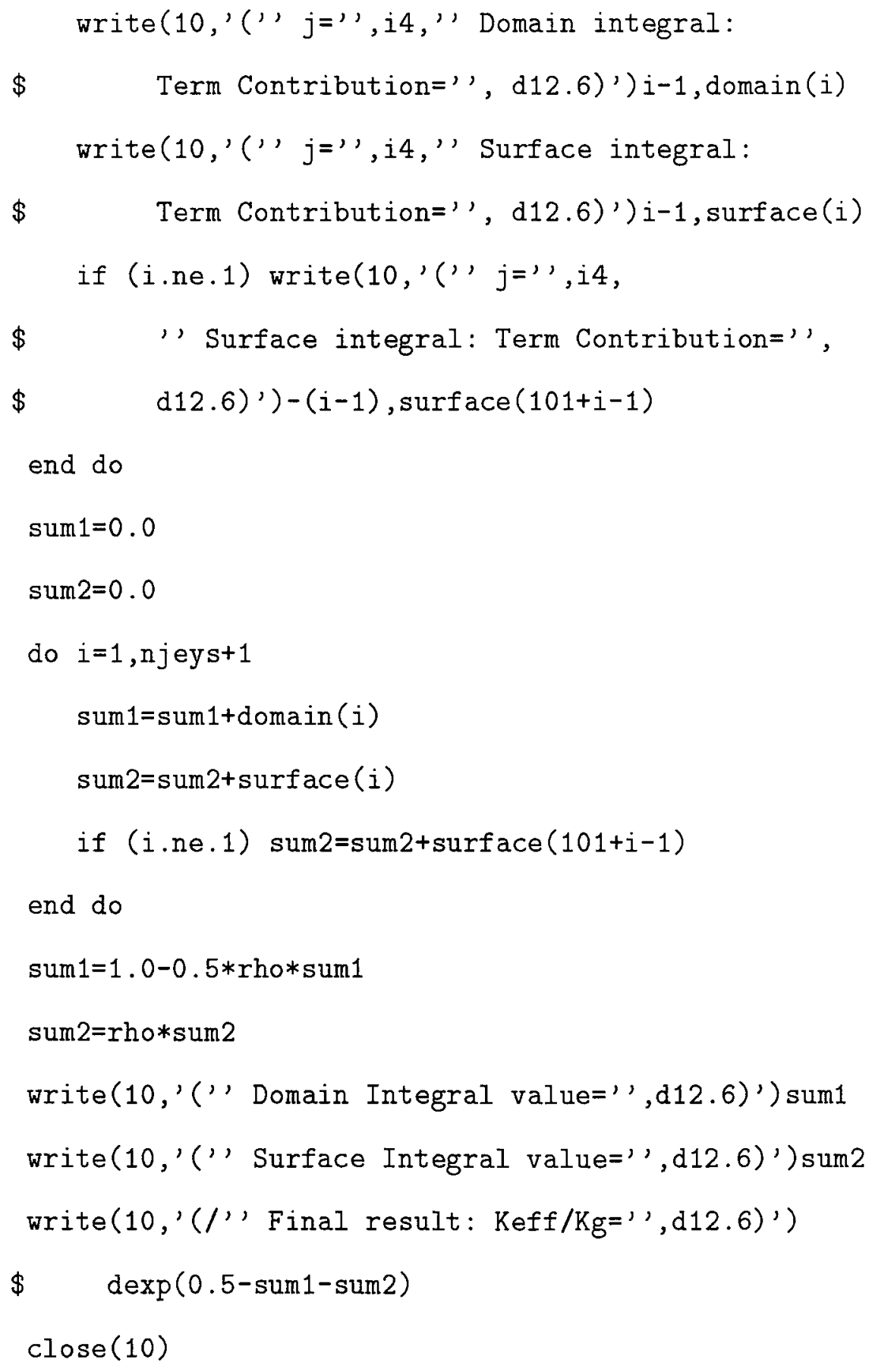


end

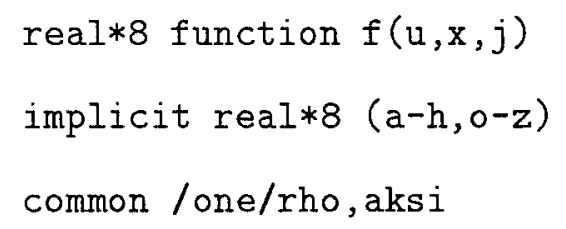


end

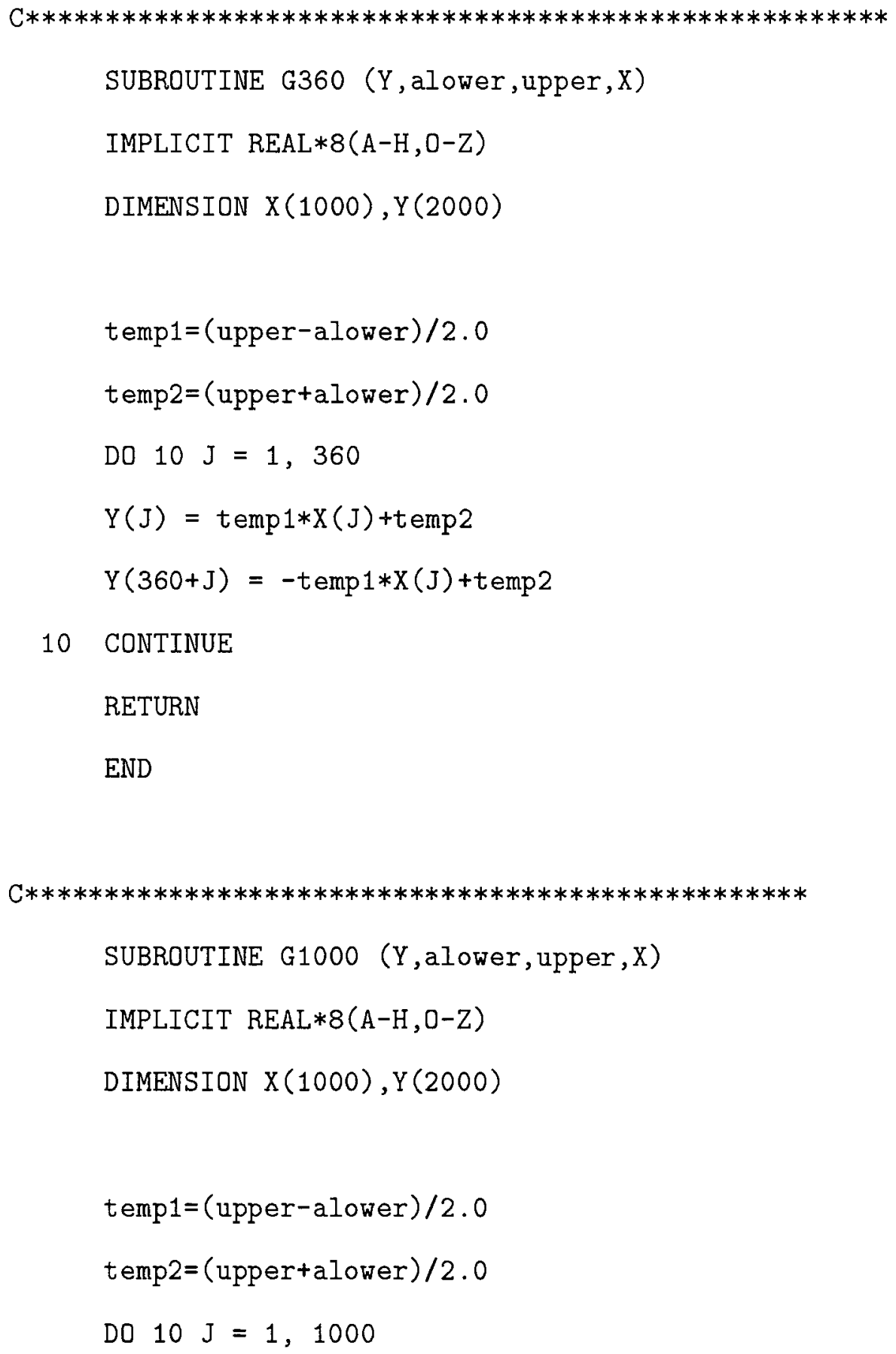




$$
\begin{aligned}
& Y(J)=\operatorname{temp} 1 * X(J)+t \operatorname{emp} 2 \\
& Y(1000+J)=-t \operatorname{emp} 1 * X(J)+t e m p 2
\end{aligned}
$$

\section{CONTINUE}

\section{RETURN}

END

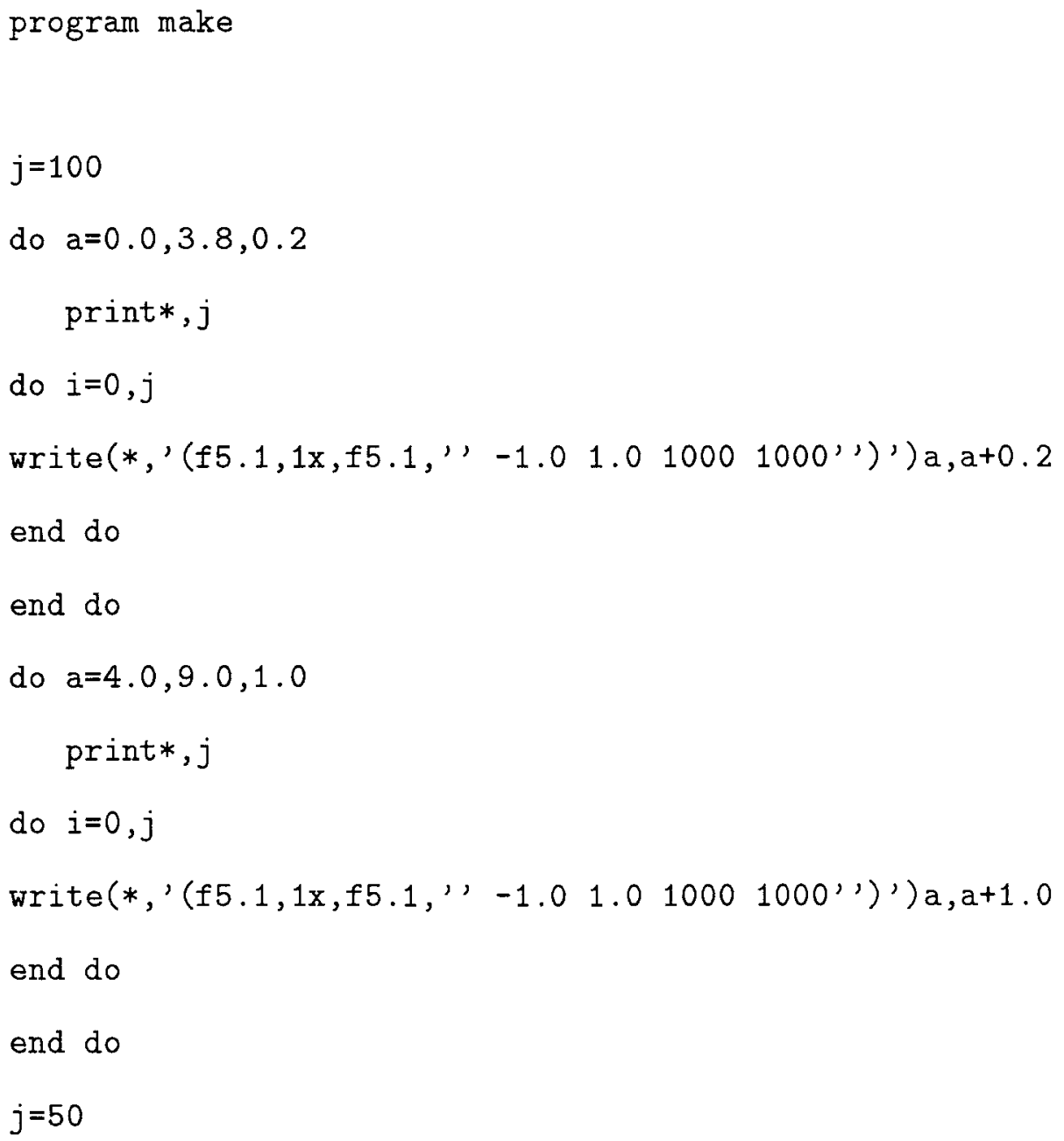




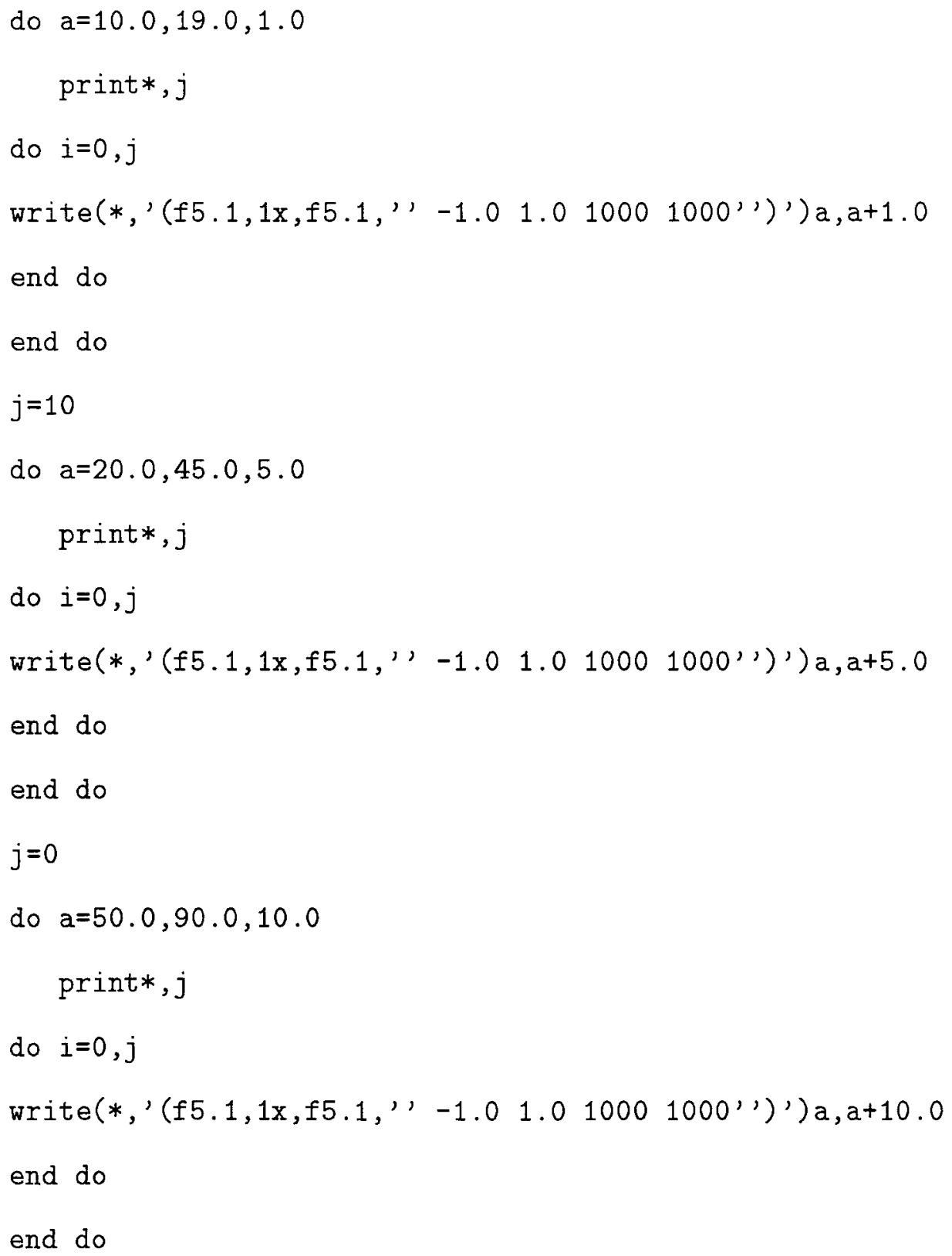


write(*,'(f6.1,1x,f6.1,', -1.01 .01000 1000'')') a, a+100.0

end do

end do

print*, $j$

do $i=0, j$

print*, ' $800.03000 .0-1.01 .010001000$ '

end do

stop

end 


\section{LIST OF REFERENCES}

Ababou, R., Three-dimensional flow in random porous media, Ph.D. dissertation, Ralph Parsons Lab., Mass. Inst. of Technol., Cambridge, 1988.

Ababou, R., and E. F. Wood, Comment on "Effective groundwater model parameter values: Influence of spatial variability of hydraulic conductivity, leakance, and recharge" by J.J. Gómez-Hernández and S.M. Gorelick, Water Resour. Res., 26(8), 1843-1846, 1990a.

Ababou, R., and E. F. Wood, Correction to "Comment on 'Effective groundwater model parameter values: Influence of spatial variability of hydraulic conductivity, leakance, and recharge' by J.J. Gómez-Hernández and S.M. Gorelick", Water Resour. Res., 26(12), 2945, 1990 b.

Ababou, R., and L. W. Gelhar, Self-similar randomness and spectral conditioning: Analysis of scale effects in subsurface hydrology, Dynamics of fluids in hierarchical porous media, Academic Press, 393-428, 1990.

Ababou, R., Identification of effective conductivity tensor in randomly heterogeneous and stratified aquifers, Proceedings of the 5th Canadian/American conference on hydrogeology: Parameter identification and estimation for aquifer and reservoir characterization: National Well Water Association, Dublin, Ohio, p. $155-157,1991$.

Abramowitz, M., and I. A. Stegun , Handbook of Mathematical Functions, Dover Pub., New York, 1972.

Bakr, A. A., Effect of spatial variations of hydraulic conductivity on groundwater flow, Ph.D. dissertation, N. M. Inst. of Min. and Technol., Socorro, 1976. 
Bakr, A. A., L. W. Gelhar, A. L. Gutjahr, and J. R. MacMillan, Stochastic analysis of spatial variability in subsurface flows, 1, Comparison of one- and threedimensional flows, Water Resour. Res., 14(2), 263-271, 1978.

Baveye, P., and G. Sposito, The operational significance of the continuum hypothesis in the theory of water movement through soils and aquifers, Water Resour. Res., 20(5), 521-530, 1984.

Baveye, P., and G. Sposito, Macroscopic balance equations in soils and aquifers: The case of space- and time-dependent instrumental response, Water Resour. Res., 21(8), 1116-1120, 1985.

Bear, J., Dynamics of Fluids in Porous Media, American Elsevier, New York, 1972.

Beran, M. J., Statistical Continuum Theories, Interscience, New York, 1968.

Bleistein, N., and R. A. Handelsman, Asymptotic Expansions of Integrals, Dover Pub., New York, 1986.

Clifton, P. M., Statistical inverse modeling and geostatistical analysis of the Avra Valley aquifer, M.S. thesis, Dep. Hydrol. and Water Resour., Univ. of Ariz., Tucson, 1981.

Clifton, P. M., and S. P. Neuman, Effects of Kriging and inverse modeling on conditional simulation of the Avra Valley aquifer in Southern Arizona, Water Resour. Res., 18(4), 1215-1234, 1982.

Cushman, J. H., Comment on " Three-dimensional stochastic analysis of macrodispersion in aquifers", by L. W. Gelhar and C. L. Axness, Water Resour. Res., 19(6), 1641-1642, 1983a. 
Cushman, J. H., Volume averaging, probabilistic averaging, and ergodicity, $A d v$. Water Resour., 6, 182-184, 1983b.

Cushman, J. H., On unifying the concepts of scale, instrumentation, and stochastics in the development of multiphase transport theory, Water Resour. Res., 20(11), $1668-1676,1984$.

Cushman, J. H., On measurement, scale, and scaling, Water Resour. Res., 22(2), $129-134,1986$.

Cushman, J. H., Development of stochastic partial differential equations for subsurface hydrology, Jour. Stoch. Hydr. $\mathscr{G}$ Hydraul., 1(4), 241-262, 1987.

Cushman, J. H., On the inseparability of the measurement process from theories of transport in heterogeneous materials, in Concise Encyclopedia of Environmental Systems, edited by P. Young, Pergamon, New York, in press, 1993.

Dagan, G., Models of groundwater flow in statistically homogeneous porous formations, Water Resour. Res., 15(1), 47-63, 1979.

Dagan, G., Analysis of flow through heterogeneous random aquifers by the method of embedding matrix, 1, Steady flow, Water Resour. Res., 17(1), 107-121, 1981.

Dagan, G., Stochastic modeling of groundwater flow by unconditional and conditional probabilities, 1, Conditional simulation and the direct problem, Water Resour. Res., 18(4), 813-833, $1982 \mathrm{a}$.

Dagan, G., Analysis of flow through heterogeneous random aquifers, 2, Unsteady flow in confined formations, Water Resour. Res., 18(5), 1571-1585, $1982 \mathrm{~b}$.

Dagan, G., A note on higher-order corrections of the head covariances in steady aquifer flow, Water Resour. Res., 21(4), 573-578, 1985. 
Dagan, G., Statistical theory of groundwater flow and transport: Pore to laboratory, laboratory to formation, and formation to regional scale, Water Resour. Res., 22(9), 120S-134S, 1986.

Dagan, G., Flow and Transport in Porous Formations, 465 pp., Springer-Verlag, New York, 1989.

Delhomme, J. P., Spatial variability and uncertainty in groundwater flow parameters: A geostatistical approach, Water Resour. Res., 15(2), 269-280, 1979.

Desbarats, A. J., Numerical estimation of effective permeability in sand-shale formations, Water Resour. Res., 23(2), 273-286, 1987 a.

Desbarats, A. J., Stochastic modeling of flow in sand-shale sequences, Ph.D. dissertation, Dep. Appl. Earth Sci., Stanford University, Stanford, 1987b.

Desbarats, A. J., and R. Dimitrakopoulos, Geostatistical modeling of transmissibility for 2D reservoir studies, SPE Form. Eval., 437-443, 1990.

Desbarats, A. J., Spatial averaging of hydraulic conductivity in three-dimensional heterogeneous porous media, Math. Geol., 24(3), 249-267, 1992 a.

Desbarats, A. J., Spatial averaging of transmissivity in heterogeneous fields with flow toward a well, Water Resour. Res., 28(3), 757-767, $1992 \mathrm{~b}$.

Desbarats, A. J., personal communication, July 1993.

Deutsch, C., Calculating effective absolute permeability in sandstone/shale sequences, SPE Form. Eval., 343-348, 1989.

Dykaar, B. B., and P. K. Kitanidis, Determination of the effective hydraulic conductivity for heterogeneous porous media using a numerical spectral approach, 1, Method, Water Resour. Res., 28(4), 1155-1166, 1992a. 
Dykaar, B. B., and P. K. Kitanidis, Determination of the effective hydraulic conductivity for heterogeneous porous media using a numerical spectral approach, 2, Results, Water Resour. Res., 28(4), 1167-1178, 1992b.

El-Kadi, A. I., and W. Brutsaert, Applicability of effective parameters for unsteady flow in nonuniform aquifers, Water Resour. Res., 21(2), 183-198, 1985.

Erdelyi, A., W. Magnus, F. Oberhettinger and F. Tricomi, Tables of Integral Transforms, Vol. 2, McGraw-Hill, 1954.

Fenton, G. A., and D. V. Griffiths, Statistics of block conductivity through a simple bounded stochastic medium, Water Resour. Res., 29(6), 1825-1830, 1993.

Freeze, R. A., A stochastic-conceptual analysis of one-dimensional groundwater flow in nonuniform homogeneous media, Water Resour. Res., 11(5), 725-741, 1975.

Gaughan, E. D., Introduction to Analysis, 226 pp., Brooks/Cole Pub. Co., Monterey, California, 1987.

Gelhar, L. W., and C. L. Axness, Stochastic analysis of macrodispersion in threedimensionally heterogeneous aquifers, Report No. H-8, N. M. Inst. of Min. and Technol., Hydrology Research Program, Socorro, 1981.

Gelhar, L. W., and C. L. Axness, Three-dimensional stochastic analysis of macrodispersion in aquifers, Water Resour. Res., 19(1), 161-180, 1983a.

Gelhar, L. W., and C. L. Axness, Reply to comments by J.H. Cushman (ibid) Water Resour. Res., 19(6), 1643-1644, 1983b.

Gelhar, L. W., Stochastic subsurface hydrology. From theory to applications, Water Resour. Res., 22(9), 135S-145S, 1986. 
Gómez-Hernández, J. J., and S. M. Gorelick, Effective groundwater model parameter values: Influence of spatial variability of hydraulic conductivity, leakance, and recharge, Water Resour. Res., 25(3), 405-419, 1989.

Gómez-Hernández, J. J., and S. M. Gorelick, Reply, Water Resour. Res., 26(8), $1847-1848,1990$.

Gómez-Hernández, J. J., A stochastic approach to the simulation of block conductivity fields conditioned upon data measured at a smaller scale, Ph.D. dissertation, Dep. Appl. Earth Sci., Stanford University, Stanford, 1991.

Gradshteyn, I. S., and I. M. Ryzhik, Table of Integrals, Series, and Products, Academic Press, 1980.

Greenberg, M. D., Application of Green's Functions in Science and Engineering, Prentice-Hall, New Jersey, 1971.

Greenberg, M. D., Foundations of Applied Mathematics, Prentice-Hall, New Jersey, 1978.

Gutjahr, A. L., L. W. Gelhar, A. A. Bakr, and J. R. MacMillan, Stochastic analysis of spatial variability in subsurface flows, 2, Evaluation and application, Water Resour. Res., 14(5), 953-959, 1978.

Gutjahr, A. L., and L. W. Gelhar, Stochastic models of subsurface flow: Infinite versus finite domains and stationarity, Water Resour. Res., 17(2), 337-350, 1981.

Gutjahr, A. L., Stochastic models of subsurface flow: Log linearized Gaussian models are "exact" for covariances, Water Resour. Res., 20(12), 1909-1912, 1984. 
Haberman, R., Elementary Applied Partial Differential Equations, Prentice-Hall, New Jersey, 1987.

Hoeksema, R. J., and P. K. Kitanidis, Analysis of the spatial structure of properties of selected aquifers, Water Resour. Res., 21(4), 563-572, 1985.

Holmes, D., M. Abbott, and M. Brightman, Site characterization and validation single borehole hydraulic testing of " $\mathrm{C}$ ' boreholes, simulated drift experiment and small scale hydraulic testing, stage 3, Stripa Project 90-10, Technical Report, April 1990.

IMSL, International Mathematical and Statistical Library, Edition 2.1, Houston, Texas, IMSL Ltd., 1989.

Indelman, P., and G. Dagan, Upscaling of permeability of anisotropic heterogeneous formations, 1, The general framework, Water Resour. Res., 29(4), 917-923, 1993a.

Indelman, P., and G. Dagan, Upscaling of permeability of anisotropic heterogeneous formations, 2, General structure and small perturbation analysis, Water Resour. Res., 29(4), 925-933, 1993 b.

Indelman, P., and G. Dagan, Upscaling of permeability of anisotropic heterogeneous formations, 3, Applications, Water Resour. Res., 29(4), 935-943, 1993c.

Isaaks, E. H., and R. M. Srivastava, An Introduction to Applied Geostatistics, Oxford University Press, 1989.

Jones, J. W., E. S. Simpson, S. P. Neuman, and W. S. Keys, Field and theoretical investigations of fractured crystalline rock near Oracle, Arizona, NUREG-CR3736, prepared for U.S. N.R.C., 1985. 
Journel, A. G., Geostatistics for conditional simulation of ore bodies, Econ. Geol, $69(5), 673-687,1974$.

Journel, A. G., and Ch. J. Huijbregts, Mining Geostatistics, Academic Press, London, 1978.

Journel, A. G., C. Deutsch, and A. J. Desbarats, Power averaging for block effective permeability, SPE 15128, presented at the 56th California Regional Meeting of the SPE, Oakland, California, April 2-4, 1986.

Kitanidis, P. K., Effective hydraulic conductivity for gradually varying flow, Water Resour. Res., 26(6), 1197-1208, 1990.

Kitanidis, P. K., personal communication, 1994.

Knopp, K., Theory and Applications of Infinite Series, Hafner Pub. Co., 1947.

Landau, L. D., and E. M. Lifshitz, Electrodynamics of Continuous Media, Pergamon, New York, 1960.

Levin, O., Dep. Hydrol. and Water Resour., Univ. of Ariz., Tucson, personal communication, 1992.

Macsyma, Macsyma Inc., 20 Academy Street, Arlington, MA 02174, 1992.

Maple, Waterloo Maple Software, 160 Columbia Street West, Waterloo, Ontario, Canada N2L 3L3, 1992.

de Marsily, G., Quantitative Hydrogeology, Academic Press, London, 1986.

Matheron, G., Éléments pour une Théorie des Milieux Poreux, 166 pp., Masson et Cie, Paris, 1967. 
Matheron, G., The Theory of Regionalized Variables and its Applications, Les cahiers du contre de morphologie mathematique de Fontainebleau, No.5, 1971.

Mood, A. M., and F. A. Graybill, Introduction to the Theory of Statistics, 443 pp., McGraw-Hill, New York, 1963.

Naff, R. L., and A. V. Vecchia, Stochastic analysis of three-dimensional flow in a bounded domain, Water Resour. Res., 22(5), 695-704, 1986.

Naff, R. L., Radial flow in heterogeneous porous media: An analysis of specific discharge, Water Resour. Res., 27(3), 307-316, 1991.

Neuman, S. P., Winter, C. L., and C. M. Newman, Stochastic theory of field-scale Fickian dispersion in anisotropic porous media, Water Resour. Res., 23(3), 453-466, 1987.

Neuman, S. P., and J. S. Depner, Use of variable-scale pressure test data to estimate the log hydraulic conductivity covariance and dispersivity of fractured granites near Oracle, Arizona, J. Hydrol., 102(1-4), 475-501, 1988.

Neuman, S. P., Universal scaling of hydraulic conductivities and dispersivities in geologic media, Water Resour. Res., 26(8), 1749-1758, 1990.

Neuman, S. P., Reply to Comment by M.P. Anderson, Water Resour. Res., 27(6), $1381-1382,1991$.

Neuman, S. P., Orr, S., Levin, O., and E. Paleologos, Theory and high-resolution finite element analysis of $2-\mathrm{D}$ and $3-\mathrm{D}$ effective permeabilities in strongly heterogeneous porous media, in Mathematical Modeling in Water Resources, vol. 2, edited by T. F. Russell, R. E. Ewing, C. A. Brebbia, W. G. Gray, and G. F. Pinder, pp. 118-136, Elsevier Applied Science, New York, 1992. 
Neuman, S. P., Lecture notes for independent study HWR 699, Dep. Hydrol. and Water Resour., Univ. of Ariz., Tucson, 1992.

Neuman, S. P., and S. Orr, Prediction of steady state flow in nonuniform geologic media by conditional moments: exact nonlocal formalism, effective conductivities, and weak approximation, Water Resour. Res., 29(2), 341-364, 1993.

Neuman, S. P., Levin, O., Orr, S., Paleologos, E., Zhang, D., and Y.-K. Zhang, Nonlocal representations of subsurface flow and transport by conditional moments, Computational Stochastic Mechanics, edited by: A. H.-D. Cheng, and C. Y. Yang, Computational Mechanics Publications, Billerica, Massachussets, 1993.

Neuman, S. P., Generalized scaling of permeabilities: Validation and effect of support scale, Geophys. Res. Let., 21(5), 349-352, 1994.

Noetinger, B., The effective permeability of a heterogeneous porous medium, Institut Francais du Petrole, Division Gisements, Project: B 4332044, 1990.

Orr, S., Stochastic approach to steady state flow in nonuniform geologic media, Ph.D. Dissertation, Dep. Hydrol. and Water Resour., Univ. of Ariz., Tucson, 1993.

Papoulis, A., Probability, Random Variables, and Stochastic Processes, McGrawHill, New York, 1984.

Poley, A.D., Effective permeability and dispersion in locally heterogeneous aquifers, Water Resour. Res., 24(11), 1921-1926, 1988.

Priestley, M.B., Spectral Analysis and Time Series, Academic Press, 1989.

Ray, W. O., Real Analysis, Prentice Hall, 1988. 
Rubin, Y. and G. Dagan, Stochastic analysis of boundaries effects on head spatial variability in heterogeneous aquifers, 1, Constant head boundary, Water Resour. Res., 24(10), 1689-1697, 1988.

Rubin, Y., and G. Dagan, Stochastic analysis of boundaries effects on head spatial variability in heterogeneous aquifers, 2, Impervious boundary, Water Resour. Res., 25(4), 707-712, 1989.

Rubin, Y., and J. J. Gómez-Hernández, A stochastic approach to the problem of upscaling of conductivity in disordered media: Theory and unconditional numerical simulations, Water Resour. Res., 26(4), 691-701, 1990.

Sanchez-Villa, X., and J. Carrera, E.T.S.I. Caminos, Universidad Politécnica de Cataluña, Barcelona, Spain, personal communication, 1993.

Shvidler, M. I., Filtration flows in heterogeneous media (in Russian), Izv. Akad. Nauk SSSR Mech., 3, 185-190, 1962.

Smith, J. L., Spatial variability of flow parameters in a stratified sand, Math. Geol., 13(1), 1-21, 1981.

Smith, L., and R. A. Freeze, Stochastic analysis of steady state groundwater flow in a bounded domain, 1, One- dimensional simulations, Water Resour. Res., 15(3), 521-528, 1979a.

Smith, L., and R. A. Freeze, Stochastic analysis of steady state groundwater flow in a bounded domain, 2, Two- dimensional simulations, Water Resour. Res., $15(6), 1543-1559,1979 \mathrm{~b}$.

Spiegel, M. R., Complex Variables, Schaum's Outline Series, 1964. 
Stakgold, I., Green's Functions and Boundary Value Problems, John Wiley \& Sons, New York, 1979.

Sudicky, E. A., A natural gradient experiment on solute transport in a sand aquifer: Spatial variability of hydraulic conductivity and its role in the dispersion process, Water Resour. Res., 22(13), 2069-2082, 1986.

Tompson, A. F. B., R. Ababou, and L. W. Gelhar, Implementation of the turning bands random field generator, Water Resour. Res., 25(10), 2227-2243, 1989.

Zauderer, E., Partial Differential Equations of Applied Mathematics, John Wiley \& Sons, New York, 1983, pp. 779.

Zhang, Y. K., A Quasilinear Theory of Time-Dependent Nonlocal Dispersion in Geologic Media, Ph.D. dissertation, Dep. Hydrol. and Water Resour., Univ. of Ariz., Tucson, 1990.

Winberg, A., Analysis of spatial correlation of hydraulic conductivity data from the Stripa mine, Stripa Project 91-28, Technical Report, November 1991.

Woodbury, A. D., and E. A. Sudicky, The geostatistical characteristics of the Borden aquifer, Water Resour. Res., 27(4), 533-546, 1991. 